\title{
A Measurement of the B ---> Eta/C K Branching Fraction Using the BaBar Detector
}

\author{
Frank Jackson \\ Stanford Linear Accelerator Center \\ Stanford University \\ Stanford, CA 94309
}

SLAC-Report-804

Prepared for the Department of Energy

under contract number DE-AC02-76SF00515

Printed in the United States of America. Available from the National Technical Information Service, U.S. Department of Commerce, 5285 Port Royal Road, Springfield, VA 22161. 
This document, and the material and data contained therein, was developed under sponsorship of the United States Government. Neither the United States nor the Department of Energy, nor the Leland Stanford Junior University, nor their employees, nor their respective contractors, subcontractors, or their employees, makes an warranty, express or implied, or assumes any liability of responsibility for accuracy, completeness or usefulness of any information, apparatus, product or process disclosed, or represents that its use will not infringe privately owned rights. Mention of any product, its manufacturer, or suppliers shall not, nor is it intended to, imply approval, disapproval, or fitness of any particular use. A royalty-free, nonexclusive right to use and disseminate same of whatsoever, is expressly reserved to the United States and the University. 


\title{
A Measurement of the $B \rightarrow \eta_{c} K$ Branching Fraction Using the $B A B A R$ Detector
}

\author{
Frank Jackson
}

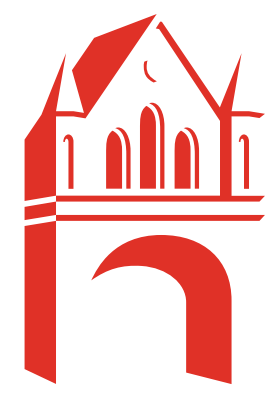

THE UNIVERSITY

of MANCHESTER

\author{
Particle Physics Group \\ Department of Physics and Astronomy \\ May, 2003
}

A thesis submitted to The University of Manchester for the degree of

Doctor of Philosophy in the Faculty of Science and Engineering 


\section{Contents}

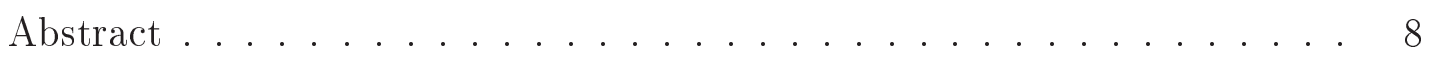

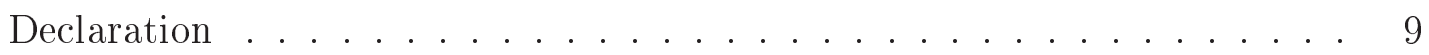

The Author .......................... 10

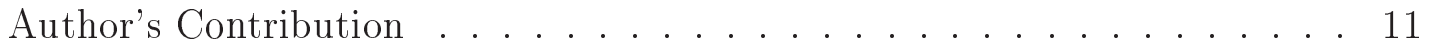

Acknowledgements ......................... 12

1 Theory 14

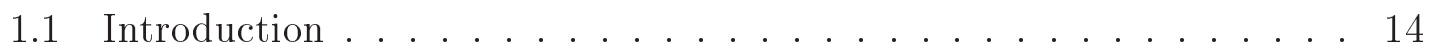

$1.2 C P$ Violation . . . . . . . . . . . . . . . 15

1.3 Introduction to the $B$ Meson System . . . . . . . . . . 15

$1.4 C P$ Violation in the $B$ Meson System . . . . . . . . . . . . 18

1.4.1 Comparison with the Neutral Kaon System . . . . . . . . . . . 18

1.4.2 Types of $C P$ Violation in the $B$ Meson System . . . . . . . . . 19

1.4.3 Measuring $C P$ Violation in $B$ Meson Decays . . . . . . . . . . 21

1.5 The CKM Matrix and the Unitarity Triangle . . . . . . . . . . . 22

1.5.1 Theoretical Background ............... 22

1.5.2 Experimental Measurements ............. 24 
1.6 The Decays $B \rightarrow \eta_{c} K \ldots \ldots \ldots \ldots \ldots$

1.6.1 Predictions of $B \rightarrow \eta_{c} K$ Branching Fractions . . . . . . . . 27

1.6.2 The $\eta_{c}$ Meson . . . . . . . . . . . . . . . 30

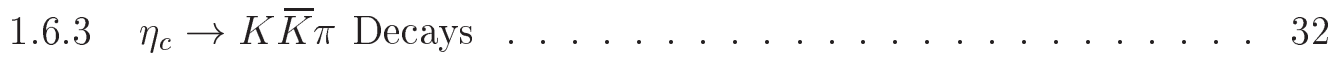

2 The $B A B A R$ Experiment $\quad 36$

2.1 The PEP-II Storage Rings and Interaction Point . . . . . . . . . 37

2.2 The BABAR Detector Overview . . . . . . . . . . . . . 39

2.3 The Silicon Vertex Tracker . . . . . . . . . . . . . . 41

2.4 The Drift Chamber . . . . . . . . . . . . . . . 45

2.5 The Detector of Internally Reflected Cherenkov Light . . . . . . . . . 50

2.6 The Electromagnetic Calorimeter . . . . . . . . . . . 52

2.7 The Superconducting Coil . . . . . . . . . . . . 56

2.8 The Instrumented Flux Return _. . . . . . . . . . . . 57

2.9 The Trigger ......................... 59

2.9.1 The Level 1 Trigger . . . . . . . . . . . . . . 59

2.9.2 The Level 3 trigger . . . . . . . . . . . . 60

2.10 Data Acquisition and Event Reconstruction . . . . . . . . . 61

2.10.1 Data Acquisition ................. 61

2.10.2 Online Reconstruction . . . . . . . . . . . . 62

2.10 .3 Reprocessing ................... 64

3 Calibration of the Electromagnetic Calorimeter with $\pi^{0} \rightarrow \gamma \gamma$ Events 65 
3.1 EMC Calibration . . . . . . . . . . . . . . . 66

3.1 .1 Electronics Calibration . . . . . . . . . . . . . . 66

3.1.2 Single Crystal Calibration . . . . . . . . . . . 66

3.1 .3 Cluster Calibration . . . . . . . . . . . . . . . . 67

3.2 Overview of $\pi^{0}$ Calibration Method . . . . . . . . . . . . 69

3.2.1 Parametrization of Photon Energy Correction . . . . . . . 69

3.2.2 Calibration Determination from $\pi^{0}$ Mass Constraint . . . . . 70

$3.3 \quad \pi^{0}$ Peak Position Determination . . . . . . . . . . . . . 71

3.3 .1 Overview ..................... 71

3.3.2 $\pi^{0}$ Selection and Histogram Formation $\ldots \ldots \ldots \ldots$

3.3.3 Background-Subtracted Mean Calculation . . . . . . . . 74

3.3.4 Peak Calculation from Mean . . . . . . . . . . . . 77

3.4 Calibration Coefficient Extraction . . . . . . . . . . 78

3.4 .1 Energy Binning . . . . . . . . . . . . . . . 78

3.4.2 Calculation of Calibration Coefficients . . . . . . . 81

3.5 Calibration Performance . . . . . . . . . . . . . 85

3.6 Statistical and Systematic Errors . . . . . . . . . . . . . 87

3.6 .1 Statistical Errors $\ldots \ldots \ldots \ldots \ldots$. . . . . . 87

3.6 .2 Systematic Errors . . . . . . . . . . . . . . . . 88

3.7 Rolling Calibration . . . . . . . . . . . . . . . . . . 89

3.7.1 Overview of Rolling Calibration . . . . . . . . . . 89 
3.7 .2 Pass 1 Calibration . . . . . . . . . . . . . 91

3.7 .3 Stability Issues $\ldots \ldots \ldots$. . . . . . . . . . . . 9 . 93

3.8 Summary . . . . . . . . . . . . . . . . . . . . . 94

4 Selection of $B \rightarrow \eta_{c} K$ Candidates $\quad 96$

4.1 Introduction . . . . . . . . . . . . . . . . 96

4.2 Data Sample. . . . . . . . . . . . . . . . . . . . . . 98

4.2 .1 Real Event Data . . . . . . . . . . . . . . 9 98

$4.2 .2 \quad$ Monte Carlo . . . . . . . . . . . . . . . . . 98

4.3 Preselection . . . . . . . . . . . . . . . . . . . . . 99

4.4 Reconstruction . . . . . . . . . . . . . . . . . 101

4.5 Candidate Selection and Optimisation _. . . . . . . . . 103

4.5.1 Overview of $B \rightarrow \eta_{c} K$ Selection $\ldots \ldots \ldots \ldots$

4.5.2 Selection Optimisation . . . . . . . . . . . . 105

4.5.3 Two-Body Decay Kinematics Selection . . . . . . . . . . 109

4.5 .4 Fisher Discriminant . . . . . . . . . . . . . . . 111

4.5.5 Charged Kaon Identification . . . . . . . . . . . . . 115

$4.5 .6 \quad K_{S}^{0}$ Selection . . . . . . . . . . . . . 117

$4.5 .7 \pi^{0}$ Selection . . . . . . . . . . . . . . 120

$4.5 .8 \eta_{c}$ Selection . . . . . . . . . . . . . . . 123

$4.5 .9 \quad$ B Selection . . . . . . . . . . . . . . . . 125

4.5.10 Resolving Multiple Candidates . . . . . . . . . . . . . 126 
4.5 .11 Fiducial Selections _. . . . . . . . . . . . . . 127

4.5.12 Summary of Optimisation . . . . . . . . . . . 127

4.6 Summary of Selections . . . . . . . . . . . . . . . . 128

5 Measurement of Branching Fraction for $B \rightarrow \eta_{c} K \quad 130$

5.1 Introduction . . . . . . . . . . . . . . . . 130

5.2 Monte Carlo Efficiency Measurement . . . . . . . . . . . . . . 132

5.2.1 Efficiency in the $\eta_{c} \rightarrow K \bar{K} \pi$ Dalitz Plot $\ldots \ldots \ldots 133$

5.2.2 An Alternative Representation of the Dalitz Plot . . . . . 137

5.2 .3 Efficiency Parametrization . . . . . . . . . . . . . . 139

5.2.4 Efficiency Parametrization Validation . . . . . . . . . . . . 142

5.2.5 Sensitivity of the Efficiency Parametrization . . . . . . . 145

5.3 Monte Carlo Efficiency Corrections . . . . . . . . . . . . . 147

5.3.1 Tracking Efficiency Corrections . . . . . . . . . . 148

$5.3 .2 \quad K_{s}^{0}$ Efficiency Corrections $\ldots \ldots \ldots \ldots . \ldots \ldots$

$5.3 .3 \quad \pi^{0}$ Efficiency Corrections . . . . . . . . . . . . . 151

5.3.4 Particle Identification Efficiency Corrections . . . . . . . . 152

$5.3 .5 \eta_{c}$ Width Uncertainty . . . . . . . . . . . . . . . . 154

5.3.6 Selection Variable Distributions . . . . . . . . . . 155

5.3.7 Self-Combinatorial Background . . . . . . . . . 159

$5.3 .8 \quad$ Summary . . . . . . . . . . . . . . . . . . . . 159

5.4 Background Estimation . . . . . . . . . . . . . . 160 
5.4 .1 Combinatorial Background . . . . . . . . . . 161

5.4 .2 Peaking Background . . . . . . . . . . . . 162

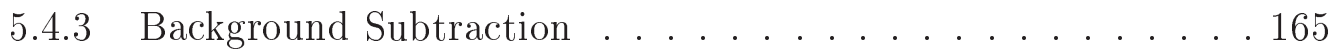

5.4.4 Validation of Background Subtraction . . . . . . . . 165

$5.5 \quad$ B Meson Counting ........................... 169

5.6 Branching Fractions . . . . . . . . . . . . 170

5.6.1 Signal Observations and Preliminary Yields . . . . . . 170

5.6.2 Branching Fraction Measurement Method . . . . . . . . . 176

5.6 .3 Systematic Errors . . . . . . . . . . . . . 179

5.6.4 Branching Fraction Results ............. 185

5.7 Conclusion . . . . . . . . . . . . . . . . 186

A Isospin Decomposition of $\eta_{c} \rightarrow K \bar{K} \pi$ Final States 191

B Curve Fitting Using Orthogonal Polynomials 194

C Alternative Representation of Phase Space in $\eta_{c} \rightarrow K \bar{K} \pi$ Decay 197

$\begin{array}{ll}\text { D Event-Shape Variables } & 201\end{array}$

E Fast Monte Carlo (PravdaMC) 205 


\section{Abstract}

The branching fraction is measured for the decay channels $B^{0} \rightarrow \eta_{c} K_{S}^{0}$ and $B^{+} \rightarrow$ $\eta_{c} K^{+}$where $\eta_{c} \rightarrow K \bar{K} \pi$, using the BABAR detector. The $\eta_{c} \rightarrow K_{S}^{0} K^{+} \pi^{-}$and $\eta_{c} \rightarrow K^{+} K^{-} \pi^{0}$ decay channels are used, including non-resonant decays and possibly those through intermediate resonances. The individual branching fractions are found to be

$$
\begin{aligned}
\mathcal{B}\left(B^{0} \rightarrow \eta_{c} K^{0}\right) \times \mathcal{B}\left(\eta_{c} \rightarrow K^{0} K^{+} \pi^{-}\right) & =(4.36 \pm 0.70 \pm 0.66) \times 10^{-5} \\
\mathcal{B}\left(B^{+} \rightarrow \eta_{c} K^{+}\right) \times \mathcal{B}\left(\eta_{c} \rightarrow K^{0} K^{+} \pi^{-}\right) & =(4.45 \pm 0.46 \pm 0.42) \times 10^{-5} \\
\mathcal{B}\left(B^{0} \rightarrow \eta_{c} K^{0}\right) \times \mathcal{B}\left(\eta_{c} \rightarrow K^{+} K^{-} \pi^{0}\right) & =(1.24 \pm 0.34 \pm 0.19) \times 10^{-5} \\
\mathcal{B}\left(B^{+} \rightarrow \eta_{c} K^{+}\right) & \times \mathcal{B}\left(\eta_{c} \rightarrow K^{+} K^{-} \pi^{0}\right)=(1.17 \pm 0.22 \pm 0.12) \times 10^{-5},
\end{aligned}
$$

where the first error is statistical and the second is systematic. The individual branching fractions are combined to obtain

$$
\begin{aligned}
\mathcal{B}\left(B^{0} \rightarrow \eta_{c} K^{0}\right) & =(1.24 \pm 0.17 \pm 0.19 \pm 0.38) \times 10^{-3} \\
\mathcal{B}\left(B^{+} \rightarrow \eta_{c} K^{+}\right) & =(1.23 \pm 0.11 \pm 0.12 \pm 0.38) \times 10^{-3},
\end{aligned}
$$

where the first error is statistical, the second is systematic, and the third is the error on the $\eta_{c} \rightarrow K \bar{K} \pi$ branching fraction. A method of calibration for the BABAR electromagnetic calorimeter using $\pi^{0} \rightarrow \gamma \gamma$ events was developed and its preliminary performance is studied. 


\section{Declaration}

No portion of the work referred to in this thesis has been submitted in support of an application for another degree or qualification of this or any other university or other institute of learning.

Copyright in text of this thesis rests with the Author. Copies (by any process) either in full, or of extracts, may be made only in accordance with instructions given by the Author and lodged in the John Rylands University Library of Manchester. Details may be obtained from the Librarian. This page must form part of any such copies made. Further copies (by any process) of copies made in accordance with such instructions may not be made without the permission (in writing) of the Author.

The ownership of any intellectual property rights which may be described in this

thesis is vested in the University of Manchester, subject to any prior agreement to the contrary, and may not be made available for use by third parties without the written permission of the University, which will prescribe the terms and conditions of any such agreement.

Further information on the conditions under which disclosures and exploitation may take place is available from the Head of the Department of Physics and Astronomy. 


\section{The Author}

Frank Jackson was educated at Ehenside and Whitehaven Schools, Cumbria, between 1988 and 1995, before obtaining a M.Sci (Hons) degree in Physics at the Imperial College of Science, Technology and Medicine in 1999. The work presented in this thesis was conducted at the University of Manchester and the Stanford Linear Accelerator Centre, California. 


\section{Author's Contribution}

The measurement of the branching fraction for the decay $B \rightarrow \eta_{c} K$ where $\eta_{c} \rightarrow$ $K_{S}^{0} K^{+} \pi^{-}$or $\eta_{c} \rightarrow K^{+} K^{-} \pi^{0}$ is described in Chapters 4 and 5. Chapter 4 describes the development and optimisation, by the author, of the selection of $B \rightarrow \eta_{c} K, \eta_{c} \rightarrow$ $K^{+} K^{-} \pi^{0}$ channels in the BABAR data sample. Some of the selection strategy was proposed and developed by others in the Charmonium Working Group at BABAR, whose contributions are acknowledged in the text of the thesis. The set of selections developed for the $B \rightarrow \eta_{c} K, \eta_{c} \rightarrow K_{S}^{0} K^{+} \pi^{-}$channel were not optimised by the author, but in a parallel analysis in the Charmonium Working Group at BABAR.

The branching fraction measurements in the decay channels $B \rightarrow \eta_{c} K, \eta_{c} \rightarrow$ $K_{S}^{0} K^{+} \pi^{-}$and $B \rightarrow \eta_{c} K, \eta_{c} \rightarrow K^{+} K^{-} \pi^{0}$ described in Chapter 5 were carried out solely by the author. The methods used to account for the impact of possible $\eta_{c} \rightarrow K^{*} \bar{K} \rightarrow K \bar{K} \pi$ decays on the measured branching fractions were proposed within the Charmonium Working Group, and are acknowledged in the text of the thesis. The evaluation of several systematic errors in the branching fraction uses several standard recipes developed by the Charmonium Working Group. Some of the recipes were implemented by others within the Charmonium Working group and these contributions are acknowledged in the text of the thesis.

The method of calibration using $\pi^{0} \rightarrow \gamma \gamma$ events was developed by R. J. Barlow.

Chapter 3 describes a method of background subtraction in the $\pi^{0} \rightarrow \gamma \gamma$ mass distribution, developed by the author, central to the calibration method. Studies of the performance of the calibration, and its possible inclusion in the automatic event reconstruction at $B A B A R$ were performed by the author and are presented here. 


\section{Acknowledgements}

I would like to thank Roger Barlow for his supervision and guidance during the last few years, for putting me on the right tracks and seeing I didn't go off them.

Two people to whom I owe great debts of gratitude are James Weatherall and Jamie Boyd who looked after me for a large part of my time at SLAC.

I certainly would have got nowhere with my studies had it not been for the help of my analysis group at SLAC. So a big thank you to Nick Barlow, Phil Clark, Gautier Hamel de Monchenault, Witold Kozanecki, Melanie Langer, Georges London, Chris Marker, Stefania Ricciardi, and Gabriella Sciolla. I am particularly grateful to Bill Dunwoodie for his input towards the end of my thesis studies. I greatly enjoyed being part of the analysis team, and the constant support I received from them gave me confidence and pride in my work.

Others who have helped in more ways than one at Manchester and SLAC include John Back, Wahid Bhimji, Steve Dallison, Alessandra Forti, Gavin Hesketh, Dave Mercer, George Lafferty, Fred Loebinger, Sabah Salih and Jenny Williams. Other friends and acquaintances - too many to name - I made in the last few years have been equally important.

The last thank you should go to my family whose continued nurturing has lead to all my achievements. 
To my parents 


\section{1 \\ Theory}

\section{$1.1 \quad$ Introduction}

In this chapter some of the theoretical aspects of $B$ meson physics are outlined. The areas discussed include those relevant to the experimental goals of the BABAR experiment and the analysis of $B \rightarrow \eta_{c} K$ branching fractions presented in Chapters 4 and 5 .

Firstly, an overview of $C P$ violation in the $B$ meson system is given. This is pertinent 
to the analysis of $B^{0} \rightarrow \eta_{c} K_{S}^{0}$ decays since this channel is expected to exhibit clean time-dependent $C P$ asymmetries. Then the methodology used to predict the branching fractions for $B \rightarrow \eta_{c} K$ decays are discussed.

\section{$1.2 \quad C P$ Violation}

The violation of charge-parity $(C P)$ symmetry is thought to be a central ingredient in the evolution of a matter dominated universe. It was initially proposed by Sakharov that in addition to baryon-number violating processes, $C P$ symmetry would also need to be violated in order for these processes to result in a universe with non-zero net baryon number [1]. Thus, observations of $C P$ violation are essential to the development and validation of a theoretical framework which incorporates baryogenesis.

The first observation of $C P$ violation was made in 1964 in the decays of neutral kaons [2]. Since then the attention has turned to heavier mesons for signs of $C P$ asymmetry. The BABAR experiment (Chapter 2) was designed to search for $C P$ violation in the $B$ meson system.

As will be seen, the Standard Model of particle physics incorporates $C P$ violation in weak interactions. Other extensive reviews of $C P$ violation in the $B$ meson system exist (see for example [3], [4]) and only an overview is given in the following sections.

\subsection{Introduction to the $B$ Meson System}

Firstly some basic properties of $B$ mesons should be described, which are crucial to the understanding of Standard Model $C P$ violation in the $B$ meson system. In 
particular, the phenomenon of meson mixing shall be seen as a mechanism by which $C P$ violation can occur.

Meson mixing is the process by which a neutral meson transforms into its antiparticle conjugate. This is possible in the neutral $B$ meson system through a second order weak transition, as shown in Figure 1.1. Neutral $B$ mesons therefore exist in a mixed

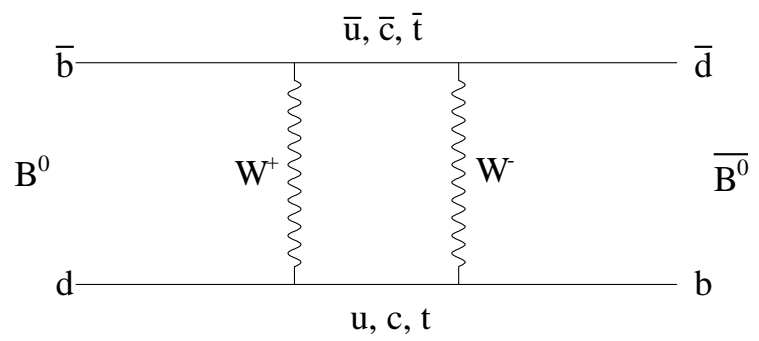

Figure 1.1: Feynman diagram for $B^{0} \rightarrow \bar{B}^{0}$ transition.

state at any given time, containing components of $B^{0}$ and $\bar{B}^{0}$ states. This mixed state can be described by

$$
\left|\phi_{B}\right\rangle=a\left|B^{0}\right\rangle+b\left|\bar{B}^{0}\right\rangle,
$$

where $a$ and $b$ carry the time dependence of the $\left|B^{0}\right\rangle$ and $\left|\bar{B}^{0}\right\rangle$ amplitudes. The Schrödinger equation for this mixed state is given by

$$
i \frac{\partial}{\partial t}\left|\phi_{B}\right\rangle=\widehat{H}\left|\phi_{B}\right\rangle=(m-i \Gamma / 2)\left|\phi_{B}\right\rangle,
$$

where $m$ and $\Gamma$ denote the mass and decay width of the decaying mixed state respectively. Applying $\left\langle B^{0}\right|$ and $\left\langle\bar{B}^{0}\right|$ to (1.2) yields two equations describing the coupled time evolution of the $B^{0}$ and $\bar{B}^{0}$ amplitudes.

$$
\begin{aligned}
i \frac{\partial}{\partial t} a & =M_{11} a+M_{12} b, \\
i \frac{\partial}{\partial t} b & =M_{21} a+M_{22} b,
\end{aligned}
$$

where $M_{i j}=m_{i j}-i \Gamma_{i j} / 2$. 
$M_{11}=\left\langle B^{0}|\widehat{H}| B^{0}\right\rangle=\left\langle\bar{B}^{0}|\widehat{H}| \bar{B}^{0}\right\rangle=M_{22}$ due to $C P T$ invariance. If $C P$ symmetry holds, $M_{12}=\left\langle\bar{B}^{0}|\widehat{H}| B^{0}\right\rangle=\left\langle B^{0}|\widehat{H}| \bar{B}^{0}\right\rangle=M_{21}$. The decoupled wavefunction superpositions satisfying

$$
\begin{aligned}
i \frac{\partial}{\partial t}\left|B_{H}\right\rangle & =\left(m_{H}-i \Gamma_{H} / 2\right)\left|B_{H}\right\rangle, \\
i \frac{\partial}{\partial t}\left|B_{L}\right\rangle & =\left(m_{L}-i \Gamma_{L} / 2\right)\left|B_{L}\right\rangle,
\end{aligned}
$$

are obtained by finding the normal modes of (1.3), which yields (not requiring $C P$ invariance)

$$
\begin{aligned}
& \left|B_{H}\right\rangle=p\left|B^{0}\right\rangle+q\left|\bar{B}^{0}\right\rangle, \\
& \left|B_{L}\right\rangle=p\left|B^{0}\right\rangle-q\left|\bar{B}^{0}\right\rangle,
\end{aligned}
$$

where $|p|^{2}+|q|^{2}=1$ and $p / q=\sqrt{\frac{\left\langle\bar{B}^{0}|\widehat{H}| B^{0}\right\rangle}{\left\langle B^{0}|\hat{H}| \bar{B}^{0}\right\rangle}} \cdot\left|B_{H}\right\rangle$ and $\left|B_{L}\right\rangle$ are the mass eigenstates of the neutral $B$ system ( $H$ denotes 'heavy' and $L$ denotes 'light'), and clearly do not have definite flavour. The mass difference between the two states $\Delta m_{B} / \Gamma_{B}=$ $m_{H}-m_{L} / \Gamma_{B}$ has been measured to be $0.755 \pm 0.015$ [5]. The width difference $\Delta \Gamma_{B}=\Gamma_{H}-\Gamma_{L}$ has not been measured, but is expected to be negligible, since the available phase space is very large in $B$ decays. It is estimated that $\Delta \Gamma_{B} / \Gamma_{B}=$ $\mathcal{O}\left(10^{-2}\right)[3]$.

As stated, $C P$ symmetry has not been assumed and thus $p \neq q$ is allowed, so the mass eigenstates $\left|B_{H}\right\rangle$ and $\left|B_{L}\right\rangle$ may not be $C P$ eigenstates. It is worth mentioning that in the neutral kaon system, the analogous pair of mass eigenstates are the $K_{S}^{0}$ and $K_{L}^{0}$ particles, which have a small mass difference but very different lifetimes. This is due to the fact that the decay channels for kaons are much more limited than for the heavier $B$ mesons, and the $C P$ allowed decays $K_{S}^{0} \rightarrow \pi \pi, K_{L}^{0} \rightarrow \pi \pi \pi$ are very differently favoured by phase space. This separation of lifetimes makes the experimental identification of the kaon mass eigenstates easier, which enabled a clear observation of $C P$ violation in the decays of neutral kaons (see Section 1.4.1).

To understand the ways in which $C P$ violation can occur, it is necessary to develop 
a model for the flavour evolution of neutral $B$ mesons, incorporating mixing and decay. A mixed, time-evolving $B$ meson state can be expressed as superposition of $\left|B_{H}\right\rangle$ and $\left|B_{L}\right\rangle$,

$$
\left|\phi_{B}(t)\right\rangle=a_{H}(t)\left|B_{H}\right\rangle+a_{L}(t)\left|B_{L}\right\rangle \text {. }
$$

Substituting expressions for $\left|B_{H}\right\rangle$ and $\left|B_{L}\right\rangle$ and applying initial conditions leads to expressions for the time dependence of states starting as pure $\left|B^{0}\right\rangle$ or $\left|\bar{B}^{0}\right\rangle$, which are

$$
\begin{aligned}
\left|B^{0}(t)\right\rangle & =f(t) \cos \left(\frac{\Delta m_{B}}{2} t\right)\left|B^{0}\right\rangle+i \frac{q}{p} f(t) \sin \left(\frac{\Delta m_{B}}{2} t\right)\left|\bar{B}^{0}\right\rangle, \\
\left|\bar{B}^{0}(t)\right\rangle & =i \frac{p}{q} f(t) \sin \left(\frac{\Delta m_{B}}{2} t\right)\left|B^{0}\right\rangle+f(t) \cos \left(\frac{\Delta m_{B}}{2} t\right)\left|\bar{B}^{0}\right\rangle,
\end{aligned}
$$

where $f(t)$ describes the decaying state and is given by

$$
f(t)=e^{-i\left(m_{H}+m_{L}\right) t / 2} e^{-\Gamma t / 2},
$$

where $\Gamma_{H}=\Gamma_{L}=\Gamma$ has been assumed. The sine and cosine factors describe the $B$ mixing. With the time evolution equations established, it is possible to study the manifestations of $C P$ violation in $B$ decays.

\section{4 $C P$ Violation in the $B$ Meson System}

\subsubsection{Comparison with the Neutral Kaon System}

The effect of $C P$ violation in $B$ decays is more subtle than in neutral kaon decays. As mentioned the two mass eigenstates $K_{S}^{0}$ and $K_{L}^{0}$ are clearly discernible by the large difference in their lifetimes; their assumed $C P$ quantum numbers were assigned by their observed decays to states of definite $C P, K_{S} \rightarrow \pi \pi(C P=1)$ and $K_{L} \rightarrow \pi \pi \pi$ $(C P=-1)$. The observation of the decay $K_{L} \rightarrow \pi \pi$ was therefore a patent violation of $C P$ symmetry . 
An analogous discovery is not possible in the $B$ meson system, where the mass eigenstates are not experimentally separable by lifetime. The effects of $C P$ violation are not expected to be recorded as the observation of a $C P$ forbidden decay. Described below are the ways in which $C P$ violation may occur and be observed.

\subsubsection{Types of $C P$ Violation in the $B$ Meson System}

\section{$C P$ Violation in Decay}

This is also called direct $C P$ violation, exhibited when the decay rate of the $B$ meson to a final state $f,\left|A_{f}\right|^{2}$, differs from the $C P$ conjugate decay $\left|\bar{A}_{\bar{f}}\right|^{2}$. The quantum mechanical amplitude $A_{f}$ is a sum of all the amplitudes that contribute to the decay, with each amplitude in the sum having its own phase. The phase can be separated into two parts; the weak phase that arises from complex weak coupling factors in the decay matrix element, and the strong phase that is due to final state interactions. The weak phases originate from the CKM matrix of the Standard Model weak interaction (see Section 1.5). These phases change sign under the action of $C P$. Final state interactions are predominantly strong and so these phases do not change sign. The phase of the total amplitude therefore is not invariant

under a $C P$ transformation, which leads to a difference in $\left|A_{f}\right|^{2}$ and $\left|\bar{A}_{\bar{f}}\right|^{2}$. At least two contributing transition amplitudes are required with different weak phases, that in addition have different strong phases.

Direct $C P$ violation has only been observed in the decays of neutral kaons to $\pi \pi$, where it is made possible through the isospin components of the $\pi \pi$ states which provide the channels by which the interference described above can decay [3]. 


\section{$C P$ Violation in Mixing}

Also called indirect $C P$ violation, this arises when the mass eigenstates are not $C P$ eigenstates, or $p \neq q$ in (1.5). The first discovery of $C P$ violation was through the decay $K_{L}^{0} \rightarrow \pi \pi$ which indicated that the mass eigenstate $K_{L}^{0}$ was not a pure $C P$ eigenstate as assumed in $C P$ symmetric $K^{0}$ mixing.

This type of $C P$ violation may be observed in semi-leptonic $B$ decays, where it would result in an asymmetry

$$
a_{s l}=\frac{\Gamma\left(\bar{B}^{0}(t) \rightarrow l^{+} \nu X\right)-\Gamma\left(B^{0}(t) \rightarrow l^{-} \nu X\right)}{\Gamma\left(\bar{B}^{0}(t) \rightarrow l^{+} \nu X\right)+\Gamma\left(B^{0}(t) \rightarrow l^{-} \nu X\right)}=\frac{1-|q / p|^{4}}{1+|q / p|^{4}}
$$

The asymmetry is expected to be small (since $|q / p| \approx 1$, see for example [3]) and is difficult to relate to fundamental Standard Model parameters.

\section{$C P$ Violation in the Interference Between Mixing and Decay}

A third type of $C P$ violation is possible which may occur even if there is no $C P$ violation in decay or mixing. It is observable only in $B$ decays to $C P$ eigenstates $\left(f_{C P}\right)$, and is measured by the difference in decay rates from time-evolving neutral $B$ states starting as pure $B^{0}$ and $\bar{B}^{0}$.

The time-dependent decay rates can be calculated following the formalism set out in (1.7), and are given by

$$
\begin{aligned}
& \Gamma\left(B^{0}(t) \rightarrow f_{C P}\right) \propto\left|\left\langle f_{C P}|\widehat{H}| B^{0}(t)\right\rangle\right|^{2}= \\
& \quad\left|A_{f_{C P}}\right|^{2} e^{-\Gamma t}\left[\frac{1+|\lambda|^{2}}{2}-\frac{1-|\lambda|^{2}}{2} \cos \left(\Delta m_{B} t\right)-\operatorname{Im}(\lambda) \sin \left(\Delta m_{B} t\right)\right],
\end{aligned}
$$

and

$$
\begin{aligned}
& \Gamma\left(\bar{B}^{0}(t) \rightarrow f_{C P}\right) \propto\left|\left\langle f_{C P}|\widehat{H}| \bar{B}^{0}(t)\right\rangle\right|^{2}= \\
& \quad\left|A_{f_{C P}}\right|^{2} e^{-\Gamma t}\left[\frac{1+|\lambda|^{2}}{2}-\frac{1-|\lambda|^{2}}{2} \cos \left(\Delta m_{B} t\right)+\operatorname{Im}(\lambda) \sin \left(\Delta m_{B} t\right)\right]
\end{aligned}
$$


where $\lambda=\eta_{f_{C P}} \frac{q}{p} \frac{\bar{A}_{f_{C P}}}{A_{f_{C P}}}, A_{f_{C P}}$ and $\bar{A}_{f_{C P}}$ are the decay amplitudes of $\left|B^{0}\right\rangle$ and $\left|\bar{B}^{0}\right\rangle$ to $\left|f_{C P}\right\rangle$, and $\eta_{f_{C P}}$ is the $C P$ quantum number of $\left|f_{C P}\right\rangle$. The assumption $|q / p|=1$ has been made.

Thus the decay rates $\Gamma\left(B^{0} / \overline{B^{0}} \rightarrow f_{C P}\right)$ differ if $\operatorname{Im}(\lambda) \neq 0$, which is satisfied if there exists a residual phase between the mixing and decay amplitudes.

The time-dependent asymmetry can thus be expressed as

$$
\begin{aligned}
a(t) & =\frac{\Gamma\left(B^{0}(t) \rightarrow f_{C P}\right)-\Gamma\left(\overline{B^{0}}(t) \rightarrow f_{C P}\right)}{\Gamma\left(B^{0}(t) \rightarrow f_{C P}\right)+\Gamma\left(\overline{B^{0}}(t) \rightarrow f_{C P}\right)} \\
& =C_{f} \cos \left(\Delta m_{B} t\right)+S_{f} \sin \left(\Delta m_{B} t\right),
\end{aligned}
$$

where

$$
C_{f}=\frac{1-|\lambda|^{2}}{1+|\lambda|^{2}} \quad S_{f}=\frac{2 \operatorname{Im} \lambda}{1+|\lambda|^{2}} .
$$

If $|\lambda|=1$ in addition to $|p / q|=1$, (no $C P$ violation in mixing or decay) the asymmetry reduces to

$$
a(t)=\operatorname{Im} \lambda \sin \left(\Delta m_{B} t\right) .
$$

As will be seen, large asymmetries are observed of the simple form (1.14) in certain $B^{0}$ decays.

\subsubsection{Measuring $C P$ Violation in $B$ Meson Decays}

To measure $C P$ violation in the interference between mixing and decay it is necessary to have a method of determining the $B$ flavour at a given point in time.

At $B A B A R$ this is achieved by coherent production of $B \bar{B}$ states in $\Upsilon(4 S)$ decay, where the flavour of the two $B$ mesons is always opposite at any point in time. Thus, if the flavour of one of $B$ mesons can be determined at the time of decay, the other $B$ meson's flavour at that time is known. Tagging decays such as $B \rightarrow D l \nu$, where the charge of the lepton indicates the flavour of the $B$, enables this determination. 
A sample of events with fully reconstructed $B$ decays to $C P$ eigenstates ( $B_{C P}$ decays) can thus be examined for, in each event, an additional tagging decay which determines the flavour of the $B_{C P}$ meson at the time of the tagging decay. The tagging decay in general is not fully reconstructed, to maximise reconstruction efficiency.

The measured $B$ decay vertices, combined with the relativistic boost of the $e^{+} e^{-} \rightarrow$ $B \bar{B}$ centre-of-mass system, yield information on the decay times. Only the difference between the $B_{C P}$ and tagging decay times, $\Delta t$, is required, which may take positive or negative values. Thus the decay time distributions (1.10), (1.11) and the timedependent asymmetry (1.12) are centred about $\Delta t=0$, under the substitution $t \rightarrow \Delta t$.

$C P$ violating parameters are then extracted from fits of the predicted decay rates (1.10), (1.11) modified to include the effects of time measurement resolution, to the experimental data. Specific examples will be given in Section 1.5.2.

\subsection{The CKM Matrix and the Unitarity Triangle}

\subsubsection{Theoretical Background}

$C P$ violation in $B$ decays requires weak phases to exist in the mixing and decay amplitudes. As mentioned, the weak quark-quark interaction of Standard Model provides the complex couplings by which these phases can arise, through the CKM matrix describing quark flavour mixing.

The full Lagrangian formalism of the weak interaction will not be discussed here, for details see for example [3]. The amplitudes of weak interactions between quarks can be expressed in terms of the 4-component weak currents $J^{\mu}$. For example the 
amplitude of weak scattering of quarks $A$ and $B$ to $C$ and $D$ can represented in current-current form

$$
\mathcal{M} \propto J_{A C}^{\mu} J_{\mu}^{B D \dagger} .
$$

Here the W-boson propagator has been omitted, which is a valid approximation in the cases of low momentum transfer. The weak currents are given by

$$
J^{\mu}=(\bar{u} \bar{c} \bar{t}) \gamma^{\mu} \frac{\left(1-\gamma^{5}\right)}{2} V\left(\begin{array}{l}
d \\
s \\
b
\end{array}\right),
$$

where $(u c t),(d s b)$ are the quark wavefunctions; $\gamma^{\mu}$ are the usual Dirac 4-matrices and $\gamma^{5}=i \gamma^{0} \gamma^{1} \gamma^{2} \gamma^{3}$. $V$ is the Cabbibo-Kobayashi-Maskawa (CKM) matrix, a unitary matrix describing the mixing of the quark fields. The presence of the CKM matrix effectively means that the quark weak eigenstates are not the mass eigenstates. This scenario was incorporated into the Standard Model after the experimental observations of the rates of weak decays which violated or conserved strangeness, and comparisons of leptonic weak decays with those involving quarks (see, for example [6]). The CKM matrix may be represented

$$
V=\left(\begin{array}{ccc}
V_{u d} & V_{u s} & V_{u b} \\
V_{c d} & V_{c s} & V_{c b} \\
V_{t d} & V_{t s} & V_{t b}
\end{array}\right) .
$$

The elements of the CKM matrix are complex, and thus comprise 18 parameters in total. However the unitarity constraint $V V^{\dagger}=\mathbf{1}$ reduces the number of free parameters to 4 . These are 3 Euler angles and, crucially, one phase which cannot be removed by arbitrary redefinition of the quark field phases. It is this phase that arises in the matrix elements for weak hadron decays and allows $C P$ violation to occur.

The unitarity relation $V V^{\dagger}=\mathbf{1}$ leads to 9 equations involving the CKM matrix elements. 6 of these equations can be represented by triangles in the complex plane, and from experimental measurements of the CKM elements, the most open triangle is expected to be that corresponding to the equation

$$
V_{u d} V_{u b}^{*}+V_{c d} V_{c b}^{*}+V_{t d} V_{t b}^{*}=0 .
$$


This triangle is known as the Unitarity Triangle and is shown in Figure 1.2. The

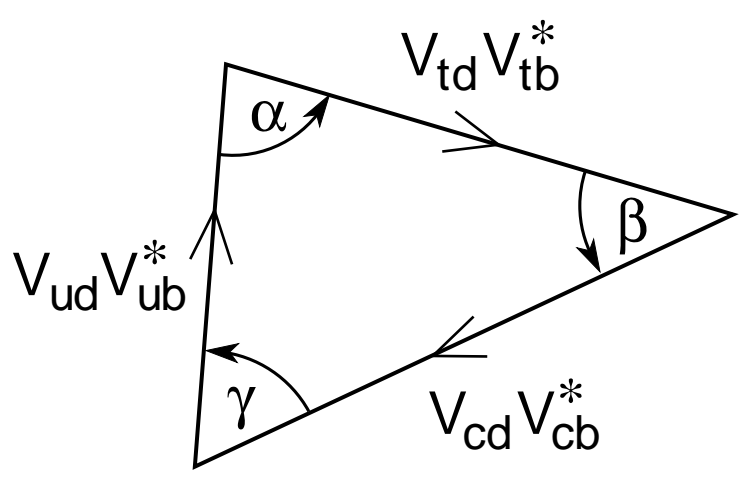

Figure 1.2: The Unitarity Triangle.

angles of the Unitarity Triangle are given by

$$
\alpha \equiv \arg \left(-\frac{V_{t d} V_{t b}^{*}}{V_{u d} V_{u b}^{*}}\right), \quad \beta \equiv \arg \left(-\frac{V_{c d} V_{c b}^{*}}{V_{t d} V_{t b}^{*}}\right), \quad \gamma \equiv \arg \left(-\frac{V_{u d} V_{u b}^{*}}{V_{c d} V_{c b}^{*}}\right) .
$$

The angles can be related to the $C P$ violating effects from the interference between mixing and decay in the $B$ system and can be measured at $B A B A R$, as is discussed in the next section.

\subsubsection{Experimental Measurements}

Measurement of each of the Unitarity Triangle angles may be achieved by measuring the decay rates of $B$ mesons to $C P$ eigenstates. The angle $\beta$ is most easily extracted from decays involving a $b \rightarrow c \bar{c} s$ transition. These are decays of the type $B^{0} \rightarrow$ $(c \bar{c}) K^{0}$ which include

$$
B^{0} \rightarrow \eta_{c} K_{S}^{0}, B^{0} \rightarrow J / \Psi K_{S}^{0}, B^{0} \rightarrow \chi_{c} K_{S}^{0}, B^{0} \rightarrow J / \Psi K_{L}^{0}, B^{0} \rightarrow J / \Psi K^{* 0} .
$$

These decays, shown in Figure 1.3 are dominated by a single tree-level diagram. Since they involve only one weak phase, the measured asymmetry reduces to that 
due only to the interference between mixing and decay (1.14).

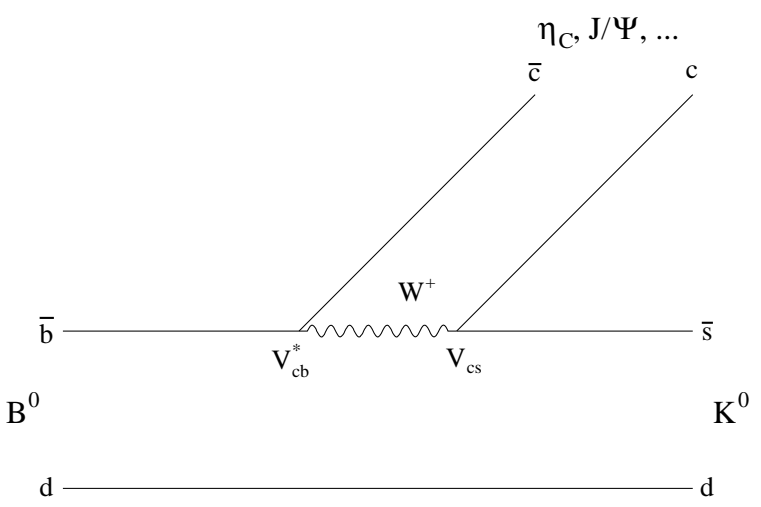

Figure 1.3: Feynman diagram for $B^{0} \rightarrow(c \bar{c}) K^{0}$ transition.

For the decays described by Figure 1.3, the $\lambda$ parameter from the time-dependent asymmetry relation (1.14) is given by

$$
\lambda\left(B^{0} \rightarrow c \bar{c} K_{S}^{0}\right)=\eta_{f_{C P}}\left(\frac{q}{p}\right)_{B} \frac{\bar{A}_{f_{C P}}}{A_{f_{C P}}}\left(\frac{q}{p}\right)_{K} .
$$

There are three ratios of amplitudes due to $B$ meson mixing, $B$ decay, and kaon mixing. These reduce to ratios of CKM matrix elements which can be read from the relevant Feynman diagrams, to give

$$
\lambda\left(B^{0} \rightarrow c \bar{c} K_{S}^{0}\right)=\eta_{f_{C P}}\left(\frac{V_{t b}^{*} V_{t d}}{V_{t b} V_{t d}^{*}}\right)\left(\frac{V_{c s}^{*} V_{c b}}{V_{c s} V_{c b}^{*}}\right)\left(\frac{V_{c d}^{*} V_{c s}}{V_{c d} V_{c s}^{*}}\right) .
$$

It follows that

$$
\operatorname{Im} \lambda\left(B^{0} \rightarrow c \bar{c} K_{S}^{0}\right)=\sin 2 \beta
$$

Thus the Unitarity Triangle angle $\beta$ is seen to enter into the time-dependent asymmetry $a(t)$. Since it enters the asymmetry as $\sin 2 \beta$, and only this parameter can be measured, a four-fold ambiguity is present in the inferred value of $\beta$. In practice the parameter $\sin 2 \beta$ is extracted from a fit to the decay rate distributions of $B^{0}$ and $\bar{B}^{0}$ mesons [7]. In the channel $B^{0} \rightarrow J / \Psi K^{* 0}$, the situation is more complicated since 
the $J / \psi K^{* 0}$ state has three angular momentum configurations, with each component having a different $P$ quantum number.

The other angles of the Unitarity Triangle cannot be measured so easily as $\beta$. Measurement of $\alpha$ is expected to be possible through the decays $B^{0} \rightarrow \pi \pi$, which does not proceed through a single weak phase, and the time-dependent asymmetry is of the more complicated form (1.12) . The angle $\gamma$ is yet more difficult to measure, but may potentially be extracted for non-time-dependent asymmetries in the decays $B^{+} \rightarrow D_{C P}^{0} K^{+}$, where $D_{C P}^{0}$ is as a $C P$ eigenstate. For more details on the proposed methods to measure $\alpha$ and $\gamma$, see [3].

The BABAR experiment has recently measured $\sin 2 \beta=0.741 \pm 0.067 \pm 0.034$ [8], clearly establishing $C P$ violation in the $B$ meson system. The measured value of $\sin 2 \beta$ was in the range of the expectations based on theory and previous experimental measurements which constrain the lengths and angles of the Unitarity Triangle [5].

\subsection{The Decays $B \rightarrow \eta_{c} K$}

As mentioned above, the time-dependent asymmetry in the decay $B^{0} \rightarrow \eta_{c} K_{S}^{0}$ can be used to measure $\sin 2 \beta$. Both the $\eta_{c}$ and $K_{S}^{0}$ are scalar particles which means the $\eta_{c} K_{S}^{0}$ state has zero angular momentum and thus is a pure $C P=-1$ eigenstate. An analysis carried out at $B A B A R$ using approximately 88 million $B \bar{B}$ decays measured $\sin 2 \beta=0.59 \pm 0.32$, using the $B^{0} \rightarrow \eta_{c} K_{S}^{0}$ channel [8].

The decays of $B \rightarrow \eta_{c} K$, like other $B$ decays to exclusive charmonium final states are colour suppressed due to their topology. Their branching fractions are small $(\mathcal{B}(B \rightarrow$ $\left.\left.\eta_{c} K\right) \sim \mathcal{O}\left(10^{-3}\right)\right)$. Predictions of the branching fractions have been made relative 
to those for $B \rightarrow J / \Psi K$, using effective theories based on the operator product expansion, utilising factorization and heavy quark symmetry. For an introduction to these methods see for example [9]. Experimental measurements of the branching fractions are therefore useful in determining the validity of these approaches.

\subsubsection{Predictions of $B \rightarrow \eta_{c} K$ Branching Fractions}

Various predictions of $B \rightarrow \eta_{c} K$ branching fractions have been made. This section attempts to summarise the common methodology behind the predictions. For a more detailed review of the theoretical background, see [3], [9]. References for specific predictions are given below.

The starting point in predicting the decay rates for $B \rightarrow \eta_{c} K$ is the formulation of an effective Hamiltonian, using the operator product expansion (OPE) for the weak $\Delta B=1$ transition. This recasts the matrix element for the $b \rightarrow c \bar{c} s$ decay, which involves a W-boson propagator and a non-local product of currents, in terms of a sum of local operators. The first term, describing four-quark interaction at a point, is given by

$$
\frac{G_{F}}{\sqrt{2}} V_{c s} V_{c b}^{*} O_{1}=\frac{G_{F}}{\sqrt{2}} V_{c s} V_{c b}^{*} \bar{s}_{i} \gamma^{\mu}\left(1-\gamma^{5}\right) c^{i} \bar{c}_{j} \gamma^{\mu}\left(1-\gamma^{5}\right) b^{j},
$$

where $G_{F}$ is the Fermi constant, and $i, j$ are the colour indicies of the quark wavefunctions. Higher corrections in the expansion are suppressed by factors of the order $m_{b}^{2} / m_{W}^{2} \sim \mathcal{O}\left(10^{-3}\right)$.

At this stage only the weak transition in Figure 1.3 has been described. A more realistic picture of the $B \rightarrow \eta_{c} K$ decay involves strong interactions between the initial and final state quarks, including many complicated exchanges of gluons. The simplest of these interactions has the effect of changing the color structure of the 
matrix element, yielding an effective Hamiltonian

$$
H_{e f f}=\frac{G_{F}}{\sqrt{2}} V_{c s} V_{c b}^{*}\left(C_{1} O_{1}+C_{2} O_{2}\right)
$$

where

$$
O_{2}=\bar{s}_{i} \gamma^{\mu}\left(1-\gamma^{5}\right) c^{j} \bar{c}_{j} \gamma^{\mu}\left(1-\gamma^{5}\right) b^{i}
$$

and $C_{1}, C_{2}$ are the Wilson coefficients. The essential feature of the OPE is that it separates the long range interactions (described by the operators $O_{1}, O_{2}$ ) from the short range effects, which are contained in the coefficients $C_{1}, C_{2}$ and calculated perturbatively (their values are independent of the external hadronic states involved in the interaction). This separation of long and short distance effects into factors that can be calculated separately is known as one kind of factorization. Without perturbative QCD corrections, $C_{1}=1, C_{2}=0$.

The matrix element for $B \rightarrow \eta_{c} K$ is then obtained from the effective Hamiltonian, and is given by

$$
\mathcal{M}=\left\langle\eta_{c} K\left|H_{e f f}\right| B\right\rangle
$$

A method of reorganising the matrix element is possible by Fierz reordering. Only the result of this technique will be given here (for details see [3]), which is

$$
\mathcal{M}=\frac{G_{F}}{\sqrt{2}} V_{c s} V_{c b}^{*}\left(C_{2}+\frac{1}{3} C_{1}\right)\left\langle\eta_{c} K\left|\bar{c} \gamma^{\mu}\left(1-\gamma^{5}\right) c \bar{s} \gamma^{\mu}\left(1-\gamma^{5}\right) b\right| B\right\rangle+\text { term }
$$

The form of this matrix element can be understood loosely from the color structure of the operators $O_{1}, O_{2}$ applied to the $B \rightarrow \eta_{c} K$ diagram. $O_{1}$ will enter the diagram suppressed by a factor of $1 / 3$, while $O_{2}$ enters unsuppressed. The term omitted is one that cannot be simplified by naive factorization.

The naive factorization ansatz allows the simplification of matrix elements like (1.27), which are re-expressed (ignoring the non-factorizable term) as

$$
\mathcal{M}=\frac{G_{F}}{\sqrt{2}} V_{c s} V_{c b}^{*} a_{2}\left\langle\eta_{c}\left|\bar{c} \gamma^{\mu}\left(1-\gamma^{5}\right) c\right| 0\right\rangle\left\langle K\left|\bar{s} \gamma^{\mu}\left(1-\gamma^{5}\right) b\right| B\right\rangle
$$


Here the convention $a_{2}=C_{2}+C_{1} / 3$ is used as in the literature. The qualitative justification of naive factorization is based on color transparency, which assumes that the $c \bar{c}$ state (the $\eta_{c}$ ) produced in the $B$ decay only interacts with the surrounding medium or quarks and gluons as a colour dipole (not as individual $c$ and $\bar{c}$ quarks), which remains small on the QCD scale until it is far from the quarks which combine to form the kaon.

The matrix element factors in (1.28) are typically re-expressed in terms of decay constants and hadronic form factors to give

$$
\mathcal{M} \propto i \mathcal{M}_{0} f_{\eta_{c}} F^{B \rightarrow K}\left(q^{2}\right),
$$

where $\mathcal{M}_{0}$ contains the Fermi constant, the CKM elements and the Wilson coefficients. $q$ is the momentum transfer, $f_{\eta_{c}}$ is the $\eta_{c}$ decay constant, and $F^{B \rightarrow K}\left(q^{2}\right)$ is the hadronic form factor for the $B \rightarrow K$ transition. The methods of determin$\operatorname{ing} f_{\eta_{c}}, F^{B \rightarrow K}\left(q^{2}\right)$ will not be described here; they include QCD sum rules and (for $\left.f_{\eta_{c}}\right)$ inter-quark potential models. In calculating the $B \rightarrow \eta_{c} K$ branching fractions, phase space factors are folded with the matrix element $\mathcal{M}$.

In the predicted ratio of branching fractions $R_{K}=\mathcal{B}\left(B \rightarrow \eta_{c} K\right) / \mathcal{B}(B \rightarrow J / \Psi K)$, the constants contained in $\mathcal{M}_{0}$ cancel. The ratio is then given by

$$
R_{K}=T \frac{f_{\eta_{c}}^{2}}{f_{J / \psi}^{2}},
$$

where $T$ contains the phase space and hadronic form factor ratios. The value of $f_{J / \psi}$ obtained from experiment is sometimes used.

Several predictions of $R_{K}$ which use the methods set out above are given in Table 1.1. The variation in the estimations arise from different techniques of calculating $T$ and $f_{\eta_{c}}^{2} / f_{J / \psi}^{2}$, although there is some interdependence of the results. While the predictions of $R_{K}$ have quite large errors, they all estimate the $B \rightarrow \eta_{c} K$ branching fraction to be of the same order of that for $B \rightarrow J / \Psi K$. 
Table 1.1: Theoretical predictions of $R_{K}$, the ratio of branching fractions $\mathcal{B}\left(B \rightarrow \eta_{c} K\right), \mathcal{B}(B \rightarrow J / \Psi K)$.

\begin{tabular}{c|c}
\hline$R_{K}$ & Ref. \\
\hline \hline $1.64 \pm 0.27$ & {$[10]$} \\
$1.6 \pm 0.2$ & {$[11]$} \\
{$[0.94,1.24]$} & {$[12]$} \\
$0.94 \pm 0.25$ & {$[13]$} \\
{$[0.97,1.28]$} & {$[14]$} \\
\hline
\end{tabular}

The theoretical predictions of $R_{K}$, along with the experimentally measured values of $\mathcal{B}\left(B \rightarrow \eta_{c} K\right), \mathcal{B}(B \rightarrow J / \Psi K)$, and $f_{J / \psi}$ could yield an estimate of the $\eta_{c}$ decay constant.

\subsubsection{The $\eta_{c}$ Meson}

The $\eta_{c}$ is the ground state $\left({ }^{2 S+1} L_{J}={ }^{1} S_{0}\right)$ of the charmonium system with quantum numbers $I^{G}\left(J^{P C}\right)=0^{+} 0^{-+}$. Its nominal mass and width are $2979.7 \pm 1.5 \mathrm{MeV} / c^{2}$ and $16_{-3.2}^{+3.6} \mathrm{MeV} / c^{2}$ respectively [5].

Unlike the vector meson $J / \psi$, the $\eta_{c}$ cannot be produced in $e^{+} e^{-}$collisions and cannot decay to lepton-antilepton pairs, due to angular momentum and $C$ conservation rules. The $\eta_{c}$ can be produced by radiative $J / \psi$ transitions, and decays hadronically via diagrams such as the one shown in Figure 1.4. Experimentally this makes the $\eta_{c}$ more difficult to observe than the $J / \psi$, and indeed was not discovered until several years after the $J / \psi[15],[16]$.

The $\eta_{c}$, like the $J / \psi$, lies below the threshold for charmed meson pair production; its 


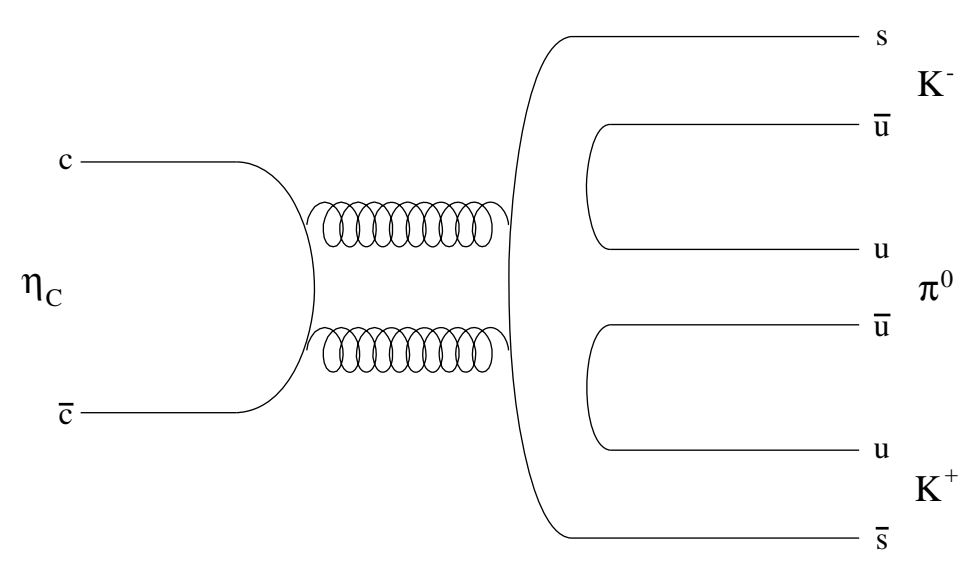

Figure 1.4: Feynman diagram for $\eta_{c} \rightarrow K^{+} K^{-} \pi^{0}$ transition.

allowed hadronic decays are of the type suppressed by the Zweig rule. Thus the $\eta_{c}$ hadronic width would be expected to be smaller than in heavy charmonium decays such as $\psi(4040) \rightarrow D \bar{D}$, but larger than in $J / \psi$ hadronic decays since the $\eta_{c}$ and $J / \psi$ decays are mediated by a minimum of 2 and 3 gluons respectively [17].

An simple estimate of the ratio of the $J / \psi$ and $\eta_{c}$ full widths (dominated by their hadronic decays) can be made based on an analogy to positronium decays to 3 or 2 photons. This gives [18]

$$
\frac{\Gamma\left(\eta_{c} \rightarrow \text { hadrons }\right)}{\Gamma(J / \psi \rightarrow \text { hadrons })}=\frac{27 \pi}{5\left(\pi^{2}-9\right)} \frac{\alpha_{s}^{2}\left(\eta_{c}\right)}{\alpha_{s}^{3}(J / \psi)} \gtrsim 80
$$

where the strong coupling constant $\alpha_{s}$ is taken to be approximately 0.2 at the charmonium mass scale $\left(\sim 3 \mathrm{GeV} / c^{2}\right)$. This gives the $\eta_{c}$ full width as $\Gamma\left(\eta_{c}\right) \gtrsim 7 \mathrm{MeV} / c^{2}$, using $\Gamma(J / \psi)=87 \mathrm{keV} / c^{2}$. More sophisticated calculations [19] predict $\Gamma\left(\eta_{c} \rightarrow\right.$ hadrons $)$ relative to $\Gamma\left(\eta_{c} \rightarrow \gamma \gamma\right)$ using first order perturbative QCD corrections to the simple rates derived in [18]. These calculations have been interpreted to estimate $\Gamma\left(\eta_{c}\right) \gtrsim 25 \mathrm{MeV} / c^{2}$ [20], [21]. A more recent estimation using fully relativistic matrix elements to describe the $c \bar{c}$ annihilation gives $\Gamma\left(\eta_{c}\right) \approx 23 \mathrm{MeV} / c^{2}[22]$. 
The world-average $\eta_{c}$ full width quoted above is the average of several widely different measurements, ranging from $7.0_{-7.0}^{+7.5} \mathrm{MeV} / c^{2}$ to $27.0 \pm 5.8 \pm 1.4 \mathrm{MeV} / c^{2}$. The most recent measurements (not included in the world-average) give $\Gamma\left(\eta_{c}\right)=29 \pm 8 \pm$ $6 \mathrm{MeV} / c^{2}[23]$ and $\Gamma\left(\eta_{c}\right)=33.3 \pm 2.5 \pm 0.8 \mathrm{MeV} / c^{2}[20]^{1}$.

Thus, the $\eta_{c}$ width is not well known, and it will be seen later that the width of the $\eta_{c}$ plays an important part in $\mathcal{B}\left(B \rightarrow \eta_{c} K\right)$ branching fraction measurements.

The $\eta_{c}$ decay modes that offer the greatest possibility of reconstruction have branching fractions at the level $\lesssim 5 \%$, such as $\eta_{c} \rightarrow K \bar{K} \pi$ and $\eta_{c} \rightarrow K^{+} K^{-} K^{+} K^{-}$. The branching fractions have been measured from $\eta_{c}$ production in radiative $J / \psi$ decays and are known only to an accuracy of about $30 \%$. This limits the accuracy at which branching fractions $\mathcal{B}\left(B \rightarrow \eta_{c} K\right)$ can be measured.

\subsection{3 $\eta_{c} \rightarrow K \bar{K} \pi$ Decays}

Branching fractions for $\eta_{c} \rightarrow K \bar{K} \pi$ have been measured [5] at

$$
\Gamma\left(\eta_{c} \rightarrow K \bar{K} \pi\right) / \Gamma_{\text {total }}=0.055 \pm 0.017 \text {. }
$$

The branching fractions of $\eta_{c}$ to specific $K \bar{K} \pi$ final states can be related by isospin symmetry, using Clebsch-Gordon coefficients (see Appendix A). This predicts (neglecting phase space factors $)^{2}$

$$
\mathcal{B}\left(\eta_{c} \rightarrow K \bar{K} \pi\right)=3 / 2 \mathcal{B}\left(\eta_{c} \rightarrow K_{S}^{0} K^{+} \pi^{-}\right)=6 \mathcal{B}\left(\eta_{c} \rightarrow K^{+} K^{-} \pi^{0}\right) .
$$

The decays $\eta_{c} \rightarrow K \bar{K} \pi$ may proceed directly, or through intermediate resonances $\eta_{c} \rightarrow K^{*} \bar{K} \rightarrow K \bar{K} \pi$. The possible resonant contributions are

$$
\eta_{c} \rightarrow K^{* 0} \bar{K}^{0} \rightarrow K^{+} \pi^{-} K_{S}^{0}
$$

\footnotetext{
${ }^{1} B A B A R$ preliminary measurement.

${ }^{2}$ Here and henceforth, charged conjugate particles are implied in the notation unless otherwise stated.
} 


$$
\begin{aligned}
& \eta_{c} \rightarrow K^{*+} K^{-} \rightarrow K_{S}^{0} \pi^{+} K^{-}, \\
& \eta_{c} \rightarrow K^{*+} K^{-} \rightarrow K^{+} \pi^{0} K^{-} .
\end{aligned}
$$

An upper limit has been placed on the $\eta_{c} \rightarrow K^{*}(890) \bar{K}$ branching fraction at $<1.28 \%$ [3] [24]. However, evidence exists for $\eta_{c} \rightarrow K^{*}(1430) \bar{K} \rightarrow K \bar{K} \pi$. In the recent $\eta_{c}$ width measurement [21] it was estimated that one third of the $\eta_{c} \rightarrow K_{S}^{0} K^{+} \pi^{-}$ candidates detected proceeded through $\eta_{c} \rightarrow K^{*}(1430) \bar{K}$.

Evidence of decays involving hadronic resonances in the three body $\eta_{c} \rightarrow K \bar{K} \pi$ decay is most clear in the Dalitz plot, which gives a diagrammatic representation of the decay rate in three-body phase space. The dynamics of three body decays dictate that the population of $\eta_{c} \rightarrow K \bar{K} \pi$ events in phase space is given by [5], [25]

$$
d N \propto|\mathcal{M}|^{2} d m_{K \pi}^{2} d m_{\bar{K} \pi}^{2},
$$

where $|\mathcal{M}|$ is the matrix element describing the decay, and $m_{K \pi}$ is the invariant mass of the $K \pi$ combination. The two-dimensional distribution of events in $m_{K \pi}^{2}, m_{\bar{K} \pi}^{2}$ is known as the Dalitz plot (it can also be represented by $m_{K \pi}^{2}$ vs. $m_{K \bar{K}}^{2}$ or $m_{\bar{K} \pi}^{2}$ vs. $m_{K \bar{K}}^{2}$ ). For non-resonant $\eta_{c} \rightarrow K \bar{K} \pi$ decays the Dalitz plot is uniformly populated, while the presence of $\eta_{c} \rightarrow K^{*} \bar{K} \rightarrow K \bar{K} \pi$ is indicated by the appearance of vertical and horizontal bands. For example in the $K_{S}^{0} K^{+} \pi^{-}$Dalitz plot $\left(m_{K_{S}^{0} \pi^{-}}^{2}\right.$ vs. $\left.m_{K^{+} \pi^{-}}^{2}\right)$, the resonant decay $\eta_{c} \rightarrow K^{*}(1430) \bar{K}$ would appear as bands at $m_{K_{S}^{0} \pi^{-}}=m_{K^{*-}(1430)}$ and $m_{K^{+} \pi^{-}}=m_{K^{* 0}(1430)}$.

The kinematic boundaries of the Dalitz plot, determined by energy-momentum conservation, are given by the maximum and minimum values of $m_{K \pi}^{2}$ for a given value of $m_{\bar{K} \pi}^{2}[5]$

$$
\begin{aligned}
\left(m_{K \pi}^{2}\right)_{\max } & =\left(E_{K}+E_{\pi}\right)^{2}-\left(p_{K}-p_{\pi}\right)^{2}, \\
\left(m_{K \pi}^{2}\right)_{\min } & =\left(E_{K}+E_{\pi}\right)^{2}-\left(p_{K}+p_{\pi}\right)^{2},
\end{aligned}
$$

where the energies $E_{K, \pi}$ and momenta $p_{K, \pi}$ are calculated in the $m_{\bar{K} \pi}$ rest frame. The limits are determined when the momenta $\mathbf{p}_{K}, \mathbf{p}_{\pi}$ are aligned or anti-aligned. The 
shape described by the boundary in the Dalitz plot is non-rectilinear, as can be seen in Figure 1.5 which shows the kinematically allowed region in the $\eta_{c} \rightarrow K_{S}^{0} K^{+} \pi^{-}$ phase space.

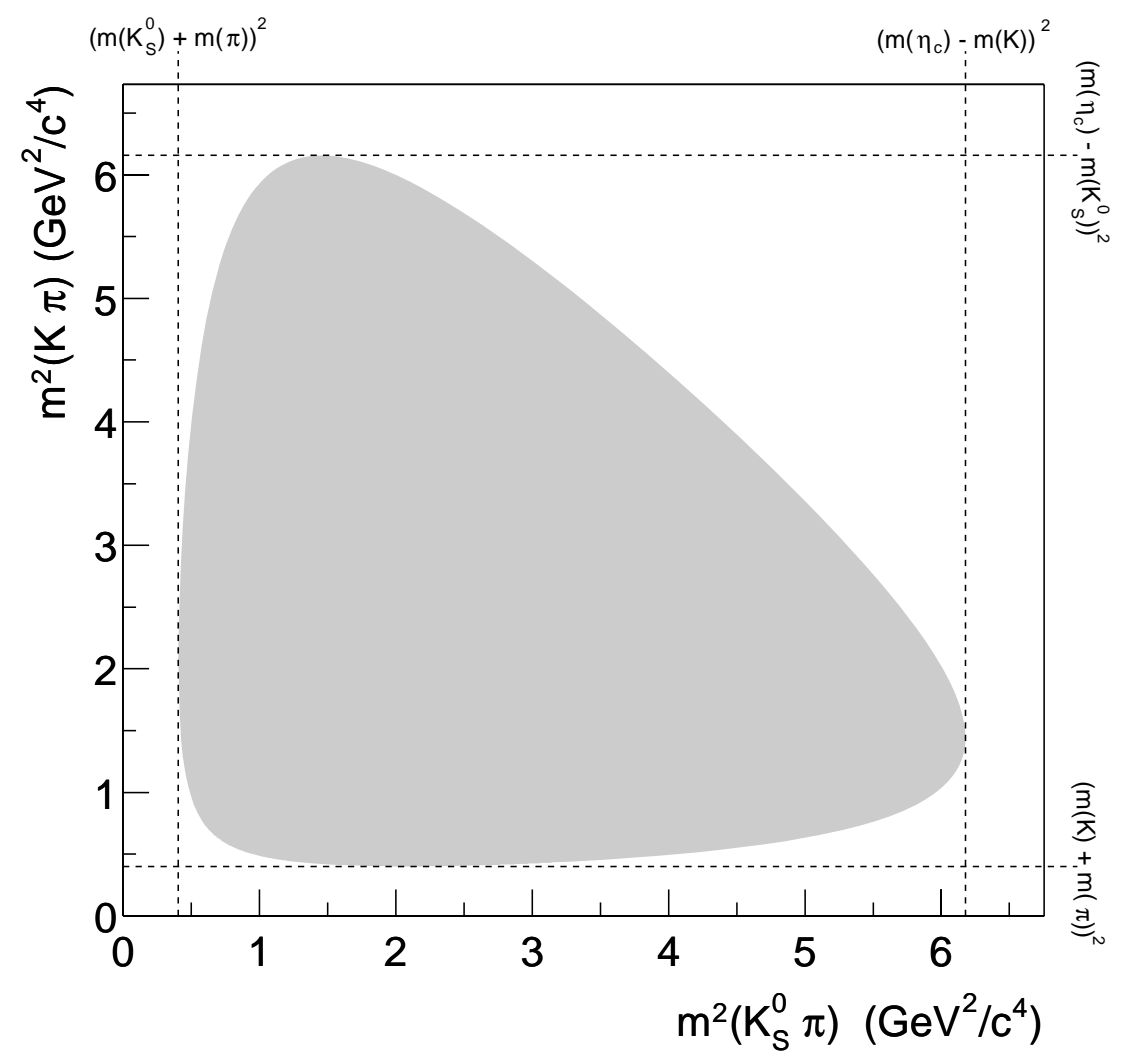

Figure 1.5: Illustration of the Dalitz Plot for the $\eta_{c} \rightarrow K_{S}^{0} K^{+} \pi^{-}$decay. The grey region represents the allowed occupancy values for $\eta_{c} \rightarrow K_{S}^{0} K^{+} \pi^{-}$events, dictated by three-body phase space.

The phase space represented by the Dalitz plot described above may be described by other kinematic variables. For instance it may be shown that the invariant mass of one two-body system is related to the angular distribution of the other two-body system (for a given value of the other two-body mass). The details of this alternative representation of the three-body phase space are given in Appendix C. While the 
conventional Dalitz plot described above offers the best illustration of masses of intermediate resonances, other phase space representations may be more useful if the experimental $\eta_{c}$ reconstruction efficiency measurement across the phase space is required. It can be shown

$$
d m_{K \pi}^{2} d m_{\bar{K} \pi}^{2} \rightarrow C d m_{K \pi} d \cos \theta,
$$

where $\theta$ is known as the helicity angle of the $(K \pi)$ system defined as the angle between the pion (or kaon) direction in the $(K \pi)$ frame and the $(K \pi)$ direction in the $\eta_{c}$ frame; $\cos \theta$ is known as the helicity cosine and $C$ is a factor describing the variable transformation. The helicity cosine is independent of $m_{K \pi}$ and thus the kinematic limits in $d m_{K \pi}, d \cos \theta$ space are orthogonal. The treatment of the $\eta_{c}$ decay phase space in the context of the $B \rightarrow \eta_{c} K$ branching fraction will be discussed in Chapter 5. 


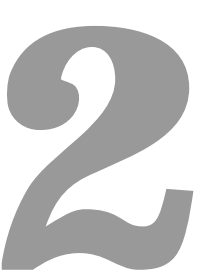

\section{The $B A B A R$ Experiment}

The main goal of the $B A B A R$ experiment is to measure $C P$ violation in the $B$ meson system, including the time-dependent asymmetries discussed in Chapter 1. Production of $B$ mesons at $B A B A R$ is achieved using an electron positron collider running at the $\Upsilon(4 S)$ resonance, in which the energies of the incident electrons and positrons are unequal to enable measurement of time-dependent asymmetries. The method of producing $B$ mesons via the $\Upsilon(4 S)$ resonance has lead to the term $B$-Factory being used to describe the experiment.

For $C P$ violation measurements a large sample of $B$ meson decays is required, since 
only a small subset will include the transitions in which $C P$ violation may be manifest; of these, yet a smaller sample will be detectable. Typically, the relevant decay branching fractions are of the order $10^{-3}$ and efficiency of decay reconstruction can vary, depending on the topology of the decay. A high collision luminosity is required, in combination with the best possible performance of tracking and calorimetric detector components, and effective techniques of particle identification.

\subsection{The PEP-II Storage Rings and Interaction Point}

The electrons and positrons which undergo collisions are provided by SLAC (Stanford Linear Accelerator Centre) linear accelerator and are stored in the PEP-II (Positron-Electron Project) storage rings at $9.0 \mathrm{GeV}$ and $3.1 \mathrm{GeV}$ respectively. This provides a collision centre-of-mass energy of $10.58 \mathrm{GeV}$, corresponding to the $\Upsilon(4 S)$ resonance. The effective cross-sections for the physics processes from $e^{+} e^{-}$interactions at the $\Upsilon(4 S)$ resonance are shown in Table 2.1. The ratio of $b$ quark production to that of all quark types is around 0.25 . Due to the asymmetric positron and electron energies the $\Upsilon(4 S)$ resonance is moving in the detector frame, such that the daughter $B$ mesons have measurable decay lengths, which would not be the case with a symmetric arrangement.

The electrons beams are stored in the high energy ring (HER) while the positrons are stored in the low energy ring (LER). The beams consist of many closely spaced bunches (typically 553-829 bunches per ring [26]) which collide head on at the interaction region shown in Figure 2.1. The typical beam currents are 0.7A for the HER and 1.3A for the LER [26]. The beams are separated in the horizontal direction by dipole magnets (B1), to prevent collisions at any position other than the interaction 
Table 2.1: Production cross-sections at the $\Upsilon(4 S)$ resonance, taken from [26].

These are effective cross-sections which take into account experimental acceptance. The production rate is calculated for a luminosity of $3 \times 10^{33} \mathrm{~cm}^{-2} \mathrm{~s}^{-1}$.

\begin{tabular}{c|c|c}
\hline$e^{+} e^{-} \rightarrow$ & Cross-section (nb) & Production Rate $(\mathrm{Hz})$ \\
\hline \hline$b \bar{b}$ & 1.1 & 3.2 \\
other $q \bar{q}$ & 3.4 & 10.2 \\
\hline$\tau^{+} \tau^{-}$ & 0.9 & 2.8 \\
$\mu^{+} \mu^{-}$ & 1.2 & 3.5 \\
$e^{+} e^{-}$ & $\sim 53$ & 159 \\
\hline
\end{tabular}

point; focusing of the beam is effected by a series of quadrupole magnets. The interaction region is enclosed by a $27.8 \mathrm{~mm}$ beryllium beam pipe, whose total thickness at the central section is equivalent to $1.06 \%$ of a radiation length, at normal incidence. The angle of the colliding beams is offset by $20 \mathrm{mrad}$ relative to the detector axis to minimise beam orbit perturbations by the $1.5 \mathrm{~T}$ solenoidal field.

There are several backgrounds originating from the beam. The dipole magnets cause intense synchrotron radiation (fan radiation) to be emitted from the beam at each side of the interaction point. However, the geometry of the collision region is designed so that most of this radiation is channelled through the detector without interacting. To attenuate fan radiation, radiation masks are used and the beam pipe is coated with gold on its inner surface and wrapped with foil on both sides of the interaction point. Background also arises from interaction between beam particles and gas in the beam pipe, including bremsstrahlung and multiple scattering; resulting degraded energy particles are swept into the detector by the dipole magnets. This type of background is minimised by maintaining a high vacuum in the beam pipe. 


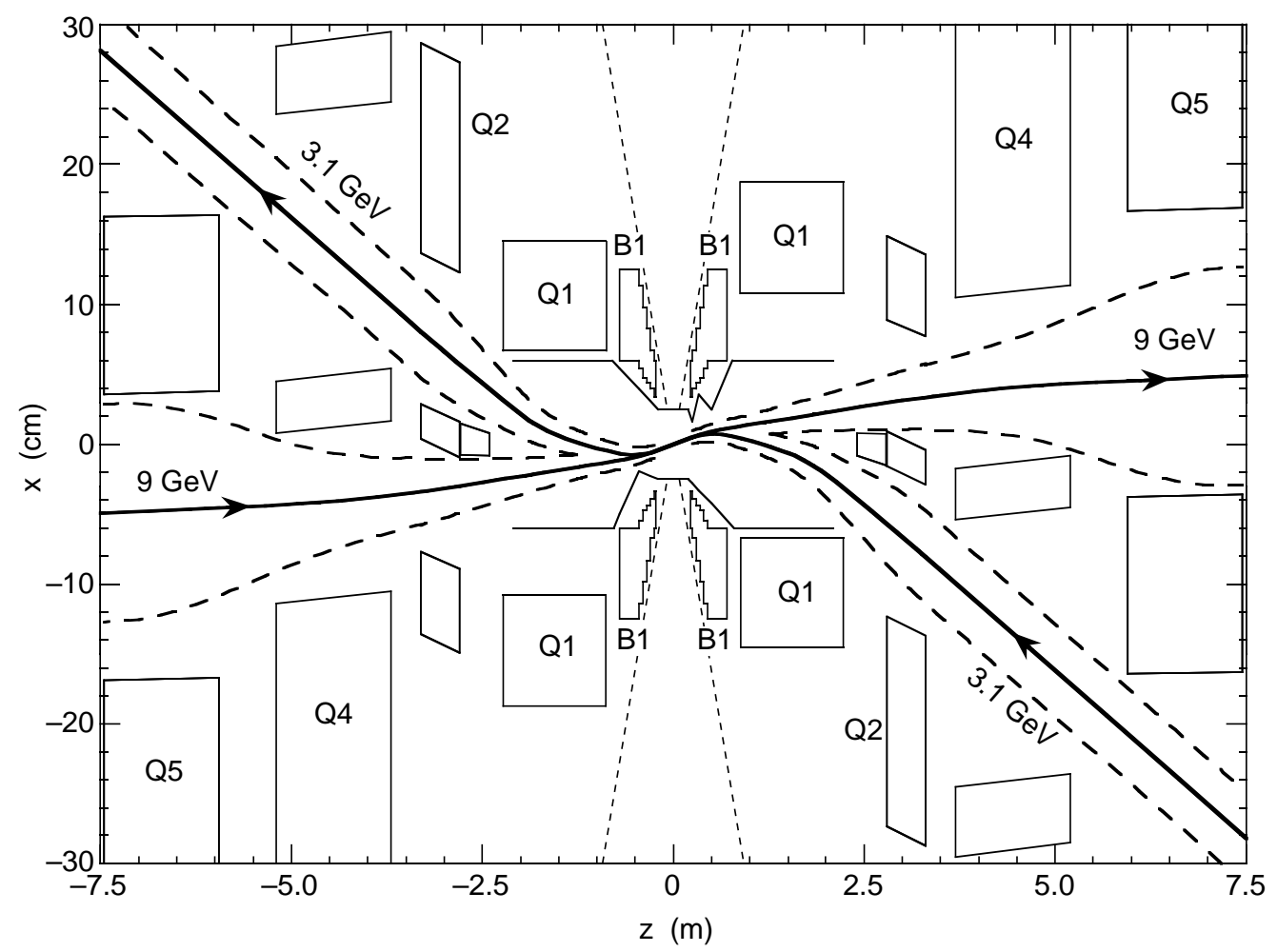

Figure 2.1: Interaction region schematic. The beams are separated by dipole magnets $B 1$ at $\pm 21 \mathrm{~cm}$ from the interaction point. Beam focusing is provided by quadrupole magnets Q1-5. The dashed lines represent the beam stay-clear regions and the 300 mrad detector acceptance. The scale on the vertical axis is highly enlarged.

The PEP-II machine has met and exceeded its design goals. The design luminosity was $3 \times 10^{33} \mathrm{~cm}^{-2} \mathrm{~s}^{-1}$, equivalent to $260 \mathrm{pb}^{-1}$ per day. The daily delivered and recorded integrated luminosity since October 1999 can be seen in Figure 2.2.

\subsection{The $B_{A} B_{A R}$ Detector Overview}

The BABAR detector consists of five distinct components: the silicon vertex tracker (SVT), the drift chamber (DCH), the detector of internally reflected Cherenkov light 


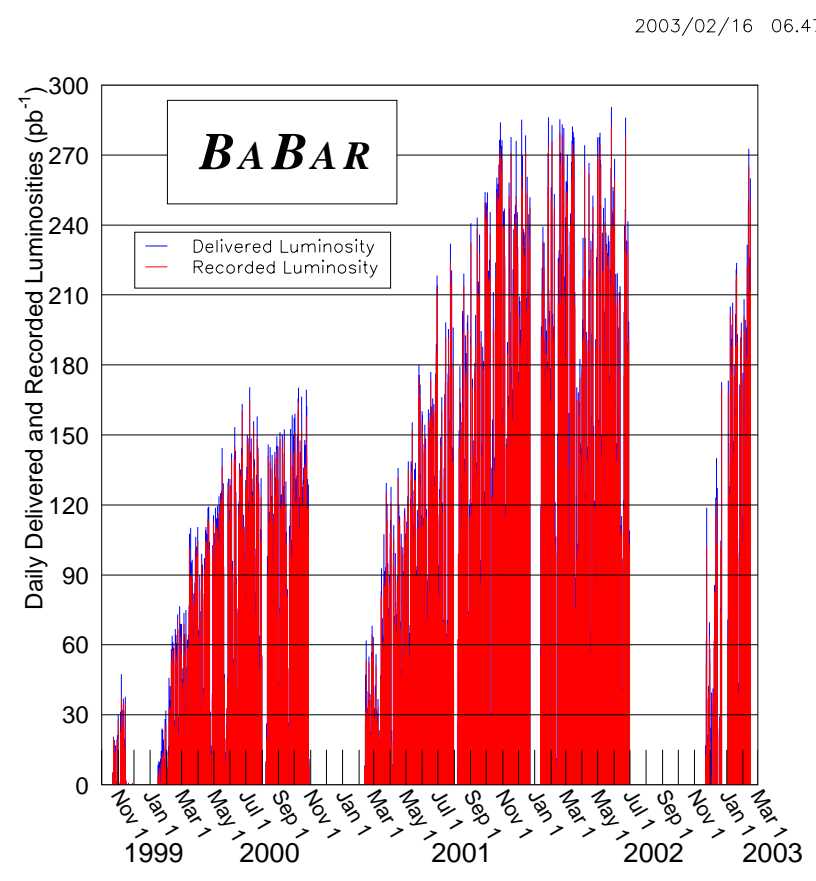

Figure 2.2: BABAR Integrated daily luminosity.

(DIRC), the electromagnetic calorimeter (EMC), and the instrumented flux return (IFR). Figure 2.3 is a longitudinal section diagram of $B A B A R$ showing the five subdetectors and the superconducting coil that encloses the tracking system. The orientation of the BABAR coordinate system is also shown. A detailed description of the detector can be found in [26].

The physics goals place several requirements on the design of the detector. To maximise coverage of collision products, there must be a large acceptance down to small angles with respect to the boost direction. To observe small signals from a broad range of physics processes, excellent reconstruction efficiency of charged and neutral particles is needed, with very good momentum, energy, and angular resolution. Effective identification is required for a wide range of particles (electron, muons, kaons, pions, and protons), in order to suppress background when reconstructing decays, 


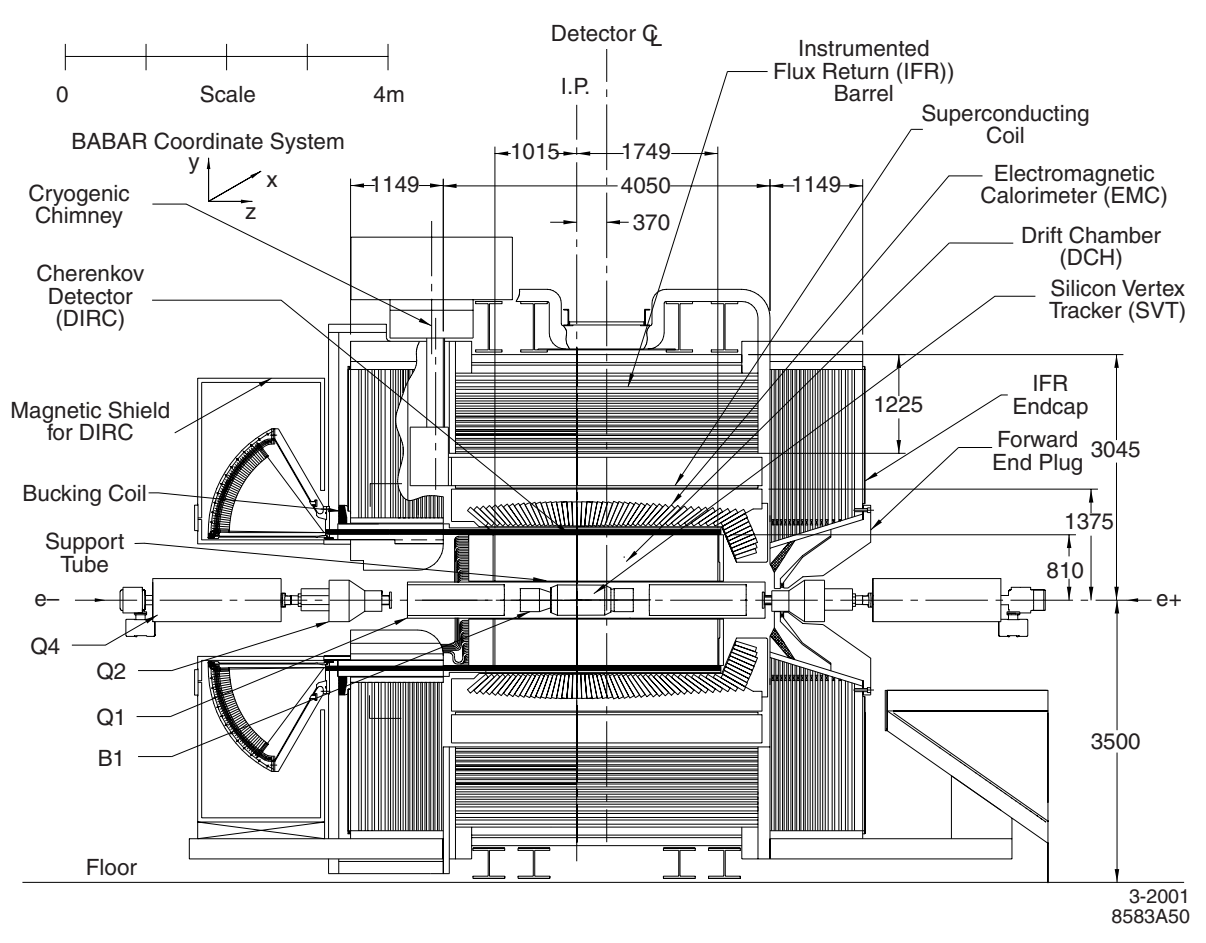

Figure 2.3: The BABAR Detector.

and to provide flavour tagging of $B$ mesons, which is crucial in the measurement of $C P$ asymmetries.

A data acquisition system and computing model, including a flexible and redundant trigger, is required to cope with the large incoming stream of data. All detector components must withstand the amount of radiation damage projected over several years of near continuous PEP-II running.

\subsection{The Silicon Vertex Tracker}

The SVT is at the heart of the central tracking system, and its purpose is to measure decay vertex positions of $B$ mesons. This enables measurement of time-dependent $C P$ asymmetries by utilising the decay time differences between $B \bar{B}$ pairs from 
$\Upsilon(4 S)$ decays. The SVT also provides reconstruction of low momentum tracks (transverse momentum $\lesssim 100 \mathrm{MeV} / c$ ) and so allows full reconstruction of manybody decay processes. Additionally, the SVT measures track $\mathrm{dE} / \mathrm{d} x$, which is used by particle identification algorithms.

The SVT sensing devices are silicon strip detectors, arranged in five concentric cylindrical layers as shown in Figure 2.4, which displays the sensor positions in the $x, y$ plane. For maximum polar angle coverage the outer two layers have angled sections at each end of the detector; the acceptance of the active volume is $21.0^{\circ}$ $\leq \theta \leq 150.1^{\circ}$ in the laboratory frame $(\theta$ is defined as the angle with respect to the $+z$ axis $)$.

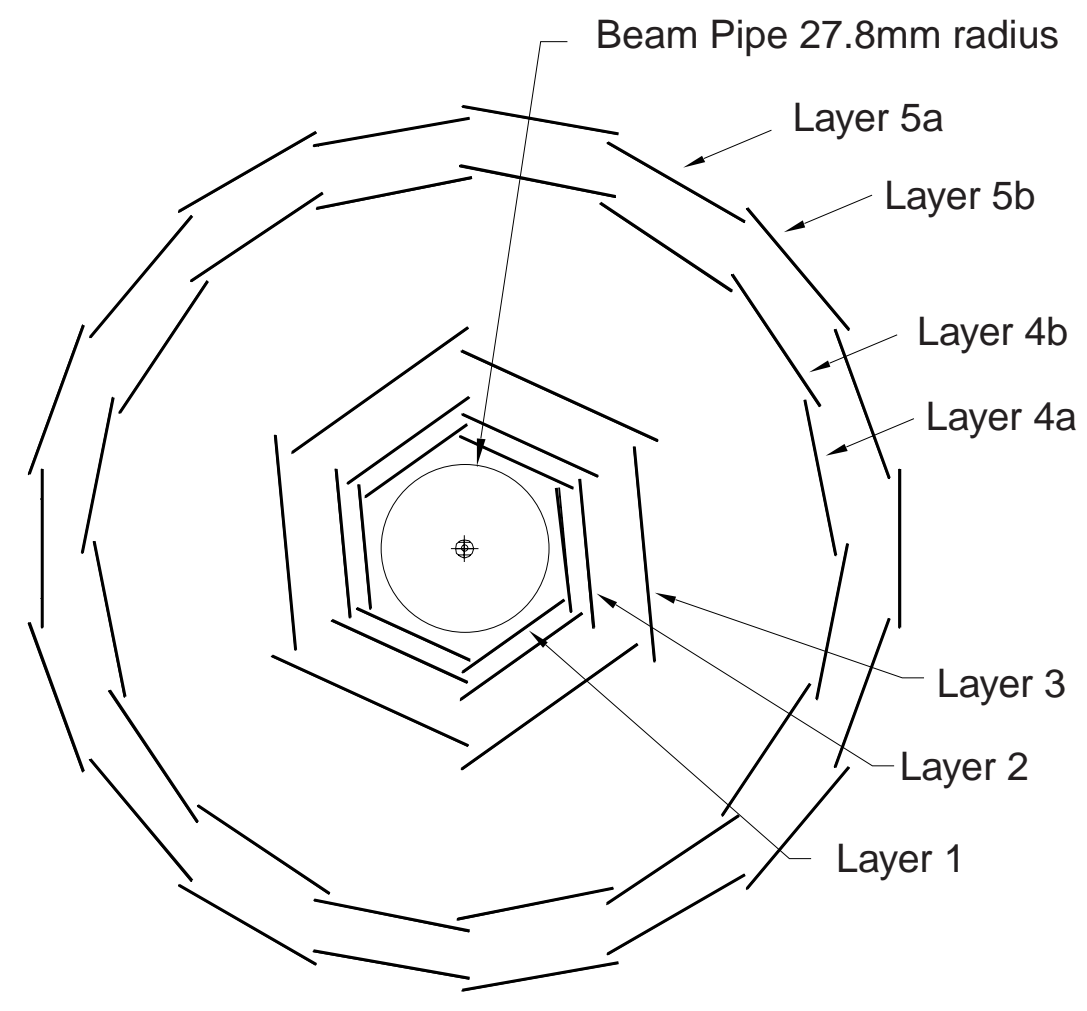

Figure 2.4: SVT transverse section diagram. The inner three detector layers are tilted by $5^{\circ}$ in $\phi$ to allow overlapping and maximum azimuthal coverage. Layers 4 and 5 are arched at each end of the detector. 
The detectors are double sided, with strips providing $z$ coordinate position and $\phi$ angle of individual signals on separate sides. The readout spacing of the strips, along with their length, and the radii of the five detector layers are given in Table 2.2.

\begin{tabular}{l|c|r|r}
\hline $\begin{array}{l}\text { Layer/ } \\
\text { view }\end{array}$ & $\begin{array}{r}\text { Radius } \\
(\mathrm{mm})\end{array}$ & $\begin{array}{r}\text { Readout } \\
\text { pitch } \\
(\mu \mathrm{m})\end{array}$ & $\begin{array}{r}\text { Strip } \\
\text { length } \\
(\mathrm{mm})\end{array}$ \\
\hline $1 \mathrm{z}$ & 32 & 100 & 40 \\
$1 \phi$ & 32 & $50-100$ & 82 \\
\hline $2 \mathrm{z}$ & 40 & 100 & 48 \\
$2 \phi$ & 40 & $55-110$ & 88 \\
\hline $3 \mathrm{z}$ & 54 & 100 & 70 \\
$3 \phi$ & 54 & 110 & 128 \\
\hline $4 \mathrm{z}$ & $91-127$ & 210 & 104 \\
$4 \phi$ & $91-127$ & 100 & 224 \\
\hline $5 \mathrm{z}$ & $114-144$ & 210 & 104 \\
$5 \phi$ & $114-144$ & 100 & 265 \\
\hline
\end{tabular}

Table 2.2: Geometric parameters for each layer and readout plan of the SVT.

The radial range for layers 4 and 5 takes into account the geometry of the angled sections.

The readout electronics of the SVT are located outside the active area of detection, and connected to the z-strips by thin circuitry (fanout circuits). The front end electronics (FEE) amplify, shape, and digitise the signals, which are recorded as Time Over Threshold (TOT) pulse-widths, along with the time and strip location; the readout is governed by the level 1 trigger (See Section 2.9). The signal to noise ratio is determined to be better than 15 for minimum ionising particles. Since the SVT is the subdetector most vulnerable to radiation damage it is important that the electronics are chosen to be radiation hard, and the SVT is protected from radiation 
damage by photodiodes which will precipitate a dump of the beams in the case of intermittent or prolonged large doses.

The performance of the SVT is judged by its hit ${ }^{1}$ reconstruction efficiency and its accuracy in calculating track parameters. The single hit efficiency is calculated in $e^{+} e^{-} \rightarrow \mu^{+} \mu^{-}$events to be $97 \%$. The spatial hit resolution is determined from the residuals of the fitted track trajectory and the positions of the hits, using two prong events ( $\operatorname{such}$ as $e^{+} e^{-} \rightarrow \mu^{+} \mu^{-}$). The resolutions for the $z$ and $\phi$ strips as a function of track incident angle are shown in Figure 2.5. The resolution is better at normal incidence for the inner layers due to their smaller readout pitch. Multiple scattering leads to poorer resolution at larger track incidence angles. The $\mathrm{dE} / \mathrm{d} x$ for each SVT track is obtained from a truncated mean calculation from the several associated signals. Each $\mathrm{dE} / \mathrm{d} x$ value is calculated from the signal pulse height, which is in turn extrapolated from the pulse width (TOT) measurement. A $2 \sigma$ separation of pions and kaons can be achieved up to $500 \mathrm{MeV}$.

Alignment of the SVT is carried out using $e^{+} e^{-} \rightarrow \mu^{+} \mu^{-}$and cosmic ray events, and utilises information of the relative sensor positions taken by an optical survey during construction. Firstly local alignment of the relative positions of the silicon sensors is performed, followed by global alignment of the SVT with respect to the coordinate system defined by the DCH. Local alignment is quite stable, and since the alignment procedure is complex it is carried out on necessity (for example, after detector access). Global alignment (an example of rolling calibration) is performed every 2-3 hours.

SVT target resolutions and reconstruction efficiencies have been met. Replacement module assembly is underway, to be employed in the inevitable course of radiation damage. Currently there are no sensor failures due to radiation damage.

\footnotetext{
${ }^{1} \mathrm{~A}$ 'hit' is defined as a recorded signal above a certain charge threshold.
} 


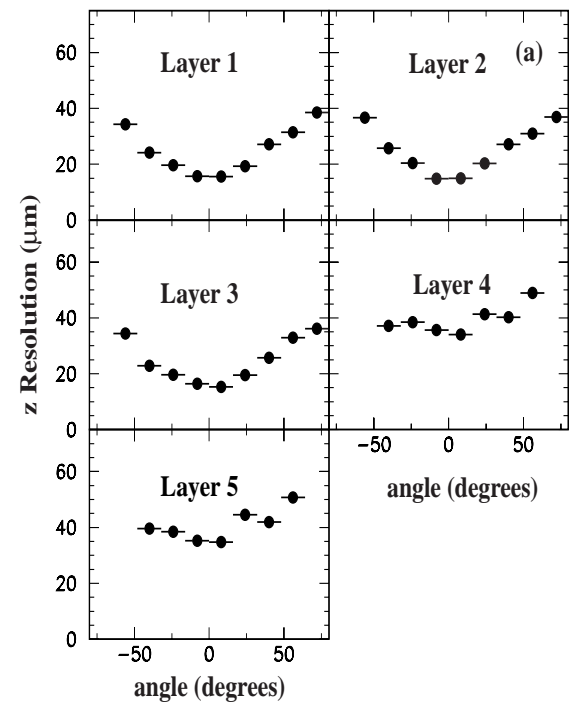

(a)

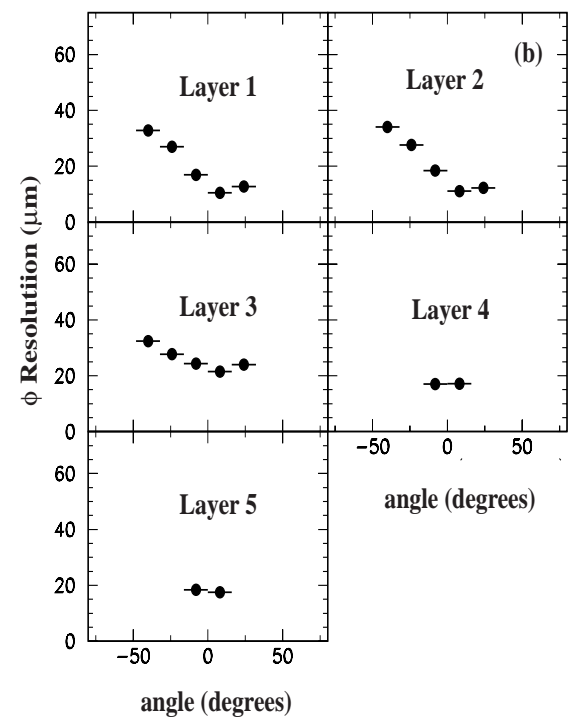

(b)

Figure 2.5: SVT hit resolution of the five sensor layers in the a) z coordinate and b) $\phi$ coordinate in microns, plotted as a function of track incident angle in degrees.

\subsection{The Drift Chamber}

Enclosing the SVT is the other part of the BABAR tracking system, the DCH. The $\mathrm{DCH}$ can be considered the main tracking device, in that reconstruction of tracks begins with information collected there.

The purpose of the DCH is primarily to provide accurate momentum measurement of charged particles. To achieve this, excellent resolution of track trajectories is required. It also provides $\mathrm{dE} / \mathrm{d} x$ measurements and so helps to identify particle types.

The DCH is a multiwire chamber that obtains spatial resolution by using the drift 
time of electrons in a gas produced by the passage of an ionising charged particle. The wires are arranged in cylindrical layers, concentric with the $z$ axis. The layers vary in radius from $260.4 \mathrm{~mm}$ to $747.2 \mathrm{~mm}$.

There are three general wire orientations, one axial (parallel to the $z$ axis) and two stereo (set at small angles with respect to the $z$ axis). The two stereo orientations provide a means of measuring the $z$ coordinates of fitted track trajectories. There are 10 superlayers, each containing 4 layers of wires, the orientation of the wires in each superlayer alternates between axial $(\mathrm{A})$ and stereo $(\mathrm{U}, \mathrm{V})$. This arrangement is shown in Figure 2.6. The length of the DCH is approximately $280 \mathrm{~cm}$, with the beam interaction point offset by $3.7 \mathrm{~cm}$ from the midpoint.

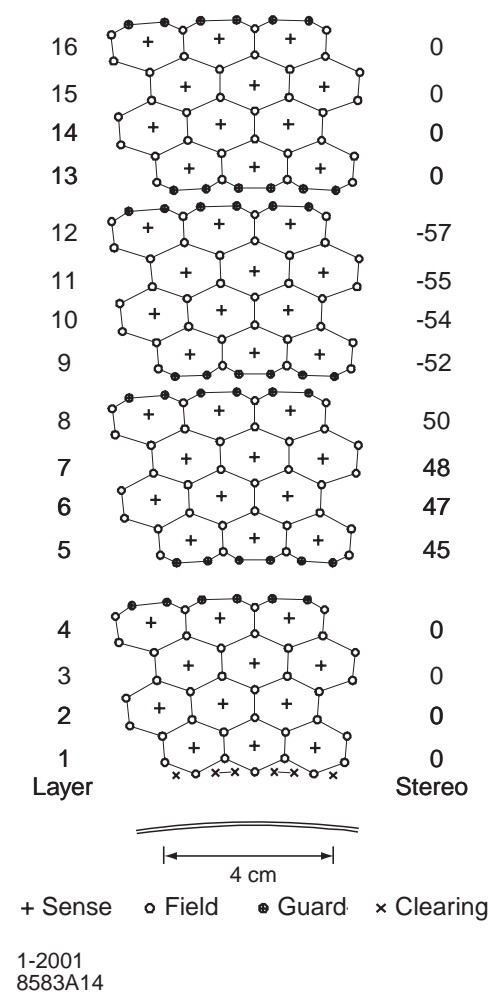

Figure 2.6: The DCH cell arrangement for the first 4 superlayers. The stereo angles of the sense wires (mrad) are given for each layer in the column labelled 'Stereo'. 
The pattern of wires in the transverse plane forms hexagonal cells, each with six sense wires surrounding a central field wire. The ionisation medium is heliumisobutane, which has a large radiation length (around $800 \mathrm{~m}$ at room temperature and atmospheric pressure). In combination with the low mass aluminium field wires and tungsten-rhenium sense wires, the material within the DCH amounts to about $0.2 \%$ of a radiation length.

Readout quantities for each wire are the time of the leading edge of the amplified signal (for drift time measurement) and summed charge (for $\mathrm{dE} / \mathrm{d} x$ measurement). Digital converters ensure the time measurements are accurate to $1 \mathrm{~ns}$. The raw digitised signals are transported to readout modules outside the detector, via optical fibres.

Drift times are converted into drift distances using a relationship derived from two prong events. The position resolution as a function of drift distance, for cells in layer 18 is shown in Figure 2.7. The $\mathrm{dE} / \mathrm{d} x$ for each track is computed as a truncated mean of the associated signals, after applying corrections for various factors which degrade the resolution of the energy loss measurement (such as effects dependent on the track entrance angle in each cell). Figure 2.8 is an illustration of the particle type discrimination achieved by the $\mathrm{dE} / \mathrm{d} x$ measurements. The $\mathrm{dE} / \mathrm{d} x$ resolution is determined to be $7.5 \%$.

The track reconstruction efficiency of the DCH is estimated using the SVT as an independent tracking device. The efficiency as a function of momentum is shown is Figure 2.9. The design operating voltage is $1960 \mathrm{~V}$, but was lowered for part of the first running phase (May 1999 to October 2000) to $1900 \mathrm{~V}$ due to damage concern. The efficiency is around 5\% lower during $1900 \mathrm{~V}$ operation. The DCH currently operates (since January 2001) at $1930 \mathrm{~V}$.

The overall resolution of reconstructed track momentum is governed by the DCH 


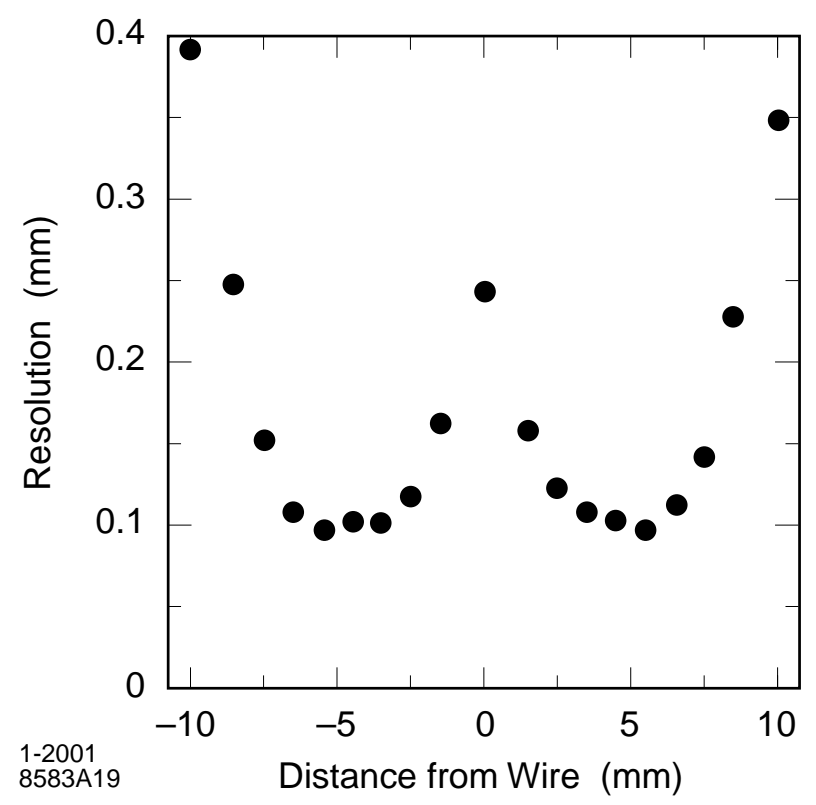

Figure 2.7: DCH position resolution.

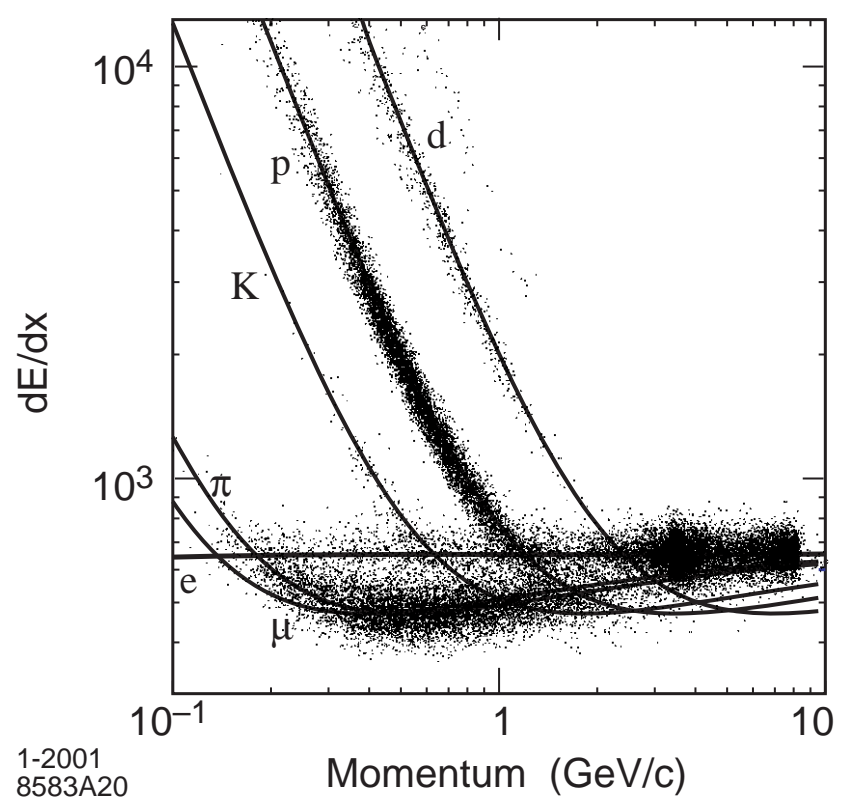

Figure 2.8: $d E / d x$ measured in the DCH, as a function of track momentum. The superimposed curves are Bethe-Bloch functions. 


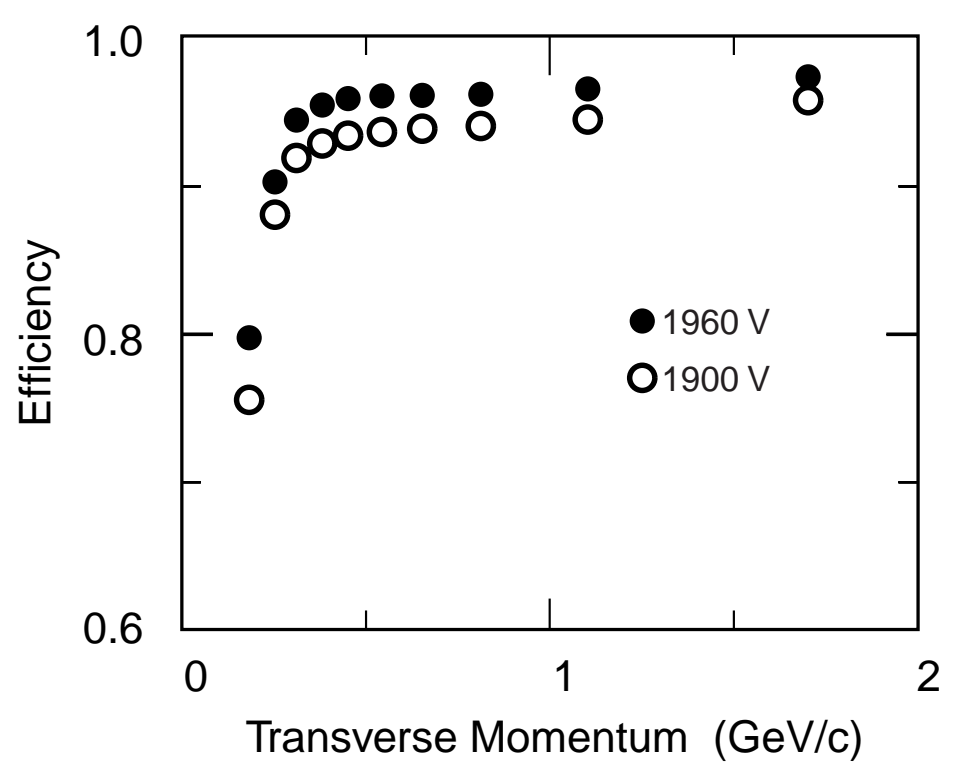

Figure 2.9: DCH track reconstruction efficiency as a function of momentum.

measurements (while the SVT determines track position and angle close to the interaction point). The transverse momentum resolution is estimated from cosmic ray muons to be

$$
\frac{\sigma_{p_{T}}}{p_{T}}=0.13 \% \cdot p_{T}+0.45 \%
$$

where the first term originates from track curvature and the second is due to intrinsic spatial detector resolution. The $p_{T}$ resolution is found to be in good agreement with the design estimates.

The resolution goals for the DCH of $140 \mu \mathrm{m}$ for track position and $7 \%$ for $\mathrm{dE} / \mathrm{d} x$ have been met. A small number of dead cells, the result of an accident during routine operations, has not noticeably affected its performance. 


\subsection{The Detector of Internally Reflected Cherenkov Light}

The ability to identify particle types is of great importance in the flavour tagging of $B$ mesons, and is extremely helpful in background suppression in decay reconstruction, for example in $B \rightarrow \eta_{c} K$ where the $\eta_{c}$ may decay to several kaons or pions. While $\mathrm{dE} / \mathrm{d} x$ measurements in the DCH and SVT partly provide particle identification, their effectiveness falls off at increasing momentum $(>1 \mathrm{GeV} / c)$. A novel type of detector, the DIRC, whose main purpose is particle identification, is employed by $B A B A R$. It uses the direct relation between Cherenkov radiation angle and the particle speed to distinguish different types of particle.

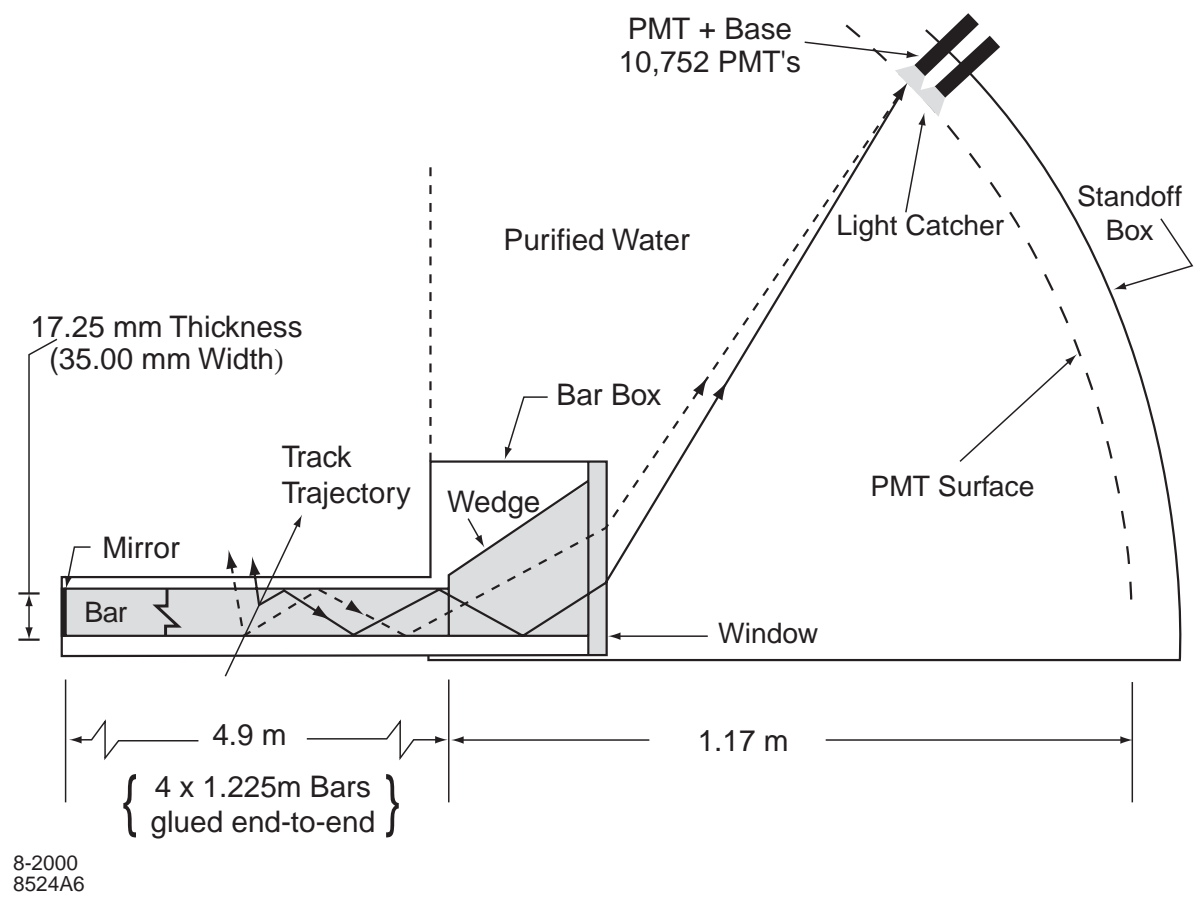

Figure 2.10: Schematic of light production, transportation and detection in the DIRC. The wedge has a slightly angled lower edge to minimise displacement of the folded half of the image from the unfolded half. 
The DIRC is a 12-sided barrel structure of approximately $5 \mathrm{~m}$ long silica bars of rectangular cross-section. Cherenkov light of traversing particles is transported by total internal reflection along the bars, which preserve the angle of light emission. At one end of the bar the light is expanded into a volume of purified water before being collected by an array of photomultiplier tubes (PMTs). A mirror is placed at the other end of the bar, to reflect light back to the instrumented end. The process of light emission, transportation and detection is shown in Figure 2.10 which also denotes the dimensions of the bars and position of the PMTs. A trapezoidal wedge attached to the end of each bar reflects light at large angles back onto the detection arrays, and folds one half of the Cherenkov image onto the other half.

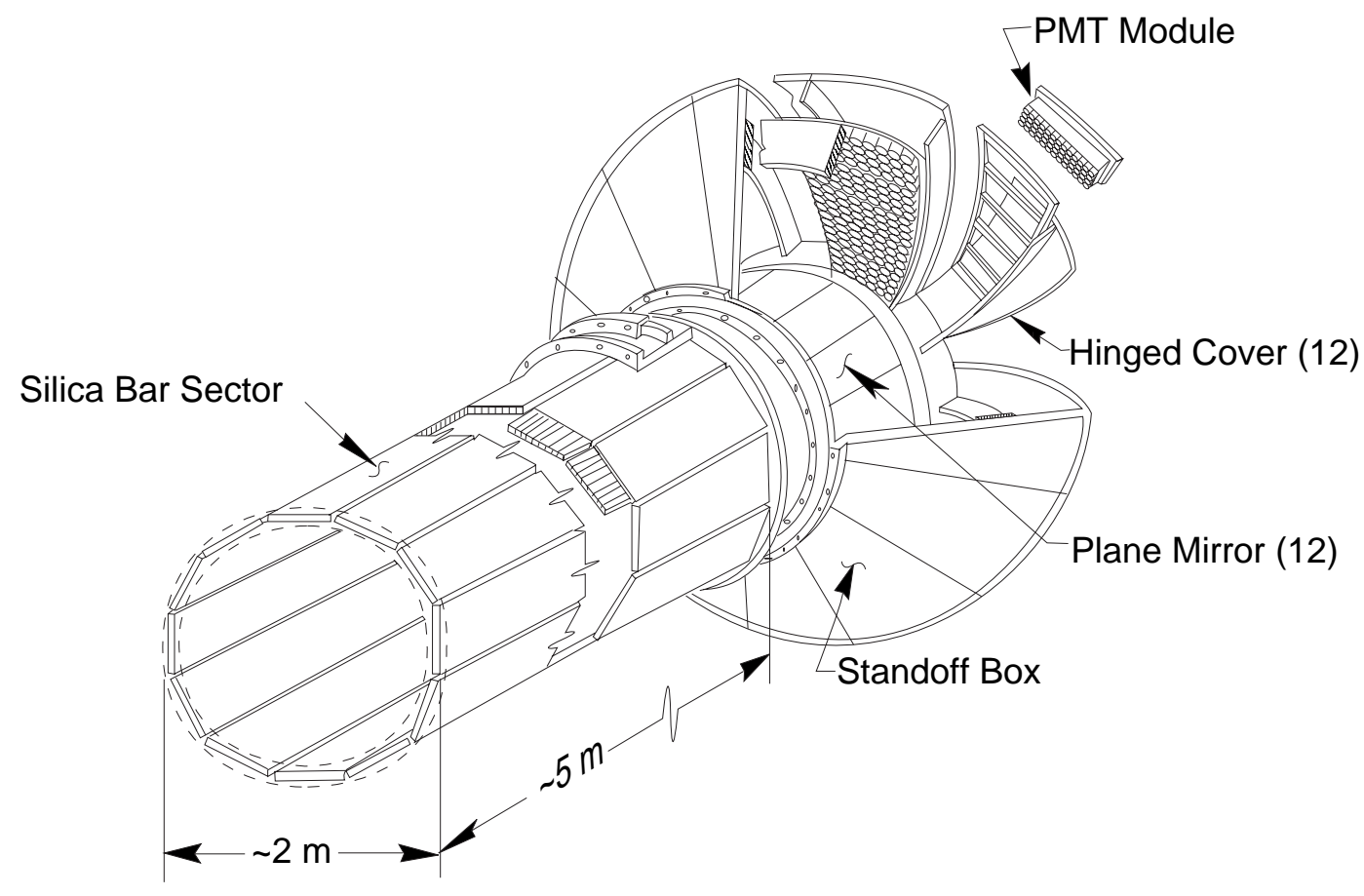

Figure 2.11: Mechanical view of the DIRC components.

Each of the 12 sides of the DIRC barrel consists of a bar box containing 12 optically isolated bars, and the whole structure is enclosed in an aluminium support tube which is anchored to the body of the IFR. The water into which the light expands is contained in a cone-shaped box (standoff box), which contains the 12 sectors 
of PMTs. Each detection sector contains 896 PMTs, each $29 \mathrm{~mm}$ in diameter and equipped with light concentrators. The PMTs are protected from the BABAR magnetic field by a steel shield. The whole arrangement can be seen in Figure 2.11. The DIRC occupies $80 \mathrm{~mm}$ of radial space in the BABAR detector, corresponding to 0.17 radiation lengths at normal incidence.

Reconstruction of Cherenkov angles of tracks and calculation of the likelihoods of particle hypotheses is made possible by combining the position and time information of the PMT signals. The accuracy of the track Cherenkov angle is limited by photon angular resolution, the number of photons detected, the timing resolution and the granularity of the PMTs. The photon yield depends on the track incident angle, being lowest for tracks at normal incidence, but is always high enough to ensure a Cherenkov angle resolution sufficient to separate pions and kaons with momenta up to $4 \mathrm{GeV} / c$. This is illustrated in Figure 2.12 where the separation is calculated for kaons from $D^{0} \rightarrow K^{+} \pi^{-}$decays from inclusive $D^{*}$ production. Shown also is the Cherenkov angle for selected 'kaons' from this sample.

The DIRC functions well as a particle identification device and provides the required level of kaon-pion separation. Radiation damage is not a major concern, since the silica bars' performance was found to be only negligibly affected during tests with doses of $100 \mathrm{kRad}$.

\subsection{The Electromagnetic Calorimeter}

Many decays among those seen at $B A B A R$ include one or more $\pi^{0}$ s, for example $B \rightarrow \eta_{c} K, \eta_{c} \rightarrow K^{+} K^{-} \pi^{0}$. Processes involving an $\eta$ in the decay tree are also prevalent. The BABAR EMC allows the reconstruction of such particles which decay to two photons. For the detection of photons to be useful, excellent energy and 


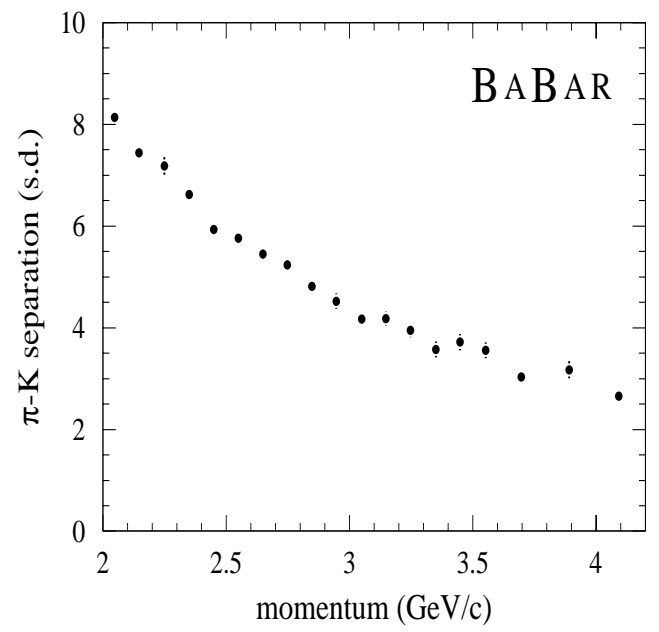

(a)

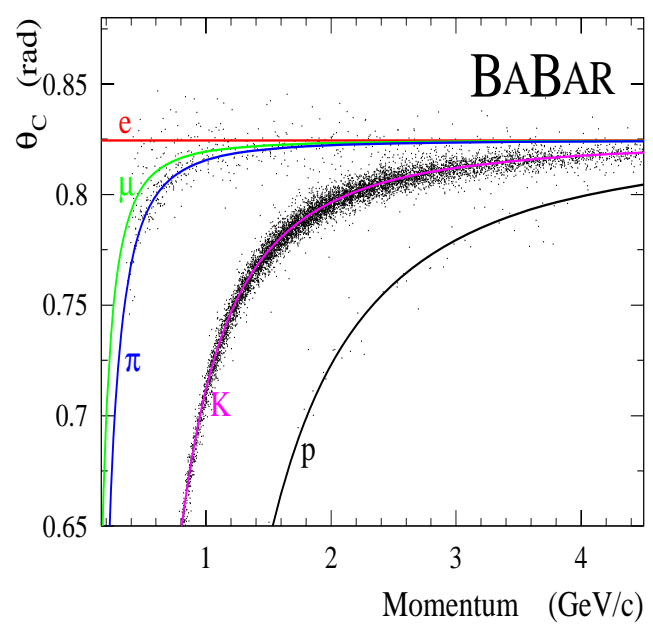

(b)

Figure 2.12: DIRC performance measured from $D^{*}$ control sample a) $K \pi$ separation b) Cherenkov angle for selected 'kaons', which includes background from other particle types.

position resolution is required. Another essential service of the EMC is to provide electron identification, for $B$ flavour tagging.

The EMC consists of a highly segmented array of CsI crystals, in which electromagnetic showers yield the position and energy of incident photons and electrons. The crystals are arranged in a barrel of approximately $1 \mathrm{~m}$ in radius with an endcap in the direction of the high energy electron beam. The polar angle coverage is $15.8^{\circ}$ $\leq \theta \leq 141.8^{\circ} .48$ rings of 120 crystals form the barrel component of the EMC, while the endcap contains 8 rings. A side view of this arrangement is shown in Figure 2.13 .

CsI has several properties which make it a suitable choice of scintillation material; its small radiation length and Moliere radius provide shower containment, while its high light yield ensures good energy resolution and allows the use of silicon photodiodes. 


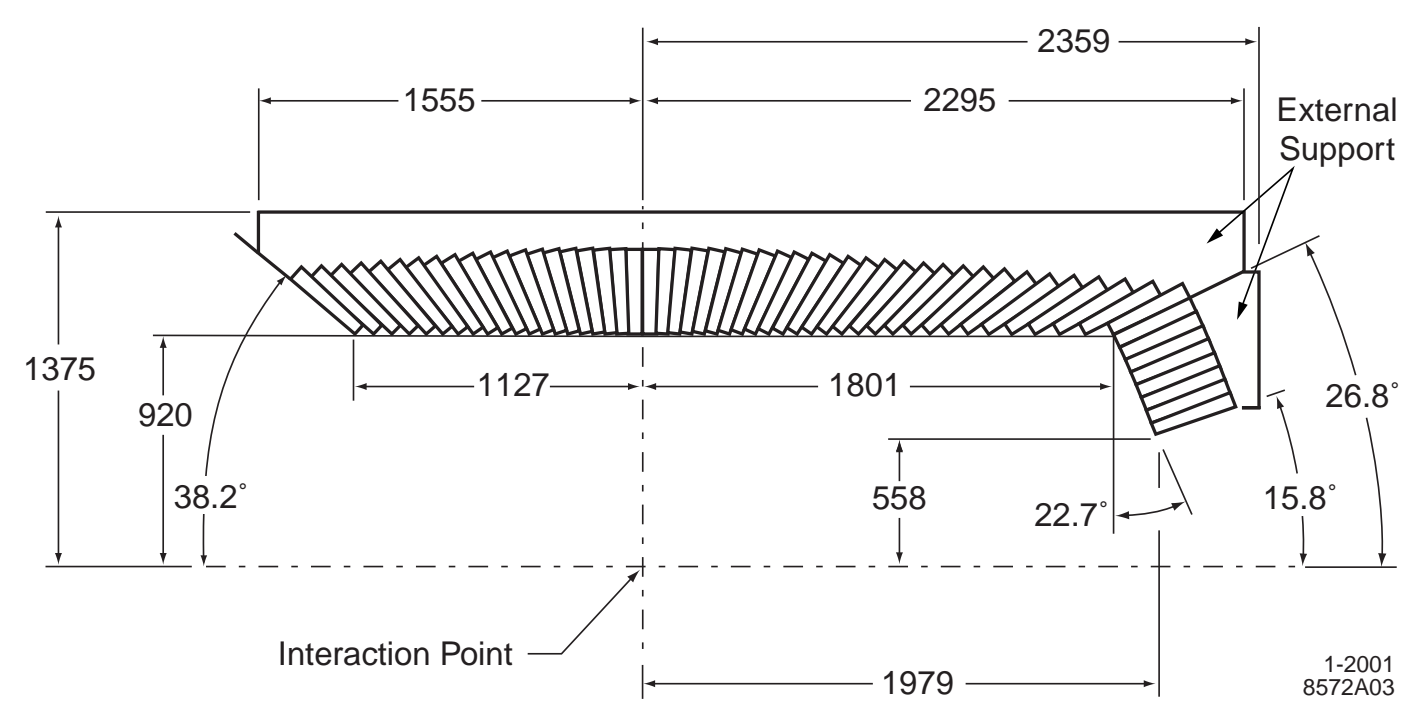

Figure 2.13: Longitudinal section diagram of the EMC.

The crystals are trapezoidal and vary in length over the polar angle range, with the longest in the forward direction. The crystal axes do not point directly at the $e^{+} e^{-}$ interaction point, so as not to lose particles in the gaps between the crystals. Each crystal is polished and wrapped with reflective material to minimise leakage.

The light yield in each crystal is read out by two photodiodes. Preliminary amplification and shaping of the signal is performed at the crystal, followed by further amplification and digitisation outside the body of the detector. Upon receiving a trigger signal, samples within a $1 \mu$ s time interval are readout for feature extraction.

Several levels of calibration are applied to the EMC signal reconstruction chain. These include electronics calibration, determination of single crystal energy scale, and corrections for shower leakage. The calibration techniques are described in more detail in Chapter 3.

The reconstruction of energy deposits in the EMC involves the formation of clusters of adjacent crystal signals. The clustering procedure is described in Section 2.10. Track-cluster matching is used to associate showers with charged particles passing 
through the tracking system; 15.8 clusters are detected in an average hadronic event, with 10.2 not matched to any track.

The energy resolution of the EMC is measured by several processes including $\pi^{0}$ and $\eta$ decays, Bhabha ( $e^{+} e^{-}$scattering), and radiative charmonium transitions; see Figure 2.14. A fit to the energy dependence of the resolution yields

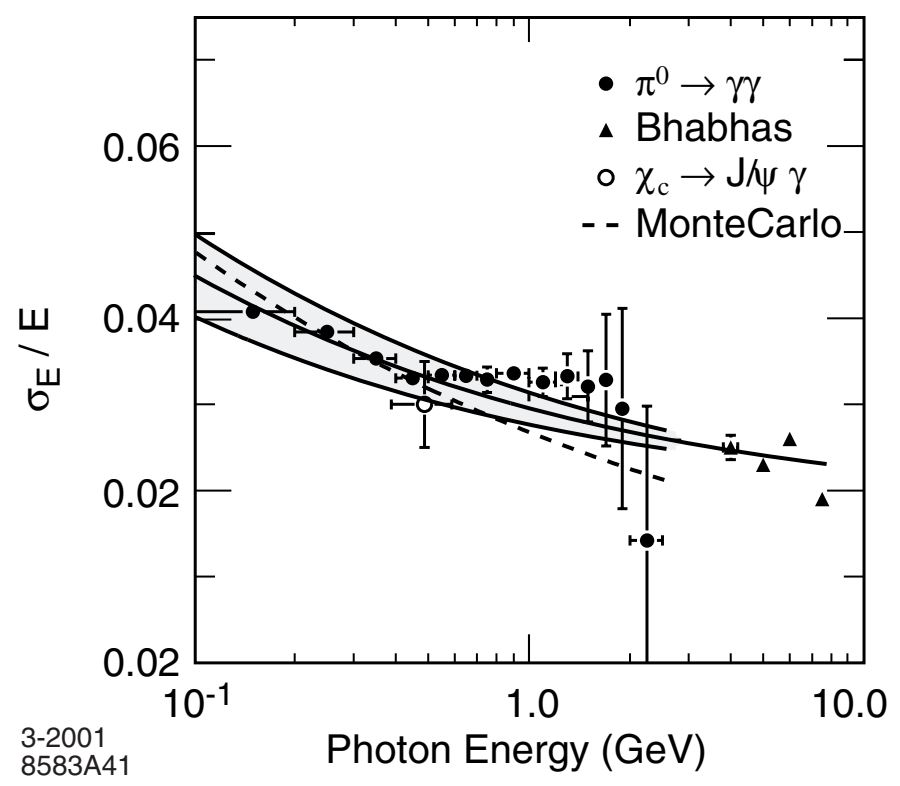

Figure 2.14: EMC energy resolution from various processes. The solid lines are the central and $\pm 1 \sigma$ values of the fit function (see text).

$$
\frac{\sigma_{E}}{E}=\frac{2.32 \%}{\sqrt[4]{E(\mathrm{GeV})}} \oplus 1.85 \%
$$

where the first component is statistical, and the second arises from systematic effects such as shower leakage, absorption, or calibration inaccuracies. Similarly, the position resolution is inferred from $\pi^{0}$ decays and found to be $\sigma_{\theta}=3.87 \mathrm{mrad} / \sqrt{\mathrm{E}(\mathrm{GeV})}$. The $\pi^{0}$ mass resolution is measured to be $6.9 \mathrm{MeV} / c^{2}$ in $B \bar{B}$ events, agreeing well with Monte Carlo predictions. As an electron identifier, using primarily $E / p$ measurements, the EMC performs with high efficiency, ( 90\%, for Bhabha events at the most demanding identification level). 
Currently, the EMC performance is close to the level set out in the design specifications. In the future, radiation damage and increased luminosity may affect the crystal response and background handling.

\subsection{The Superconducting Coil}

The 1.5 T magnetic field which allows momentum measurement of charged particles is provided by a superconducting solenoid positioned between the EMC and the IFR.

The solenoid is a double layered coil kept at low temperature $(4.5 \mathrm{~K})$ by circulating liquid helium within its support structure. The support structure is an aluminium cylinder of central radius $1.53 \mathrm{~m}$.

The field is required to have a minimal transverse component near the beam-focusing quadrupole magnets, to avoid demagnetisation. Flux leakage is also a concern, particularly at the back of the detector, near the PMT array of the DIRC. To circumvent this, a reverse field is applied by a bucking coil situated next to the DIRC standoff box.

A complete 3-dimensional map of the field strength is required for track reconstruction. Hall probes attached to a rotating, gliding propeller measured the magnetic field at many $z, \phi, r$ positions. The final field is parametrized by a polynomial fit to these measurements. The fit includes terms to describe the perturbations caused by the dipole and quadrupole magnets. 


\subsection{The Instrumented Flux Return}

The largest BABAR detector component is the IFR. Its purpose is dual, serving as a return yoke for the flux of the magnetic field, and as a detector of muons and $K_{L}^{0} \mathrm{~S}$. These functions are particularly important for $C P$ violation measurements, which rely on the reconstruction of $B^{0} \rightarrow J / \psi K_{S}^{0}$ and $B^{0} \rightarrow J / \psi K_{L}^{0}$ where the $J / \psi \rightarrow \mu^{+} \mu^{-}$is a major reconstruction mode. Muons are not easily distinguishable by the other subdetectors.

The active components of the IFR are Resistive Plate Chambers (RPCs), which operate on the principle of streamer emission from ionising particles. A cross-section of a typical RPC module is shown in Figure 2.15.

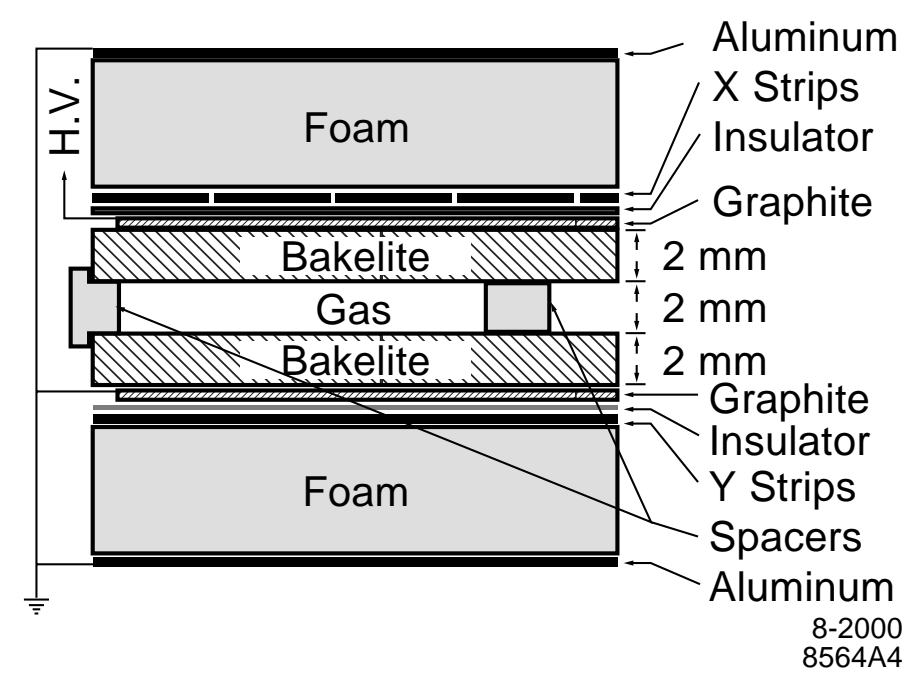

Figure 2.15: IFR RPC longitudinal cross-section diagram.

The RPCs are interleaved between the steel sheets of the flux return, which are arranged in a $\sim 4 \mathrm{~m}$ long hexagonal barrel and two end doors. There are 19 layers of RPCs in the barrel and 18 in the end doors. The steel sheets vary in thickness from $2 \mathrm{~cm}$ for the innermost to $10 \mathrm{~cm}$ for the outermost, giving a total thickness of $65 \mathrm{~cm}$ for the barrel walls and $60 \mathrm{~cm}$ for the doors. Between the EMC and the 
magnet there are another 2 layers of cylindrical RPCs, for the detection of particles exiting the EMC. Each RPC module maintains a high voltage $(\sim 8 \mathrm{kV})$ across a gap filled with an argon-Freon-isobutane gas mixture. The gap is enclosed by two bakelite sheets coated with linseed oil on the inner surface and graphite on the outer surface (to provide the voltage terminal). Aluminium readout strips on each side of the gap provide the signal positions in orthogonal directions.

For the barrel RPCs, the strips measure the $z, \phi$ coordinates; the strip pitch varies from $19.7-32.8 \mathrm{~mm}$ in the $\phi$ direction and is constant at $38.5 \mathrm{~mm}$ in $z$. For the RPCs in the doors the $x, y$ positions are measured with strip pitches of $38.0 \mathrm{~mm}$ and 28.3 $\mathrm{mm}$ respectively. The multiple layers enable 3-dimensional reconstruction of IFR clusters.

For the identification of muons, tracks are extrapolated into the IFR and associated with the reconstructed clusters found there. The discriminating quantities are the interaction length, cluster distribution amongst strips, and track-cluster residuals. The efficiency and misidentification levels are measured on $e^{+} e^{-} \mu^{+} \mu^{-}$final states and $K_{S}^{0} \rightarrow \pi^{+} \pi^{-}$decays, yielding $90 \%$ efficiency for muons with $6-8 \%$ pion misidentification probability. $K_{L}^{0} \mathrm{~S}$ are identified by clusters not associated with any extrapolated track, and are detected with an efficiency increasing linearly from $20 \%$ to $40 \%$ in the momentum range $1 \mathrm{GeV} / c$ to $4 \mathrm{GeV} / c$.

Although the IFR satisfies its purpose as an effective muon identification system, the efficiency of a large number of the RPCs has been falling since the first year of operation. This is thought to be the effect of electrical shorting by linseed oil filaments, formed in high temperature conditions. The projected threat to performance is serious and replacement of all RPCs is scheduled by 2005 . 


\subsection{The Trigger}

The aim of the BABAR trigger is to control the readout of detector information for interesting events, distinguishing them from the ever-present levels of background and noise that give rise to signals in various parts of the detector. The data acquisition system can cope with a limit of $2.5 \mathrm{kHz}$ input, and it is part of the trigger's function to reduce the data flow rate below this level. The BABAR trigger system consists of the level 1 (L1) and level 3 (L3) triggers. The L1 trigger is implemented in the hardware, while the L3 trigger is purely software. There is no level 2 trigger.

\subsubsection{The Level 1 Trigger}

The L1 trigger components are the drift chamber trigger, (DCT), the electromagnetic calorimeter trigger (EMT), and the instrumented flux return trigger (IFT) which enable fast identification of charged tracks, electromagnetic showers, and $\mu^{+} \mu^{-}$events/cosmic rays respectively. The global trigger (GLT) processes information in the form of trigger primitives from the EMT, DCT, and IFT to form specific triggers. The primitives are then passed to the fast control and timing system (FCTS), which can impose the trigger configuration, and issues a readout command (L1 Accept) if the event is of interest. The time delay of the whole L1 process is about $11 \mu \mathrm{s}$, just within the buffer capacity $(12.8 \mu \mathrm{s})$ of the front end electronics.

Using a crude track segment finding and linking routine, the DCT determines the existence of tracks at two different length thresholds, those crossing the whole and half of the DCH superlayers. Tracks with a minimum value of transverse momentum are also selected by a $p_{T}$ discriminator. The EMT indicates energy deposits in groups of crystals (towers) within the EMC barrel and endcap. Energy is summed 
in separate $\phi$ regions and compared to a set number of thresholds to provide the EMT primitives. The IFT output is a trigger word encoding several trigger conditions, which include detection of muons, singly or in pairs, encompassing all $\mu^{+} \mu^{-}$events of interest.

Trigger lines are the result of logical operations performed by the GLT on the primitives supplied by the three L1 components. The trigger time is estimated from the highest priority line and other triggers with inconsistent times are rejected. Finally event readout is authorised by the FCTS. The combined L1 trigger efficiency for hadronic $B$ events is estimated to be above $99.9 \%$. Background processes dominate the typical operating rate of $1 \mathrm{kHz}$, while $e^{+} e^{-}$scattering and annihilation accounts for $\sim 130 \mathrm{~Hz}$.

\subsubsection{The Level 3 trigger}

The software (L3) trigger has access to the full event readout as well as the L1 output. It refines the measurement of tracks and clusters before classifying whole events and writing information to files for further processing and reconstruction. The output rate of the L3 trigger matches the frequency of occurrence of useful physics events, around $100 \mathrm{~Hz}$.

The L3 track finding sequence provides three-dimensional track trajectories in the DCH, with time information and sufficient resolution to reject background tracks originating from close to the interaction point. Clustering of the EMC crystal signals rejects low energy and out-of-time deposits due to noise and background, which can dominate the occupancy of the EMC readout. Filters then select events with track and cluster quality criteria (such as minimum value of $p_{T}$, closest approach to beam spot, energy) imposing a veto to reduce the very high rate of Bhabha scattering. Certain types of event are marked out for use by calibration routines, including 
Bhabha and radiative-Bhabha events.

The efficiency of the L3 trigger is determined from Monte Carlo to be above $99.9 \%$ for $B \bar{B}$ events. Half of the output rate at current luminosity values comprises important physics processes (with background contamination), while the remainder consists of calibration samples.

\subsection{Data Acquisition and Event Reconstruction}

The conversion of raw data from the detector subcomponents to a form that includes all necessary quantities on which to perform a physics analysis involves several stages.

\subsubsection{Data Acquisition}

The basic data acquisition (DAQ) process is as follows. The L1 trigger as described above generates event readout likely to hold physics content; the L1 Accepts form the fundamental sample of event candidates. Online event processing (OEP), which includes the L3 trigger, classifies events from the L1 sample and rejects background, vastly reducing the amount of data to be reconstructed. The output of this is a file which is fed to the online prompt reconstruction (OPR) system. Colliding-beams data is collected in numbered runs - periods (typically one or two hours long) in which the $e^{+} e^{-}$beams collide at the required luminosity. 


\subsubsection{Online Reconstruction}

OPR creates the fundamental lists of physics objects in each event required for particle decay reconstruction. These include basic tracks and clusters, Cherenkov angles, particle identification hypotheses, and muon hits.

The tracking algorithms are based on signals in the DCH and SVT, beginning with the DCH superlayer segments used in the L3 routines, and work iteratively to improve track spatial resolutions. At each iteration the event start time estimate improves and leads to a better track fit on the subsequent iteration. Tracks are then extended into the SVT and hits compatible in space and time are added to the existing track trajectory. The whole track is then refitted using the Kalman filter method [27], which takes into account detector materials and ensures the correct error calculation on the track parameters. Remaining unlinked hits in the SVT are then fit independently. The final resolution of track momentum in the DCH is given in Section 2.4. The output of the track fitting routine is a list of tracks, each with a set measured of measured parameters, called the ChargedTracks list, which is then accessible for physics analyses using the BABAR software.

The EMC cluster formation requires a single crystal with a measured energy above $10 \mathrm{MeV}$ and neighbouring crystals above a lower threshold $(1 \mathrm{MeV})$. A cluster can contain more than one energy maximum, in which case is divided into two or more bumps. The positions of bumps and clusters are determined through a energyweighted average of the geometric centres of the individual crystals. Track-cluster matching distinguishes neutral clusters (photons, $\pi^{0} \mathrm{~s}$ ) from those due to charged particles. The output list of photon candidates from EMC reconstruction is the CalorNeutral list, which contains neutral bumps not associated with any track. Merged $\pi^{0}$ candidates, in which in two bumps are not discernible within a cluster are collected in the PiOMergedDefault list. 
The DIRC uses the time and positions of PMT signals to reconstruct track Cherenkov angles. In addition, likelihoods are computed for various particle hypotheses for each track. More elaborate selection algorithms combine DIRC likelihoods with SVT and DCH measurements, and provide different levels of discrimination for each particle type.

3-dimensional clusters in the IFR are built and matched to tracks to provide muon identification as described in Section 2.8.

Several online calibrations that require physics events are performed in OPR. The EMC cluster energy calibration using $\pi^{0}$ events is an example of a rolling calibration and is described in Chapter 3.

Events with characteristic signatures are assigned tagbits. As well as simple classifications for multihadronic, radiative Bhabha, $e^{+} e^{-} \rightarrow \mu^{+} \mu^{-}$events, there are tagbits denoting more specific event content, such as the presence of $B \rightarrow \eta_{c} K$ decays. The tagbits for each event are stored in the database as logical variables. They enable a more efficient reconstruction of final physics processes; by first checking the tagbit, only events with interesting signatures can be reconstructed.

The basic lists of candidate particles described above are permanently stored in a database and can be accessed through protocols defined by the BABAR software framework. Currently, composite candidates are not stored, but must be constructed by the user's analysis application. Many software modules exist to build basic candidates such as $K_{S}^{0}, \pi^{0}$ on an event-by-event basis. Due to the flexibility of the object-orientated reconstruction sequence, it is relatively simple to define a new application to reconstruct more complicated composites, for example, a particular $B$ decay. A typical analysis program will include all those modules required to reconstruct each particle in the decay tree, applying vertex and mass constraints, and other selections where desired. Tools have also been developed to produce tra- 
ditional files of data (ntuples) for interactive tuning of selections and full analysis studies. The $B \rightarrow \eta_{c} K$ analysis described in Chapters 4 and 5 makes full use of the $B A B A R$ software functionality to provide its basic datasets.

\subsubsection{Reprocessing}

As reconstruction and calibration algorithms improve over the lifetime of the BABAR experiment, it becomes desirable to repeat the reconstruction procedure to improve the accuracy and reliability of physics analyses. For example, improvements in the alignment algorithms of the tracking system and DIRC and have lead to greater reconstruction efficiency of $C P$ eigenstates. The addition of improvements to the OPR algorithms (usually during a shutdown period) is followed by reprocessing of the data that was taken before the improvements were installed. Meanwhile, data continues to be accumulated and processed with the improved OPR code.

There have been two generations of reconstruction code in BABAR data taking. A third generation of reconstruction algorithms is currently being used to reconstruct data taken from the beginning of the BABAR lifetime. 


\section{Calibration of the Electromagnetic Calorimeter with $\pi^{0} \rightarrow \gamma \gamma$ Events}

The electromagnetic calorimeter (EMC) of the BABAR detector must be calibrated to ensure the correct energy measurements of incident photons and electrons. Reconstruction of $B$ mesons or other states decaying into one or more $\pi^{0} \mathrm{~s}$ (for example the $B \rightarrow \eta_{c} K, \eta_{c} \rightarrow K^{+} K^{-} \pi^{0}$ channel studied in Chapters 4 and 5) depends on accurate measurement of the photon energies. A method of photon energy calibration using constraints from the decay $\pi^{0} \rightarrow \gamma \gamma$ has been developed and is described here. 


\subsection{EMC Calibration}

The relation between the initial signal caused by an energy deposition in the calorimeter and the estimated true energy of that deposition is a sequence of several different calibrations. The response of the electronics, single crystal light yield and effect of shower loss in cluster reconstruction must all be calibrated. For a general description of the EMC calibration methods, see [28].

\subsubsection{Electronics Calibration}

The electronics calibration consists of pedestal subtraction and a precision charge injection procedure to linearise the response of the readout chain including photodiodes and amplification devices [29]. Cross-talk is a problem for neighbouring electronics channels (6 MeV cross-talk for a $1 \mathrm{GeV}$ incident particle [26]) and an energy dependent correction is applied to counter this effect.

\subsubsection{Single Crystal Calibration}

The energy relation to signal response for each of the 6,580 crystals is determined at two points covering the whole physical energy range of incident particles at $B A B A R$ energies.

The low energy point is provided by a radioactive source calibration, which uses photons of precisely known energy $(6.13 \mathrm{MeV})$ from the cascade decay of radioactive fluorine. The fluorine is passed through fine tubes than run along the front faces of the crystals. This calibration is performed about once a week.

At the high end of the energy scale Bhabha events provide the calibration, where 
electrons and positrons energies range from $3 \mathrm{GeV}$ to $9 \mathrm{GeV}$. The high rate of Bhabha events allows for rapid calculation of calibration coefficients for all the crystals. The method used to extract the crystal coefficients $c_{i}$ is the minimisation of the chisquared quantity [28]

$$
\chi^{2}=\sum_{k} \frac{\left(\sum_{i} c_{i} E_{i}^{\prime}-E^{k}\right)^{2}}{\left(\sigma_{E}^{k}\right)^{2}},
$$

over $k$ electromagnetic showers, where $E_{i}^{\prime}$ is the measured 'energy' reported by the DAQ system of the the $i$ th crystal in the shower, and $E^{k}$ is the expected incident energy of the particle creating the shower. The expected energy is predicted at each polar angle by Monte Carlo simulation. $\left(\sigma_{E}^{k}\right)$ is the error on the difference between the measured and expected energy, and is dominated by the error on the measured energy. The coefficients are updated about once per month, and provide a calibration accurate to $0.35 \%$.

All single crystal energies are provided by the radioactive source calibration and the Bhabha calibration. An interpolation is performed between the two points they provide on the calibration curve. Clusters and energy depositions formed with only single crystal calibration are referred to as raw clusters and energies.

\subsubsection{Cluster Calibration}

Once the energy measurement of individual crystals is calibrated, an additional correction needs to be applied to the raw cluster energies account for shower leakage (so-called global calibration). The effect of leakage can be seen for example in the reconstructed invariant $\pi^{0} \rightarrow \gamma \gamma$ mass (see Figure 3.1), where the single crystal calibration has been applied to the photon energies. The peak of the distribution lies approximately $10 \mathrm{MeV} / \mathrm{c}^{2}$ lower than the expected value.

The photons from radiative Bhabha events [30] can be used to provide this correction 


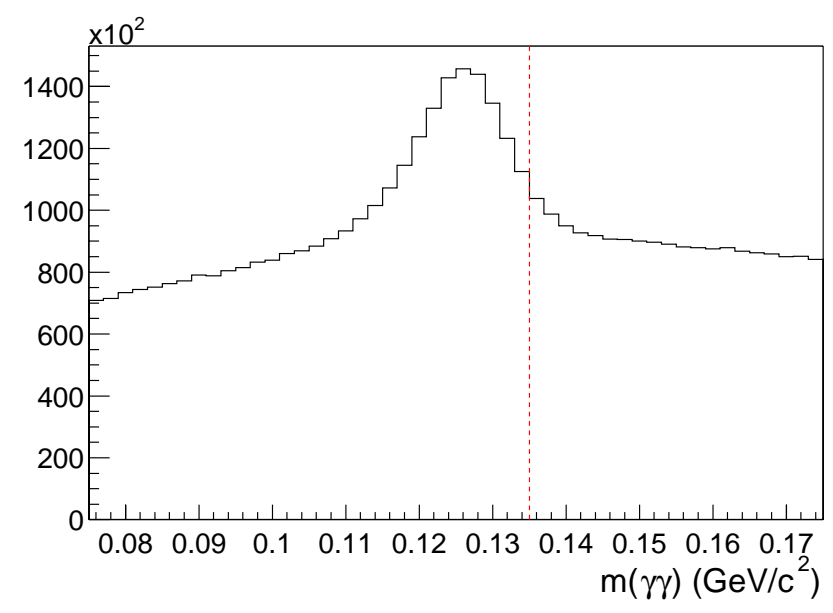

Figure 3.1: Reconstructed $\pi^{0} \rightarrow \gamma \gamma$ mass distribution with no shower leakage correction. The energy of the $\gamma \gamma$ combination is required to exceed $250 \mathrm{MeV}$. The dashed red line indicates the value of the nominal $\pi^{0}$ mass.

at the higher energies $(0.8 \mathrm{GeV}$ to $9.0 \mathrm{GeV})$. The expected energy of a photon can be extracted from a kinematic fit using the initial and final values of the scattered electron and positron momenta and the angular position of the photon. The ratio of the expected to measured energies $\left(E_{\text {meas }} / E_{\text {exp }}\right)$ as a function of energy and polar angle is then used to determine a calibration function. In practice, the behaviour of $E_{\text {meas }} / E_{\text {exp }}$ is complicated, and various ways to parametrize the calibration function have been investigated, including interpolations from bin to bin in energy. Currently the high energy cluster calibration is derived from single photon Monte Carlo, to be replaced by radiative Bhabha calibration from colliding beam events eventually.

At lower energies the constraint imposed on the photon energy by $\pi^{0} \rightarrow \gamma \gamma$ events can be used to provide a shower leakage correction. The aim is to provide a calibration function that is effective down to the lowest reconstruction energy of photons, $30 \mathrm{MeV}$. A method to provide this calibration is described in the next sections. 


\subsection{Overview of $\pi^{0}$ Calibration Method}

A correction to the measured photon energy is sought such that the reconstructed $\pi^{0}$ mass distribution matches the expected one; the corrected peak position should be equal to the nominal (world average [5]) value for the $\pi^{0}$ mass. The general method of calibration using $\pi^{0} \rightarrow \gamma \gamma$ events has been employed before by other experiments using a CsI electromagnetic calorimeter [31].

The aim is to provide a run-by-run shower leakage rolling calibration. There are several reasons why the global calibration correction may need to be updated on a short time scale; these include temperature variations, inaccuracies in the electronics calibrations or single crystal calibrations (the various calibrations may performed at irregular intervals and not necessarily simultaneously), radiation from beam injections and dumps (see [28]). The goal of the studies presented here is not to examine the reasons for, and extent of, variations in calorimeter response; instead the aim is to provide a global calibration on as small amount of data as possible to correct for potentially short range time variations.

As an automatic rolling calibration within OPR (see section 2.10.2), the procedure must be simple and robust, yet accurate.

\subsubsection{Parametrization of Photon Energy Correction}

The corrected measured photon energy, $\epsilon$, corresponding to a cluster with raw measured energy $E$ is given by

$$
\epsilon=E f(E, \theta),
$$


where $\theta$ is its measured polar angle. A parametrization of the energy correction function $f(E, \theta)$ is given by [32]

$$
f(E, \theta)=\exp \left(A_{0}+\sum_{i=1}^{N} A_{i} \ln ^{i} E+\sum_{i=1}^{N} B_{i} \cos ^{i} \theta\right),
$$

which takes into account shower leakage effects which depend on photon energy and $\theta$, since the crystal lengths differ over the polar angle range. The calibration described here uses only the energy dependent part of the correction, up to $i=2$. The simplified correction is therefore

$$
f(E)=\exp \left(A_{0}+A_{1} \ln E+A_{2} \ln ^{2} E\right),
$$

and requires the determination of three calibration coefficients $A_{0}, A_{1}, A_{2}$.

\subsubsection{Calibration Determination from $\pi^{0}$ Mass Constraint}

The coefficients in photon energy correction (3.4) are determined using the constraint imposed by the resulting calibrated $\pi^{0}$ invariant mass, where the $\pi^{0}$ decays to two photons with raw energies $E_{1}$ and $E_{2}$. The calibrated $\pi^{0} \rightarrow \gamma \gamma$ mass should peak at the nominal value, $m\left(\pi^{0}\right)=134.98 \mathrm{MeV} / c^{2}$. The calibrated $\pi^{0} \rightarrow \gamma \gamma$ mass is given by

$$
\begin{aligned}
m^{\prime}(\gamma \gamma)= & \sqrt{2 \epsilon_{1} \epsilon_{2}(1-\cos \alpha)} \\
= & m(\gamma \gamma) \times \\
& \exp \left(A_{0}+\frac{1}{2} A_{1}\left(\ln E_{1}+\ln E_{2}\right)+\frac{1}{2} A_{2}\left(\ln ^{2} E_{1}+\ln ^{2} E_{2}\right)\right)
\end{aligned}
$$

where $\alpha$ is the angle between the two photons and $m(\gamma \gamma)=\sqrt{2 E_{1} E_{2}(1-\cos \alpha)}$. It is convenient to use the variable $\ln m^{\prime}(\gamma \gamma)$ in which case the correction can be expressed

$$
\ln m^{\prime}(\gamma \gamma)=\ln m(\gamma \gamma)+\left(A_{0}+\frac{1}{2} A_{1}\left(\ln E_{1}+\ln E_{2}\right)+\frac{1}{2} A_{2}\left(\ln ^{2} E_{1}+\ln ^{2} E_{2}\right)\right) .
$$


The value of the coefficients are determined to be those which when applied, yield a peak in $\ln m^{\prime}(\gamma \gamma)$ at $\ln m\left(\pi^{0}\right)$. Equivalently, the distribution $\ln m^{\prime}(\gamma \gamma)-\ln m\left(\pi^{0}\right)$ should peak at zero. This constraint can be expressed by

$$
\ln \frac{m_{\text {peak }}(\gamma \gamma)}{m\left(\pi^{0}\right)}=-A_{0}-\frac{1}{2} A_{1}\left(\ln E_{1}+\ln E_{2}\right)-\frac{1}{2} A_{2}\left(\ln ^{2} E_{1}+\ln ^{2} E_{2}\right),
$$

where $m_{\text {peak }}$ is the position of the $\gamma \gamma$ mass peak. It is noticeable that to first order, the first coefficient $A_{0}$ can be estimated simply by the displacement of the raw peak position, $\ln \frac{m_{\text {peak }}(\gamma \gamma)}{m\left(\pi^{0}\right)}$, from zero. By quantifying the dependence of the $\pi^{0}$ peak position on photon energy, coefficients $A_{1}$ and $A_{2}$ can be estimated; this will be described in more detail later.

It is expected that the coefficients are correlated and cannot be calculated independently in one step. The approach taken is an iterative one, in which $A_{0}$ is estimated first and applied to the photon energies before $A_{1}$ is calculated. The coefficient $A_{2}$ that describes the higher order energy dependence is estimated after $A_{0}$ and $A_{1}$ have reached stable values in the iteration process. With sufficient iterations, all three coefficients should converge to stable values.

In all cases, the coefficient determination depends on the ability to calculate the position of the $\pi^{0}$ peak. The procedure for doing so is described in the next section.

\section{3 $\quad \pi^{0}$ Peak Position Determination}

\subsubsection{Overview}

The $\pi^{0}$ peak position calculation is central to the calibration method. To run automatically during OPR, a robust and reliable method of calculating the peak position (in the presence of sometimes high background) must be in place. 
Due to shower leakage, the $\pi^{0}$ mass peak is asymmetric. While statistical sampling effects and calibrations uncertainties have Gaussian effects on the photon energy resolution, the energy loss involved in shower leakage causes a tail on the lower side of the $\pi^{0}$ mass peak. In the the case of a symmetric $\pi^{0}$ peak and no background, the peak position could be calculated simply by the mean of the $\pi^{0}$ mass distribution. In the more realistic scenario, the asymmetric $\pi^{0}$ peak sits on a large background distribution as shown in Figure 3.1, but a combined fit to signal and background to determine the peak position cannot be practically be implemented in OPR.

The approach used here is to calculate a background-subtracted mean in a predefined signal range in the $\pi^{0}$ mass distribution, described in Section 3.3.3. To obtain the position of the peak or maximum, a correction was found from the $\pi^{0}$ mass spectrum in data, described in Section 3.3.4.

\subsection{2 $\pi^{0}$ Selection and Histogram Formation}

In the reconstruction of $\pi^{0}$ candidates, selections are kept minimal, so as to include the largest, most general sample of photons in the calibration. In this way, the correction obtained should be applicable to all photons across the energy scale. Events passing the multihadronic criteria are used, which include the requirement that at least three tracks are detected (in the ChargedTracks list, see Section 2.10.2) and that the ratio of the second to the zeroth Fox-Wolfram moments (see Appendix D) be less than 0.98. The photons are taken from the CalorNeutral list (see Section 2.10.2), and photons whose detection signals were affected by large amounts of electronic noise or data-flow damage are rejected.

Pairs of photons are then combined to form $\pi^{0}$ candidates, and histograms are made

of the invariant $\pi^{0}$ mass, in the variable $M=\ln \frac{m(\gamma \gamma)}{m\left(\pi^{0}\right)}$, after a suitable scaling to fix the range over which the peak position calculation is performed. 40 bins are used 
in each histogram, with the scaling factor chosen to define the range as $-0.5<M<$ $0.5\left(82 \mathrm{MeV} / c^{2}<m(\gamma \gamma)<223 \mathrm{MeV} / c^{2}\right)$. Figure 3.2 shows the histogram obtained from photon pairs where no cluster calibration has been applied. The lower and higher energy photon from each pair are required to exceed $30 \mathrm{MeV}$ and $255 \mathrm{MeV}$ respectively. The histogram range is divided into symmetrical (about $M=0$ ) signal and sideband regions for peak calculation purposes, as will be described later.

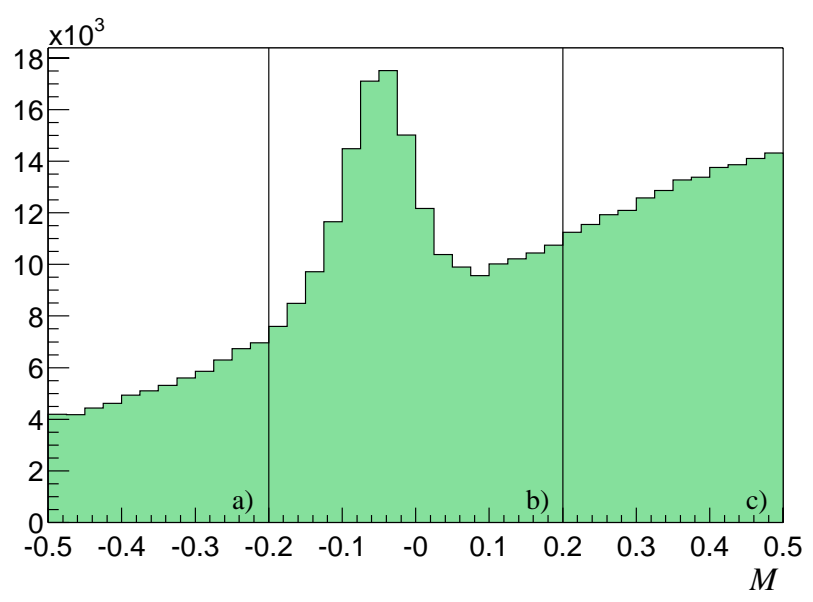

Figure 3.2: Reconstructed raw $\pi^{0} \rightarrow \gamma \gamma$ mass distribution in $M=\ln \frac{m(\gamma \gamma)}{m_{\pi^{0}}}$. For peak position calculation the distribution is divided into a) lower sideband b) signal band c) upper sideband. The lower (higher) energy photon is required to exceed $30 \mathrm{MeV}(255 \mathrm{MeV})$.

The separate energy threshold requirements on the two photons allows the retention of low energy photons while reducing the amount of background in the $\pi^{0}$ mass histogram. Figure 3.3 shows the $\pi^{0}$ mass distribution as the energy requirement is increased on the higher energy photon. Since the photon reconstruction energy threshold is $30 \mathrm{MeV}$, a separate threshold of at least $152 \mathrm{MeV}$ is required on the higher energy photon to ensure a physically meaningful $\pi^{0}$ energy.

A lower limit of $255 \mathrm{MeV}$ was chosen for the higher energy photon for these studies. This greatly reduces the combinatorial background to an almost linear shape (see 
Figure 3.2) in the range of $M$ considered. The lower limit on the other photon energy is $30 \mathrm{MeV}$ (the cluster reconstruction threshold).

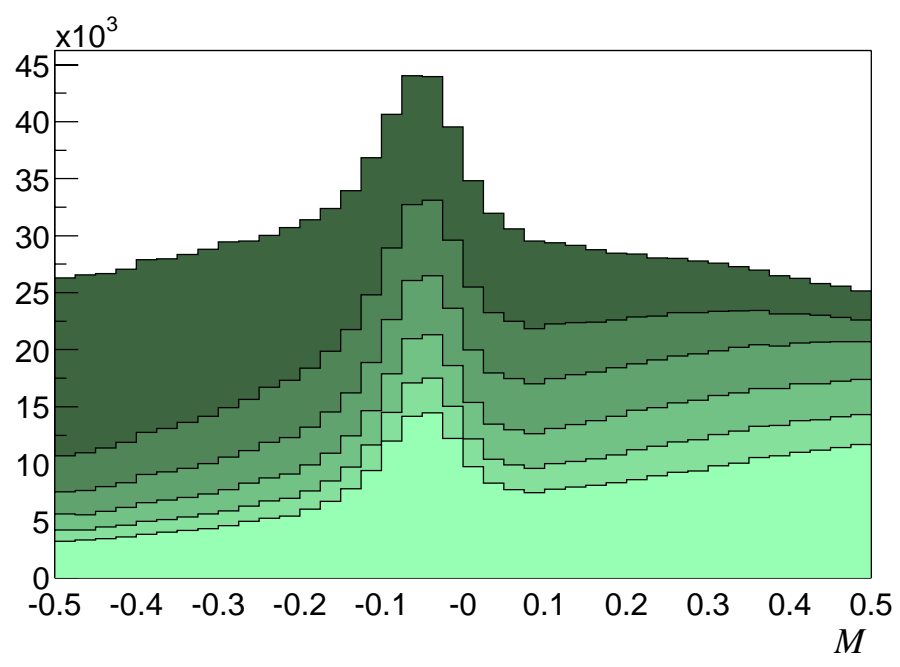

Figure 3.3: $\pi^{0} \rightarrow \gamma \gamma$ mass distributions with increasing (lighter colouring) energy photon thresholds. The higher energy photon is required to exceed $30 \mathrm{MeV}, 105 \mathrm{MeV}, 155 \mathrm{MeV}, 205 \mathrm{MeV}, 255 \mathrm{MeV}, 305 \mathrm{MeV}$.

\subsubsection{Background-Subtracted Mean Calculation}

A crude (under)estimate of the $\pi^{0}$ peak position is the mean of the $M$ distribution after background subtraction. A correction is applied later to better determine the peak position. The sidebands in $M$ are used to determine the background shape and thus extrapolate the background in the signal band.

Although with the selections described above, the background in $M$ appears approximately linear, this may not be so when $M$ is restricted to various $\pi^{0}$ energy intervals, as is required by the calibration procedure. The background shape may also change over the lifetime and evolutions of the BABAR data processing, for example if photon reconstruction algorithms are altered. For these reasons, a linear 
description of the background may not always be sufficient; instead a third order polynomial is used to account for variations in background shape.

In each histogram in $M$, the central 16 bins are defined as the signal band, the outer 24 bins are defined as the sidebands (see Figure 3.2). Although the peak shifts with respect to $M=0$ at each iteration of the calibration, the signal and sidebands definition remains the same throughout.

The background polynomial is fitted to the entries in the sideband bins, using the least squares method (see for example [33]). To facilitate the statistical error handling, the basis of polynomials $P_{n}(M), n=0,1,2,3$ used are chosen to be orthogonal over the data points $\left(M_{j}, N_{j}\right)$, where $M_{j}$ and $N_{j}$ are the mean $M$ value and number of entries respectively in sideband bin $j$. The orthogonality condition is

$$
\sum_{j}^{S B} \frac{P_{n}\left(M_{j}\right) P_{m}\left(M_{j}\right)}{\sigma_{j}^{2}}=0 \quad \text { unless } n=m,
$$

where $\sum_{j}^{S B}$ denotes a sum over sideband bins, and $\sigma_{j}=\sqrt{N_{j}}$ is the error on the number of bin entries. The background polynomial that is then fit can be expressed

$$
f_{b g}(M)=\sum_{n=0}^{3} a_{n} P_{n}(M),
$$

and has the advantage that the coefficients $a_{n}$ obtained from the fit are uncorrelated. Details of the extraction of the coefficients $a_{n}$ and their errors are given in Appendix B.

The mean is calculated in the signal band after background subtraction, using $f_{b g}(M)$. The mean without background subtraction in the signal bins is given by

$$
\mu_{R}=\frac{\sum_{i}{ }^{S} M_{i} N_{i}}{\sum_{i}{ }^{S} N_{i}}
$$

where $\sum_{i}^{S}$ denotes a sum over signal bins. (3.10) is just the unbinned average of 
the entries in the signal band. After background subtraction, the mean is given by

$$
\mu=\frac{\sum_{i}^{S} M_{i} N_{i}-\sum_{i}^{S} M_{i} f_{b g}\left(M_{i}\right)}{\sum_{i}^{S} N_{i}-\sum_{i}^{S} f_{b g}\left(M_{i}\right)},
$$

$\mu$ is therefore a function of the raw mean $\mu_{R}$ and the coefficients of $f_{b g}\left(M_{i}\right), a_{n}$. When calculating the error on this quantity, the propagation of the errors is greatly simplified by the orthogonal coefficients, leading to

$$
\sigma_{\mu}=\left(\frac{\partial \mu}{\partial \mu_{R}}\right) \sigma_{\mu_{R}} \oplus\left(\frac{\partial \mu}{\partial a_{0}}\right) \sigma_{a_{0}} \oplus\left(\frac{\partial \mu}{\partial a_{1}}\right) \sigma_{a_{1}} \oplus\left(\frac{\partial \mu}{\partial a_{2}}\right) \sigma_{a_{2}} \oplus\left(\frac{\partial \mu}{\partial a_{3}}\right) \sigma_{a_{3}},
$$

with no covariance terms; only the derivatives $\frac{\partial \mu}{\partial a_{n}}$ must be obtained.

A typical background fit is shown in Figure 3.4, where all photon pairs satisfying the energy thresholds are included. In this example 69,134 multihadronic events were used, giving a background-subtracted mean value of $\mu=-0.066 \pm 0.001$. This corresponds to a mean value of $126.38 \pm 0.13 \mathrm{MeV} / c^{2}$, in $m(\gamma \gamma)$.

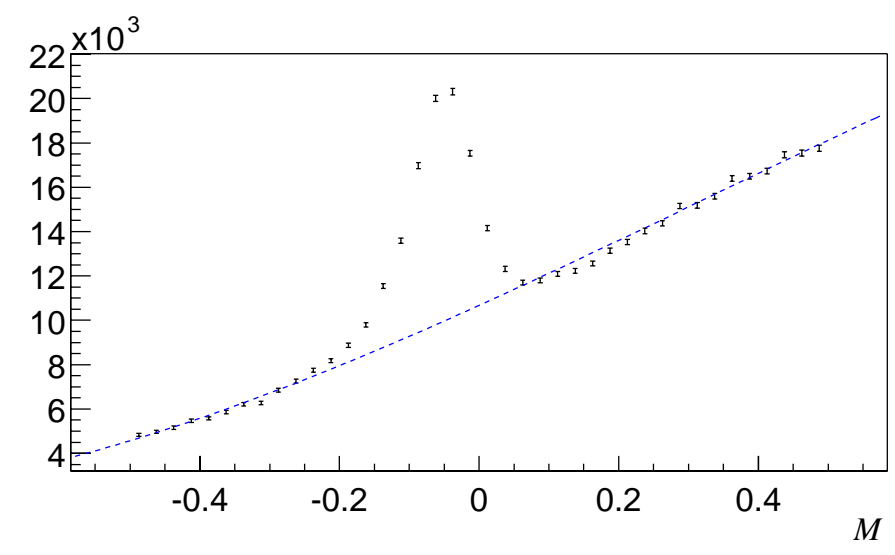

Figure 3.4: Background fit (using the method described in the text) to the $\pi^{0}$ mass distribution. Here, raw photon energies are used, imposing only the energy thresholds described in the text. The background-subtracted mean is found to be $\mu=-0.066 \pm 0.001$.

The mean calculation provides a reliable estimate that can be used in an automatic 
algorithm. Next, a correction is required to relate the position of the mean to that of the peak.

\subsubsection{Peak Calculation from Mean}

The peak or maximum of $\pi^{0}$ mass distribution has a larger $M$ value than the mean due to its asymmetry. Thus, a calibration that corrects the mean $\pi^{0}$ mass to $134.98 \mathrm{MeV} / \mathrm{c}^{2}$ (or $\mu=0$ ) will result in a peak position higher than the desired value.

The shape of the $\pi^{0}$ mass peak may be described by the 'Novosibirsk' [34] function, a parametrization of the logarithmic normal distribution given by

$$
f_{\text {Nov }}(m)=A \exp \left(\frac{-\ln ^{2}\left(1+K\left(m-m_{0}\right)\right)}{2 t^{2}}-\frac{t^{2}}{2}\right),
$$

where $K=\frac{\sinh (t \sqrt{\ln 4})}{\sqrt{\ln 4} \sigma}$. A is the normalisation, $m_{0}$ is the position of the maximum, $\sigma$ is the width $(\mathrm{FWHM} / 2 \sqrt{\ln 4})$, and $t$ is the tail parameter. The mean of the Novosibirsk function is given by

$$
m_{0}+\frac{\sigma \sqrt{\ln 4}}{\sinh (t \sqrt{\ln 4})}\left(e^{3 t^{2} / 2}-1\right) .
$$

Using the Novosibirsk function rather than the conventional logarithmic normal function, one can directly extract the $\pi^{0}$ peak position and width from a fit to the $m(\gamma \gamma)$ distribution (with a suitable additional function to describe the background). However, it is impractical to implement a peak finding routine based on these kind of fits on a run-by-run basis in OPR.

Instead, the simple mean calculation described above is combined with a mean-topeak correction using the Novosibirsk function. A relationship was sought between the Novosibirsk peak and the mean that should hold generally for data. Unfortunately a relationship of this sort cannot be expected to hold for all $\pi^{0}$ energies, since 
the tail on the $\pi^{0}$ mass peak varies with $\pi^{0}$ energy. A relationship was sought of the form

$$
m_{\text {peak }}(\gamma \gamma)-m_{\text {mean }}(\gamma \gamma)=f\left(E_{\pi^{0}}\right) .
$$

If such a relationship existed, then to obtain the correct peak $\pi^{0}$ mass the target of the calibration should be to achieve $m_{\text {mean }}(\gamma \gamma)=134.98-f\left(E_{\pi^{0}}\right)$ which may be substituted for $m\left(\pi^{0}\right)$ in (3.7).

To obtain such a relationship, a fit of the sum of a Novosibirsk and a second order polynomial was performed to the $m(\gamma \gamma)$ distribution in several bins of $\pi^{0}$ energy. For each energy bin, the peak-mean separation was measured using (3.14). A linear fit to data from April 2001 (runs 19346-19420, $10^{6}$ events) [35] was performed, with the result

$$
f\left(E_{\pi^{0}}\right)=0.002-0.0005\left(E_{1}+E_{2}\right) \mathrm{GeV} .
$$

This was verified for data taken at a separate period in the BABAR data sample (February 2000, $\sim 7 \times 10^{5}$ events). Figure 3.5 shows the fits to the $\pi^{0}$ mass in individual energy bins. A linear fit to the $m_{\text {peak }}(\gamma \gamma)-m_{\text {mean }}(\gamma \gamma)$ points (see Figure 3.6) gave

$$
f\left(E_{\pi^{0}}\right)=0.00177( \pm 0.00007)-0.00053( \pm 0.00005)\left(E_{1}+E_{2}\right) \mathrm{GeV},
$$

which is consistent with the previous result (3.16).

\subsection{Calibration Coefficient Extraction}

\subsubsection{Energy Binning}

As will be explained, extraction the coefficients $A_{1}$ and $A_{2}$ involves calculating the $\pi^{0}$ peak position in various bins of $\ln E_{1}+\ln E_{2}$. Choosing a range of $\ln E_{1}+\ln E_{2}$ which 

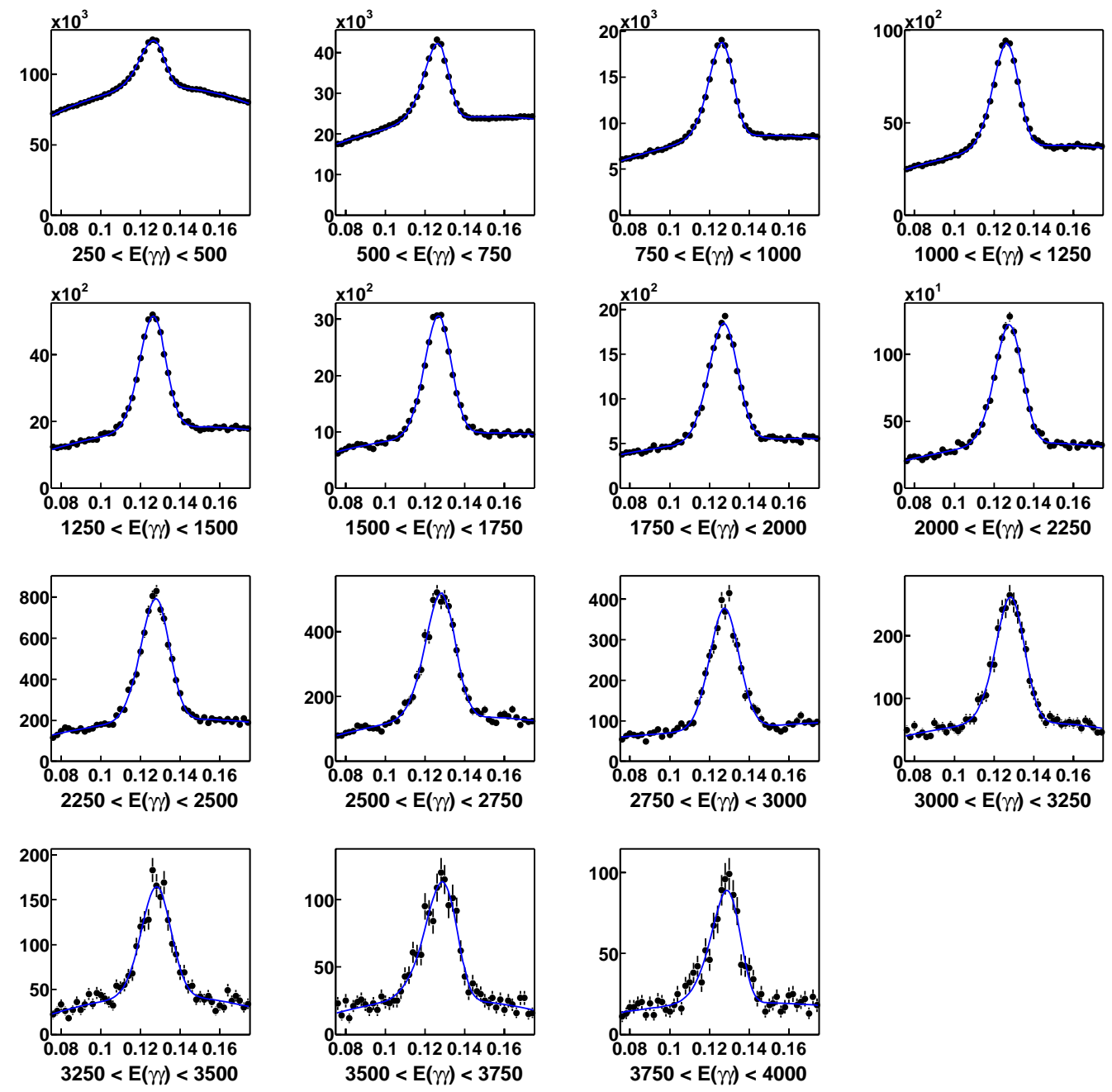

Figure 3.5: Raw $\pi^{0}$ mass distribution (horizontal axes units are $\mathrm{GeV} / \mathrm{c}^{2}$ ) in increasing bins of $\pi^{0}$ energy (units of $E(\gamma \gamma)$ are $M e V$ ). A fit of the sum of a Novosibirsk function (see text) and a second order polynomial is superimposed.

covers the full range of $\pi^{0}$ energies is important. The distribution of $\ln E_{1}+\ln E_{2}$ is very correlated with the $\pi^{0}$ energy; the correlation and boundaries imposed by the lower and higher energy photon thresholds is shown in Figure 3.7.

The bins boundaries in $\ln E_{1}+\ln E_{2}$ were chosen to be -5.0, -3.0, -2.4, -2.2, -2.0, $-1.5,-1.0,-0.5,0.0,0.5,1.0$, defining a total of 10 bins. The optimal choice of bin boundaries involves several factors. Choosing enough bins to parametrize $\pi^{0}$ mass 


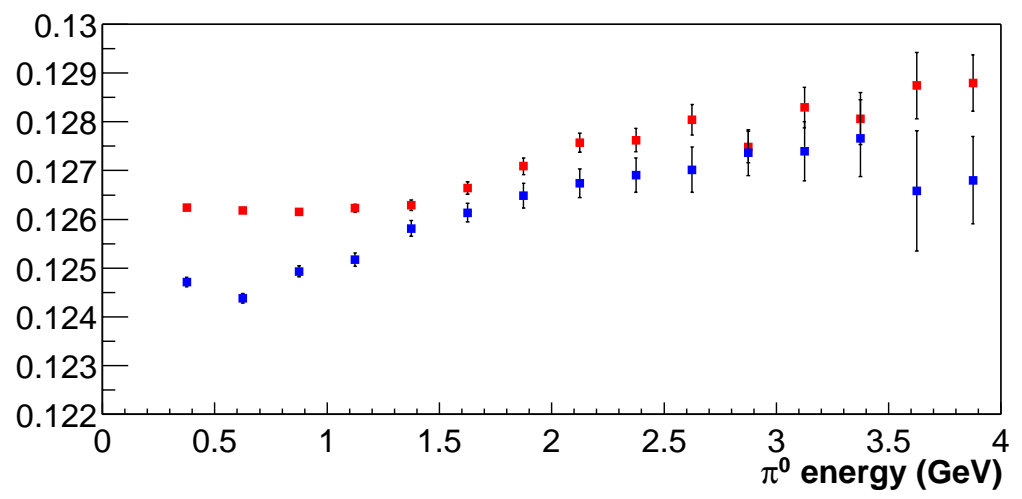

(a)

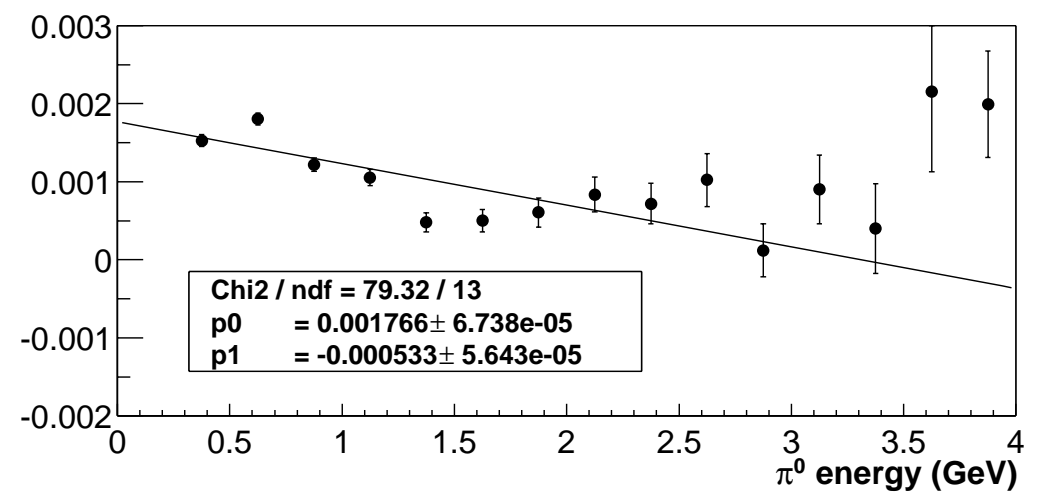

(b)

Figure 3.6: (Top) The peak (red points) and mean (blue points) extracted from the Novosibirsk fits to the $\pi^{0}$ mass distributions (Figure 3.5). (Bottom) the peak-mean difference; a linear fit with parameters $p 0$ (constant) and $p 1$ (slope) is superimposed. The units of the vertical scales are $\mathrm{GeV} / \mathrm{c}^{2}$.

behaviour while having sufficient statistics in each bin is one consideration. The spacing of the bin boundaries would ideally be set such that each bin contained the same number of entries. However at low values of $\ln E_{1}+\ln E_{2}$, the background is high and a reliable peak position can only be determined allowing the lowest $\ln E_{1}+\ln E_{2}$ bins to contain a larger fraction of the total energy range. A study to find the optimal bin boundaries has not yet been performed. 


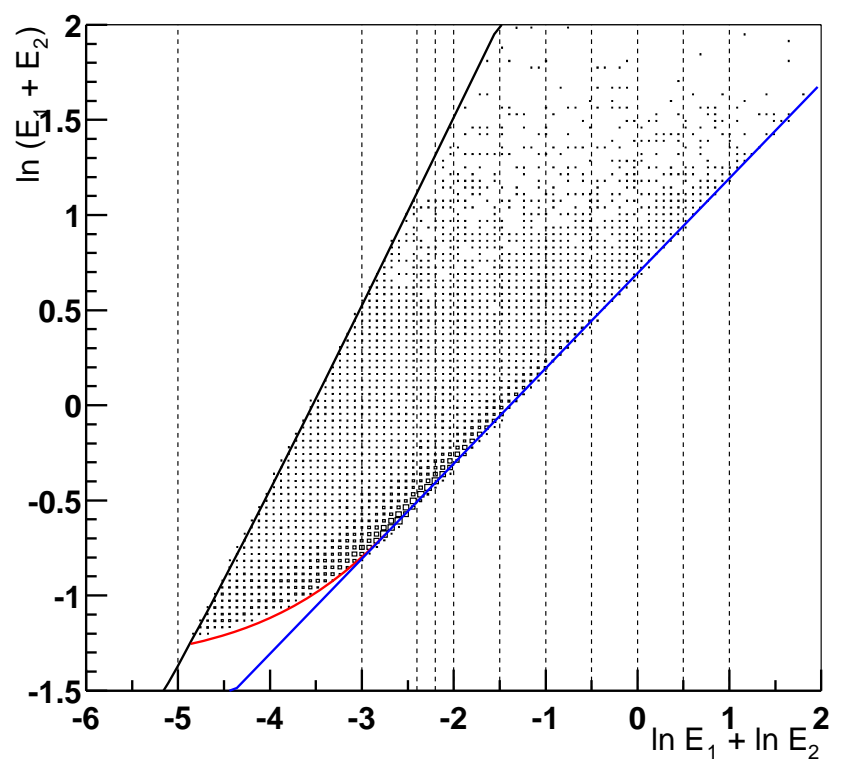

Figure 3.7: Correlation between $\pi^{0}$ energy $\left(\ln \left(E_{1}+E_{2}\right)\right)$ and $\ln E_{1}+\ln E_{2}$. The red, blue and black lines indicate the boundaries imposed by the photon energy selections. The dashed lines indicate the bin boundaries used in the calibration covering a total range of $-5.0<\ln E_{1}+\ln E_{2}<1.0$.

\subsubsection{Calculation of Calibration Coefficients}

As mentioned in Section 3.2.2, the calibration coefficients are calculated iteratively, so as to account for correlations between the coefficients. This section describes how each coefficient is calculated in a given iteration. The examples shown use data from a typical run taken in February 2002, which has 69,134 multihadronic events.

The first coefficient is simply extracted by the shift of the raw peak in $M, A_{0}=$ $-M_{\text {peak }}$, as mentioned in Section 3.2.2. An illustration of the $M_{\text {peak }}$ calculation is given by Figure 3.4.

The coefficient $A_{1}$ can be determined from a linear fit to the $M_{\text {peak }}$ vs. $\ln E_{1}+\ln E_{2}$ 
distribution. This makes the assumptions that the $\ln ^{2} E_{1}+\ln ^{2} E_{2}$ correction is small in comparison. $M_{\text {peak }}$ is calculated in several bins of $\ln E_{1}+\ln E_{2}$ defined in Section 3.4.1. This is demonstrated by Figure 3.8. A significant slope is seen in the data, and its value, multiplied by -2 (see (3.7)), gives $A_{1}$. The slope is computed by the least squares method in a similar way to the 3rd order background fit to the $\pi^{0}$ mass spectrum (however, orthogonal polynomials are not used). For the example shown in Figure 3.8, it should be noted that $M_{\text {peak }}$ in the lowest energy bin departs significantly from the overall linear behaviour of $M_{\text {peak }}$ vs. $\ln E_{1}+\ln E_{2}$. This is due to the poor description of the background in the $M$ signal region, caused by a large offset of the peak from $M=0$. This systematic effect is largest in the first iteration, when no $A_{0}$ has been applied; it does not persist to the same extent throughout all calibration iterations.

The coefficient $A_{2}$ can be extracted from a quadratic fit to the $M_{\text {peak }}$ vs. $\ln E_{1}+\ln E_{2}$ distribution, where the two photon energies are approximately equal (the criteria that is used is $\left.\left|\ln E_{1}-\ln E_{2}\right|<0.2\right)$. From this condition, it follows that

$$
\ln ^{2} E_{1}+\ln ^{2} E_{2}=\frac{1}{2}\left(\ln E_{1}+\ln E_{2}\right)^{2},
$$

and so (3.7) becomes

$$
\ln \frac{m_{\text {peak }}(\gamma \gamma)}{m_{\pi^{0}}}=-A_{0}-\frac{1}{2} A_{1} x-\frac{1}{4} A_{2} x^{2},
$$

where $x=\ln E_{1}+\ln E_{2}$. Thus the quadratic coefficient of the fit, multiplied by -4, gives $A_{2}$. This is demonstrated in Figure 3.9. Due to the $\left|\ln E_{1}-\ln E_{2}\right|<0.2$ condition, the sample size is reduced, and the statistical power of the $A_{2}$ calculation is lower than for $A_{1}$. A significant quadratic dependence of $M_{\text {peak }}$ on $\ln E_{1}+\ln E_{2}$ is not found for this example.

In any given iteration, the lowest order coefficient that deviates significantly ( $>3 \sigma$, where $\sigma$ is the statistical error on the deviation) from its previous value is adjusted to its newly calculated value. Hence a maximum of one coefficient is updated on 

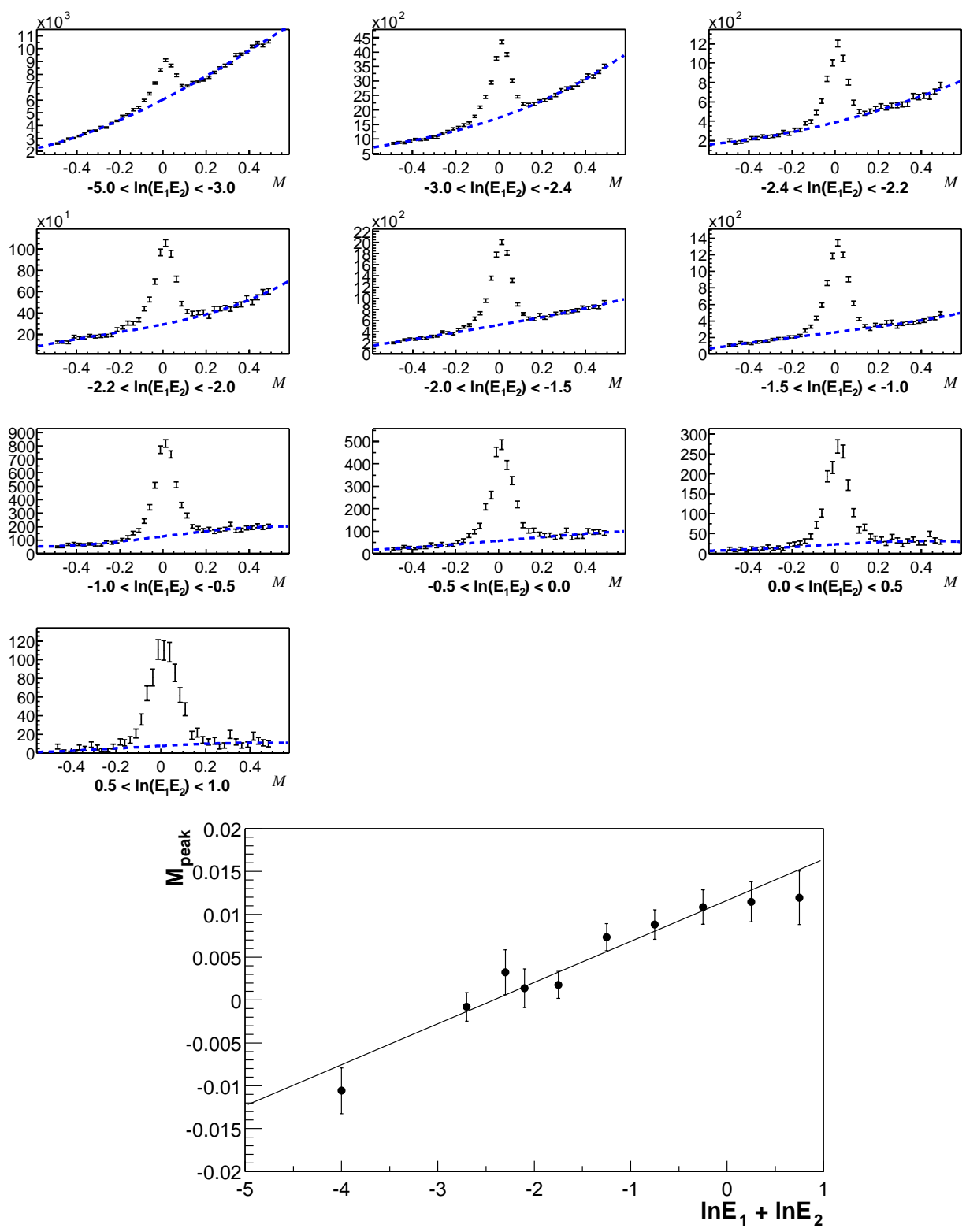

Figure 3.8: The $M$ distribution (top) in bins of $\ln E_{1}+\ln E_{2}$ with background fits superimposed, after applying a stable $A_{0}$ calibration coefficient. The calculated peak positions $M_{\text {peak }}$ are plotted (bottom) against the centre of each $\ln E_{1}+\ln E_{2}$ bin; $A_{1}$ is extracted from a linear fit to these points.

each iteration. Thus $A_{0}$ is applied first, and in the following iterations a mutual stability of $A_{0}$ and $A_{1}$ is required before $A_{2}$ is applied. 

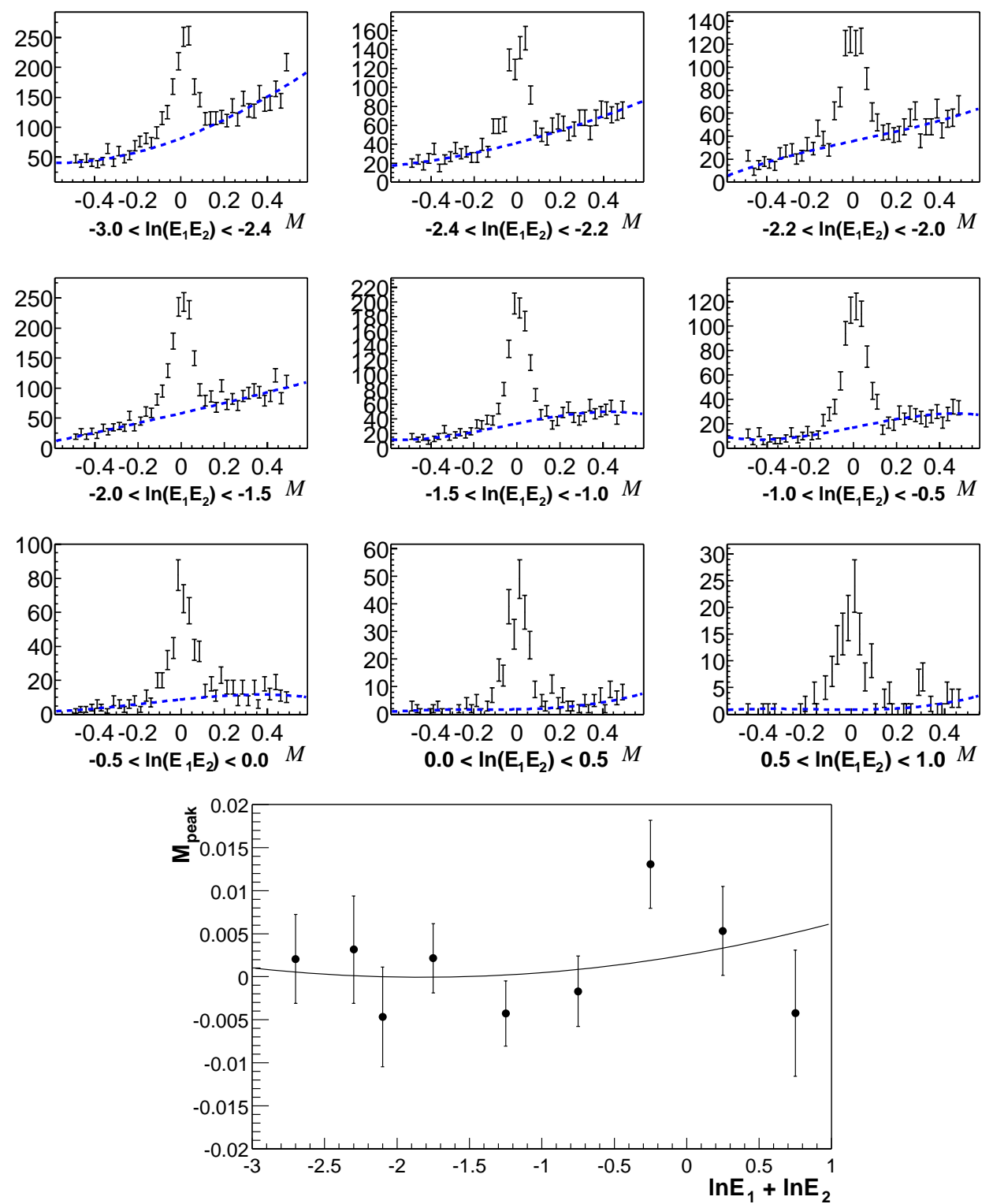

Figure 3.9: The $M$ distribution (top) in bins of $\ln E_{1}+\ln E_{2}$ for $E_{1} \approx E_{2}$ with background fits superimposed, after applying stable $A_{0}$ and $A_{1}$ calibration coefficients. The calculated peak positions $M_{\text {peak }}$ are plotted (bottom) against the centre of each $\ln E_{1}+\ln E_{2}$ bin; $A_{2}$ is extracted from a quadratic fit to these points.

Based on the examination of the calibration performance in individual runs, it was determined that at least 50,000 multihadronic events were required to provide a 
energy dependent cluster calibration.

\subsection{Calibration Performance}

The performance of the calibration method can be demonstrated for a given run in the $B A B A R$ data sample by applying the algorithm as described above and observing the calibrated $\pi^{0}$ mass distributions across the $\pi^{0}$ energy range. The corrected $\pi^{0}$ peak position should be equal to the nominal $\pi^{0}$ mass.

As an example, the calibration applied to run 26000 (February 2002, containing 69,134 multihadronic events). The values of the coefficients at each iteration are shown in Table 3.1. Four iterations were required until stable coefficients $A_{0}$ and $A_{1}$ were obtained. When this calibration was applied and the coefficients recalculated, they were found to be $A_{0}^{\prime}=0.000348 \pm 0.000851, A_{1}^{\prime}=0.000189 \pm 0.000569, A_{2}^{\prime}=$ $-0.00292 \pm 0.00714$, all consistent with zero.

Table 3.1: Coefficients applied at each iteration in the calibration of run 26000 (see text).

\begin{tabular}{c|c|c|c}
\hline Iteration & $A_{0}$ & $A_{1}$ & $A_{2}$ \\
\hline \hline 0 & 0 & 0 & 0 \\
1 & 0.0664 & 0 & 0 \\
2 & 0.0626 & 0 & 0 \\
3 & 0.0626 & -0.00961 & 0 \\
4 & 0.0514 & -0.00961 & 0 \\
\hline
\end{tabular}

The fitted $\pi^{0}$ mass in the energy intervals (defined in Section 3.3.4) is shown before and after the calibration in Figure 3.10. In most energy bins the corrected $\pi^{0}$ peak 
position is within $0.5 \mathrm{MeV}$ of the nominal $\pi^{0}$ mass. The $\pi^{0}$ resolution (defined as the Novosibirsk width divided by the peak position) is also shown in Figure 3.10 and does not visibly improve after calibration.
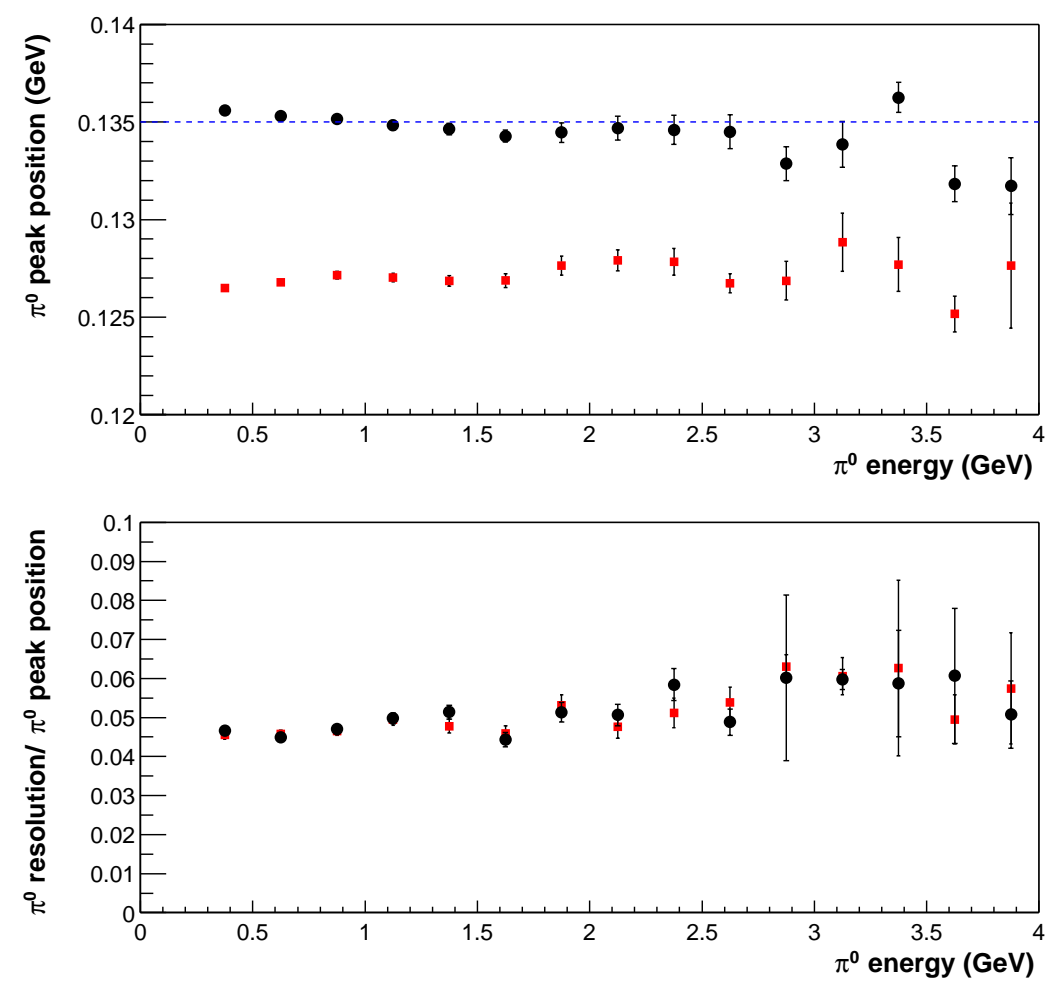

Figure 3.10: Fitted $\pi^{0}$ peak position (top) and resolution (normalised to the peak position) (bottom) before (red points) and after (black points) calibration.

The use of a correction factor to $A_{0}$ when applying $A_{1}$, to account for the correlation between the two coefficients was previously employed ${ }^{1}$. An adjustment of $A_{0} \rightarrow$ $A_{0}+1.2 A_{1}$ was made whenever a significant value of $A_{1}$ was computed and applied. If the calibration for run 26000 is performed again implementing this adjustment, the final coefficient values are $A_{0}=0.0511, A_{1}=-0.00961, A_{2}=0$, obtained in 3 iterations. Although this kind of correction may be useful in reducing the amount of iteration required, it has yet to be shown to be valid in all cases.

\footnotetext{
${ }^{1}$ Implemented by R. J. Barlow.
} 
The performance of the $\pi^{0}$ calibration over large periods of data taking, operating in OPR as a rolling calibration rather than as a 'standalone' routine as described above has yet to be investigated.

\subsection{Statistical and Systematic Errors}

Errors in the calibration affect both the calibrated photon energies and the resulting position of the $\pi^{0}$ mass peak. The following sections attempt to estimate the size of statistical and systematic errors.

\subsubsection{Statistical Errors}

The error on the calibrated photon energy (3.2), propagated from the statistical errors on calibration coefficients $A_{i}$ depends on the degree of correlation between the coefficients.

A positive correlation between $A_{0}$ and $A_{1}$ is observed from the example in Section 3.5 (after applying a negative value of $A_{1}, A_{0}$ is correspondingly decreased). Ignoring the $A_{2}$ coefficient, the fractional error on the calibrated energy for fully correlated coefficients is given by

$$
\frac{\sigma_{E}}{E}=\left|\sigma_{A_{0}}+\ln E \sigma_{A_{1}}\right| .
$$

Taking $\sigma_{A_{0}}=0.00085$ and $\sigma_{A_{1}}=0.00056$ from the calibration performed in Section 3.5 , it follows that the calibrated energy error is $0.11 \%$ at $E=30 \mathrm{MeV}$, and falls at higher energies.

The statistical error on $A_{2}$ computed in Section 3.5 is rather large. If this is included in the propagated error on the calibrated energy the total fractional error rises to 
$\sim 8 \%$ at $30 \mathrm{MeV}$. However it is likely that $\sigma_{A_{2}}$ is poorly (over)estimated due to an insufficient sample size. The actual results from the calibration, (where the fitted $\pi^{0}$ mass is within $0.5 \mathrm{MeV}$ of the desired value) suggests that the result $A_{2}=0$ is more reliable than the the error $\sigma_{A_{2}}$ suggests.

To determine the $A_{2}$ coefficient to an accuracy such that it does not seriously degrade the accuracy of the calibrated photon energy, a much larger data sample than 50,000 events would be required.

\subsubsection{Systematic Errors}

An obvious source of systematic error is involved in the peak-mean correction described in Section 3.3.4. The resulting contribution to the error on $A_{0}$ can be estimated by using the approximate constraint (from (3.7))

$$
\ln \frac{m_{\text {peak }}(\gamma \gamma)}{m\left(\pi^{0}\right)} \approx-A_{0},
$$

where $m\left(\pi^{0}\right)$ is replaced by $m\left(\pi^{0}\right)-C-D\left(E_{1}+E_{2}\right)$ to encode the peak-mean correction. From (3.21) the error on $A_{0}$ is given by

$$
\sigma_{A_{0}}=\frac{\sigma\left(m\left(\pi^{0}\right)\right)}{m\left(\pi^{0}\right)},
$$

and in terms of the peak-mean correction, $\sigma\left(m\left(\pi^{0}\right)\right)$ is given by

$$
\sigma\left(m\left(\pi^{0}\right)\right)=\sqrt{\sigma_{C}^{2}+\sigma_{D}^{2}\left(E_{1}+E_{2}\right)^{2}} .
$$

Estimating the errors on the parameters $C$ and $D$ by taking the difference between those obtained in (3.16) and (3.17), added in quadrature to their statistical errors yields $\sigma_{C}=0.00024$ and $\sigma_{C}=0.00006$. Thus the error on $A_{0}$ is dominated by the error on $C$ giving $\sigma_{A_{0}}(s y s t) \approx 0.002$, approximately twice as large as the statistical error alone. 
Systematic errors on the peak position evaluation due to poor description of the background can be large. Poor statistics in individual $\ln E_{1}+\ln E_{2}$ bins and the initial offset of the $\pi^{0}$ peak from $M=0$ both affect the background estimation. This is even apparent in the calculation of the $A_{0}$ coefficient (see Table 3.1), where two iterations are required to achieve a stable value of $A_{0}$. While the systematic effects due to the peak offsets is greatly reduced after just one iteration, the errors due to poor statistics (particularly in the calculation of the $A_{2}$ coefficient) can only be resolved by using a larger data sample for calibration.

\subsection{Rolling Calibration}

Providing a run-by-run $\pi^{0}$ calibration is the goal of the calibration method described here. The simple 'peak finding' routine described in this chapter enables the calibration to be performed quickly and robustly and thus should be suitable to act as a rolling calibration.

\subsubsection{Overview of Rolling Calibration}

The need to determine the coefficients in several iterative steps poses a particular problem in integrating the algorithm into the OPR calibration sequence. This sequence is performed once for each run and thus iterative calibration, starting from the raw cluster energies is not possible.

However, the iterative improvement of coefficient estimation is still possible in the context of rolling calibration, where the calibration operates on a data set which is continually updated with events from new runs while discarding events from old runs. This is achieved by the use of an OPR scalar, a device that allows the storage 
of the necessary objects for performing the calibration ( $M$ histograms) from an arbitrary number of processed events. As the runs of raw data are processed the scalar accumulates events, at the end of each run the number of events in the scalar size can be checked and if it exceeds the number required for a calibration, the coefficients may be recalculated and adjusted appropriately. Once the scalar is 'full' (contains enough events for a calibration) it is then updated at the end of each run. Any new coefficients are written to the BABAR calibration database where they can be applied later during physics analysis reconstruction. The time validity interval of the coefficients coincides with the start time of the period of data from which they were calculated and extended indefinitely into the future. This validity interval may then be partially overwritten by subsequent changes in the coefficients as the rolling calibration processes new data.

Technically speaking, the $M$ histograms are not automatically updated at the end of each run, but stored. When the size of the scalar is checked at the end of a run and found to be sufficiently large, the stored histograms are merged. This is known as the temporal merge.

If the uncalibrated $\pi^{0}$ peak position does not change suddenly from run to run for a given data sample, the effect of rolling calibration will be to iteratively calculate the coefficients for that data sample. Of course, if the rolling calibration commences with all the coefficients set to zero the first 3 or 4 coefficient updates will not provide an optimal calibration.

However there is subtle problem inherent in rolling calibration which was discovered during testing. In the situation described above, $M$ histograms from different calibrations may be stored and merged together, resulting in a meaningless $\pi^{0}$ peak position. The problem may be elucidated by the following example. Consider a rolling calibration process where the first run processed (starting at time $t_{0}$ ) has no calibration. As the processing continues $M$ histograms with no applied calibration 
are stored for each run in the scalar. When the scalar reaches the required size, the histograms are merged together and the calibration executed. The $A_{0}$ coefficient is written to the database with a validity beginning at $t_{0}$ and extending to $t=\infty$. The histograms already stored are unaffected, but the histograms collected from the next run will have the new calibration applied. If that run contains few events, the calibrated histograms (with few entries) will be merged with all the other previously collected uncalibrated histograms. Thus, the measured $\pi^{0}$ mass will not appear to have changed from its uncalibrated position, and $A_{0}$ will be adjusted by a correction approximately the same size as the previously calculated $A_{0}$. The new coefficient (approximately twice the size it should be) will be written to the database overlapping the validity interval of the old coefficient.

To circumvent the problem described above, temporal merging was prevented unless all the stored histograms corresponded to the same calibration ${ }^{2}$. This was achieved by storing the values of the coefficients for each run along with the $M$ histograms; the calibration of each stored histogram can be checked before permitting the temporal merge.

The minimum scalar size was set to 50,000 multihadronic events, the amount required for an energy dependent calibration as described in Section 3.4.2. An illustration of rolling calibration is shown in Table 3.2, where the information was gathered during a test of the calibration using runs taken during March 2001. The size of the scalar only increases when the set of coefficients is constant, as required.

\subsubsection{Pass 1 Calibration}

Before September 2002, calibrations operated alongside normal event reconstruction in OPR, and the same events were used for both functions.

\footnotetext{
${ }^{2}$ Implemented by J. H. Weatherall.
} 
Chapter 3. Calibration of the Electromagnetic Calorimeter with $\pi^{0} \rightarrow \gamma \gamma$ Events

Table 3.2: Coefficients calculation in rolling calibration. The values of the coeffcients, the number of multihadronic 'MH' events, and the scalar size are shown for a sequence of runs. The size of the scalar only increases when the coefficients are fixed.

\begin{tabular}{c|c|c|c|c|c}
\hline Run Number & $A_{0}$ & $A_{1}$ & $A_{2}$ & \#MH Events & Scalar Size \\
\hline \hline 18905 & 0.051676 & 0 & 0 & 78815 & 78815 \\
18906 & 0.051676 & 0 & 0 & 29880 & 108695 \\
18912 & 0.051676 & 0 & 0 & 4887 & 113582 \\
18913 & 0.051676 & 0 & 0 & 14872 & 128454 \\
18914 & 0.051676 & 0 & 0 & 1079 & 50718 \\
18915 & 0.051676 & 0 & 0 & 18938 & 69656 \\
18917 & 0.044264 & 0 & 0 & 44219 & 0 \\
18918 & 0.044264 & 0 & 0 & 24257 & 68476 \\
18919 & 0.052411 & 0 & 0 & 20029 & 0 \\
18921 & 0.052411 & 0 & 0 & 1700 & 0 \\
18922 & 0.052411 & 0 & 0 & 3656 & 0 \\
18923 & 0.052411 & 0 & 0 & 11477 & 0 \\
18925 & 0.052411 & 0 & 0 & 27373 & 64235 \\
18926 & 0.041087 & 0 & 0 & 81405 & 81405 \\
\hline
\end{tabular}

Typically early runs in the BABAR data sample vary quite significantly in size, with the largest corresponding to around 50,000 multihadronic events and so a scalar size sufficient for $\pi^{0}$ calibration would need to be collected from multiple runs. More recent runs (collected after January 2002) contain typically more than 50,000 multihadronic events and so a calibration as described above could be calculated for each run.

Recently however, the organisation of calibrations and reconstruction changed to allow calibration to be applied before final event reconstruction. This is called 
the Pass 1 and Pass 2 prompt reconstruction. In Pass 1 a small subset of events is collected from each run and used to calculate the calibration coefficients for the various online calibrations, which can then be applied to the basic detector quantities before higher level reconstruction is performed in Pass 2, which processes all events for each run.

In the Pass 1 scheme, up to 7,000 multihadronic events are collected for recent runs (after January 2002, before July 2002). Thus a calibration based on 50,000 events requires data collected from at least 7 runs. This number will decrease with increased luminosity of the PEP-II collider.

\subsubsection{Stability Issues}

The stability of $\pi^{0}$ calibration in rolling calibration has still to be established.

Changes in the measured raw $\pi^{0}$ mass, subject only to single crystal calibration, may lead to instability of the coefficients and an inaccurate calibration over the period of instability. The behaviour of the measured $\pi^{0}$ mass has not been studied in detail over the whole $B A B A R$ dataset. Some (limited) information can be obtained from the studies in this chapter. For example, the plots of $\pi^{0}$ mass as a function of $\pi^{0}$ energy in data taken in February 2000 (Figure 3.6) and February 2002 (Figure 3.10) show a similar offset of the raw $\pi^{0}$ mass, although the energy dependence is less pronounced in the 2002 sample.

Changes in the $\pi^{0}$ mass behaviour may result from effects in other calibrations (electronic and single crystal) whose algorithms may evolve while the data is collected and processed. Other changes in the BABAR software and hardware, such as changes in photon-cluster reconstruction, introduction of digital filtering to the crystal readouts, replacement of some components of the front-end-electronics, may 
all impact the $\pi^{0}$ mass peak ${ }^{3}$. These effects may result in sudden changes in the $\pi^{0}$ mass distribution. Longer term changes may result from radiation damage to the crystals.

The complicated interplay of all the calibrations with potential $\pi^{0}$ mass fluctuations is poorly understood at present. While in theory the calibration should be able to operate in gradually changing conditions, sudden fluctuations may lead to dangerous instability in the rolling calibration. This has indeed been observed in the early tests of the rolling $\pi^{0}$ calibration. In such an event, the only current remedy is to resort to a manual 'off-line' application of the calibration to the affected period of data.

It is hoped that in the future the raw $\pi^{0}$ mass behaviour and its stability will become better understood, and that the rolling $\pi^{0}$ calibration is modified to account for unpredictable behaviour.

\subsection{Summary}

A reasonably robust and accurate $\pi^{0}$ peak finding algorithm has been developed, that has been demonstrated to calculate the $\pi^{0}$ mass to an accuracy of $\sim 0.5 \mathrm{MeV}$ and provide a corresponding calibration to photon energies from $2 \mathrm{GeV}$ down to the lowest $\pi^{0}$ energies. The performance results are however preliminary, based on a standalone iterative application of the calibration algorithm to a fixed set of data.

The performance of the $\pi^{0}$ as a rolling calibration has still to be established. Currently the global calibration of photon energies in the $E<0.8 \mathrm{GeV}$ region is performed using a manual $\pi^{0} \rightarrow \gamma \gamma$ calibration, not performed in OPR. This calibration uses the same form of parametrization of the photon energy correction, but a

\footnotetext{
${ }^{3}$ For details of these kinds of modifications, see [26].
} 
different method of extracting the coefficients, and a larger number of coefficients (including those that encode a polar angle dependent correction) [36]. This calibration is performed for large periods of data in which the $\pi^{0}$ mass behaviour is assumed to be stable, rather than on a run-by-run basis. It is this calibration that is applied to the photon energies in the $B \rightarrow \eta_{c} K, \eta_{c} \rightarrow K^{+} K^{-} \pi^{0}$ analysis described in Chapters 4 and 5. 


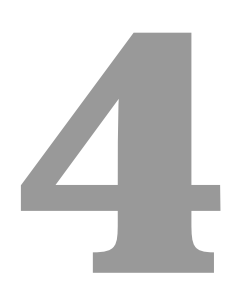

\section{Selection of $B \rightarrow \eta_{c} K$ Candidates}

\subsection{Introduction}

The analysis described in Chapter 5 measures the branching fractions of $B^{0} \rightarrow \eta_{c} K_{S}^{0}$ and $B^{+} \rightarrow \eta_{c} K^{+}$where $\eta_{c} \rightarrow K^{+} K^{-} \pi^{0}$ and $\eta_{c} \rightarrow K_{S}^{0} K^{+} \pi^{-}$using the BABAR detector. This Chapter describes the data sample used to make the branching fraction measurement, and how the $B \rightarrow \eta_{c} K$ candidates are reconstructed from the basic quantities measured in the BABAR detector. The selection procedure described here was originally optimised for a previous $B A B A R B \rightarrow \eta_{c} K$ branching 
fraction measurement [37] (henceforth referred to as the Run 1 analysis), but was revised using the data samples described below.

The aim of the reconstruction and selection procedure is to identify $B \rightarrow \eta_{c} K$ candidates from the BABAR data sample, amongst the large amount of other physics processes and detector background. The main source of background in $B \rightarrow \eta_{c} K$ selection is combinatorial background from hadronic events. Hadronic events include either $B \bar{B}$ events or light-quark and charm-quark events (so-called continuum events), $e^{+} e^{-} \rightarrow u \bar{u}, d \bar{d}, s \bar{s}, c \bar{c}$, and contain multiple hadrons and $\pi^{0}$ mesons in their final states.

Since there are several hadrons in the final states of the $B \rightarrow \eta_{c} K, \eta_{c} \rightarrow K \bar{K} \pi$, combinatorial background is more of a problem than in other exclusive charmonium channels (such as $B \rightarrow J / \Psi K, J / \psi \rightarrow l^{+} l^{-}$). Therefore particle identification and multivariate techniques are important tools in background suppression.

This Chapter describes three stages of $B \rightarrow \eta_{c} K$ candidate selection: preselection, reconstruction, and final selection. Preselection is performed to isolate potential $B \rightarrow \eta_{c} K$ events from the huge number of events in the BABAR data sample. The reconstruction stage builds a basic set of $B \rightarrow \eta_{c} K$ candidates with some kinematic constraints and loose selections applied. The final selection stage consists of choosing several discriminating variables which can be used to separate real $B \rightarrow$ $\eta_{c} K$ candidates from background, and optimising the selection criteria. The goal of the optimisation is to minimize the final error on the measured branching fraction. 


\subsection{Data Sample}

\subsubsection{Real Event Data}

The analyses used a sample of data collected by the BABAR detector between October 1999 and July 2002, which had passed quality assessment by the data collection and processing system.

The total amount of data collected at the $\Upsilon(4 S)$ resonance (called on-resonance data) corresponds to an integrated luminosity of $81 \mathrm{fb}^{-1}$ (approximately 89 million $B \bar{B}$ pairs).

The selection optimisation described here used the full $81 \mathrm{fb}^{-1}$ sample. Subsequently, some sections of the data were found to be of poor quality (due primarily to initially unnoticed problems affecting the reconstruction of EMC clusters in data processing). Thus the sample used to obtain final results (Chapter 5) was slightly smaller $\left(79.4 \mathrm{fb}^{-1}\right)$. In addition $2.5 \mathrm{fb}^{-1}$ of off-resonance (below the $\Upsilon(4 S)$ threshold) data was used to study continuum background in the development of multivariate selection (see Section 4.5.4).

\subsubsection{Monte Carlo}

Detailed Monte Carlo simulation, subject to a full detector simulation and reconstruction, was used to optimise selections and measure $B \rightarrow \eta_{c} K$ selection efficiencies. Monte Carlo production at BABAR is organised by a central Simulation Working Group. The month-by-month detector conditions are used as input to the Monte Carlo production process. The production takes place (approximately) yearly; the Monte Carlo used in this analysis is called Simulation Production 4 or 
SP4.

In the samples used, the generated Monte Carlo quantities are preserved in addition to the reconstructed ones. The reconstructed particles are associated with the generated ones by truth-matching, which enables reconstructed quantities corresponding to generated particles to be examined in the absence of background. When such an association exists for a reconstructed particle candidate, it is said to be truth-matched.

Signal Monte Carlo samples (simulated $B \bar{B}$ events where one $B$ in each event decays to $\eta_{c} K$ ) were used (see Table 4.1). Some procedures were applied to the Monte Carlo during reconstruction to correct inaccurately simulated quantities (see Section $5.3)$.

Table 4.1: Size of signal Monte Carlo samples used in this analysis.

\begin{tabular}{c|c|c|c|c}
\hline$\eta_{c}$ Decay Channel & \multicolumn{2}{|c|}{$K_{S}^{0} K^{+} \pi^{-}$} & \multicolumn{2}{c}{$K^{+} K^{-} \pi^{0}$} \\
\cline { 2 - 5 } & $B^{0}$ & $B^{+}$ & $B^{0}$ & $B^{+}$ \\
\hline \hline \# Generated Events $\left(10^{3}\right)$ & 46 & 42 & 46 & 42 \\
\hline
\end{tabular}

At the later stage of the analysis a fast Monte Carlo was used; this is described in Chapter 5. More details of the fast Monte Carlo and SP4 can be found in Appendix E.

\subsection{Preselection}

Events from the data were preselected to reduce the total amount of full reconstruction required on the full data sample. The preselected events were assigned a tagbit (see Section 2.10) which identified the events had satisfied loose criteria which 
indicated a potential $B \rightarrow \eta_{c} K$ event.

The preselection consisted of a reconstruction procedure somewhat simpler than the full reconstruction. Basic event level selections were first applied, including the requirement that the event satisfied multihadronic criteria (see Section 3.3.2). In addition, only events satisfying the following criteria were accepted: the ratio of the second to the first Fox-Wolfram moments (see Appendix D) less than 0.6; total deposited energy greater than $2.0 \mathrm{GeV}$; at least four tracks of the lowest quality (from the ChargedTracks list, see Section 2.10.2); at least three tracks of higher quality; and at least two tracks of yet higher quality.

Standard particle lists (such as the ChargedTracks list, $\pi^{0}$ list, $K_{S}^{0}$ list) were used to build $\eta_{c}$ and $B$ candidates (see Section 2.10.2). The $\eta_{c}$ candidates were required to have a reconstructed invariant mass between $2.7 \mathrm{GeV} / c^{2}$ and $3.3 \mathrm{GeV} / c^{2}$. The accompanying kaon daughter of the $B$ (henceforth referred to as the fast kaon) was required to have a centre-of-mass momentum of at least $1.5 \mathrm{GeV} / c$. Fast charged kaon candidates were required to pass loose kaon identification criteria (see Section 4.5.5), while fast $K_{S}^{0}$ candidates were required to have an invariant mass within $25 \mathrm{MeV} / c^{2}$ of the nominal $K_{S}^{0}$ mass.

Separate tagbits were defined for the $\eta_{c} \rightarrow K^{+} K^{-} \pi^{0}$ and $\eta_{c} \rightarrow K_{S}^{0} K^{+} \pi^{-}$channels. The logical combination of these tagbits was called the EtacExcl stream or EtacExcl skim, and recorded for every event in the BABAR database. The full reconstruction could then be run only on events belonging to the EtacExcl stream. 


\subsection{Reconstruction}

The full reconstruction of the $B \rightarrow \eta_{c} K$ candidates used the software packages CompositionTools, CompositionSequences and CharmUser [38] [39]. These are examples of the flexible, general purpose reconstruction tools mentioned in Section 2.10 .2 .

These tools allow $B \rightarrow \eta_{c} K$ candidates to be reconstructed from the final states using vertex and mass constraints to build the composite candidates in the decay chain. Details of the use of kinematic constraints in reconstruction at BABAR can be found in [40]. The output of the reconstruction procedure is an ntuple which forms the basic data set on which to run final selection. The ntuple contains several measured quantities for each composite and final state particle, and preserves the mother-daughter relationships between the candidates.

The reconstruction procedure involved applying some selections. Some of the selections mirrored those applied at preselection level, others relied on more sophisticated discriminating variables which will be described later in the chapter.

\section{Reconstruction of the $\pi^{0}$}

$\pi^{0} \rightarrow \gamma \gamma$ candidates were reconstructed. The photon candidates were taken to be single EMC bumps not matched to any track, with a minimum energy of $30 \mathrm{MeV}$ and a maximum lateral moment (see Section 4.5.7) of 0.8. The $\pi^{0}$ candidates were required to have a minimum energy of $0.2 \mathrm{GeV}$ and an invariant mass between $0.1 \mathrm{GeV} / c^{2}$ and $0.6 \mathrm{GeV} / c^{2}$. For $\pi^{0}$ candidates which survived, a $\pi^{0}$ mass constraint was applied to the $\gamma \gamma$ combination.

\section{Reconstruction of the $K_{S}^{0}$}


$K_{S}^{0} \rightarrow \pi^{+} \pi^{-}$candidates were reconstructed. The pion candidates were tracks taken from the ChargedTracks list. Oppositely charged tracks were combined, assigning the pion mass. A vertex constraint constraint was applied to the $\pi^{+} \pi^{-}$combination; if the vertex fit failed, four-momentum addition was used. Reconstructed $K_{S}^{0}$ candidates within $25 \mathrm{MeV} / c^{2}$ of the nominal $K_{S}^{0}$ mass were accepted. Finally a $K_{S}^{0}$ mass constraint was applied to the surviving $\pi^{+} \pi^{-}$combinations.

\section{Reconstruction of the $\eta_{c}$}

$\eta_{c} \rightarrow K^{+} K^{-} \pi^{0}$ and $\eta_{c} \rightarrow K_{S}^{0} K^{+} \pi^{-}$candidates were reconstructed. The charged kaon candidates were taken from the ChargedTracks list, and additional track quality criteria were applied. These included the requirements that the each track had a momentum of less than $10 \mathrm{GeV} / c$, and a maximum distance of closest approach to the interaction point of $1.5 \mathrm{~cm}$ in the $x, y$ plane and $10 \mathrm{~cm}$ in the $z$ direction. For $\eta_{c} \rightarrow K^{+} K^{-} \pi^{0}$ candidates, at least one charged kaon was required to pass a loose level of neural network kaon identification (see Section 4.5.5). For $\eta_{c} \rightarrow K_{S}^{0} K^{+} \pi^{-}$ candidates, the cosine of the angle between the direction of the $K_{S}^{0}$ and its momentum (see Section 4.5.6) was required to be greater than 0.90 .

A vertex constraint was applied to the $K \bar{K} \pi$ combination but 4-momentum addition was used if the fit failed. $\eta_{c}$ candidates with reconstructed masses between $2.7 \mathrm{GeV} / c^{2}$ and $3.3 \mathrm{GeV} / c^{2}$ were accepted.

\section{Reconstruction of the $B$}

$B^{0} \rightarrow \eta_{c} K_{S}^{0}$ and $B^{+} \rightarrow \eta_{c} K^{+}$candidates were reconstructed. Further criteria were applied to the fast kaon candidates, in addition to those mentioned previously. For fast charged kaons, at least 12 DCH hits were required, and only tracks with a transverse momentum of at least $0.1 \mathrm{GeV} / c$ were retained; in addition, a loose level of neural network kaon identification was imposed. For fast $K_{S}^{0}$ candidates, the 
cosine of the angle between the direction of the $K_{S}^{0}$ and its momentum was required to be greater than 0.99. All fast kaon candidates were required to have a centre-ofmass momentum greater than $1.5 \mathrm{GeV} / c$, as in the preselection.

A vertex constraint was applied to the $\eta_{c} K$ combination, and only $B$ candidates satisfying $5.15 \mathrm{GeV} / c^{2}<M_{E S}<5.30 \mathrm{GeV} / c^{2},|\Delta E|<0.25 \mathrm{GeV}$ (see Section 4.5.1) were retained. If the vertex fit failed, four-momentum addition was used.

\subsection{Candidate Selection and Optimisation}

\subsubsection{Overview of $B \rightarrow \eta_{c} K$ Selection}

The final selections applied to $B \rightarrow \eta_{c} K$ candidates were optimised by a detailed study of signal and background distributions in each selection variable.

Before describing the optimisation procedure, it is necessary to define the variables by which signal and background $B$ candidates are distinguished. In common with most other $B A B A R$ analysis $B$ candidates were selected by two largely uncorrelated variables, which use the well known kinematic quantities of the initial state $e^{+} e^{-}$collision. The first variable is the beam-energy-substituted mass and can be calculated as

$$
M_{E S}=\sqrt{\left(s / 2+\boldsymbol{p}_{U p s} \cdot \boldsymbol{p}_{B}\right)^{2} / E_{U p s}^{2}-\boldsymbol{p}_{B}^{2}},
$$

where $E_{U p s}$ is total beam energy, $\sqrt{s}$ is centre-of-mass beam energy, and $E_{B}, \boldsymbol{p}_{B}$ are the energy and momentum of the $B$ respectively. The second variable is the difference between the $B$ energy and half the beam energy, and is given by

$$
\Delta E=\left(2\left(E_{U p s} E_{B}-\boldsymbol{p}_{U p s} \cdot \boldsymbol{p}_{B}\right)-s\right) / 2 \sqrt{s},
$$

where $\boldsymbol{p}_{U p s}$ is the total momentum of the beam. 
These variables become more transparent when calculated in centre-of-mass frame, where they are given by

$$
\begin{aligned}
M_{E S} & =\sqrt{E_{\text {beam }}^{* 2}-p_{B}^{* 2}}, \\
\Delta E & =E_{B}^{*}-E_{\text {beam }}^{*},
\end{aligned}
$$

where $E_{\text {beam }}^{*}=\sqrt{s} / 2$, and $E_{B}^{*}, p_{B}^{*}$ are the energy and momentum of the $B$ in the centre-of-mass frame respectively. The resolution of $M_{E S}$ is dominated by the error on the beam energy, while the $\Delta E$ resolution is limited by the $B$ momentum measurement.

The $\Delta E, M_{E S}$ distributions in signal Monte Carlo and data are illustrated by Figures 4.1 and 4.2. Signal candidates accumulate at $\Delta E=0, M_{E S}=m_{B}$, where $m_{B}$ is the nominal value of the $B$ mass. Therefore $B \rightarrow \eta_{c} K$ candidates in the $\Delta E$ and $M_{E S}$ sidebands can be used to study combinatorial background using the full data sample.

Using the data, combinatorial background is found to be approximately linear in $\Delta E$, and is described by the empirical ARGUS function [41] in $M_{E S}$. The ARGUS function has the form

$$
A\left(M_{E S}\right) \propto\left(M_{E S} \sqrt{1-\left(M_{E S} / M_{0}\right)^{2}}\right) \exp \left[\xi\left(1-\left(M_{E S} / M_{0}\right)^{2}\right)\right],
$$

where $\xi$ and $M_{0}$ parametrize the ARGUS function shape and endpoint respectively. $M_{0}$ is fixed to half the beam energy in the center-of-mass frame $(5.290 \mathrm{GeV})$ throughout this analysis.

The separation of the $\Delta E, M_{E S}$ plane into signal and sideband regions is the fundamental method to distinguish signal and background $B \rightarrow \eta_{c} K$ candidates respectively. The optimisation of all other selection variables relies on this distinction. 

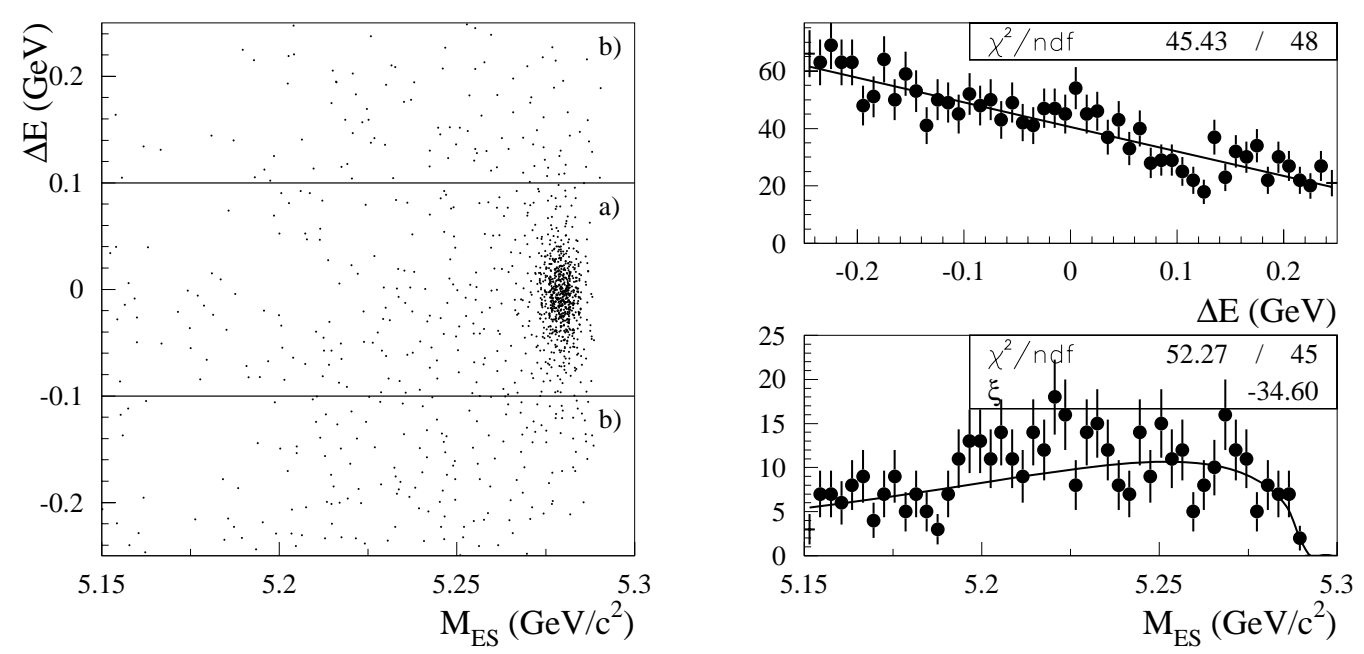

Figure 4.1: $\Delta E$ and $M_{E S}$ distributions for $B^{+} \rightarrow \eta_{c} K^{+}, \eta_{c} \rightarrow K^{+} K^{-} \pi^{0}$ candidates in signal Monte Carlo (left) and data (right), after all selections are applied. For background estimation purposes, the $\Delta E$ projection is divided into a) signal band b) sidebands. In the data distributions, $\Delta E$ is plotted for candidates with $M_{E S}<5.267 \mathrm{GeV} / \mathrm{c}^{2}, M_{E S}$ is plotted for candidates occupying the $|\Delta E|>0.1 \mathrm{GeV}$ sidebands. Linear and ARGUS function fits are superimposed respectively on the $\Delta E$ and $M_{E S}$ distributions.

\subsubsection{Selection Optimisation}

The following sections describe an optimisation of the selection for the $B \rightarrow \eta_{c} K$, $\eta_{c} \rightarrow K^{+} K^{-} \pi^{0}$ decay channels. A similar optimisation procedure was carried out in a parallel analysis for the $B \rightarrow \eta_{c} K, \eta_{c} \rightarrow K_{S}^{0} K^{+} \pi^{-}$channels [42]. The set of selection variables is described in the following sections.

The set of discriminating variables was optimised to obtain the maximum value of the sensitivity,

$$
\frac{N_{S}}{\sqrt{\left(N_{S}+N_{B}\right)}}
$$

where $N_{S}$ and $N_{B}$ are the estimated signal and background yields respectively for 
a particular set of selection criteria. The sensitivity is inversely proportional to the error on the measured branching fraction. An initial set of loose criteria was assumed as the starting point of the optimisation; for example, the initial size of the signal region was determined from the truth-matched $\Delta E, M_{E S}$ resolutions in the Monte Carlo (see Figure 4.2).
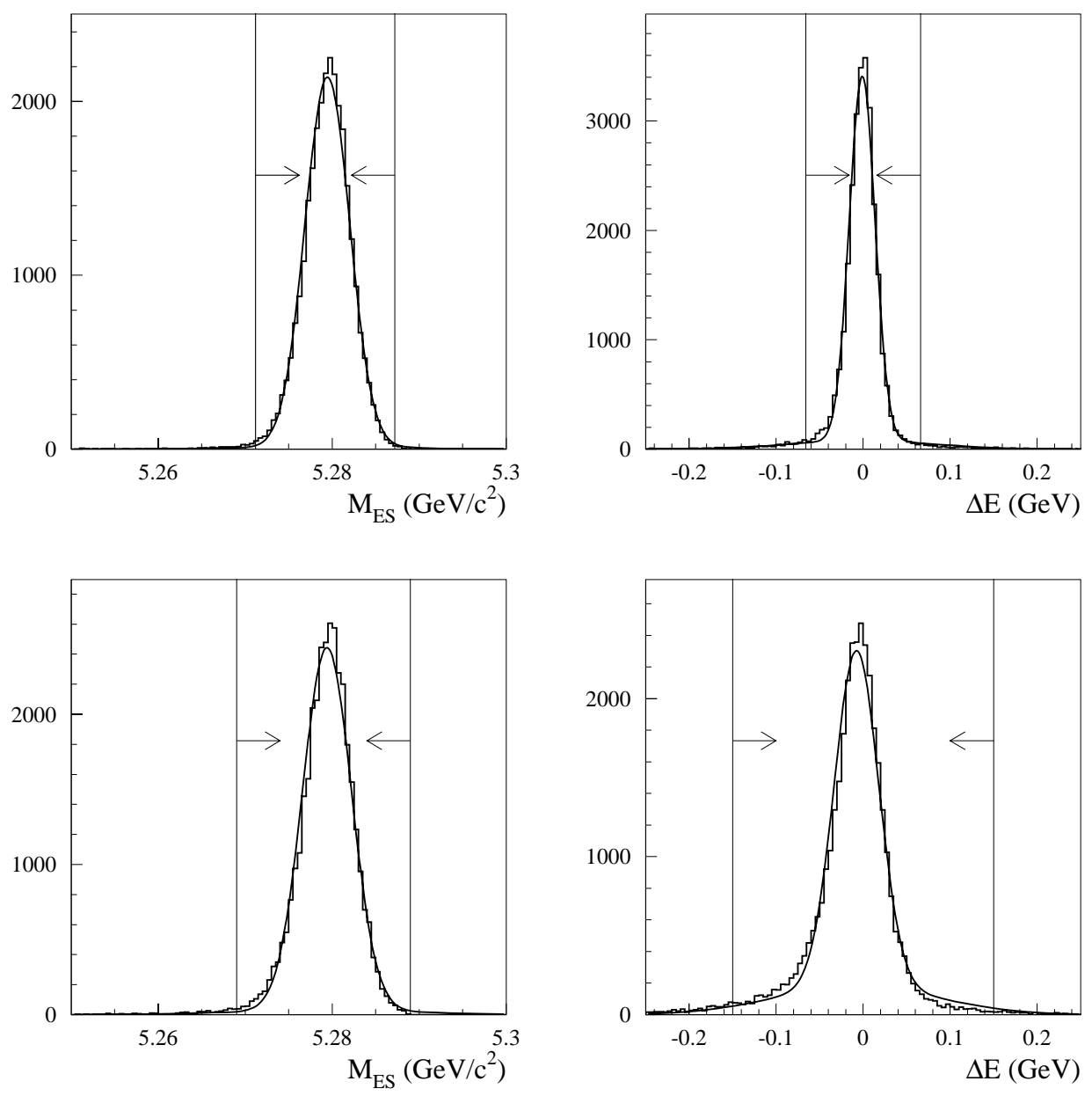

Figure 4.2: $M_{E S}$ and $\Delta E$ distributions in signal Monte Carlo for $B \rightarrow \eta_{c} K$, $\eta_{c} \rightarrow K_{S}^{0} K^{+} \pi^{-}$(top) $\eta_{c} \rightarrow K^{+} K^{-} \pi^{0}$ (bottom). The shapes are approximated by a sum of two Gaussians with normalisations $A_{1}, A_{2}$, widths $\sigma_{1}, \sigma_{2}$, and a common mean. The arrows indicate the signal region used at the beginning of the optimisation procedure. 
The selections were then optimised by an iterative procedure. At the end of the each iteration new optimal selection values were obtained and applied in the next iteration, constantly increasing the sensitivity. The iterations were repeated until the optimal selection values became stable. In this way, correlations between the selection variables are taken into account. An optimal set of selection values was first found for the $B^{0} \rightarrow \eta_{c} K_{S}^{0}$ mode, which was then used as the starting point in the optimisation for the $B^{+} \rightarrow \eta_{c} K^{+}$mode.

In each iteration $N_{S}$ was estimated using signal candidates from signal Monte Carlo while $N_{B}$ was estimated using background candidates from the full data sample. $N_{S}$ was not estimated from the data since signal yields in the $B \rightarrow \eta_{c} K$ decays are statistically limited and prone to fluctuations when adjusting selections. This follows the philosophy of a so-called blind analysis, where the signal yield in data is hidden throughout the optimisation procedure.

Signal candidates were defined as truth-matched Monte Carlo candidates within the $\Delta E, M_{E S}$ signal region after all selections. The signal region yield $\left(N_{S R}\right)$ scaled by the ratio of the number of signal events expected in the full data sample ${ }^{1}$ to the number of generated Monte Carlo events, gives an estimate of $N_{S}$.

Background candidates were defined as those occupying the $\Delta E$ sidebands in the full data sample applying the same selections. The sideband yield $\left(N_{S B}\right)$ is extrapolated to the background yield in the $\Delta E, M_{E S}$ signal region using the ARGUS function description of the $M_{E S}$ distribution and the linearity of $\Delta E$. The extrapolation is given by

$$
N_{B}=N_{S B} \cdot R_{A} \cdot R_{\Delta E}
$$

where $R_{A}$ is the ratio of the ARGUS integral in the $M_{E S}$ signal region to $N_{S B}$, and $R_{\Delta E}$ is the ratio of the width of the $\Delta E$ signal band to the $\Delta E$ sidebands.

\footnotetext{
${ }^{1}$ Using the measured branching fractions for $B \rightarrow \eta_{c} K, \eta_{c} \rightarrow K \bar{K} \pi$ from the BABAR Run1 analysis [37].
} 

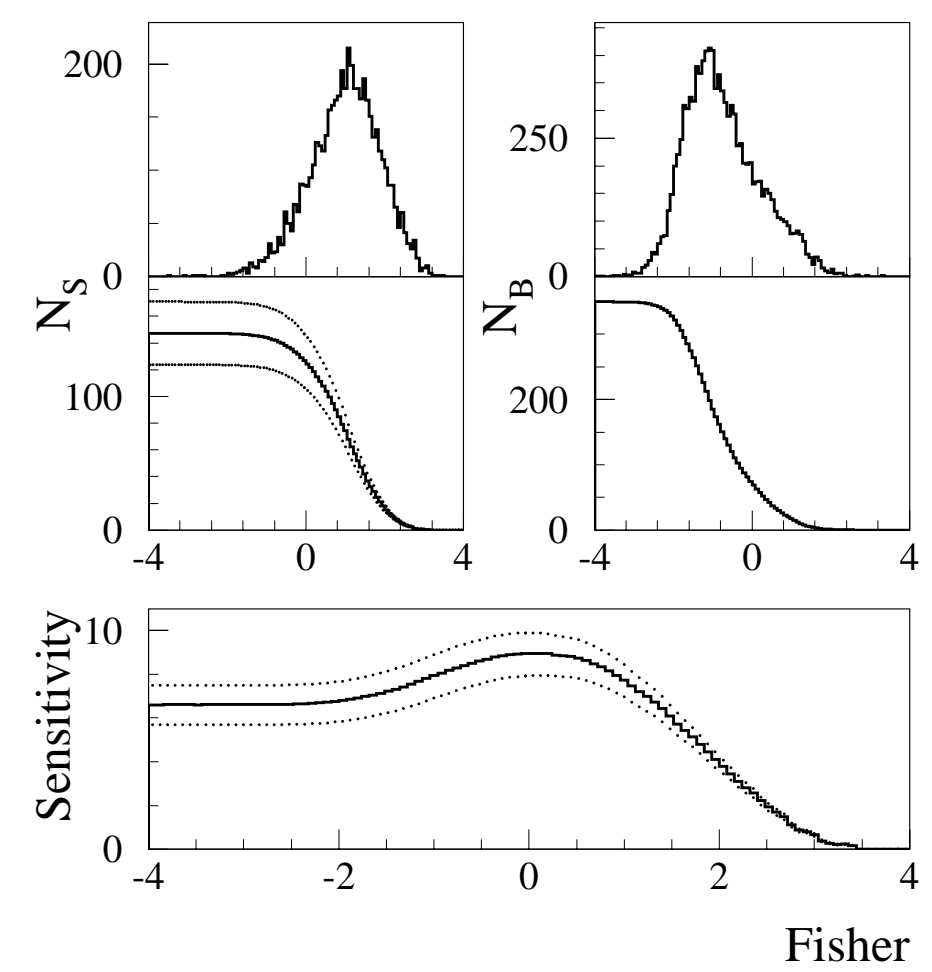

Figure 4.3: Optimisation example for the 'Fisher discriminant' variable for $B^{+} \rightarrow \eta_{c} K^{+}$. The Fisher discriminant distributions are plotted for signal and background candidates, along with $N_{S}$ and $N_{B}$ as a function of the lower limit obtained from the right-to-left integrals of the Fisher distributions (top). The resulting sensitivity $N_{S} / \sqrt{N_{S}+N_{B}}$ is also shown (bottom). The dotted lines in the $N_{S}$ and the sensitivity represent the $\pm 1 \sigma$ values of these quantities, arising from the error on the $B \rightarrow \eta_{c} K$ branching fractions.

The optimisation of a given selection variable in a given iteration was as follows. The distributions of the variable for signal and background candidates, applying all other selections were plotted; $R_{A}$ was measured from the $M_{E S}$ distribution of the background candidates. The left-to-right (right-to-left) integrated variable distributions gave $N_{S R}$ and $N_{S B}$ as a function of the lower (upper) limit imposed on the selection variable. $N_{S}, N_{B}$, and thus the sensitivity as a function of the selection limit were then deduced from the integration distributions using the scaling and 
extrapolation assumptions described above. As an example, the optimisation of the 'Fisher discriminant' selection in shown in Figure 4.3. The optimal selection values for all selection variables in a given iteration were determined by their corresponding sensitivity distributions. Thus each selection variable was optimised independently but correlations with other selection variables were taken into account by the iterative procedure.

The error on the estimated sensitivity is dominated by the estimate of $N_{S}$, which relies on the $B \rightarrow \eta_{c} K$ branching fraction measurements. This error was taken into account in the optimisation by plotting the sensitivity distributions using the central and $\pm 1 \sigma$ values of the branching fraction.

The next sections describe each selection variable and illustrate the corresponding limits found from the optimisation procedure.

\subsubsection{Two-Body Decay Kinematics Selection}

The kinematic quantities of two-body decays such as $B \rightarrow \eta_{c} K$ are constrained by energy and momentum conservation. The momenta of the two daughter particles in the rest frame of the mother particle are determined by the masses of the three particles involved.

A useful quantity is $\left(\cos \theta_{H}\right)^{B}$, the so-called helicity angle of the $B$, defined as the angle between the kaon momentum in the $B$ rest frame and the $B$ momentum in the $\Upsilon(4 S)$ rest frame. $\left(\cos \theta_{H}\right)^{B}$ may be deduced from the measured energy of the fast kaon candidate, using a two-body decay hypothesis. $B \rightarrow \eta_{c} K$ events should have $\left|\left(\cos \theta_{H}\right)^{B}\right|<1$, within experimental resolution.

This calculation of $\left(\cos \theta_{H}\right)^{B}$ using the two-body hypothesis can be performed by 
considering the Lorentz transformation of the kaon energy $E_{K}^{B}$ from the $B$ frame to the $\Upsilon(4 S)$ frame

$$
E_{K}^{*}=\gamma_{B}\left(E_{K}^{B}-\beta_{B} p_{K}^{\|}\right),
$$

where $E_{K}^{*}$ is the kaon momentum in the $\Upsilon(4 S)$ frame, $\beta_{B}$ is the relative speed between the $B$ and $\Upsilon(4 S)$ frames and $\gamma_{B}$ is the corresponding Lorentz boost factor. $p_{K}^{\|}$is the component of the kaon momentum in the boost direction and is given by $\left|\boldsymbol{p}_{K}^{B}\right| \times\left(\cos \theta_{H}\right)^{B}$. Thus,

$$
\left(\cos \theta_{H}\right)^{B}=\frac{E_{K}^{B}-E_{K}^{*} / \gamma_{B}}{\beta_{B}\left|\boldsymbol{p}_{K}^{B}\right|} .
$$

The two body hypothesis $B \rightarrow \eta_{c} K$ is used to make the substitutions

$$
\begin{aligned}
\left|\boldsymbol{p}_{K}^{B}\right| & =\frac{\sqrt{\left(m_{B}+m_{K}-m_{\eta_{c}}\right)\left(m_{B}+m_{K}+m_{\eta_{c}}\right)\left(m_{B}-m_{K}-m_{\eta_{c}}\right)\left(m_{B}-m_{K}+m_{\eta_{c}}\right)}}{2 m_{B}}, \\
E_{K}^{B} & =\sqrt{m_{B}^{2}+\left|\boldsymbol{p}_{K}^{B}\right|^{2}},
\end{aligned}
$$

where $m_{B}, m_{K}$ are the nominal values of the $B$ mass and the kaon mass respectively. $m_{\eta_{c}}$ is the reconstructed value of the $\eta_{c}$ mass rather than the nominal value, which takes into account the finite width of the $\eta_{c}$.

The illustration of signal and background distributions for the $\left(\cos \theta_{H}\right)^{B}$ variable can be seen in Figure 4.4. The $\left(\cos \theta_{H}\right)^{B}$ values are not restricted to $\left|\cos _{H} \theta_{K}^{B}\right|<1$ for signal candidates due to experimental resolution.

Studies [43] showed $\left(\cos \theta_{H}\right)^{B}$ was a more useful indicator of two-body decays than the momenta of the $B$ daughters, which tend to be correlated with the reconstructed mass of the $\eta_{c} . B \rightarrow \eta_{c} K$ candidates were accepted within the range $\left|\cos _{H} \theta_{K}^{B}\right|<1.2$; this selection was not optimised. 


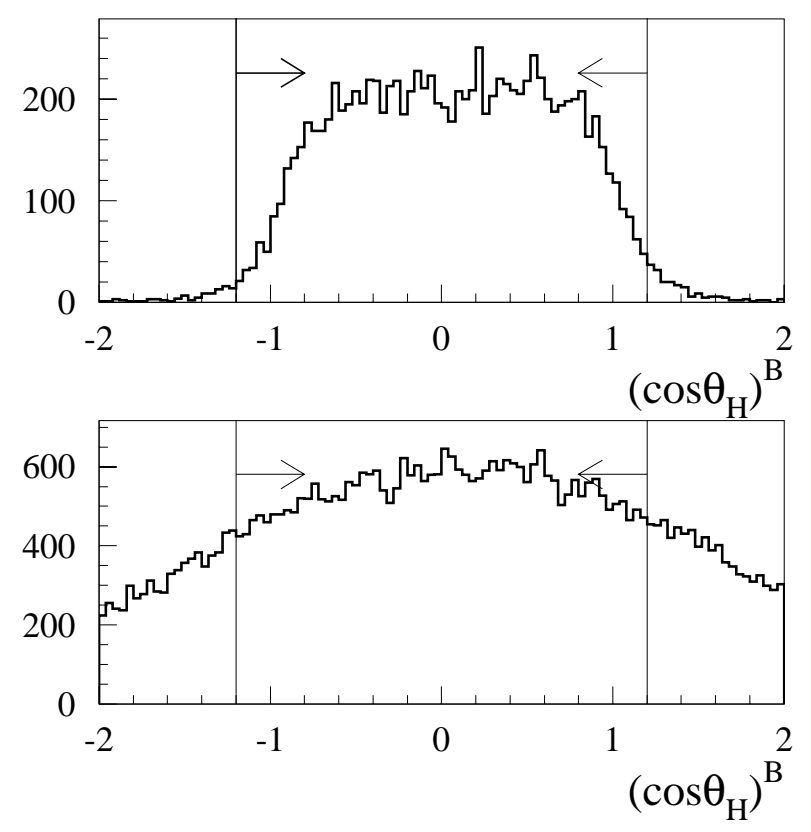

Figure 4.4: Distribution of $\left(\cos \theta_{H}\right)^{B}$ for truth-matched $B^{+} \rightarrow \eta_{c} K^{+}, \eta_{c} \rightarrow$ $K^{+} K^{-} \pi^{0}$ Monte Carlo candidates (top) and data (bottom). The data distribution is taken from the $|\Delta E|>0.1 \mathrm{GeV}$ sidebands, with no other selections applied. The arrow indicate the selected region in this analysis.

\subsubsection{Fisher Discriminant}

An important background suppression tool used for this analysis was a Fisher discriminant, a multivariate selection tool providing separation of signal $B \rightarrow \eta_{c} K$ candidates from continuum (non- $B \bar{B}$ ) background. Fisher discriminants have been used in other analyses at BABAR, particularly those involving charmless hadronic $B$ decays [44] [45], where backgrounds contamination is high compared to exclusive charmonium reconstruction. In some charmonium channels (such as $B \rightarrow J / \psi \pi^{0}$ ) where background is more problematic, a Fisher discriminant has helped enhance the selected signal purity. The combinatorial background affecting the reconstruction of hadronic $\eta_{c}$ decays is significant, and makes the use of the more powerful 
multivariate selection desirable.

The Fisher discriminant (see for example [46]) is a linear combination of the chosen set of variables which allows greater separation of the signal and background classes than is obtained by successively selecting candidates using each selection variable separately. If the set of $N$ discriminating variables is denoted by $x_{i}$, the Fisher discriminant is given by

$$
\mathcal{F}=\sum_{i}^{N} \alpha_{i} x_{i} .
$$

The separation of Fisher distributions from signal and background classes $\left(\mathcal{F}^{S}\right.$ and $\mathcal{F}^{B}$, respectively) is described by the quantity

$$
\Delta=\frac{\left\langle\mathcal{F}^{S}\right\rangle-\left\langle\mathcal{F}^{B}\right\rangle}{\sigma_{\mathcal{F}^{S}}^{2}+\sigma_{\mathcal{F}^{B}}^{2}}
$$

Differentiating $\Delta$ with respect to each variable $x_{i}$ and setting the result to zero (finding the maximum signal-background separation), yields the expressions for the coefficients up to a constant scaling factor

$$
\alpha_{i} \propto \sum_{j}^{N}\left(U_{i j}^{S}+U_{i j}^{B}\right)\left(\mu_{j}^{S}+\mu_{j}^{B}\right)
$$

where $U_{i j}^{S}, U_{i j}^{B}$ are the covariance matrices of the input variables $x_{i}$ for signal and background $B$ candidates respectively, and $\mu_{j}^{S}, \mu_{j}^{B}$ are the mean values of the input variables.

The input variables $x_{i}$ may be arbitrarily scaled transformations of raw variables, such that their means can take any desired values,

$$
x_{i}=a_{i}+b_{i} x_{i}^{\prime}
$$

where $x_{i}^{\prime}$ are the raw variables. The values of the covariance matrices $U_{i j}^{S}, U_{i j}^{B}$ are then altered accordingly. In addition, from the freedom of defining the Fisher coefficients (4.12) it follows that that the mean values of $\mathcal{F}^{S}$ and $\mathcal{F}^{B}$ can take any desired values. In this analysis, the variable scaling factors $a_{i}, b_{i}$ were chosen such 
that the mean of each variable was +1 for signal events and -1 for background events $^{2}$. The coefficients $\alpha_{i}$ were then normalised such that $\sum_{i}^{N} \alpha_{i}=1$. The corresponding Fisher distributions for signal and background events have means $\left\langle\mathcal{F}^{S}\right\rangle=1$ and $\left\langle\mathcal{F}^{B}\right\rangle=-1$ respectively.

The variables $x_{i}^{\prime}$ chosen in this analysis were based on event-shape. In $e^{+} e^{-} \rightarrow B \bar{B}$ events containing signal candidates, the final state particles tend to be spherically distributed in the center-of-mass frame. Continuum events tend to result in back-toback jets of particles. 9 event-shape variables were used in the Fisher discriminant for this analysis, which are described in Appendix D.

In addition to event-shape variables, the Fisher discriminant utilised energy flow cones, used in previous $B$ meson analyses [47]. 9 spatial cones were defined, (in the $\Upsilon(4 S)$ rest frame), dividing the polar angle with respect to the $\eta_{c}$ momentum from $0^{\circ}$ to $90^{\circ}$ into $10^{\circ}$ intervals. Each cone was reflected along the $\eta_{c}$ momentum, so as to encompass the entire solid angle. The summed charged and neutral energy of particles whose momenta lie in each of these cones (assigning the pion mass for charged particles, and excluding those particles used to reconstruct the $B$ ) gives a measure of how jet-like the event is. The 'cone energies' $\left(E_{1}\right.$ to $\left.E_{9}\right)$, for signal Monte Carlo and off-resonance data, can be seen in Figure 4.5. The cones with narrower opening angles show a higher occupation of event energy for off-resonance data than for signal Monte Carlo.

The Fisher coefficients $\alpha_{i}$ were determined using signal Monte Carlo and off-resonance data samples defined in Section 4.2. The means of each variable for signal and background samples, and the covariance matrices in (4.12) were calculated using all candidates. Only the EtacExcl stream selections were applied (which are not applied in the production of signal Monte Carlo), and a truth-match was required for

\footnotetext{
${ }^{2}$ The technical implementation of the Fisher discriminant tool, including choice of variables, was performed by G. Sciolla (Charmonium Working Group).
} 

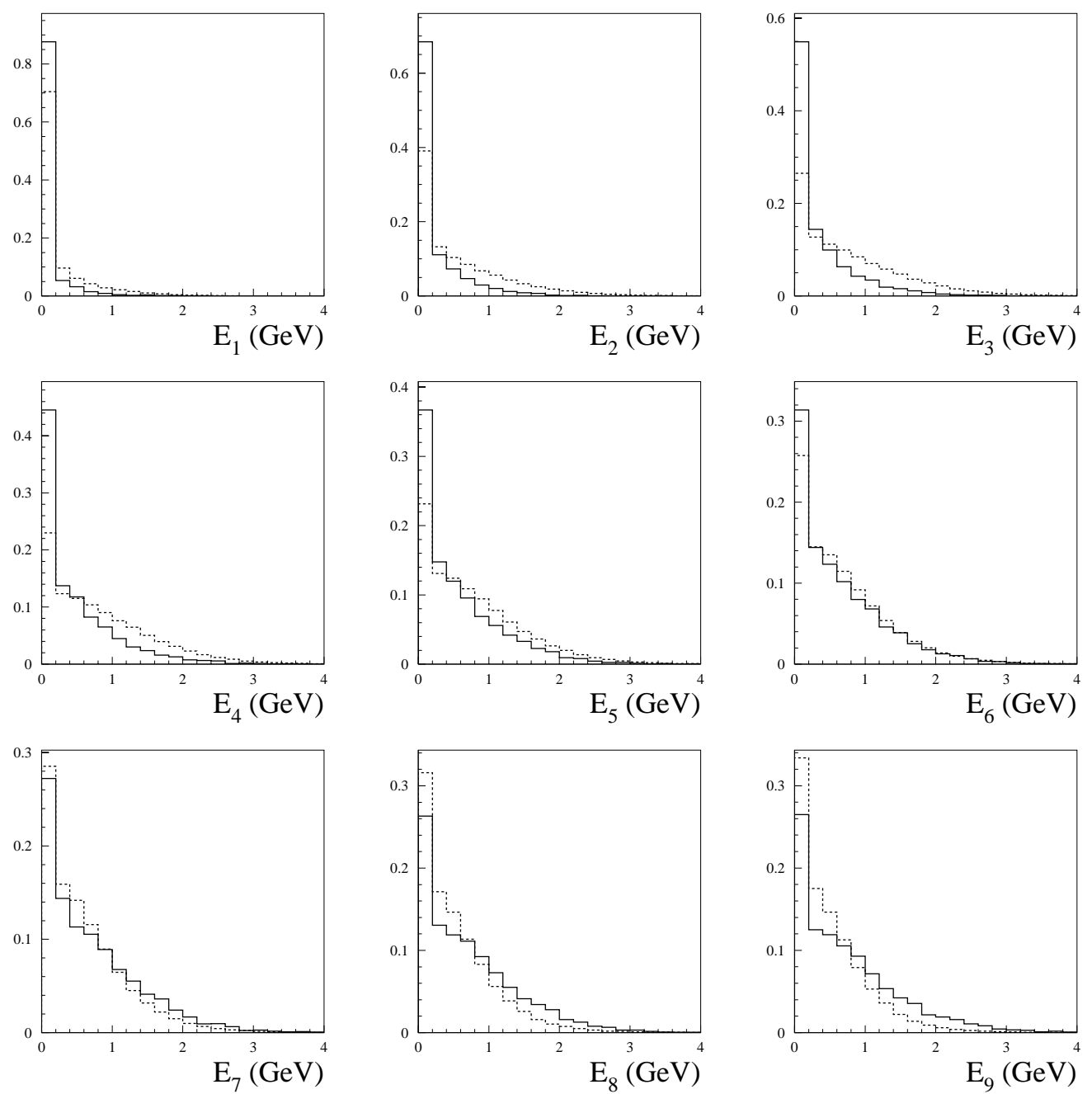

Figure 4.5: 'Cone energies' (see text) for $B \rightarrow \eta_{c} K, \eta_{c} \rightarrow K^{+} K^{-} \pi^{0}$ signal Monte Carlo (solid line) and off-resonance data (dashed line). Each cone is $10^{\circ}$ wide and the angles of the outer edges of the cones numbered 1 to 9 range from $10^{\circ}$ to $90^{\circ}$.

each simulated candidate. A separate determination of Fisher coefficients was carried out for $B \rightarrow \eta_{c} K, \eta_{c} \rightarrow K^{+} K^{-} \pi^{0}$ and $B \rightarrow \eta_{c} K, \eta_{c} \rightarrow K_{S}^{0} K^{+} \pi^{-}$candidates, combining charged and neutral $B$ decays.

The Fisher distributions for $B \rightarrow \eta_{c} K, \eta_{c} \rightarrow K^{+} K^{-} \pi^{0}$ signal Monte-Carlo and 

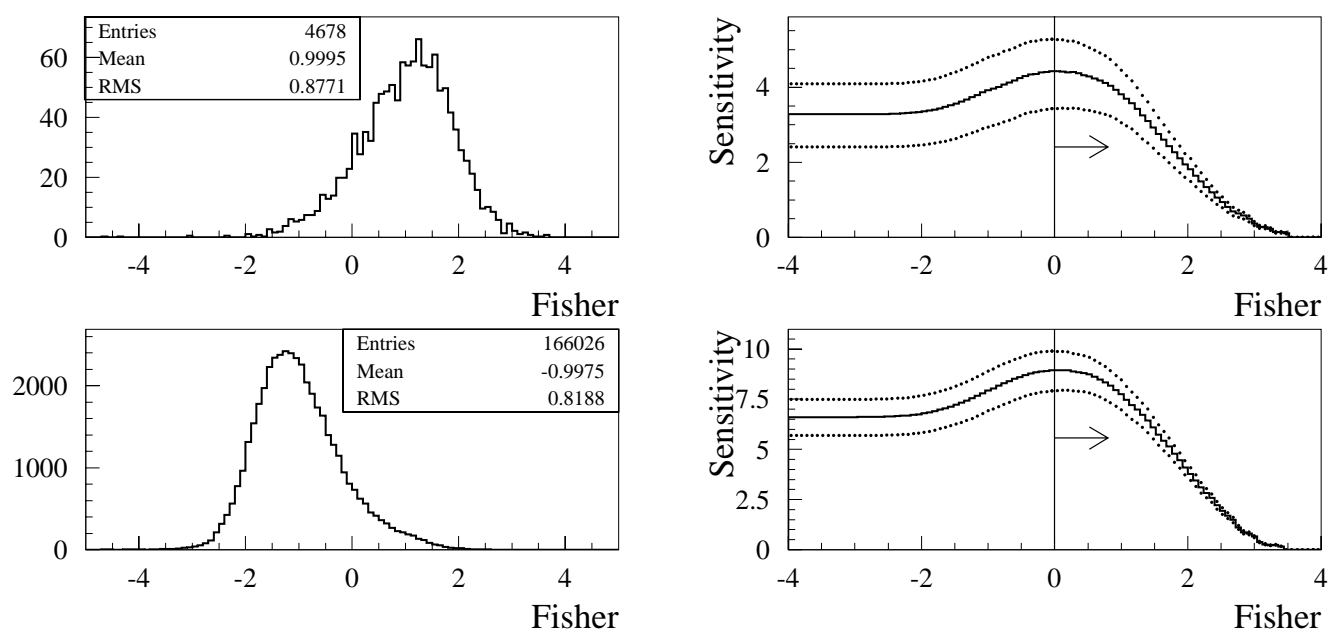

Figure 4.6: Fisher discriminant distribution for signal and background (left), and its selection optimisation (right). The Fisher discriminant is plotted for $B \rightarrow \eta_{c} K, \eta_{c} \rightarrow K^{+} K^{-} \pi^{0}$ Monte Carlo (top left) and off-resonance data (bottom left). The central (line) and $\pm 1 \sigma$ (dots) sensitivity curves obtained from the optimisation procedure are plotted, with all other selections applied, for $B^{0} \rightarrow \eta_{c} K_{S}^{0}$ (top right) and $B^{+} \rightarrow \eta_{c} K^{+}$(bottom right). The arrows denote the optimal selection cut.

off-resonance data is shown in Figure 4.6. The optimal selection for $B \rightarrow \eta_{c} K$, $\eta_{c} \rightarrow K^{+} K^{-} \pi^{0}$ was found to be $\mathcal{F}>0.0$. The optimal selection values for $B \rightarrow \eta_{c} K$, $\eta_{c} \rightarrow K_{S}^{0} K^{+} \pi^{-}$are given in Table 4.2.

\subsubsection{Charged Kaon Identification}

Another crucial tool in combinatorial background suppression is kaon identification, which uses the discriminating quantities such as $\mathrm{dE} / \mathrm{d} x$ and DIRC information described in Chapter 2. Several types of kaon selection have been developed by the Particle Identification Working Group [48] at BABAR based on the these quantities. 
Each type of kaon identification is classified by a selector.

The kaon selection in this analysis uses a neural network selector to combine the information from the SVT, DCH, and DIRC. A relative likelihood $\mathcal{L}_{K} /\left(\mathcal{L}_{\pi}+\mathcal{L}_{K}\right)$ is defined for each detector subsystem. $\mathrm{dE} / \mathrm{d} x$ measurements are used to calculate the SVT, DCH likelihoods, while the DIRC likelihood is calculated from a fit to the number of photons detected and their position and arrival times with respect to the track being identified. These likelihoods along with the track momentum are fed to the neural network, whose output is a continuous variable close to 1 for kaons and close to 0 for non-kaons. 5 discrete levels of neural network kaon identification are available for each reconstructed track: Not-a-pion (NAP), VeryLoose (VL), Loose (L), Tight (T) and VeryTight (VT), which correspond to increasing minimum selection thresholds on the neural network output.

The efficiency of the neural network selector has been measured using control samples of kaons in data, from the decay $D^{*} \rightarrow D^{0} \pi, D^{0} \rightarrow K \pi$; the rate of pions misidentified as kaons has also been measured. The performance in SP4 Monte Carlo and in data for several types of kaon selector is shown in Figure 4.7. It can be seen that the efficiency is high and and misidentification rate low over the whole range of kaon momentum, and that there is some level of discrepancy in performance in data and SP4.

Kaon identification was applied at reconstruction level to reduce the final size of the samples (see Sections 4.3 and 4.4). The optimal identification level was found to be VeryLoose for all the kaons in the $B \rightarrow \eta_{c} K, \eta_{c} \rightarrow K^{+} K^{-} \pi^{0}$ channel (see Figure 4.8). In the $B \rightarrow \eta_{c} K, \eta_{c} \rightarrow K_{S}^{0} K^{+} \pi^{-}$channel, the optimal levels were found to be Not-a-pion for the $\eta_{c}$ daughter kaon, and VeryLoose for the fast kaon. 

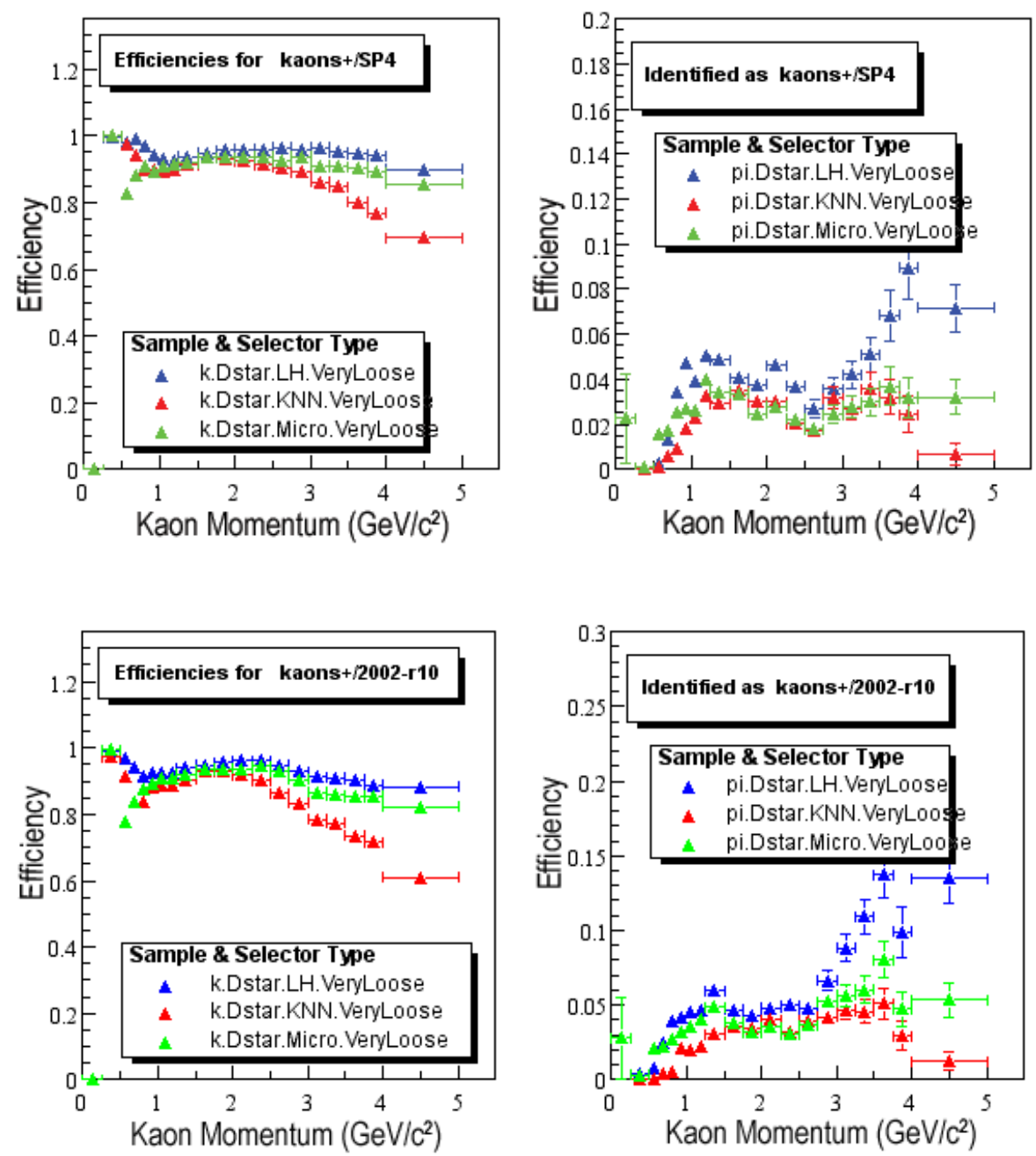

Figure 4.7: Efficiency and pion misidentification rate for kaon selectors in SP4 Monte Carlo (top) and data (bottom). The VeryLoose level of selection is shown for Likelihood ('LH'), neural net ('KNN'), and simple ('Micro') kaon selectors.

\subsection{6 $\quad K_{S}^{0}$ Selection}

The loose preselection based on the unconstrained $K_{S}^{0}$ invariant mass may be optimised to obtain the maximum sensitivity. Figure 4.9 shows the $K_{S}^{0}$ mass distribution in signal Monte Carlo and from a sample of on-resonance data. The combinatorial 

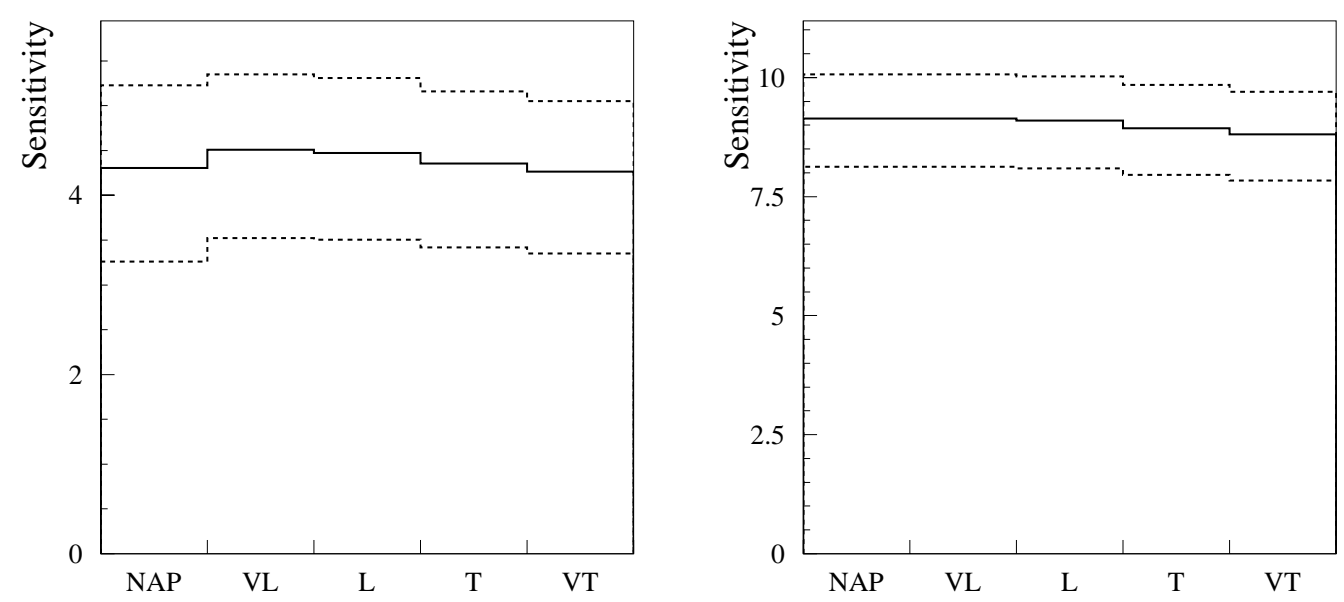

Figure 4.8: Kaon ID optimisation for the $\eta_{c}$ daughter kaons in $B^{0} \rightarrow \eta_{c} K_{S}^{0}$, $\eta_{c} \rightarrow K^{+} K^{-} \pi^{0}$ (left) and for the fast kaon in $B^{+} \rightarrow \eta_{c} K^{+}, \eta_{c} \rightarrow K^{+} K^{-} \pi^{0}$ (right). The central (line) and $\pm 1 \sigma$ (dots) sensitivity values obtained from the optimisation procedure are plotted, with all other selections applied.

background is clearly visible in the data. The optimal selection range for the $K_{S}^{0}$ mass in $B^{0} \rightarrow \eta_{c} K_{S}^{0}, \eta_{c} \rightarrow K^{+} K^{-} \pi^{0}$ was found to be within $13 \mathrm{MeV} / c^{2}$ of the nominal $K_{S}^{0}$ mass, as shown in Figure 4.9.

Two further quantities can be used to improve the purity of $K_{S}^{0}$ candidates and enhance the sensitivity. To exploit the fact that $K_{S}^{0}$ particles travel from the interaction point before decaying the $K_{S}^{0}$ flight length, normalised to its error is used as a selection variable. In addition, the cosine of the angle between the $K_{S}^{0}$ direction (defined by the vector joining the decay vertex of the $K_{S}^{0}$ and that of its mother) and the $K_{S}^{0}$ momentum, $\cos \alpha\left(K_{S}^{0}\right)$, is used as another selection variable. The flight length distribution and the $\cos \alpha\left(K_{S}^{0}\right)$ distribution from $B^{0} \rightarrow \eta_{c} K_{S}^{0}, \eta_{c} \rightarrow K^{+} K^{-} \pi^{0}$ candidates from signal Monte Carlo, compared to those from the data can be seen in Figure 4.10. A peak from fake $K_{S}^{0}$ candidates is visible in the data where the flight length is of the same magnitude as the error. The optimal selection values 

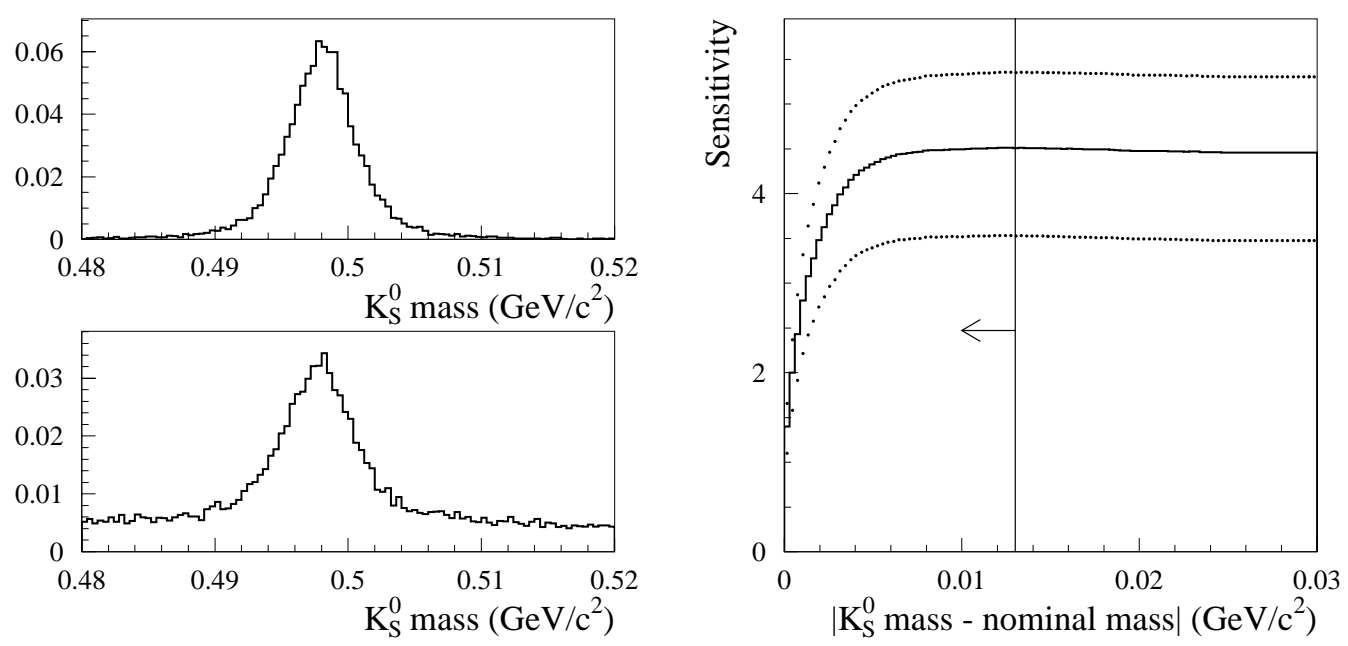

Figure 4.9: Unconstrained invariant mass distribution of $K_{S}^{0}$ candidates (left) and its selection optimisation (right) for $B^{0} \rightarrow \eta_{c} K_{S}^{0}, \eta_{c} \rightarrow K^{+} K^{-} \pi^{0}$. The $K_{S}^{0}$ mass from signal Monte Carlo (truth-matched, top left) and data $(|\Delta E|$ $>0.10 \mathrm{GeV}$, no other selections, bottom left) are shown. The central (line) and $\pm 1 \sigma$ (dots) sensitivity curves obtained from the optimisation procedure are plotted, with all other selections applied. The arrow denotes the optimal selection cut.

were found to be $>4.0$ for the normalised flight length, and $\cos \alpha\left(K_{S}^{0}\right)>0.9995$, although the sensitivity maxima are not very pronounced (see Figure 4.10). This implies the discrimination power of the two variables is not very great when all other selections are applied.

For the $B \rightarrow \eta_{c} K, \eta_{c} \rightarrow K_{S}^{0} K^{+} \pi^{-}$channel, separate optimal $K_{S}^{0}$ selection values were found for the fast $K_{S}^{0}$ and the $\eta_{c}$ daughter $K_{S}^{0}$. The optimal selection values were similar to those in the $B \rightarrow \eta_{c} K, \eta_{c} \rightarrow K^{+} K^{-} \pi^{0}$ channel, and are summarised in Table 4.2. 

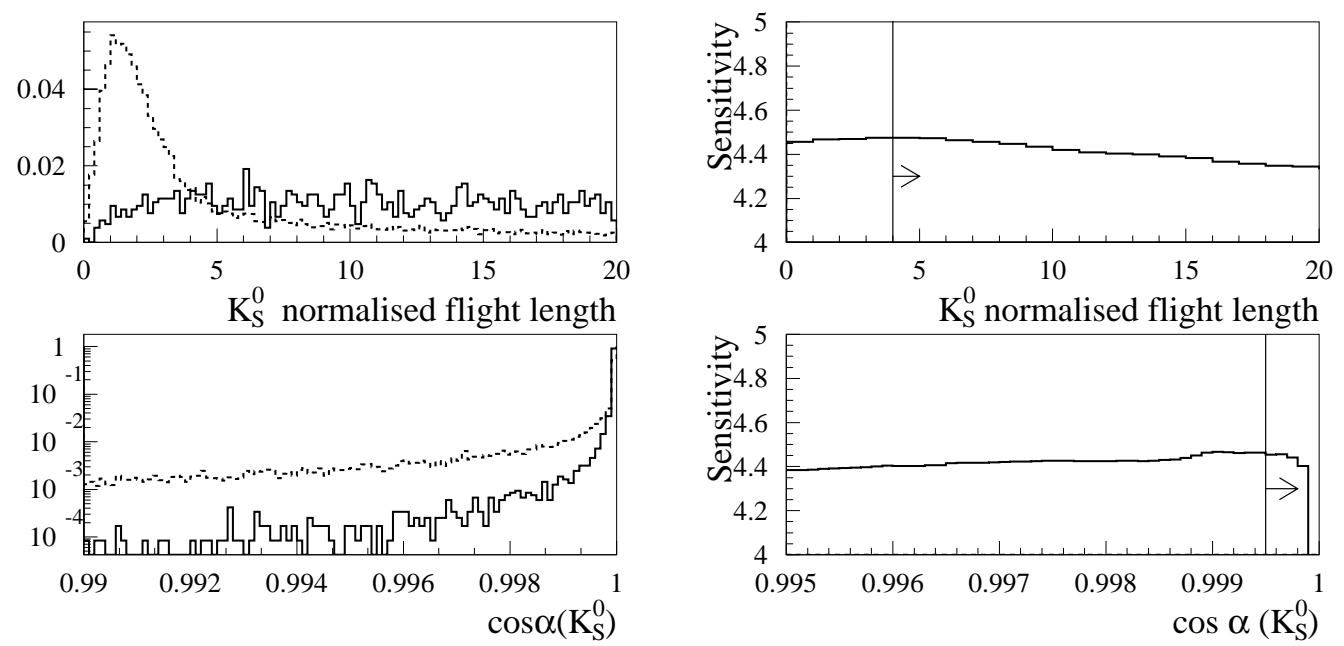

Figure 4.10: $K_{S}^{0}$ flight length (top left) and $\cos \alpha\left(K_{S}^{0}\right)$ (bottom left) and their optimisation (right) for $B^{0} \rightarrow \eta_{c} K_{S}^{0}, \eta_{c} \rightarrow K^{+} K^{-} \pi^{0}$. The quantities are shown for signal Monte Carlo (truth-matched, solid line) and data $|\Delta E|>$ $0.10 \mathrm{GeV}$, no other selections, dashed line); the histograms are normalised to unit area. The arrows denote the optimal selection cuts (all other selections applied).

\subsection{7 $\quad \pi^{0}$ Selection}

Like the $K_{S}^{0}$ candidates, the $\pi^{0}$ candidates in the $B \rightarrow \eta_{c} K, \eta_{c} \rightarrow K^{+} K^{-} \pi^{0}$ channel were selected by their unconstrained invariant mass. The $\pi^{0}$ mass distribution in signal Monte Carlo and on-resonance data can be seen in Figure 4.11. The optimal selection range for the $\pi^{0}$ mass was found to be within $16 \mathrm{MeV} / c^{2}$ of the nominal $\pi^{0}$ mass.

Other selections were also applied to the daughter photons of the $\pi^{0}$. Separate minimum energy thresholds were applied to the lower energy and higher energy photon in each pair, as in the $\pi^{0}$ selection in Chapter 3. The distributions of the photons energies in Monte Carlo and data can be seen in Figure 4.12. The optimal 

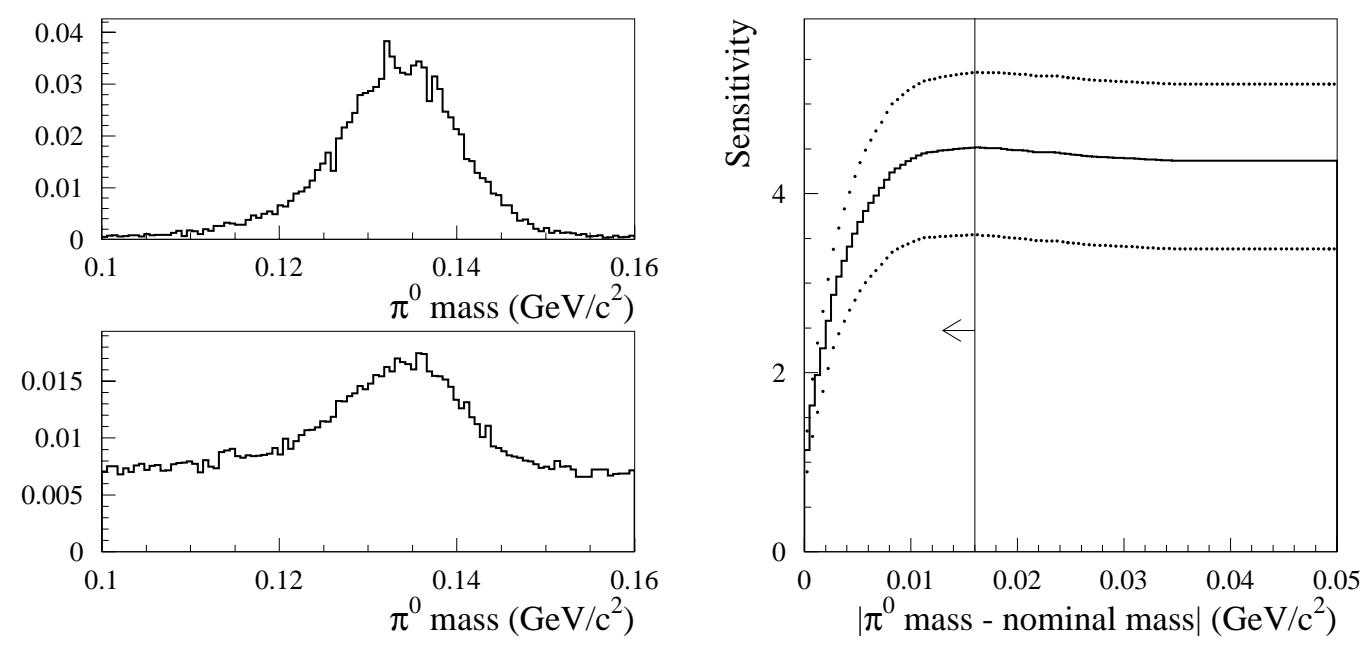

Figure 4.11: Unconstrained invariant mass distribution of $\pi^{0}$ candidates (left) and its optimisation (right), for $B^{0} \rightarrow \eta_{c} K_{S}^{0}, \eta_{c} \rightarrow K^{+} K^{-} \pi^{0}$. The $\pi^{0}$ mass from signal Monte Carlo (truth-matched, top left) and on-resonance data $(|\Delta E|>0.10$, no other selections, bottom). The central (line) and $\pm 1 \sigma$ (dots) sensitivity curves obtained from the optimisation procedure are plotted, with all other selections applied. The arrow denotes the optimal selection cut.

selection limits were found to be $E_{\gamma}>40 \mathrm{MeV}$ for the lower energy photon and $E_{\gamma}>230 \mathrm{MeV}$ for the higher energy photon.

The lateral moment (LAT) [49] of each daughter was also used, see Figure 4.13. The LAT variable is a measure of the shower shape in the calorimeter, and distinguishes electromagnetic from hadronic showers. LAT takes values between 0 and 1; low values are consistent with electromagnetic showers in which the shower energy is concentrated in a small number of crystals, higher values are consistent with hadronic showers where the energy is more evenly spread across crystals. A large number of fake photons with very low values of LAT are visible in the data. Only photons with LAT $>0.01$ were accepted. While requiring a maximum value of LAT can reduce background from hadronic showers, in the optimisation such a selection 

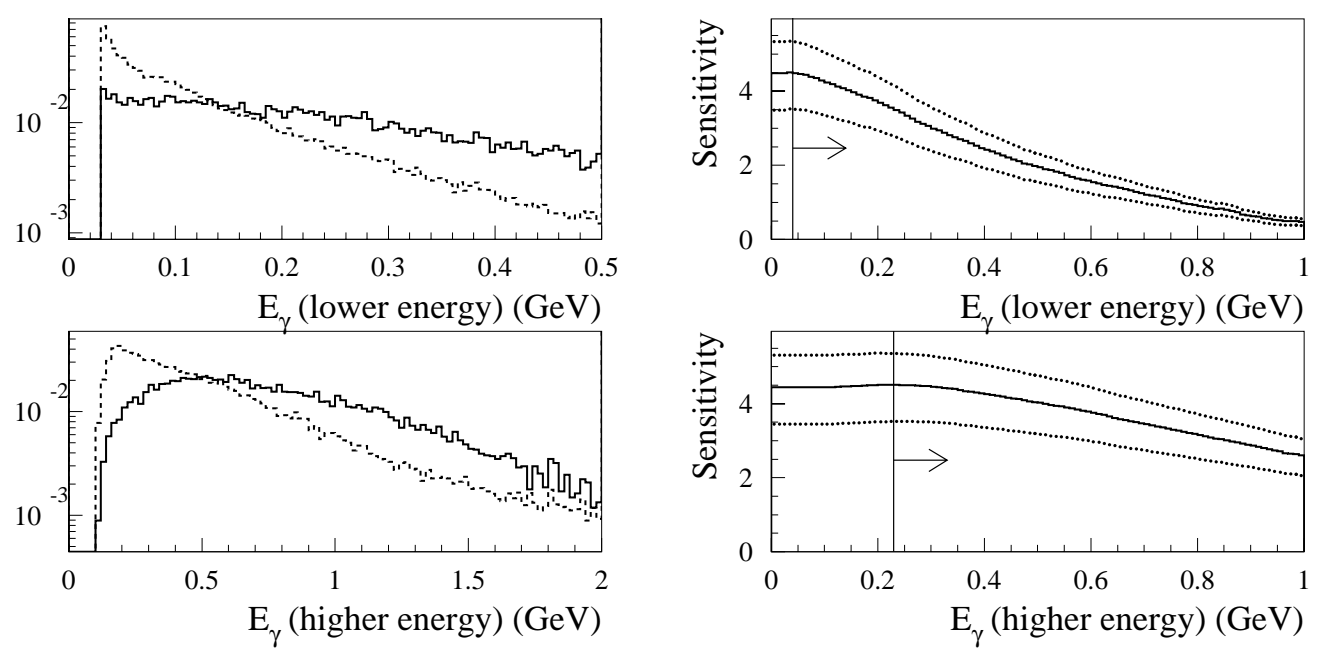

Figure 4.12: Photon energy distributions from $\pi^{0}$ candidates (left) and their selection optimisation (right) for $B^{0} \rightarrow \eta_{c} K_{S}^{0}, \eta_{c} \rightarrow K^{+} K^{-} \pi^{0}$. The photon energies from signal Monte Carlo (truth-matched, solid line) and data $(|\Delta E|$ $>0.10 \mathrm{GeV}$, no other selections, dashed line) are shown (the histograms are normalised to unit area). The central (line) and $\pm 1 \sigma$ (dots) sensitivity curves obtained from the optimisation procedure are plotted, with all other selections applied. The arrows denote the optimal selection cuts.

did not greatly influence the sensitivity. Therefore, no maximum LAT value requirement was placed on the photons. In the Run 1 analysis, the helicity angle of the $\pi^{0}$ was also used as a selection variable. The $\pi^{0}$ helicity angle, $\left|\left(\cos _{H} \theta\right)^{\pi^{0}}\right|$ is defined as the cosine of the angle between the $\pi^{0}$ momentum in the laboratory frame and the photons' momenta in the $\pi^{0}$ rest frame. Since the $\pi^{0}$ is spinless this angle should be distributed isotropically. However, a large component of the background consists of a low energy photon combined with a high energy photon which leads to an accumulation of $\pi^{0}$ candidates at $\left|\left(\cos _{H} \theta\right)^{\pi^{0}}\right| \approx 1$ (see Figure 4.13). In the optimisation procedure, it was found that applying a selection to $\left|\left(\cos _{H} \theta\right)^{\pi^{0}}\right|$ in addition to the other variables only decreased the sensitivity, and so no selection on the $\pi^{0}$ helicity was used. 

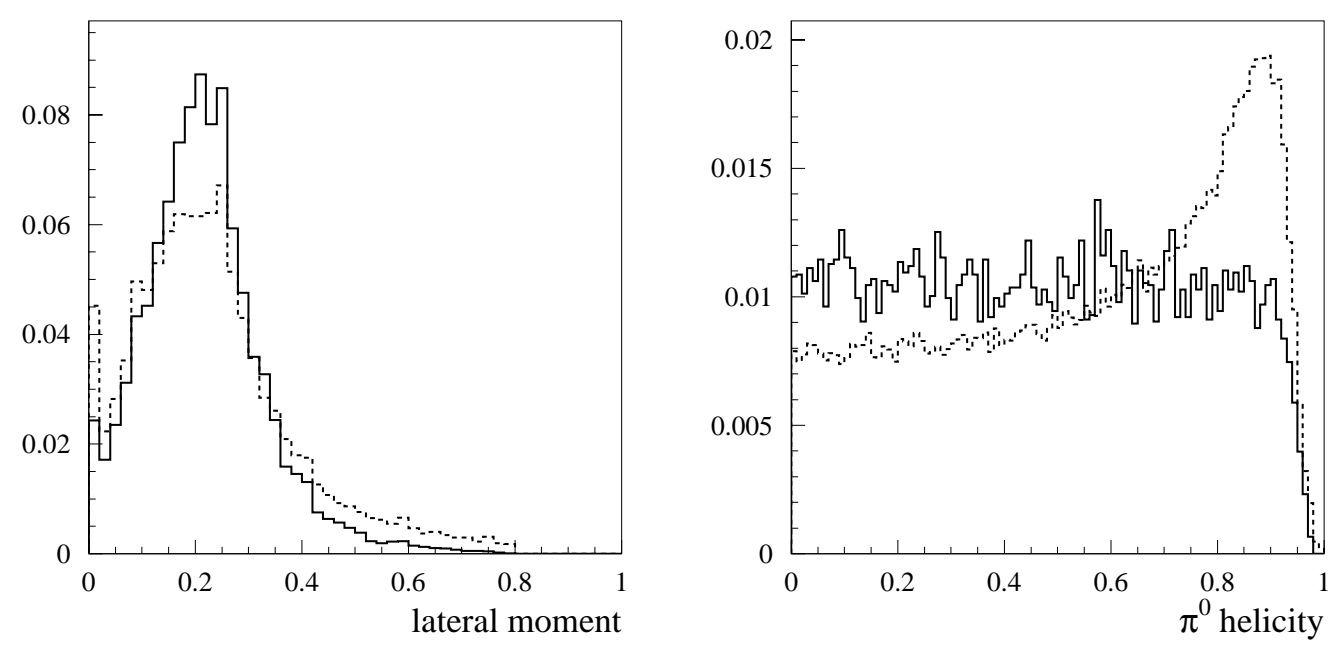

Figure 4.13: $L A T$ (left) and $\pi^{0}$ helicity (right) from $B^{0} \rightarrow \eta_{c} K_{S}^{0}, \eta_{c} \rightarrow$ $K^{+} K^{-} \pi^{0}$ candidates from signal Monte Carlo (truth-matched, solid line) and on-resonance data $(|\Delta E|>0.10 \mathrm{GeV}$, no other selections, dashed line). The histograms are normalised to unit area.

\subsection{8 $\eta_{c}$ Selection}

Since the $\eta_{c}$ meson is a broad resonance, a mass constraint was not applied to $\eta_{c}$ candidates. The $\eta_{c}$ mass distributions from truth-matched signal Monte Carlo can be seen in Figure 4.14. The simulated $\eta_{c}$ was generated with a mass width of $27.0 \mathrm{MeV} / \mathrm{c}^{2}$. The resolution distributions (reconstructed mass minus generated mass) are also shown. Double Gaussian fits to the resolution distributions yield effective widths ${ }^{3}$ of $20 \mathrm{MeV} / c^{2}$ and $10 \mathrm{MeV} / c^{2}$ for the $\eta_{c} \rightarrow K^{+} K^{-} \pi^{0}$ and $\eta_{c} \rightarrow K_{S}^{0} K^{+} \pi^{-}$ resolutions respectively. It should be noted that the measured resolution is sensitive to the level of selection applied. When selections are not applied substantially longer tails are observed in the resolution distributions.

\footnotetext{
${ }^{3}$ The double Gaussian effective width is defined as $\sqrt{w_{1} \sigma_{1}^{2}+w_{2} \sigma_{2}^{2} /\left(w_{1}+w_{2}\right)}$, where $w_{1}, w_{2}$ and $\sigma_{1}, \sigma_{2}$ denote the areas and widths of the two Gaussians respectively.
} 

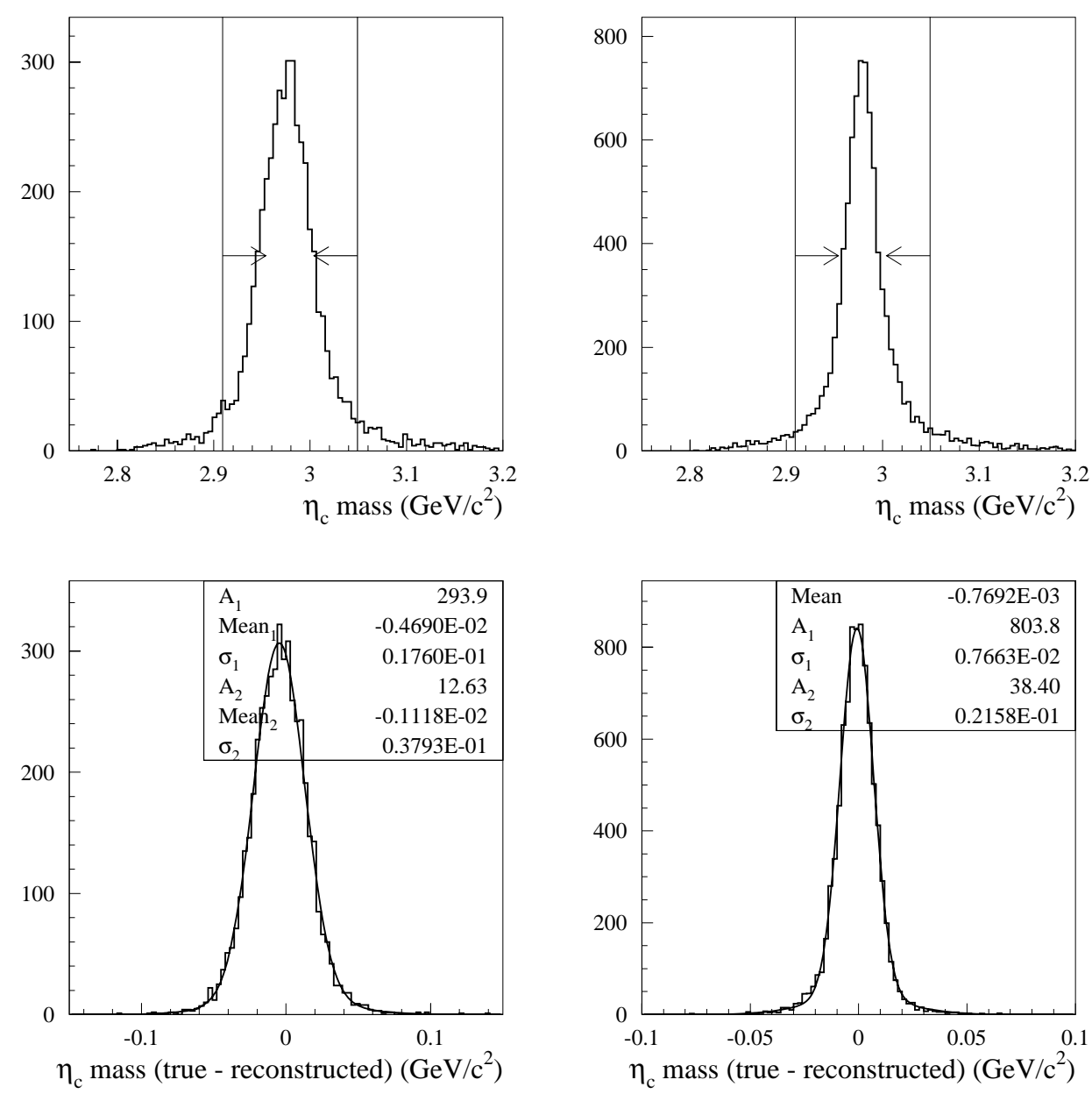

Figure 4.14: Truth-matched $\eta_{c}$ mass (top) and resolution (bottom) Monte Carlo distribution, applying all other selections, for $\eta_{c} \rightarrow K^{+} K^{-} \pi^{0}$ (left) and $\eta_{c} \rightarrow K^{+} K^{-} \pi^{0}$ (right). $B^{+} \rightarrow \eta_{c} K^{+}$Monte Carlo was used. Double Gaussian (common mean, amplitudes $A_{1}, A_{2}$, widths $\sigma_{1}, \sigma_{2}$ ) fits are superimposed on the resolution distributions.

Since the $\eta_{c}$ width is not well known and the Monte Carlo distribution is sensitive to this parameter, selection using the $\eta_{c}$ mass was not optimised. Instead, it was set to the widest selection range used in the Run 1 analysis, $\pm 70 \mathrm{MeV} / c^{2}$ relative to the nominal $\eta_{c}$ mass. 


\subsection{9 $B$ Selection}

The $\Delta E$ and $M_{E S}$ variables are used to select the final $B \rightarrow \eta_{c} K$ candidates. Only the $\Delta E$ selection was optimised, since it was anticipated that the branching fraction measurement would use a fit to the $M_{E S}$ distribution, in which case no $M_{E S}$ selection would be applied ${ }^{4}$.

The $\Delta E$ selection optimisation was performed rather differently to the other selections. $N_{S R}$ was estimated from integrated left-to-right $|\Delta E|$ distribution from signal candidates applying all other selections. $N_{S B}$ was estimated from the integrated left-to-right $|\Delta E|$ distribution from candidates occupying the $M_{E S}$ sideband $\left(M_{E S}<5.269 \mathrm{GeV} / c^{2}\right) . N_{B}$ as a function of the limit on $\Delta E$ was given by

$$
N_{B}=N_{S B} \cdot R_{A},
$$

where in this case, $R_{A}$ was defined as the ratio of the ARGUS integral in the $M_{E S}$ signal region to that in the $M_{E S}$ sideband, and determined from the $M_{E S}$ distribution in the $|\Delta E|>0.1 \mathrm{GeV}$ sidebands.

The $\Delta E$ distributions from the data (from candidates in the $M_{E S}$ sideband) and signal Monte Carlo are shown in Figure 4.1 and Figure 4.2. The optimisation for the $\eta_{c} \rightarrow K^{+} K^{-} \pi^{0}$ channel is illustrated by Figure 4.15 , with the optimal selection found to be $|\Delta E|<0.05 \mathrm{GeV}$ for both $B^{0} \rightarrow \eta_{c} K_{S}^{0}$ and $B^{+} \rightarrow \eta_{c} K^{+}$. For the $B \rightarrow \eta_{c} K, \eta_{c} \rightarrow K_{S}^{0} K^{+} \pi^{-}$channel the optimal $\Delta E$ selection was determined to be $|\Delta E|<0.035 \mathrm{GeV}$.

\footnotetext{
${ }^{4}$ In fact, this was not how the branching fraction was ultimately evaluated.
} 

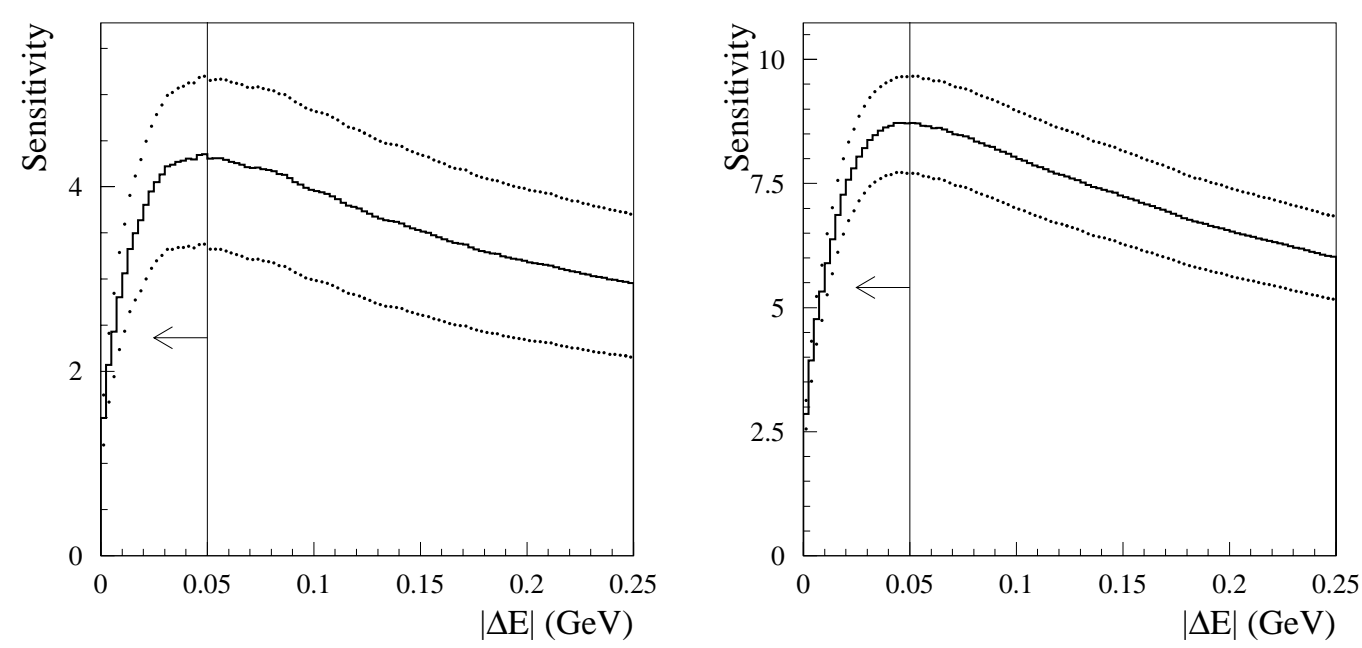

Figure 4.15: $\Delta$ E selection optimisation for $\eta_{c} \rightarrow K^{+} K^{-} \pi^{0}\left(B^{0} \rightarrow \eta_{c} K_{S}^{0}\right.$, left; $B^{+} \rightarrow \eta_{c} K^{+}$, right). Signal and background $\Delta E$ distributions can be seen in Figures 4.1, 4.2. The central (line) and $\pm 1 \sigma$ (dots) sensitivity curves obtained from the optimisation procedure are plotted, with all other selections applied. The arrows denote the optimal selection cuts.

\subsubsection{Resolving Multiple Candidates}

Multiple $B \rightarrow \eta_{c} K$ candidates per event (in a given $\eta_{c} \rightarrow K \bar{K} \pi$ channel) were not permitted in this analysis. To ensure a maximum of one candidate per event, the candidate with the smallest value of $|\Delta E|$ was chosen if multiple candidates survived the selection. This procedure follows that used in other exclusive charmonium analyses at BABAR.

Since the final background estimation in the branching fraction measurement (see Section 5.4) used the full $\Delta E, M_{E S}, m\left(\eta_{c}\right) 3$-dimensional region, the multiple candidates were resolved before applying $\Delta E, M_{E S}, m\left(\eta_{c}\right)$ selections so that all areas of the region were treated consistently. 
Multiple candidates were not resolved in the selection optimisation procedure. This did not affect the $N_{S}$ estimated since truth-matched candidates were used. The number of candidates in the $\Delta E$ sidebands (from which $N_{B}$ was estimated) after all other selections was reduced by approximately $10 \%$ after resolving the multiple candidates.

However, the effect of this overestimation of the background on the sensitivity scale is much less than $10 \%$, depending on the absolute values of $N_{S}$ and $N_{B}$ (assuming that resolving the multiple candidates does not affect the shape of any of the selection variable distributions). For example, for $N_{S} \sim N_{B}$, the fractional change in the sensitivity from a $10 \%$ change in $N_{B}$ is $\sim 3 \%$.

\subsubsection{Fiducial Selections}

In addition to the selections described above, the momenta and angular range of the charged kaon candidates are restricted to regions where the neural network selector's performance is well measured in data and Monte Carlo. Only kaons with polar angle $0.35 \mathrm{rad}<\theta_{\text {kaon }}<2.54 \mathrm{rad}$ and momentum $p_{\text {kaon }}>250 \mathrm{MeV} / c$ are accepted.

\subsubsection{Summary of Optimisation}

The reliability of the sensitivity optimisation depends on the reliability of the $N_{S}$ and $N_{B}$ estimates, which may not be fully accurate for several reasons.

As mentioned above, the optimisation was performed allowing multiple $B \rightarrow \eta_{c} K$ candidates per event, which is not permitted in the final analysis. The procedure used truth-matched signal Monte Carlo candidates and so assumes $100 \%$ truthmatching efficiency. In the estimation of the background, it was assumed that it 
can be described solely by the ARGUS function; this may not be true since there may also be a background component that peaks in $M_{E S}$ at around the $B$ mass (see Section 5.4.2). In extrapolating the background in the $\Delta E, M_{E S}$ signal region from the $\Delta E$ sidebands, the ARGUS shape was assumed to be independent of $\Delta E$.

However, the largest errors in the optimisation are those on the measured branching fractions $B \rightarrow \eta_{c} K$, which were taken into account when plotting the sensitivity. The optimal selection values were not greatly influenced by this error, as can be seen from the sensitivity curves plotted. Since the optimisation is only a guide to maximising the statistical accuracy of the branching fraction, and no measurements are inferred from the optimisation procedure itself, a more detailed analysis taking into account all the effects mentioned above was not carried out.

\subsection{Summary of Selections}

The summary of selections described above is displayed in Table 4.2. As can be seen the optimal selection values obtained for the $\eta_{c} \rightarrow K^{+} K^{-} \pi^{0}$ channels agree very well with the independently obtained values for the $\eta_{c} \rightarrow K_{S}^{0} K^{+} \pi^{-}$channels, for common selection variables (fast $K_{S}^{0}$ variables and the Fisher discriminant).

The final efficiencies for these selections, measured by the number of candidates surviving all the selections divided by the number of generated $B \rightarrow \eta_{c} K$ signal Monte Carlo events are given in Chapter 5, Section 5.6.2. 
Table 4.2: Summary of $B$ candidate selection for $B \rightarrow \eta_{c} K, \eta_{c} \rightarrow K \bar{K} \pi$ and $\eta_{c} \rightarrow$ $K_{S}^{0} K^{+} \pi^{-}$. The selections are almost all uniform between $B^{0} \rightarrow \eta_{c} K_{S}^{0}$ and $B^{+} \rightarrow$ $\eta_{c} K^{+}$channels, where they are different, the selection cut for the $B^{+} \rightarrow \eta_{c} K^{+}$is given in parenthesis.

\begin{tabular}{|c|c|c|}
\hline$\eta_{c}$ Decay Channel & $K_{S}^{0} K^{+} \pi^{-}$ & $K^{+} K^{-} \pi^{0}$ \\
\hline \multicolumn{3}{|l|}{$B$ selection } \\
\hline$|\Delta E|$ & $<35 \mathrm{MeV}$ & $<50 \mathrm{MeV}$ \\
\hline $\mid M_{E S}$ - nominal $B$ mass $\mid$ & $<8 \mathrm{MeV}$ & $<10 \mathrm{MeV}$ \\
\hline$\left|\left(\cos \theta_{H}\right)^{B}\right|$ & $<1.2$ & $<1.2$ \\
\hline Fisher & $>-0.08(>0.0)$ & $>0.0$ \\
\hline \multicolumn{3}{|l|}{$B$ daughter kaon selection } \\
\hline Fast Kaon ID & $\mathrm{VL}$ & VL \\
\hline Fast $\mid K_{S}^{0}$ mass - nominal mass $\mid$ & $<13 \mathrm{MeV} / c^{2}$ & $<13 \mathrm{MeV} / c^{2}$ \\
\hline Fast $K_{S}^{0}$ normalised flight length & $>4$ & $>4$ \\
\hline Fast $\cos \alpha\left(K_{S}^{0}\right)$ & $>0.9995$ & $>0.9995$ \\
\hline \multicolumn{3}{|l|}{$\eta_{c}$ daughter kaon selection } \\
\hline Slow Kaon ID & NAP & $\mathrm{VL}$ \\
\hline Slow $\mid K_{S}^{0}$ mass - nominal mass $\mid$ & $<16 \mathrm{MeV} / c^{2}$ & - \\
\hline Slow $K_{S}^{0}$ normalised flight length & $>4$ & - \\
\hline Slow $\cos \alpha\left(K_{S}^{0}\right)$ & $>0.993$ & - \\
\hline \multicolumn{3}{|l|}{$\pi^{0}$ selection } \\
\hline $\mid \pi^{0}$ mass - nominal mass $\mid$ & - & $<16 \mathrm{MeV} / c^{2}$ \\
\hline $\operatorname{LAT}(\gamma)$ & - & $>0.01$ \\
\hline$E_{\gamma}$ (lower energy) & - & $>40 \mathrm{MeV}$ \\
\hline$E_{\gamma}$ (higher energy) & - & $>230 \mathrm{MeV}$ \\
\hline \multicolumn{3}{|l|}{$\eta_{c}$ selection } \\
\hline $\mid \eta_{c}$ mass - nominal mass $\mid$ & $<70 \mathrm{MeV}$ & $<70 \mathrm{MeV}$ \\
\hline
\end{tabular}




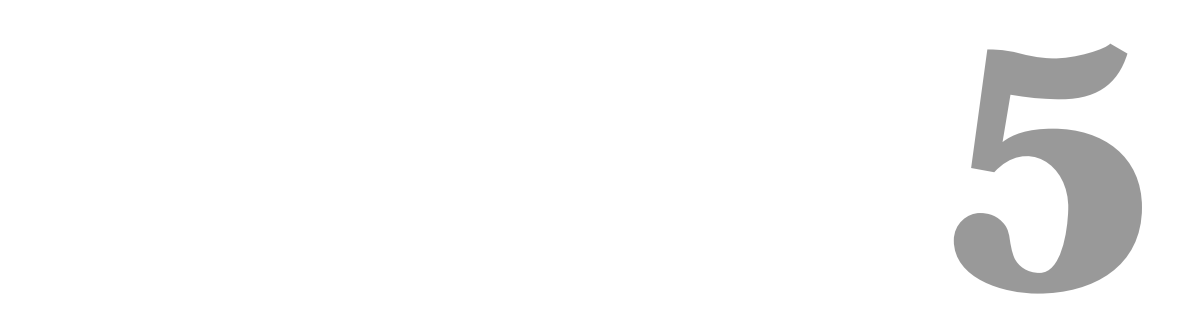

Measurement of Branching

Fraction for $B \rightarrow \eta_{c} K$

\section{$5.1 \quad$ Introduction}

The theoretical motivations for the measurement of the exclusive branching fractions for the decays $B \rightarrow \eta_{c} K$ are outlined in Chapter 1 .

Previous measurements of branching fractions in the $B \rightarrow \eta_{c} K$ channels have been 
performed recently by so-called $B$-Factory experiments (see Chapter 2). These include measurements by the CLEO collaboration using the decay modes $\eta \rightarrow \phi \phi$ and $K_{S}^{0} K^{+} \pi^{-}$[50], and the Belle collaboration using the decay modes $\eta_{c} \rightarrow K_{S}^{0} K^{+} \pi^{-}$, $K^{+} K^{-} \pi^{0}, K^{* 0} K^{-} \pi^{+}$, and $p \bar{p}[23]$.

This analysis measures the branching fractions of $B^{0} \rightarrow \eta_{c} K_{S}^{0}$ and $B^{+} \rightarrow \eta_{c} K^{+}$ where $\eta_{c} \rightarrow K^{+} K^{-} \pi^{0}$ and $\eta_{c} \rightarrow K_{S}^{0} K^{+} \pi^{-}$using the BABAR detector. The first measurement of these branching fractions in BABAR was obtained using the 20.7 $\mathrm{fb}^{-1}$ Run 1 data sample (collected between October 1999 and October 2000) [37] (this was the Run 1 analysis mentioned in Chapter 4). The measurement presented here uses a $79.4 \mathrm{fb}^{-1}$ data sample (collected between October 1999 and July 2002).

The branching fraction for $B \rightarrow \eta_{c} K$ is given by

$$
\mathcal{B}\left(B \rightarrow \eta_{c} K\right)=\frac{N_{Y}-N_{B G}}{N_{B \bar{B}} \epsilon} \frac{1}{\prod_{i} \mathcal{B}_{i}},
$$

where $N_{Y}$ is the total number of $B \rightarrow \eta_{c} K$ candidates selected in the data (the yield), $N_{B G}$ is the estimated number of selected background candidates, $N_{B \bar{B}}$ is the estimated total number of $B$ mesons in the data sample, and $\epsilon$ is the measured selection efficiency. $\mathcal{B}_{i}$ are the branching fractions of secondary decays involved in the decay chain that results in the final state from which the $B$ candidates are reconstructed. For example, when measuring the branching fraction $\mathcal{B}\left(B^{0} \rightarrow \eta_{c} K^{0}\right)$ where $\eta_{c} \rightarrow K \bar{K} \pi$, the branching fractions of $\eta_{c} \rightarrow K \bar{K} \pi$ and $K_{S}^{0} \rightarrow \pi^{+} \pi^{-}$are required, as well as those of the decays of the $\eta_{c}$ daughters (which may include $\pi^{0} \rightarrow \gamma \gamma$ or $\left.K_{S}^{0} \rightarrow \pi^{+} \pi^{-}\right)$.

The calculation of the branching fraction by (5.1) assumes that all the $\eta_{c}$ decay modes which contribute to $\eta_{c} \rightarrow K \bar{K} \pi$ are included correctly in the Monte Carlo. The Run 1 analysis considered only non-resonant $\eta_{c} \rightarrow K \bar{K} \pi$. Since then, evidence has arisen for $\eta_{c} \rightarrow K^{*} \bar{K}$ contributions (see Section 1.6.3). The consideration of the effect of these contributions on the measured branching fraction is a major part of 
this analysis.

This chapter is organised in the following way. The Monte Carlo selection efficiency measurement method is first described, which has been developed to take into account $\eta_{c} \rightarrow K \bar{K} \pi$ decays through intermediate resonances, using a $\eta_{c}$ decay phase space efficiency parametrization. Then methods used to account for differences in Monte Carlo simulation and real event data are discussed, which have been extensively developed at $B A B A R$. The estimation of the background contributions in $B \rightarrow \eta_{c} K$ reconstruction is then described. Finally the branching fraction results on the full $79.4 \mathrm{fb}^{-1}$ sample are given.

\subsection{Monte Carlo Efficiency Measurement}

The selection efficiency $\epsilon$ is measured using the signal $B \rightarrow \eta_{c} K, \eta_{c} \rightarrow K \bar{K} \pi \mathrm{SP} 4$ Monte Carlo samples described in Section 4.2.2. A simple measurement of the efficiency is given by the number of reconstructed candidates surviving all selections divided by the number of generated events.

However since only the non-resonant $\eta_{c} \rightarrow K \bar{K} \pi$ decays are simulated in the signal Monte Carlo, a measurement of the efficiency as described above may not describe the true efficiency. The real event data may contain $\eta_{c} \rightarrow K^{*} \bar{K} \rightarrow K \bar{K} \pi$ events, and variations of efficiency in the $\eta_{c}$ decay phase space may lead to different efficiencies for non-resonant $\eta_{c} \rightarrow K \bar{K} \pi$ and $\eta_{c} \rightarrow K^{*} \bar{K} \rightarrow K \bar{K} \pi$ reconstruction and selection. To take the phase space efficiency variation into account in the branching fraction calculation, a differential efficiency correction over the $\eta_{c} \rightarrow K \bar{K} \pi$ Dalitz plot is required. The same kind of situation has been encountered in other BABAR analyses examining three-body decays, such as $B \rightarrow h h h$ [51] and $D^{0} \rightarrow h h h[52]$ (where $h$ denotes a kaon or pion). In these analyses the Dalitz plot describing the three-body 
phase space was divided into many bins and the efficiency evaluated for each bin. The branching fraction is then given by, for example

$$
\mathcal{B}(B \rightarrow h h h)=\frac{1}{N_{B \bar{B}}} \sum_{i}^{N_{\text {bins }}} \frac{N_{Y}^{i}-N_{B}^{i}}{\epsilon^{i}},
$$

where $N_{Y}^{i}, N_{B}^{i}, \epsilon^{i}$ are the total number of events, number of background events, and efficiency respectively in bin $i$ of the $B \rightarrow h h h$ Dalitz plot.

\subsubsection{Efficiency in the $\eta_{c} \rightarrow K \bar{K} \pi$ Dalitz Plot}

The conventional Dalitz plots for reconstructed $\eta_{c} \rightarrow K^{+} K^{-} \pi^{0}$ and $\eta_{c} \rightarrow K_{S}^{0} K^{+} \pi^{-}$ events (using SP4 $B \rightarrow \eta_{c} K$ Monte Carlo), applying the full selection described in Chapter 4 are shown in Figure 5.1. For $\eta_{c} \rightarrow K_{S}^{0} K^{+} \pi^{-}$the scatter plot of $m_{K_{S}^{0} \pi^{-}}^{2}$ vs. $m_{K^{+} \pi^{-}}^{2}$ is drawn, while for $\eta_{c} \rightarrow K^{+} K^{-} \pi^{0}$ the scatter plot of $m_{K^{+} \pi^{0}}^{2}$ vs. $m_{K^{-} \pi^{0}}^{2}$ (charge conjugates not implied) is shown.
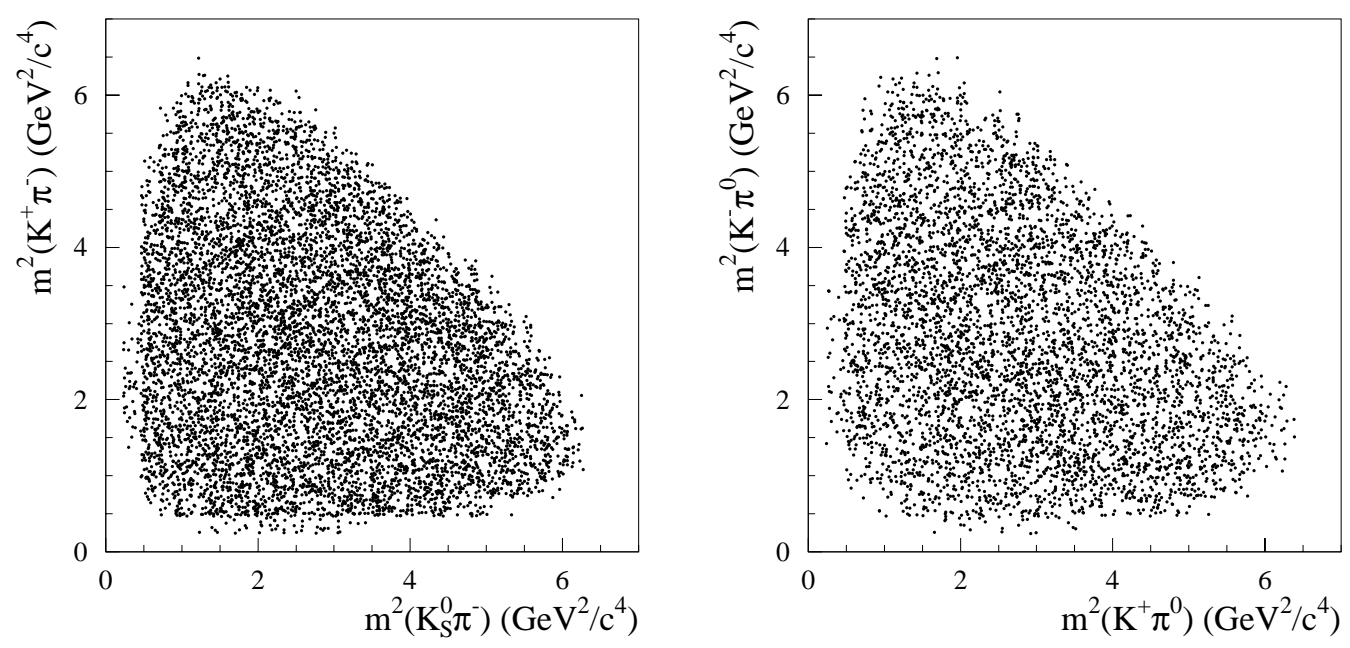

Figure 5.1: Dalitz plots for reconstructed $B^{0} \rightarrow \eta_{c} K_{S}^{0}$ Monte Carlo where $\eta_{c} \rightarrow K_{S}^{0} K^{+} \pi^{-}$(left) and $\eta_{c} \rightarrow K^{+} K^{-} \pi^{0}$ (right). In the $\eta_{c} \rightarrow K^{+} K^{-} \pi^{0}$ Dalitz plot, charge conjugates are not implied in the two variables. 
The plots show the expected uniform distribution for non-resonant $\eta_{c} \rightarrow K \bar{K} \pi$ simulation. The efficiency distributions in the two projections of each Dalitz plot are obtained by dividing the distributions in Figure 5.1 by those obtained from the generated Monte Carlo events. The efficiency distributions are shown in Figure 5.2. These show some degree of efficiency dependence on $m_{K \pi}$ and $m_{\bar{K}_{\pi}}$. The variation of the efficiency probably arises from a number of reconstruction and selection effects (such as particle identification and photon selection requirements) which restrict the momentum spectra of the $\eta_{c}$ daughters, thereby restricting the occupancy of the invariant mass distributions in certain regions.
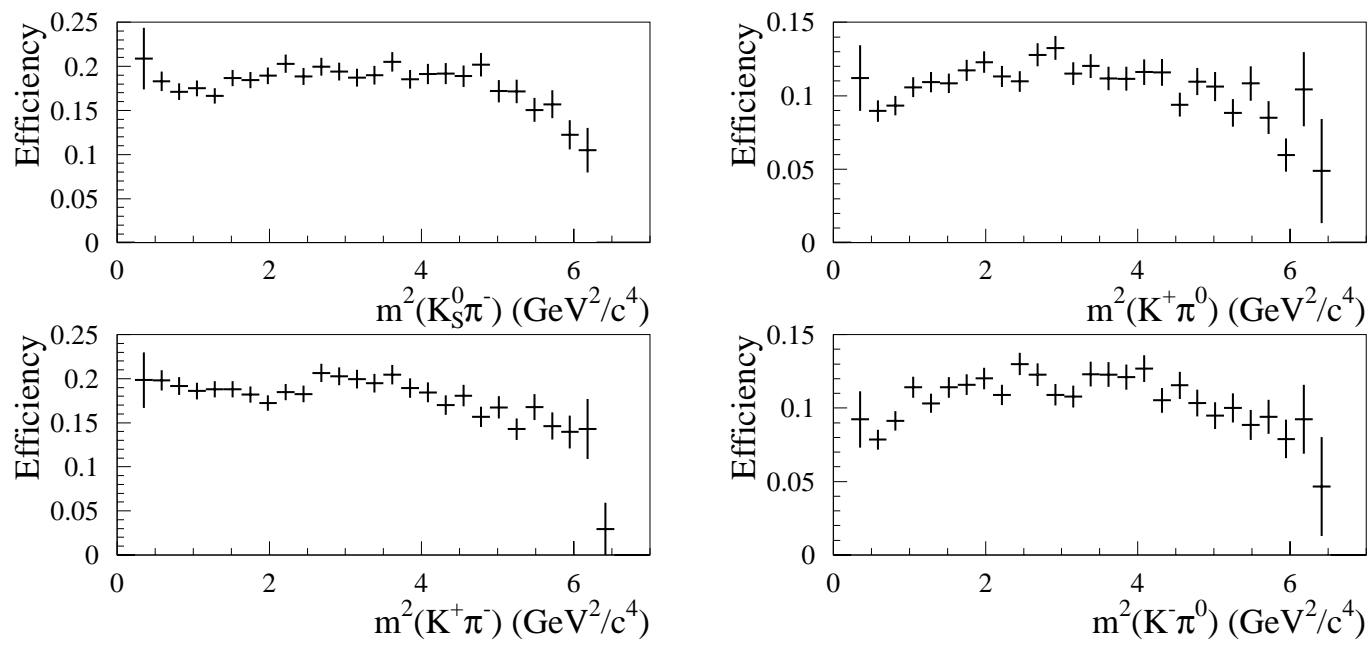

Figure 5.2: Monte Carlo efficiency vs. Dalitz plots variables for $\eta_{c} \rightarrow$ $K_{S}^{0} K^{+} \pi^{-}$(left) and $\eta_{c} \rightarrow K^{+} K^{-} \pi^{0}$ (right). $B^{0} \rightarrow \eta_{c} K_{S}^{0}$ Monte Carlo was used.

As mentioned before, the SP4 signal $B \rightarrow \eta_{c} K$ Monte Carlo contains only nonresonant $\eta_{c} \rightarrow K \bar{K} \pi$ decays. To study the structure of, and efficiency for, $\eta_{c} \rightarrow$ $K^{*}(1430) \bar{K} \rightarrow K \bar{K} \pi$ decays in the Dalitz plot, a fast Monte Carlo production tool was used, called PravdaMC (see Appendix E).

The structure of $\eta_{c} \rightarrow K^{*}(1430) \bar{K} \rightarrow K \bar{K} \pi$ decays in the Dalitz plots, using PravdaMC Monte Carlo is shown in Figure 5.3. Resonance bands are clearly visible at $m_{K_{S}^{0} \pi^{-}}$, 
$m_{K \pi} \approx 1430 \mathrm{GeV} / c^{2}$. In each plot, the invariant mass squared of one of the $K^{*}$ daughters and the bachelor kaon is proportional to the helicity cosine of the $K^{*}$ (see Appendix C). Thus for $\eta_{c} \rightarrow K_{2}^{*}(1430) \bar{K}$ the helicity cosine of the $K_{2}^{*}$ follows the square of a second order Legendre polynomial and this explains the intensity variation as a function of $m_{K \pi}$ in the $K_{2}^{*}$ bands. Similarly for $\eta_{c} \rightarrow K_{0}^{*}(1430) \bar{K}$, the helicity cosine of the $K_{0}^{*}$ is uniform and so the intensity as a function of $m_{K \pi}$ is uniform in the $K_{0}^{*}$ bands.
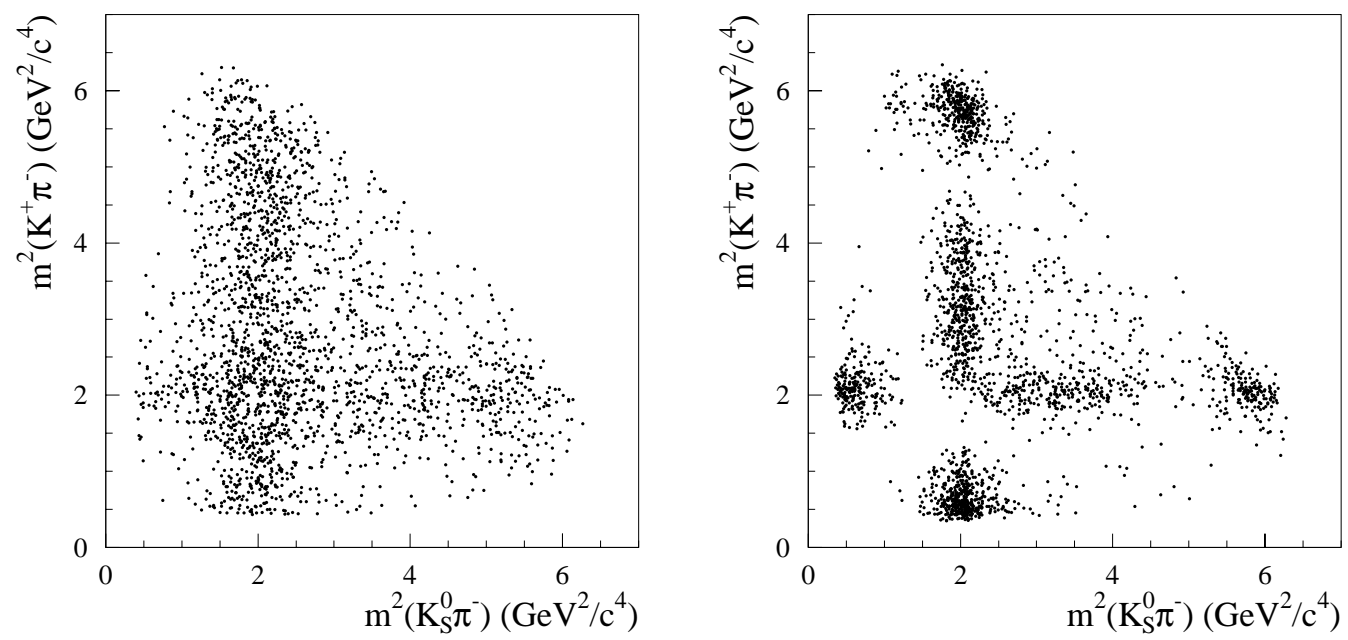

Figure 5.3: Dalitz plots for reconstructed $B^{0} \rightarrow \eta_{c} K_{S}^{0}$ MC where $\eta_{c} \rightarrow$ $K_{0}^{*}(1430) \bar{K} \rightarrow K_{S}^{0} K^{+} \pi^{-}$(left) and $\eta_{c} \rightarrow K_{2}^{*}(1430) \bar{K} \rightarrow K_{S}^{0} K^{+} \pi^{-}$(right).

An efficiency study was carried out on simulated $B \rightarrow \eta_{c} K, \eta_{c} \rightarrow K_{S}^{0} K^{+} \pi^{-}$events using PravdaMC including non-resonant $\eta_{c} \rightarrow K_{S}^{0} K^{+} \pi^{-}$decays, and those through the intermediate states $K_{0}^{*}(1430) \bar{K}, K_{2}^{*}(1430) \bar{K}$. All the selections described in Chapter 4 were applied; the results are shown in Table 5.1.

The efficiency for each type of $\eta_{c}$ decay in Table 5.1 is almost identical. However, the efficiency of reconstruction in PravdaMC does not reliably reproduce that in SP4 (or hence, in data); the Dalitz plot efficiency projections for $\eta_{c} \rightarrow K_{S}^{0} K^{+} \pi^{-}$ (non-resonant) using PravdaMC can be seen in Figure 5.4. Apart from the obvious 
Table 5.1: Measured selection efficiency using PravdaMC simulated $B^{0} \rightarrow \eta_{c} K_{S}^{0}$ events where $\eta_{c} \rightarrow K_{S}^{0} K^{+} \pi^{-}$(non-resonant), $\eta_{c} \rightarrow K_{0}^{*}(1430) \bar{K} \rightarrow K_{S}^{0} K^{+} \pi^{-}, \eta_{c} \rightarrow$ $K_{2}^{*}(1430) \bar{K} \rightarrow K_{S}^{0} K^{+} \pi^{-}$. 6,000 events of each $\eta_{c}$ decay type were generated.

\begin{tabular}{c|c|c|c}
\hline$\eta_{c}$ Decay Channel & $K_{S}^{0} K^{+} \pi^{-}$ & $K_{0}^{*}(1430) \bar{K}$ & $K_{2}^{*}(1430) \bar{K}$ \\
\hline Efficiency (\%) & $28.1 \pm 0.6$ & $29.2 \pm 0.6$ & $28.2 \pm 0.6$ \\
\hline
\end{tabular}
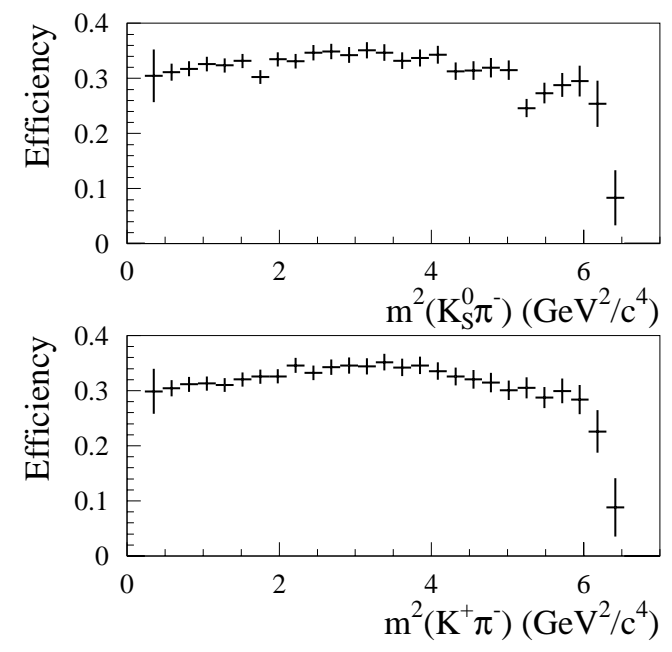

Figure 5.4: PravdaMC Monte Carlo efficiency projections of Dalitz plots for $B^{0} \rightarrow \eta_{c} K_{S}^{0}, \eta_{c} \rightarrow K_{S}^{0} K^{+} \pi^{-}$.

difference in absolute efficiency between SP4 and PravdaMC, the efficiency variation in PravdaMC is visibly different to that in SP4. Thus it is not safe to assume a uniform efficiency across the resonant and non-resonant $\eta_{c} \rightarrow K_{S}^{0} K^{+} \pi^{-}$decay modes, in which case a single efficiency value could be used in the $\mathcal{B}\left(B \rightarrow \eta_{c} K\right)$ measurement rather than employing a differential efficiency correction.

A similar efficiency study for the $\eta_{c} \rightarrow K^{+} K^{-} \pi^{0}$ channel was not carried out, since the photon reconstruction simulation in PravdaMC is particularly naive, resulting in a very poor $\pi^{0}$ simulation. 
In conclusion, the efficiency variations observed in the $\eta_{c} \rightarrow K \bar{K} \pi$ phase space in SP4 Monte Carlo must be accounted for in the $\mathcal{B}\left(B \rightarrow \eta_{c} K\right)$ measurement, as in other three-body analyses, by a differential efficiency correction, which is discussed in the next sections.

\subsubsection{An Alternative Representation of the Dalitz Plot}

Using the conventional Dalitz plot to provide a differential efficiency correction over the three-body phase space (as in (5.2)) has practical disadvantages. Measuring the Monte Carlo efficiency at the boundary of the kinematically allowed region of the Dalitz plot (with finite Monte Carlo statistics) is difficult, since the boundary is highly non-rectilinear. The efficiency measurement in Dalitz plot bins at or near the position of the boundary will suffer from low occupancy and poor statistical precision.

These problems may be somewhat alleviated by representing the phase space in variables that have orthogonal boundaries [53]. Three-body phase space may be represented by the helicity cosine and the invariant mass of a given two-body subsystem, as mentioned in Section 1.6.3. This pair of variables has orthogonal kinematic boundaries. For the $\eta_{c} \rightarrow K \bar{K} \pi$ decay, the transformation of the conventional Dalitz plot variables to those described above is demonstrated in Appendix C.

For the $\eta_{c} \rightarrow K_{S}^{0} K^{+} \pi^{-}$decay the phase space population can be described by, for example

$$
d N \propto q p_{\pi^{-}} d m_{K_{S}^{0} \pi^{-}} d\left(\cos \theta_{H}\right)^{K_{S}^{0} \pi^{-}},
$$

where $q$ is the $K^{+}$momentum in the $\eta_{c}$ rest frame, and $p_{\pi^{-}}$is the $\pi^{-}$momentum in the $K_{S}^{0} \pi^{-}$rest frame (these momenta can be calculated from two-body energy-momentum conservation formulae as shown in Appendix C). The plot of $\left(\cos \theta_{H}\right)^{K_{S}^{0} \pi^{-}}$vs. $m_{K_{S}^{0} \pi^{-}}$using Monte Carlo generated quantities is shown in Figure 
5.5. The shapes of the distributions in the $\left(\cos \theta_{H}\right)^{K_{S}^{0} \pi^{-}}$and $m_{K_{S}^{0} \pi^{-}}$projections correspond to those expected from the theoretical phase space population (5.3).

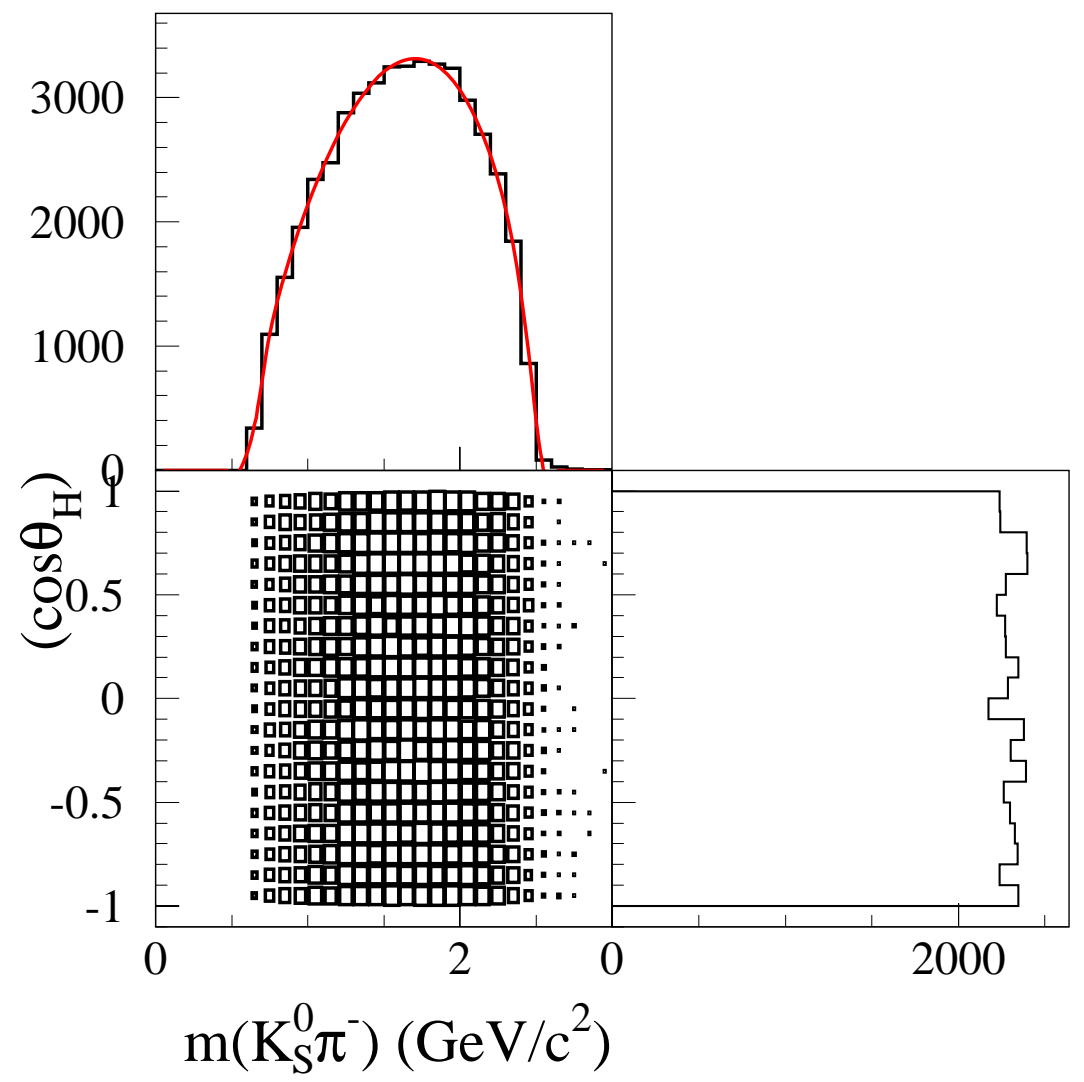

Figure 5.5: Scatter plot, with projections, of $m_{K_{S}^{0} \pi^{-}}$(horizontal axis) vs. corresponding helicity cosine $\left(\cos \theta_{H}\right)^{K_{S}^{0} \pi^{-}}$(vertical axis) for generated $\eta_{c} \rightarrow$ $K_{S}^{0} K^{+} \pi^{-}$Monte Carlo (from $B^{+} \rightarrow \eta_{c} K^{+}$decays). A fit to the $m_{K_{S}^{0} \pi^{-}}$projection, using the predicted shape from (5.3) is superimposed.

Any two-body subsystem from the $K \bar{K} \pi$ system may be chosen and the helicity cosine plotted against the invariant mass to illustrate the phase space. A method to determine the efficiency as a function of the phase space variables is described in the next section. 


\subsubsection{Efficiency Parametrization}

The alternative representation of the $\eta_{c}$ decay phase space allows a semi-continuous parametrization of the phase space efficiency, which can be constructed as follows.

The generated and reconstructed helicity cosine distributions can be parametrized by Legendre polynomials $P_{l}(\cos \theta)$. The generated helicity distribution, which is uniform, is described by

$$
\frac{d N_{0}}{d \cos \theta}=N_{0} P_{0}(\cos \theta),
$$

where $N_{0}$ is the total number of generated candidates. The reconstructed distribution can be parametrized as a linear combination of Legendre polynomials $P_{i}(\cos \theta)$, with coefficients $c_{i}$

$$
\frac{d N_{\text {reco }}}{d \cos \theta}=N_{\text {reco }} \sum_{i} c_{i} P_{i}(\cos \theta),
$$

where $N_{\text {reco }}$ is the number of reconstructed candidates. The Legendre polynomials

are normalised such that $\int_{-1}^{+1} P_{m} P_{n} d \cos \theta=\delta_{m n}$. Using the orthogonality of the Legendre polynomials, the coefficients $c_{i}$ are obtained by

$$
c_{i}=\frac{1}{N_{\text {reco }}} \int_{-1}^{+1} \frac{d N_{\text {reco }}}{d \cos \theta} P_{i}(\cos \theta) d \cos \theta,
$$

which can be approximated, for a large enough sample, by

$$
c_{i}=\frac{1}{N_{\text {reco }}} \sum_{j=1}^{N_{\text {reco }}} P_{i}\left(\cos \theta_{j}\right)=<P_{i}(\cos \theta)>,
$$

which is just the mean value of the $P_{i}$ polynomial; the error on $c_{i}$ is then given by the standard error on $\left\langle P_{i}\right\rangle$.

The efficiency as a function of $\cos \theta$ is then given by

$$
\epsilon(\cos \theta)=\frac{d N_{\text {reco }}}{d \cos \theta} / \frac{d N_{0}}{d \cos \theta}=\frac{N_{\text {reсo }} \sum_{i} c_{i} P_{i}(\cos \theta)}{N_{0} P_{0}(\cos \theta)} .
$$

To extend the efficiency parametrization to cover the two-dimensional phase space, the parametrization in the two-body helicity cosine described above must be re- 
peated in several intervals of the corresponding two-body invariant mass. The efficiency for a candidate with mass, helicity $\operatorname{cosine}$ values $m, \cos \theta$, where $m$ lies in the interval labelled by $j$, is given by

$$
\epsilon(m, \cos \theta)=\frac{d N_{r e c o}^{j}}{d \cos \theta} / \frac{d N_{0}^{j}}{d \cos \theta}=\frac{N_{\text {reco }}^{j} \sum_{i} c_{i}^{j} P_{i}(\cos \theta)}{N_{0}^{j} P_{0}(\cos \theta)} .
$$

This prescription for the parametrization the efficiency variation was applied to SP4 signal $B \rightarrow \eta_{c} K$ Monte Carlo. Two parametrizations of the phase space efficiency were made for each of the $\eta_{c} \rightarrow K \bar{K} \pi$ channels, those involving the two-body systems containing the pion. The highest order of Legendre polynomial and number of invariant mass intervals necessary to model the efficiency was determined empirically. Figures 5.6 and 5.7 illustrate the four efficiency parametrizations in $B^{0} \rightarrow \eta_{c} K_{S}^{0}$ Monte Carlo, where 10 mass slices were used over the kinematically allowed range. Since the upper limit on the two-body invariant mass is governed by the $\eta_{c}$ mass, which is not a fixed value (see Section 5.2.5), a tail is seen at the upper edge of the two-body invariant mass distribution. The mass slices were therefore chosen to cover the range, for the $m_{K_{A} \pi}$ mass,

$$
m_{K_{A}}+m_{\pi}<m_{K_{A} \pi}<m\left(\eta_{c}\right)+4 \Gamma\left(\eta_{c}\right)-m_{K_{B}},
$$

where $\Gamma\left(\eta_{c}\right)=27 \mathrm{MeV} / c^{2}$, the generated $\eta_{c}$ width in the Monte Carlo. Using this range approximately $0.1 \%$ of the generated events lie above the upper limit in $m_{K_{A} \pi}$.

It can be seen that including Legendre polynomials up to fourth order is not necessary to describe the efficiency variation, since most of the $c_{3}, c_{4}$ coefficients in the various mass slices are consistent with zero. However, it is a common feature that the efficiency tends to fall where the helicity cosine is near \pm 1 , particularly for the $K^{+} \pi^{0}$ and $K^{-} \pi^{0}$ systems in $\eta_{c} \rightarrow K^{+} K^{-} \pi^{0}$; this is indicated by the negative values of the $c_{2}$ coefficients in many mass slices. 


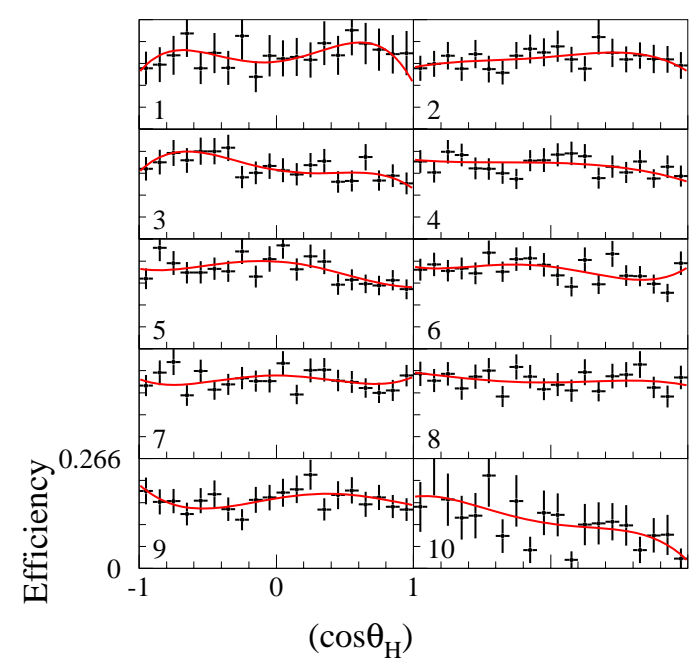

(a)
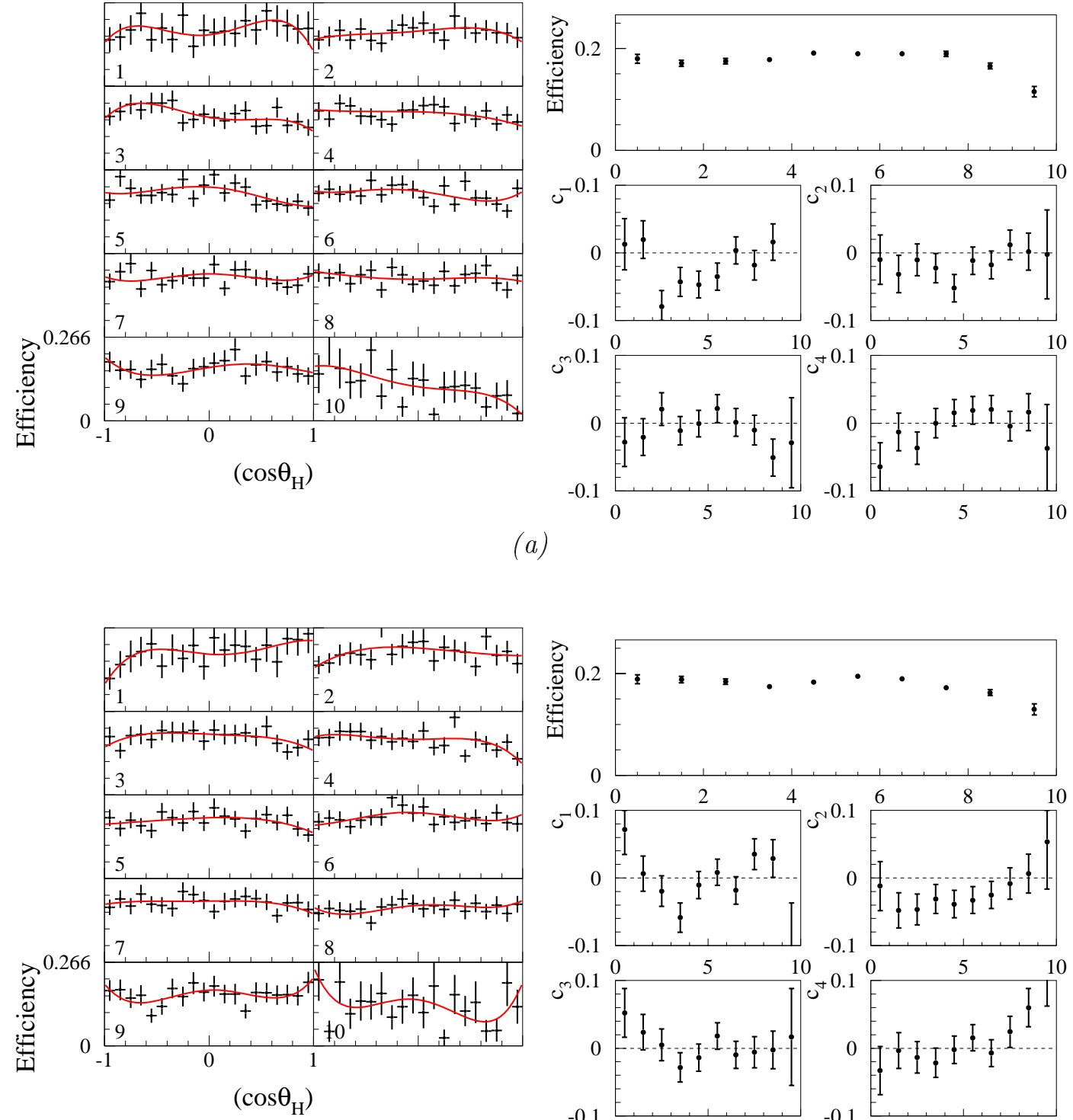

(b)

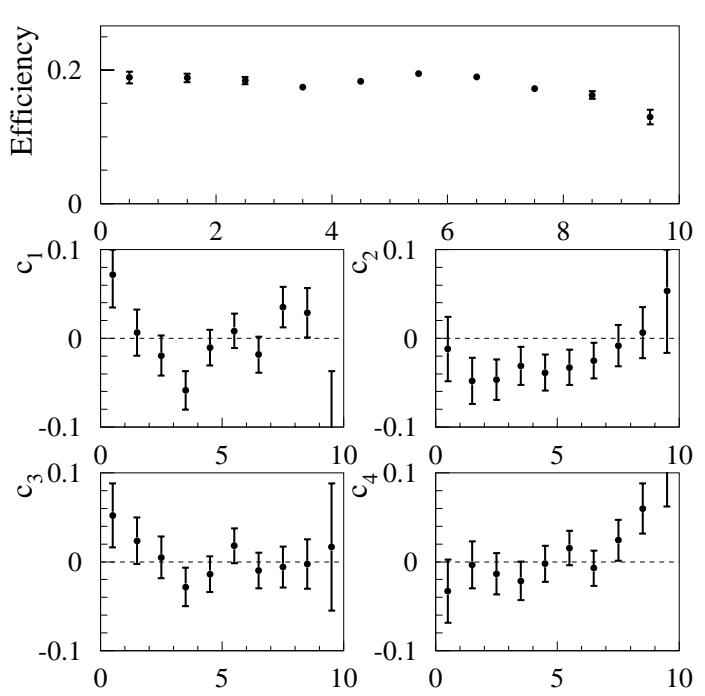

Figure 5.6: Efficiency parametrization for $\eta_{c} \rightarrow K_{S}^{0} K^{+} \pi^{-}$using (a) the $K_{S}^{0} \pi$

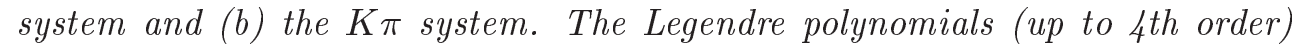
are superimposed on the efficiency vs. helicity cosine plots for each invariant mass slice 1-10 (left). The efficiency and Legendre polynomial coefficients $c_{i}$ for each slice are also plotted (right). 

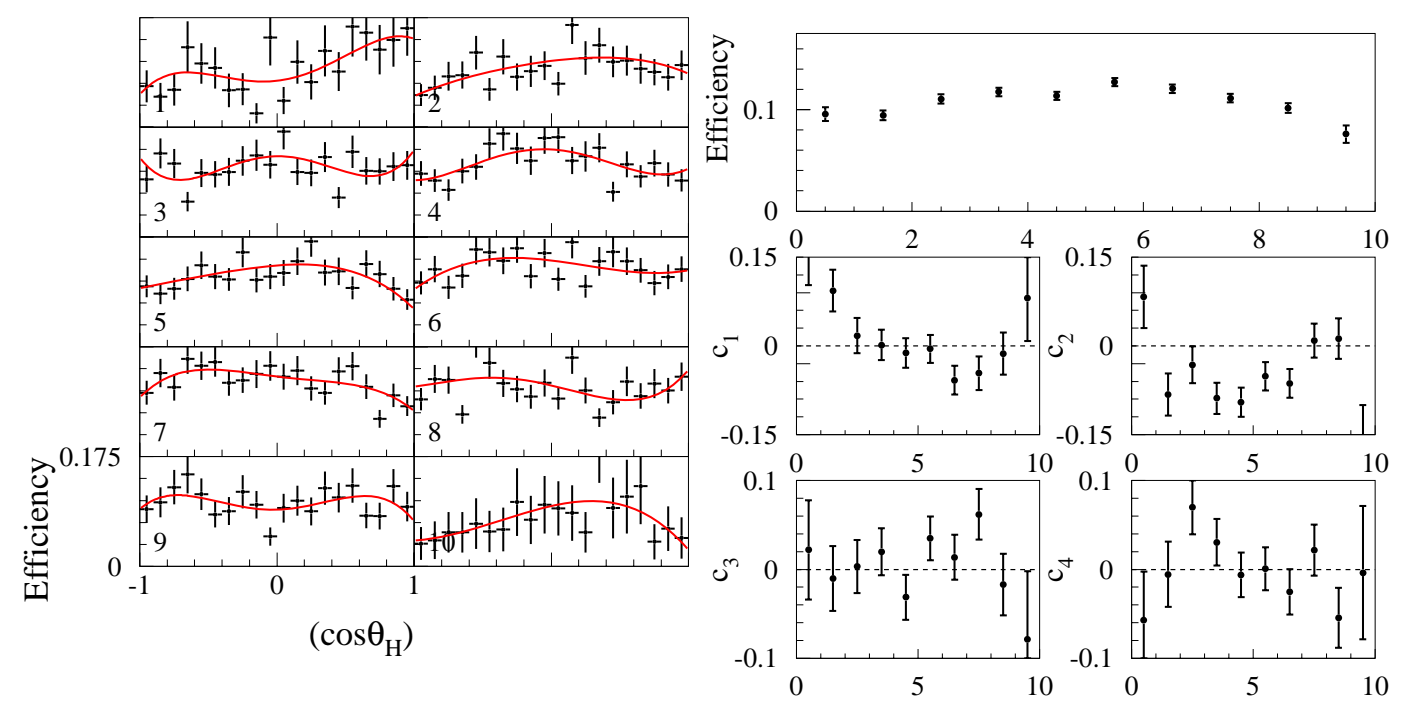

(a)
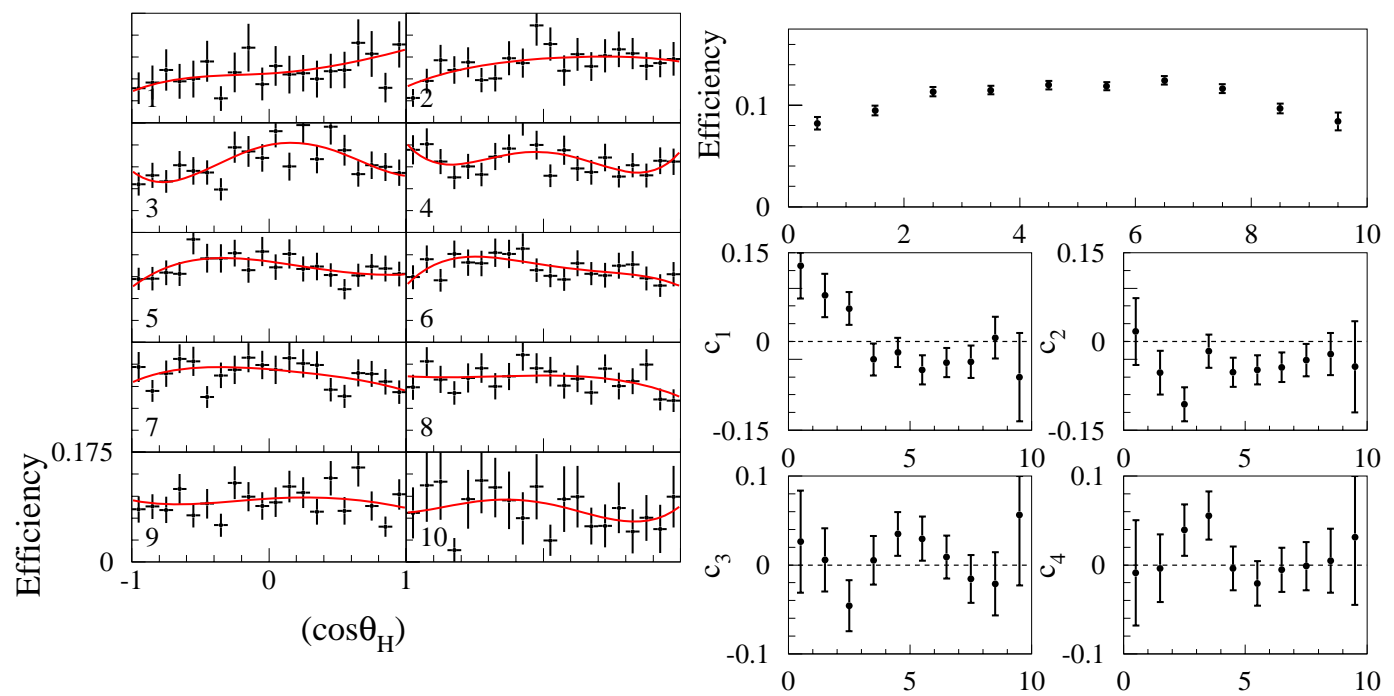

(b)

Figure 5.7: Efficiency parametrization for $\eta_{c} \rightarrow K^{+} K^{-} \pi^{0}$ using (a) the $K^{+} \pi^{0}$ system and (b) the $K^{-} \pi^{0}$ system. See Figure 5.6 for description.

\subsubsection{Efficiency Parametrization Validation}

With the efficiency parametrization in place, each reconstructed candidate can thus be given a weight, depending on its position in the $m, \cos \theta$ plane. The efficiency- 
corrected yield $\mathcal{N}_{Y}$ of sample of events to which the full selection is applied (where $N$ candidates survive) can thus be calculated by the sum-of-weights

$$
\mathcal{N}_{Y}=\sum_{i}^{N} 1 / \epsilon\left(m_{i}, \cos \theta_{i}\right),
$$

and the statistical error can be estimated by

$$
\sigma\left(\mathcal{N}_{Y}\right)=\sqrt{\sum_{i}^{N} 1 / \epsilon^{2}\left(m_{i}, \cos \theta_{i}\right)},
$$

which can be seen to be seen to reduce to $\sqrt{N} / \epsilon$ for the case of a constant efficiency across the phase space.

The efficiency-corrected yield $\mathcal{N}_{Y}$ for signal Monte Carlo candidates after all selections should recover the generated number of events in each $B \rightarrow \eta_{c} K$ channel. This was tested using $\eta_{c} \rightarrow K_{S}^{0} K^{+} \pi^{-}$Monte Carlo, combining the $B^{0}$ and $B^{+}$samples (88,000 generated events) and using the $K \pi$ two-body subsystem parametrization. The relative efficiency variation in the $\eta_{c} \rightarrow K_{S}^{0} K^{+} \pi^{-}$decay phase space should be the same in $B \rightarrow \eta_{c} K$ and $B^{0} \rightarrow \eta_{c} K_{S}^{0}$, although there may be differences in the absolute efficiencies for the $B^{0}$ and $B^{+}$samples. These differences were taken into account when calculating the sum-of-weights. The sample was divided into 440 subsamples, each containing 200 generated events, and the efficiency-corrected yield $\mathcal{N}_{Y}$ computed for each subsample (using Legendre polynomials up to second order) according to (5.11). The resulting distribution of $\mathcal{N}_{Y}^{i}$ values should be binomial, centred at 200. Equivalently if the variable

$$
D_{i}=\frac{\mathcal{N}_{Y}^{i}-200}{\sigma\left(\mathcal{N}_{Y}^{i}\right) \sqrt{1-\epsilon}},
$$

is defined, where $\sigma\left(\mathcal{N}_{Y}\right)$ is calculated by the sum-of-weights (5.12), and $\epsilon$ is the average efficiency for the $B^{0}$ or $B^{+}$sample, then the distribution of $D_{i}$ values should be centred at zero with a standard deviation equal to one.

Following the procedure described above gave $\langle D>=0.072 \pm 0.047$ (consistent 
with zero), with standard deviation $0.979 \pm 0.033$ (consistent with one), thus supporting the method for calculating the efficiency corrected yield and error.

The efficiency parametrization computed using non-resonant $\eta_{c} \rightarrow K \bar{K} \pi(B \rightarrow$ $\left.\eta_{c} K\right)$ Monte Carlo, used to calculate the efficiency-corrected yield for $\eta_{c} \rightarrow K^{*} \bar{K} \rightarrow$ $K \bar{K} \pi\left(B \rightarrow \eta_{c} K\right)$ Monte Carlo candidates should also recover the generated number of events. This was tested using the PravdaMC samples described in Section 5.2.1, applying an additional selection to introduce more efficiency variation in the $\eta_{c} \rightarrow$ $K_{S}^{0} K^{+} \pi^{-}$decay phase space (the momentum of the daughter pion was restricted to $\left.0.3 \mathrm{GeV} / c^{2}<p_{\pi}<2.0 \mathrm{GeV} / c^{2}\right)$. The efficiency variation in the Dalitz plot with this extra requirement is illustrated by Figure 5.8. The efficiency variation seen in SP4 Monte Carlo is not exactly reproduced by this selection, but is greater than that seen in Figure 5.4, and allows a more realistic, non-trivial test of the corrected yield calculation using the sum-of-weights method.

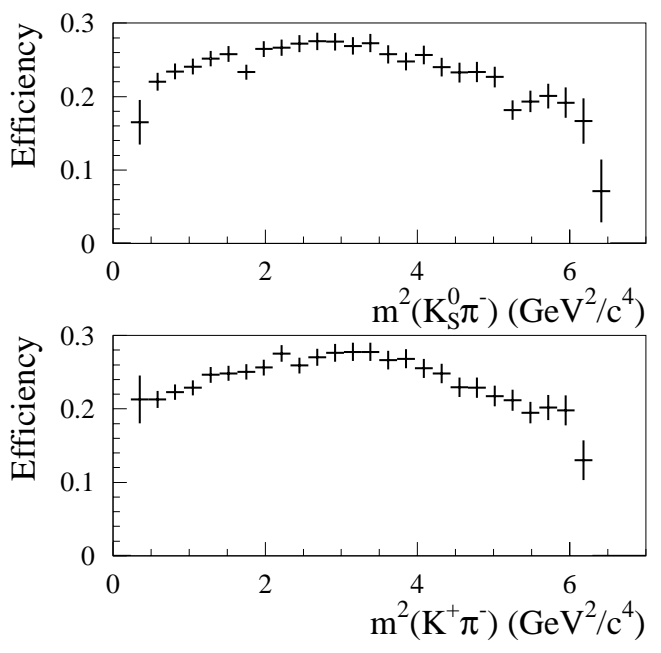

Figure 5.8: PravdaMC Monte Carlo efficiency projections of Dalitz plots for $B^{0} \rightarrow \eta_{c} K_{S}^{0}, \eta_{c} \rightarrow K_{S}^{0} K^{+} \pi^{-}$, with additional selection on the slow pion momentum (see text).

The efficiency-corrected yields were then computed for $\eta_{c} \rightarrow K_{0}^{*}(1430) \bar{K} \rightarrow K_{S}^{0} K^{+} \pi^{-}$ 
and $\eta_{c} \rightarrow K_{2}^{*}(1430) \bar{K} \rightarrow K_{S}^{0} K^{+} \pi^{-}$, and are given in Table 5.2. The yields were calculated using two efficiency parametrizations, based on the $K \pi$ subsystem and the $K_{S}^{0} \pi$ subsystem. In all cases the efficiency-corrected yield was consistent with the generated number of events.

Table 5.2: Efficiency-corrected yields calculated for $B^{0} \rightarrow \eta_{c} K_{S}^{0}, \eta_{c} \rightarrow K^{*} \bar{K} \rightarrow$ $K_{S}^{0} K^{+} \pi^{-}$Monte Carlo (the samples are described in Table 5.1), using efficiency parametrizations computed with $B^{0} \rightarrow \eta_{c} K_{S}^{0}, \eta_{c} \rightarrow K_{S}^{0} K^{+} \pi^{-}$(non-resonant) Monte Carlo.

\begin{tabular}{c|c|c}
\hline$\eta_{c}$ Decay Channel & $K_{0}^{*}(1430) \bar{K}$ & $K_{2}^{*}(1430) \bar{K}$ \\
\hline $\mathcal{N}_{Y}(K \pi$ weights $)$ & $6125.7 \pm 159.4$ & $5935.5 \pm 160.7$ \\
$\mathcal{N}_{Y}\left(K_{S}^{0} \pi\right.$ weights $)$ & $6051.3 \pm 156.3$ & $6153.3 \pm 168.1$ \\
\hline
\end{tabular}

When employing the sum-of-weights method in a full branching fraction measurement on data, signal events obviously cannot be isolated, and the method must be integrated into the background estimation. This is discussed further in Section 5.4.4.

\subsubsection{Sensitivity of the Efficiency Parametrization}

The ideal situation in which to construct an efficiency parametrization as described above would be one in which the the mass of the $\eta_{c}$ is fixed. In that case, the generated Dalitz plot has well defined kinematic boundaries in $m_{K \pi}$ and $m_{\bar{K} \pi}$ (in the reconstructed Dalitz plot, events may fall above the upper kinematic boundaries due to experimental resolution).

In reality, the $\eta_{c}$ mass is not fixed but is described by the $\eta_{c}$ lineshape - a BreitWigner distribution with an appreciable width. The Monte Carlo Dalitz plots shown 
in the previous sections can be thought of as the integrals of many fixed- $\eta_{c}$-mass Dalitz plots over the $\eta_{c}$ mass lineshape. Thus the upper limit (See (C.9), Appendix C) on $m_{K \pi}$ and $m_{\bar{K} \pi}$ is not fixed but is described by the tail of the $\eta_{c}$ mass distribution (just visible in Figure 5.5). In principle, the selection efficiency for a $B \rightarrow \eta_{c} K$, $\eta_{c} \rightarrow K_{A} K_{B} \pi$ event at a fixed position in $m_{K_{A} \pi}, m_{K_{B} \pi}$ may depend on the value of the $\eta_{c}$ mass in that event.

The sensitivity of the efficiency variation on the value of the $\eta_{c}$ mass can be studied in Monte Carlo, fixing the generated $\eta_{c}$ mass to different values and observing the resulting efficiency variation in the Dalitz plot projections.

Using PravdaMc, two $B^{0} \rightarrow \eta_{c} K_{S}^{0}, \eta_{c} \rightarrow K_{S}^{0} K^{+} \pi^{-}$samples were generated where the $\eta_{c}$ mass was fixed to $2.90 \mathrm{GeV} / c^{2}$ and $3.05 \mathrm{GeV} / c^{2}$. The efficiency variation in $m_{K_{S}^{0} \pi^{-}}, m_{K \pi}$ was then compared, applying all selections including the restriction on the slow pion momentum described in Section 5.2.4 (in addition, the $\eta_{c}$ mass selection was modified to $\left.2.90 \mathrm{GeV} / c^{2}<m\left(K_{S}^{0} K^{+} \pi^{-}\right)<3.05 \mathrm{GeV} / c^{2}\right)$. The efficiency comparisons can be seen in Figure 5.9. Since the $\eta_{c}$ mass is generated at the edges of the acceptance range, the average efficiency is about half that in Figure 5.8 (due to experimental resolution). Only the difference in efficiency variation between the two cases shown in Figure 5.9 is important. Apart from the different ranges of $m_{K_{S}^{0} \pi^{-}}, m_{K \pi}$ occupied in the two cases, the efficiency variation does not appear to be systematically different.

Thus the sensitivity of the efficiency parametrization to the generated value of the $\eta_{c}$ mass is probably quite low (at least in the $\eta_{c}$ mass signal region). 

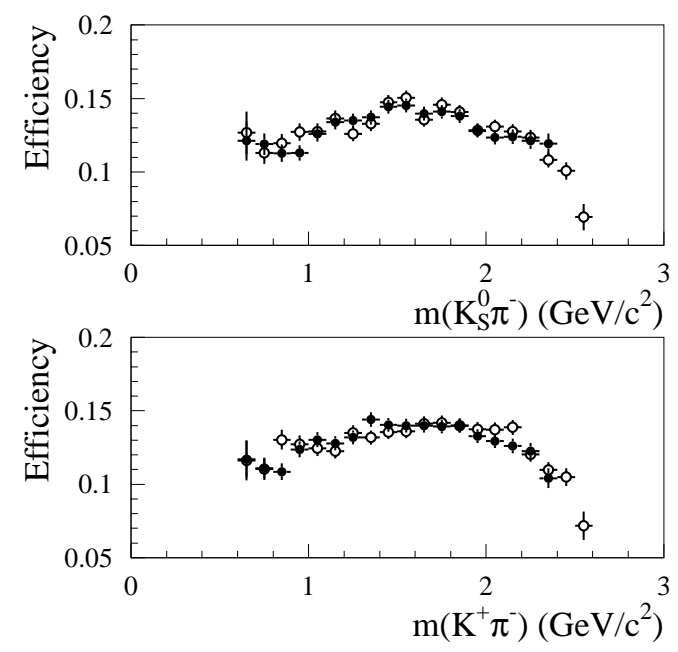

Figure 5.9: Efficiency variation in the $m_{K_{S}^{0} \pi^{-}}$and $m_{K \pi}$ variables in PravdaMC $B^{0} \rightarrow \eta_{c} K_{S}^{0}, \eta_{c} \rightarrow K_{S}^{0} K^{+} \pi^{-}$where the generated $\eta_{c}$ mass is fixed to 2.90 GeV/c (filled circles) and $3.05 \mathrm{GeV} / \mathrm{c}^{2}$ (hollow circles).

\subsection{Monte Carlo Efficiency Corrections}

The SP4 Monte Carlo used to measure the selection efficiency for $B \rightarrow \eta_{c} K, \eta_{c} \rightarrow$ $K \bar{K} \pi$ events may differ from real event data in several ways, since basic detector quantities may be inaccurately modelled in the simulation. The following sections describe these data-Monte Carlo differences, and methods used to account for them. The resulting errors on the measured Monte Carlo efficiency are also discussed. Many of the methods have evolved from independent studies carried out by specific working groups or 'task forces' at BABAR. These methods were used in other previous analyses; for example, the analyses of exclusive charmonium states from $B$ decays [54]. The 'self-combinatorial background' efficiency correction described below is not based on data-Monte Carlo differences but is included alongside them. 


\subsubsection{Tracking Efficiency Corrections}

A tracking efficiency task force [55] at BABAR has studied the efficiency of track reconstruction in data and Monte Carlo. For the category of tracks which demand a minimum number of DCH hits, the efficiency was estimated by counting the total number of tracks reconstructed in the SVT and comparing to the number which were also reconstructed in the $\mathrm{DCH}$.

This method was applied to both Monte Carlo and data, and the relative efficiency (as a function of track momentum, polar angle, and track multiplicity) was determined for each of the the three voltage levels at which the DCH has operated (see Section 2.4).

The discrepancies between Monte Carlo and data in general were found to be very small, with the Monte Carlo overestimating the average track reconstruction efficiency slightly $(0.8 \%)$ compared to real event data, for tracks in the category described above. The task force therefore recommended a factor of 0.992 to be applied to the Monte Carlo selection efficiency for a given analysis, for each track in the category described above. The systematic error on this correction was estimated as $3.5 \%$ per track.

Only the fast kaon candidates in the $B^{+} \rightarrow \eta_{c} K^{+}$channels use the category of tracks described above (see Section 4.4). For tracks which do not demand DCH hits (such as those used to reconstruct the $K_{S}^{0} \rightarrow \pi^{+} \pi^{-}$and $\eta_{c} \rightarrow K \bar{K} \pi$ candidates) the reconstruction efficiency was studied by the task force mentioned above using $\tau$ decays in simulated and real event data. No significant discrepancy was found between data and Monte Carlo, up to a systematic error of $1.3 \%$ per track.

The resulting systematic errors on the $B \rightarrow \eta_{c} K$ Monte Carlo efficiencies arising from the multiple tracks used in reconstruction are given in Table 5.4. 


\subsection{2 $K_{S}^{0}$ Efficiency Corrections}

The task force described in Section 5.3.1 also examined the $K_{S}^{0}$ reconstruction efficiency in data and Monte Carlo, using an inclusive $K_{S}^{0}$ sample [56].

The $K_{S}^{0}$ reconstruction efficiency was measured as a function of the two-dimensional flight length (in the $x, y$ plane). The relative efficiency between data and Monte Carlo was determined separately for each of the three DCH voltage levels. Figure 5.10 shows the relative data-Monte Carlo efficiency at a DCH voltage of $1930 \mathrm{~V}$.
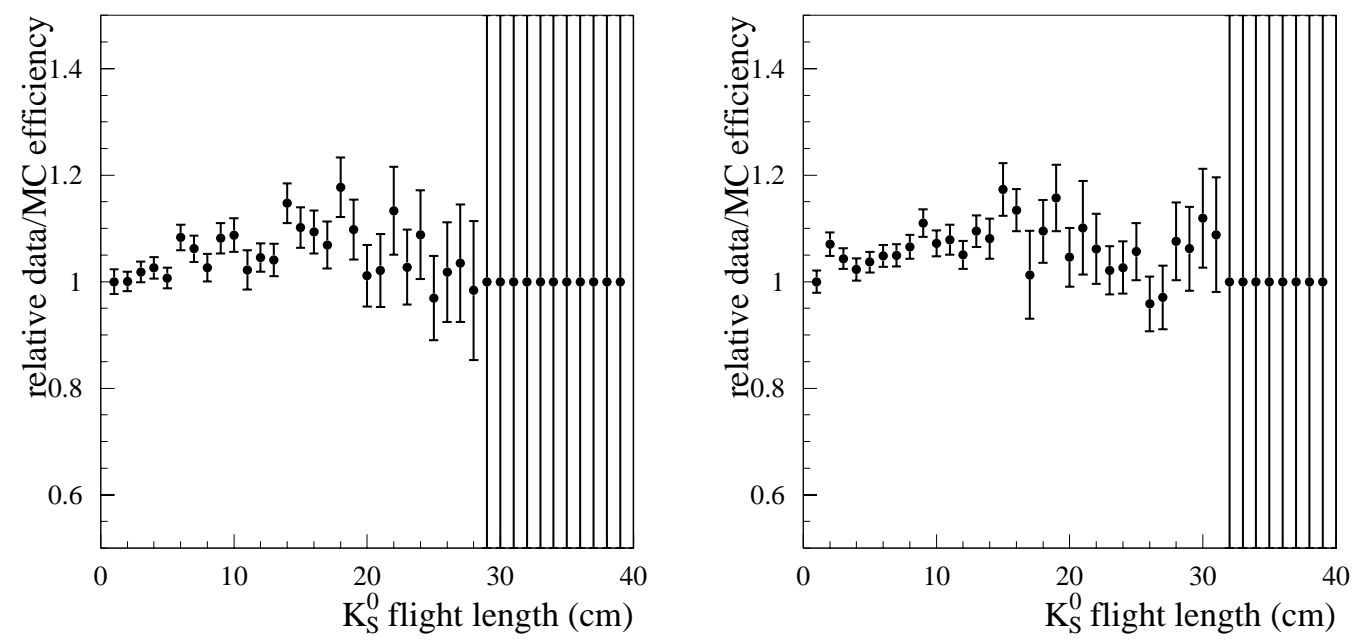

Figure 5.10: The relative data-Monte Carlo $K_{S}^{0}$ reconstruction efficiency for $1930 \mathrm{~V}$ DCH conditions. In the plot on the right the $K_{S}^{0}$ candidates are required to have a minimum transverse momentum of at least $1.0 \mathrm{GeV/c}$.

The relative data-Monte Carlo efficiency in each bin of $K_{S}^{0}$ flight length was measured with respect to that for $K_{S}^{0}$ flight lengths of less than $1 \mathrm{~cm}$. Thus the absolute $K_{S}^{0}$ efficiency corrections are obtained by multiplying the $K_{S}^{0}$ data-Monte Carlo relative efficiencies by the track reconstruction efficiency corrections relevant to the $K_{S}^{0}$ daughter pions. However, since the $K_{S}^{0}$ candidates in this analysis are reconstructed with tracks requiring no efficiency correction, only the $K_{S}^{0}$ efficiency corrections 
themselves are applied.

The efficiency comparisons were determined in one sample with minimal selections (sample A), and in another sample with a requirement that the transverse momentum $\left(p_{T}\right)$ of the $K_{S}^{0}$ candidates exceed $1.0 \mathrm{GeV} / c$ (sample B). In the later sample the extraction of the efficiencies is 'cleaner' in that poorly reconstructed $K_{S}^{0}$ with low transverse momenta are removed.

The Monte Carlo was found in general to underestimate the $K_{S}^{0}$ reconstruction efficiency in data. Thus the selection efficiency in, for example $B^{0} \rightarrow \eta_{c} K_{S}^{0}, \eta_{c} \rightarrow$ $K^{+} K^{-} \pi^{0}$, is corrected by the average relative efficiency of $K_{S}^{0}$ reconstruction in data with respect to Monte Carlo; the average is performed over the sample of $K_{S}^{0}$ flight lengths in the $B^{0} \rightarrow \eta_{c} K_{S}^{0}, \eta_{c} \rightarrow K^{+} K^{-} \pi^{0}$ events after all selections (where $N$ candidates survive). The efficiency correction factor is thus given by

$$
f_{C}=\frac{1}{N} \sum_{i}^{N_{b i n s}} n_{i} c_{i},
$$

where the sum runs over the bins of $K_{S}^{0}$ flight length. $n_{i}$ is the number of $K_{S}^{0}$ (from the $B^{0} \rightarrow \eta_{c} K_{S}^{0}, \eta_{c} \rightarrow K^{+} K^{-} \pi^{0}$ sample) in bin $i$, and $c_{i}$ is the relative data-Monte Carlo efficiency for bin $i$.

To obtain the central value of the correction, the corrections from sample B are applied. The correction is computed for each of the three DCH voltage subsets of the $B \rightarrow \eta_{c} K, \eta_{c} \rightarrow K^{+} K^{-} \pi^{0}$ signal Monte Carlo; the final correction is then given by the luminosity-weighted average of the three corrections, (the weights are the fractions of real data luminosity collected at the three DCH voltages).

The systematic error on the correction is obtained from the difference obtained using sample A. The systematic error is large compared to the statistical error (which arises from the statistical error on the $c_{i}$ values, and is less than $1 \%$ per kaon). Therefore the statistical errors are ignored. 
If there are two $K_{S}^{0}$ are present per event, as in $B^{0} \rightarrow \eta_{c} K_{S}^{0}, \eta_{c} \rightarrow K_{S}^{0} K^{+} \pi^{-}$, the efficiency corrections are computed for each $K_{S}^{0}$ and then multiplied to obtain the final correction. The systematic errors are then added linearly to obtain the total error.

The resulting efficiency correction for $B^{0} \rightarrow \eta_{c} K_{S}^{0}, \eta_{c} \rightarrow K^{+} K^{-} \pi^{0}$ was found to be $1.060 \pm 0.025$. The correction for $B^{0} \rightarrow \eta_{c} K_{S}^{0}\left(B^{+} \rightarrow \eta_{c} K^{+}\right), \eta_{c} \rightarrow K_{S}^{0} K^{+} \pi^{-}$was found to be $1.120 \pm 0.058(1.050 \pm 0.029)$.

\subsection{3 $\pi^{0}$ Efficiency Corrections}

A BABAR working group has studied $\pi^{0}$ reconstruction in data and Monte Carlo, comparing $\pi^{0}$ efficiency and mass spectra [57].

The relative data-Monte Carlo efficiency of $\pi^{0}$ reconstruction has been studied for the reconstructed decays $\tau \rightarrow \pi^{0} \nu_{\tau} X$ and $\tau \rightarrow \pi^{0} \pi^{0} \nu_{\tau} X$ decays, where the ratio of the two yields is sensitive to the $\pi^{0}$ efficiency. The relative size of the ratio in data and Monte Carlo, computed as a function of $\pi^{0}$ energy, gives the relative efficiency of $\pi^{0}$ reconstruction in data with respect to Monte Carlo.

The photon energy scale and resolution was also compared in data and Monte Carlo by fits to the reconstructed $\pi^{0} \rightarrow \gamma \gamma$ mass peak. Poorer resolution in the data can be more closely replicated in the Monte Carlo by energy smearing, in which a Gaussian correction is applied to the photon energies.

Efficiency corrections (so-called $\pi^{0}$ killing) and energy smearing algorithms are implemented in the BABAR software and were activated in the Monte Carlo reconstruction before the preselection. The error involved in applying these corrections can be evaluated by repeating the efficiency correction procedure with the killing and 
smearing parameters adjusted by their errors. The resulting difference in the final selection efficiency is taken as the statistical error on the $\pi^{0}$ killing. This is added in quadrature to a $5 \%$ systematic error (recommended by the $\pi^{0}$ working group) to take into account differences in photon reconstruction efficiency between data and Monte Carlo.

The total Monte Carlo efficiency errors due to $\pi^{0}$ simulation were therefore found to be $5.4 \%$ (5.1\%) for the $B^{0} \rightarrow \eta_{c} K_{S}^{0}\left(B^{+} \rightarrow \eta_{c} K^{+}\right), \eta_{c} \rightarrow K^{+} K^{-} \pi^{0}$ channel.

\subsubsection{Particle Identification Efficiency Corrections}

The efficiency of the kaon identification selectors (see Section 4.5.5) in data and Monte Carlo has been studied by the Particle Identification (PID) Working Group using control samples [48]. The decays $K_{S}^{0} \rightarrow \pi \pi$ and $D^{*} \rightarrow D^{0} \pi, D^{0} \rightarrow K \pi$ provide pure samples of pions and kaons with which to measure kaon selection efficiency and misidentification rates of pions.

The kaon identification efficiency in the $B \rightarrow \eta_{c} K$ Monte Carlo was adjusted to match exactly (to within statistical errors) that observed in the data control samples (measured as a function of track momentum and polar angle). The corrections (socalled PID killing) are implemented in the BABAR software and were applied before the preselection stage.

The errors in the method are thought to arise primarily from differences in the kinematics of the $D^{*}$ and $B \rightarrow \eta_{c} K$ samples, and can be probed by comparing the kaon selector efficiencies in $D^{*}$ and $B \rightarrow \eta_{c} K$ Monte Carlo samples (where no PID killing has been applied) as a function of momentum, using truth-matched candidates $^{1}$. Figure 5.11 shows the efficiency of the Not-a-pion selection on the

\footnotetext{
${ }^{1}$ Carried out by N. Barlow (Charmonium Working Group).
} 
kaons from the $D^{*}$ Monte Carlo and slow kaons in the $B \rightarrow \eta_{c} K, \eta_{c} \rightarrow K_{S}^{0} K^{+} \pi^{-}$ Monte Carlo.

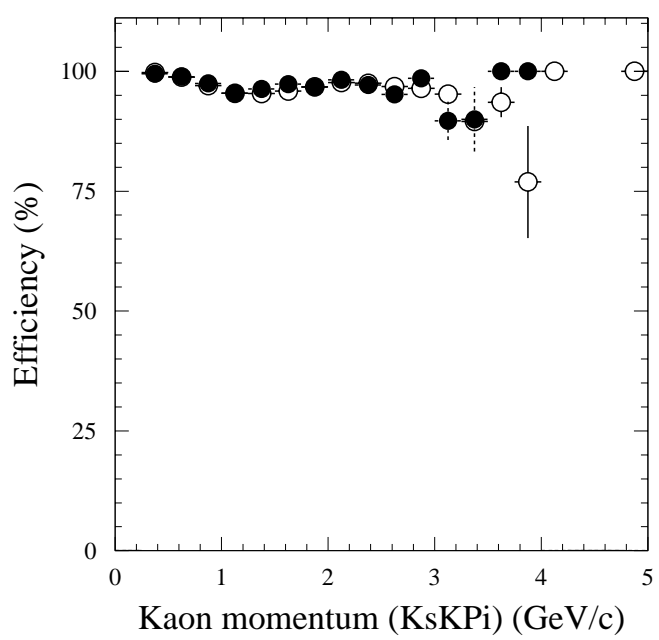

Figure 5.11: Efficiency of 'Not a pion' kaon identification for truth-matched kaons from $D^{*}$ Monte Carlo (hollow circles) and slow kaons from $B \rightarrow \eta_{c} K$, $\eta_{c} \rightarrow K_{S}^{0} K^{+} \pi^{-}$Monte Carlo (filled circles). This plot was produced by $N$. Barlow (Charmonium Working Group).

A weighted average efficiency difference between the $D^{*}$ and $B \rightarrow \eta_{c} K$ Monte Carlo was obtained for each kaon candidate to which particle identification was applied. The average was performed using the relative efficiency difference in each momentum bin over the whole kaon momentum range, weighting by the fractional occupancy (of the kaons from the $B \rightarrow \eta_{c} K$ sample) in each momentum bin.

When comparing the $B \rightarrow \eta_{c} K$ fast kaon selection, a systematically lower efficiency was observed for the kaons in the $D^{*}$ sample; this is thought to be due to differences in the angular and momentum distributions between the two kaons samples. This suggests that the corrected efficiency for the fast kaons is too low, and an overall correction of $+4.7 \%$ is applied to account for this, with an error of $2.35 \%$. 
There is also a statistical error involved in the PID killing procedure, due to the finite $D^{*}$ sample size; however, the statistical error is almost negligible compared to the systematic error described above, to which it is added in quadrature.

In the Run 1 analysis small systematic errors associated with the method of measuring the kaon identification efficiency were also included [58]. The efficiency errors were estimated at $\sim 1.5 \%$ per kaon. However these errors were not re-evaluated by the PID working group for data-SP4 Monte Carlo PID study. Since fully reliable estimates do not exist for these (probably small) errors for the present data sample, they are neglected here.

The final efficiency errors were evaluated at $0.4 \%(2.8 \%)$ for $B^{0} \rightarrow \eta_{c} K_{S}^{0}\left(B^{+} \rightarrow\right.$ $\left.\eta_{c} K^{+}\right), \eta_{c} \rightarrow K_{S}^{0} K^{+} \pi^{-}$and $2.6 \%(5.0 \%)$ for $B^{0} \rightarrow \eta_{c} K_{S}^{0}\left(B^{+} \rightarrow \eta_{c} K^{+}\right), \eta_{c} \rightarrow$ $K^{+} K^{-} \pi^{0}$.

\subsection{5 $\eta_{c}$ Width Uncertainty}

The simulated width of the $\eta_{c}$ in Monte Carlo directly affects the measured selection efficiency, since only $\eta_{c}$ candidates within $70 \mathrm{MeV} / c^{2}$ of the nominal $\eta_{c}$ mass are accepted. An underestimation (overestimation) of the $\eta_{c}$ width in the Monte Carlo leads to an overestimation (underestimation) of the average selection efficiency.

As mentioned (Section 4.5.8), the $\eta_{c}$ width in the Monte Carlo used for this analysis was fixed to the best single measurement available (at the time that the Monte Carlo was produced). Recent results from $B \rightarrow \eta_{c} K$ events including those from this analysis (see Section 5.6.1) and two-photon production of the $\eta_{c}$ have indicated that the $\eta_{c}$ width is $\gtrsim 30 \mathrm{MeV} / c^{2}$, and that the present world average underestimates the $\eta_{c}$ width (see Section 1.6.2). It therefore seems reasonable to take the measurement of $\Gamma\left(\eta_{c}\right)=27 \pm 6 \mathrm{MeV} / c^{2}$ as a guide to the possible range of the $\eta_{c}$ width. 
The sensitivity of the efficiency variation in the $\eta_{c}$ decay phase space to the $\eta_{c}$ width is probably quite low, since the efficiency variation is not seen to change greatly as a function of $\mathrm{m}\left(\eta_{c}\right)$, within the $\eta_{c}$ signal region (see Section 5.2.5); the effect will probably be second order compared to the effect on the average efficiency.

The effect of the $\Gamma\left(\eta_{c}\right)$ uncertainty on the average efficiency can be estimated by rescaling the $\eta_{c}$ width in the Monte Carlo during the selection procedure. This is possible since the generated and reconstructed $\eta_{c}$ mass is available for each candidate; the resolution contribution to each candidate can be separated and added to the rescaled generated mass value. However, the momenta of the daughter particles cannot be altered accordingly.

Following the procedure above to rescale the generated $\eta_{c}$ width to $33 \mathrm{MeV} / c^{2}$ and $21 \mathrm{MeV} / c^{2}$ gives a rather symmetrical systematic error of $3.0 \%$ to the measured efficiency across all $B \rightarrow \eta_{c} K, \eta_{c} \rightarrow K \bar{K} \pi$ channels. Therefore a systematic error of $3 \%$ is assigned to the selection efficiency for each $B \rightarrow \eta_{c} K, \eta_{c} \rightarrow K \bar{K} \pi$ channel.

\subsubsection{Selection Variable Distributions}

Any discrepancy between the simulated and real distributions of variables which are used to select candidates results in an systematic effect on the Monte Carlo efficiency estimate. For instance in the Run 1 analysis the $K_{S}^{0}$ mass resolution was found to be $30 \%$ poorer in data than in Monte Carlo; thus the selection efficiency was overestimated.

Some of the Monte Carlo corrections described above resolve some of the discrepancies (such as the $\pi^{0}$ smearing and PID killing). However they may not be sufficient to account for all data-Monte Carlo differences. 
In principle, the discrepancies between data and Monte Carlo may be probed by varying the requirement on each selection variable and observing the resulting change in the branching fraction (no change is expected in the case of no discrepancy). This method was investigated in the Run 1 analysis. However, with the statistically limited yields in data, any changes when varying a selection requirement tended to be large and dominated by statistical fluctuations. Although the present analysis uses a data sample approximately four times that of the Run 1 analysis, the expected yields are not sufficiently large to allow the separation of statistical and systematic effects when varying the selections.

Instead a simpler approach was used for the Run 1 analysis. A more general comparison was made between data and Monte Carlo for each selection variable distribution. Each variable distribution was plotted for the data, and for a combination of $e^{+} e^{-} \rightarrow B \bar{B}, u \bar{u}, d \bar{d}, s \bar{s}, c \bar{c}$ (where the proportions in the combination are governed by the estimated cross-section for each type). Any overall discrepancy in each distribution was measured by comparing the binned, integrated distributions. Selections were relaxed to increase the statistical power of the comparison; for example when comparing the $K_{S}^{0}$ mass distributions from $B^{0} \rightarrow \eta_{c} K_{S}^{0}$ candidates, all selections were relaxed except the those on the other $K_{S}^{0}$ discriminating variables, the flight length and $\cos \alpha\left(K_{S}^{0}\right)$.

This approach was used for the present data sets to determine differences between data and Monte Carlo for all selection variables except the Fisher discriminant, the reconstructed $\eta_{c}$ mass, $\Delta E$ and $M_{E S}$. The comparisons can be seen in Figure 5.12. ${ }^{2}$.

For the case of the Fisher variable, a different approach was used since a data control

\footnotetext{
${ }^{2}$ This work was done by N. Barlow (Charmonium Working Group). For the $\eta_{c} \rightarrow K^{+} K^{-} \pi^{0}$ channel a different set of selections was used to the ones described in Chapter 4. The main selection differences included $|\Delta E|<75 \mathrm{MeV}$ and minimum energies of $80 \mathrm{MeV}$ and $120 \mathrm{MeV}$ for the $\pi^{0}$ candidate low and high energy photon daughters respectively.
} 


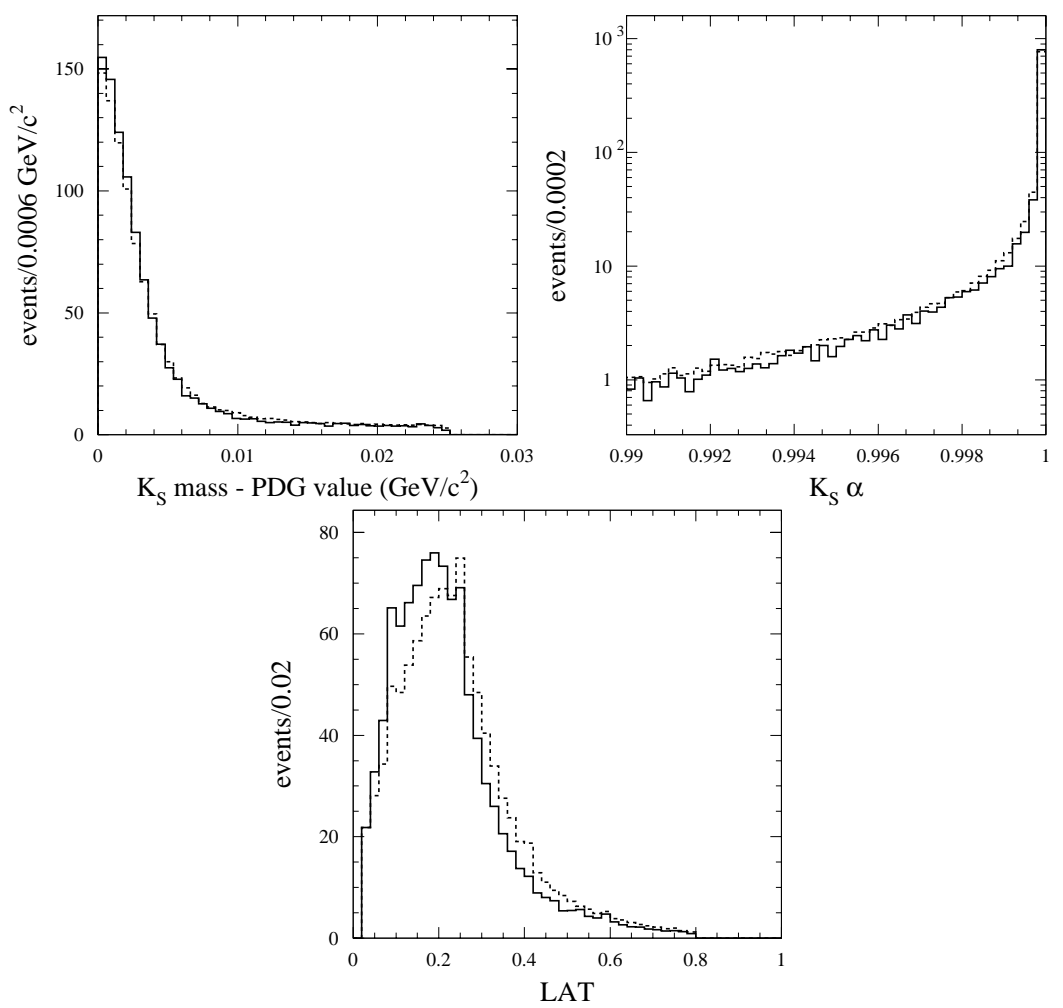

Figure 5.12: Comparison of selection variable distributions in data (dashed line) and Monte Carlo (solid line). The $K_{S}^{0}$ mass (' $K_{S}$ mass - PDG value'), $\cos \alpha\left(K_{S}^{0}\right)$ (' $K_{S} \alpha$ ') are shown for fast kaon candidates. The photon ( $\pi^{0}$ daughter) lateral moment is also shown. These plots were produced by N. Barlow (Charmonium Working Group).

sample of $B \rightarrow J / \Psi K$ events was available. The Fisher distribution from the control sample was directly compared to that from $B \rightarrow J / \Psi K$ Monte Carlo (see Figure 5.13). Again, the integral distributions gave a measure of the discrepancy.

The general level of agreement seen in Monte Carlo and data in the Fisher discriminant and the other variables is excellent. Integrals of the binned distributions shown in Figure 5.12 give a discrepancy at the $\lesssim 1 \%$ level at the selection values used, expect for the $\cos \alpha\left(K_{S}^{0}\right)$ for the fast $K_{S}^{0}$ candidates, for which the discrepancy at the selection value is $2.2 \%$. Since the observed discrepancies are small and likely 


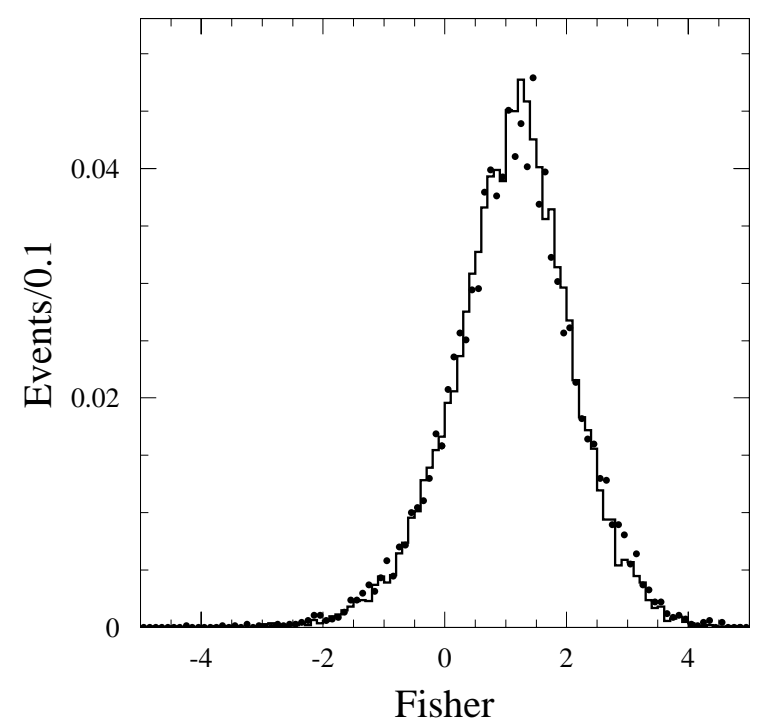

Figure 5.13: Comparison of the Fisher discriminant distributions for $B \rightarrow$ $J / \Psi K$ events, for data control sample and signal Monte Carlo. The Fisher coefficients used were those obtained for the $\eta_{c} \rightarrow K^{+} K^{-} \pi^{0}$ channel. This plot was produced by N. Barlow (Charmonium Working Group).

to be statistical rather than systematic, no systematic errors were assigned for the variables discussed above, except $\cos \alpha\left(K_{S}^{0}\right)$.

For variables specifically related to the $B$ meson $\left(\Delta E\right.$ and $\left.M_{E S}\right)$ the resolutions were measured from the signal yield in data (see Section 5.6), and compared with signal Monte Carlo. Systematic errors resulting from discrepancies in $M_{E S}$ and $\Delta E$ in Monte Carlo and data are described in Section 5.6.3. Systematic errors due to an inaccurate modelling of the $\eta_{c}$ width in Monte Carlo are discussed in Section 5.3.5. 


\subsubsection{Self-Combinatorial Background}

A final correction to the Monte Carlo efficiency was made, not based on Monte-Carlo differences. The efficiency measured using Monte Carlo may potentially be polluted by combinatorial background within simulated $B \rightarrow \eta_{c} K$ events. The amount of self-combinatorial background, after applying all selections may be estimated by fitting the $M_{E S}$ distribution in the $\Delta E$ signal region with the sum of an ARGUS function and a Gaussian ${ }^{3}$. This is illustrated by Figure 5.14 which shows the fit in the $B^{+} \rightarrow \eta_{c} K^{+}$channels. The quality of the ARGUS component of the fit is poor but the distribution alone shows that the combinatorial background in the $M_{E S}$ signal region is very low. Taking the numbers from the fits, $0.5 \%(0.9 \%)$ of the $B^{+} \rightarrow \eta_{c} K^{+}$ candidates in the $\eta_{c} \rightarrow K_{S}^{0} K^{+} \pi^{-}\left(\eta_{c} \rightarrow K^{+} K^{-} \pi^{0}\right)$ channels are attributed to selfcombinatorial background. The equivalent results for $B^{0} \rightarrow \eta_{c} K_{S}^{0}$ candidates are $0.2 \%(0.4 \%)$. The Monte Carlo efficiencies are thus rescaled accordingly (see Table 5.3). Since these are only approximate correction estimates, a systematic error of half the correction is applied; the errors are therefore negligible except perhaps in the $B^{+} \rightarrow \eta_{c} K^{+}, \eta_{c} \rightarrow K^{+} K^{-} \pi^{0}$ channel.

\subsubsection{Summary}

A summary of the Monte Carlo efficiency corrections is given in Table 5.3, while systematic errors from data-Monte Carlo differences is given in Table 5.4. The data-Monte Carlo discrepancies in the $\Delta E$ and $M_{E S}$ distributions are addressed in Section 5.6.3.

\footnotetext{
${ }^{3}$ The use of this fitting function to determine combinatorial background will be discussed in Section 5.4.1.
} 

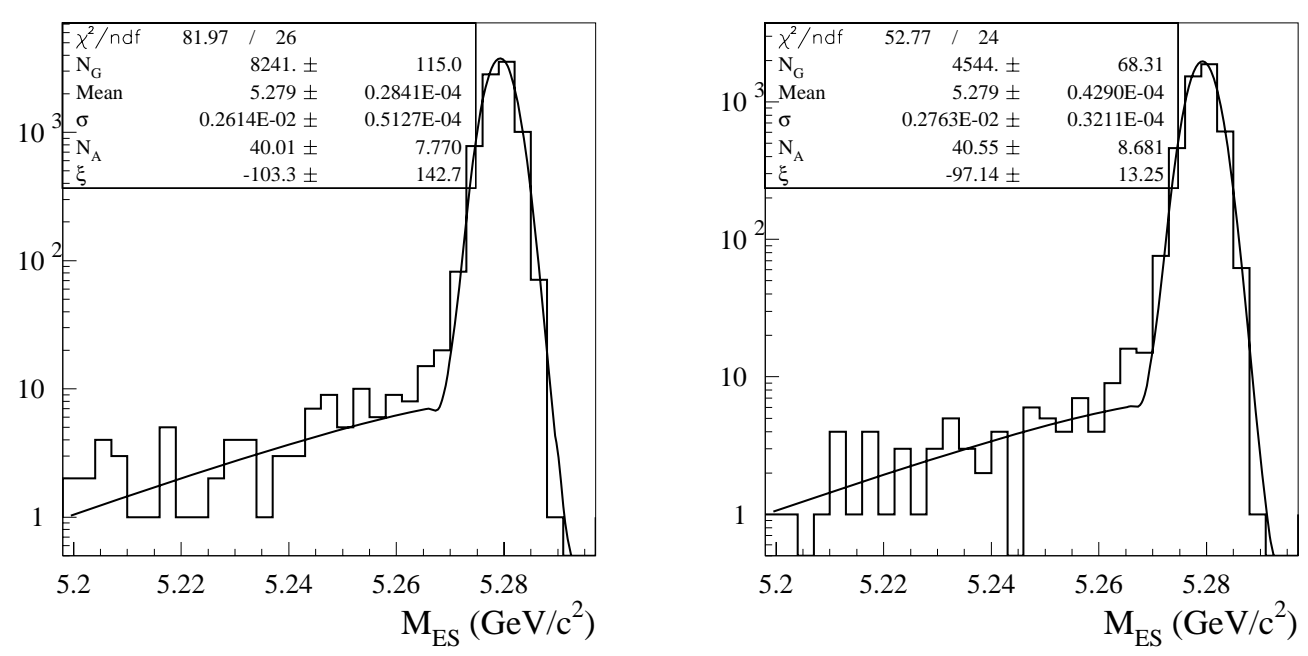

Figure 5.14: $M_{E S}$ distributions (logarithmic vertical scale) for Monte Carlo $B^{+} \rightarrow \eta_{c} K^{+}$candidates, after all selections, for $\eta_{c} \rightarrow K_{S}^{0} K^{+} \pi^{-}$(left) and $\eta_{c} \rightarrow K^{+} K^{-} \pi^{0}$ (right).

Table 5.3: Monte Carlo efficiency correction factors. Possible corrections due to data-Monte Carlo discrepancies in $\Delta E$ and $M_{E S}$ are addressed in Section 5.6.3.

\begin{tabular}{c|c|c|c|c}
\hline \multirow{2}{*}{$\eta_{c}$ Decay Channel } & \multicolumn{2}{|c|}{$K_{S}^{0} K^{+} \pi^{-}$} & \multicolumn{2}{c}{$K^{+} K^{-} \pi^{0}$} \\
\cline { 2 - 5 } & $B^{0}$ & $B^{+}$ & $B^{0}$ & $B^{+}$ \\
\hline \hline Track efficiency & - & 0.992 & - & 0.992 \\
$K_{S}^{0}$ efficiency & 1.12 & 1.05 & 1.06 & - \\
Kaon PID efficiency & - & 1.047 & - & 1.047 \\
Self-combinatorial-background & 0.998 & 0.995 & 0.996 & 0.991 \\
\hline
\end{tabular}

\subsection{Background Estimation}

Background in the selection of $B \rightarrow \eta_{c} K, \eta_{c} \rightarrow K \bar{K} \pi$ candidates was first addressed in the selection optimization procedure (see Chapter 4). The estimated background $N_{B G}$ is an essential ingredient of the branching fraction calculation, Equation (5.1). 
Table 5.4: Relative percentage errors on Monte Carlo efficiency due to data-Monte Carlo discrepancies. Errors due to discrepancies in $\Delta E$ and $M_{E S}$ are addressed in Section 5.6.3.

\begin{tabular}{c|c|c|c|c}
\hline \multirow{2}{*}{$\eta_{c}$ Decay Channel } & \multicolumn{2}{|c|}{$K_{S}^{0} K^{+} \pi^{-}$} & \multicolumn{2}{l}{$K^{+} K^{-} \pi^{0}$} \\
\cline { 2 - 5 } & $B^{0}$ & $B^{+}$ & $B^{0}$ & $B^{+}$ \\
\hline \hline Track efficiency (\%) & 7.8 & 6.3 & 5.2 & 4.4 \\
$K_{S}^{0}$ efficiency (\%) & 5.8 & 2.9 & 2.5 & - \\
Kaon PID efficiency (\%) & 0.4 & 2.8 & 2.6 & 5.0 \\
$\pi^{0}$ efficiency (\%) & - & - & 5.4 & 5.1 \\
$\eta_{c}$ width uncertainty (\%) & 3.0 & 3.0 & 3.0 & 3.0 \\
Fast $K_{S}^{0}$ selection (\%) & 2.2 & - & 2.2 & - \\
Self-combinatorial-background (\%) & 0.1 & 0.3 & 0.3 & 0.5 \\
\hline
\end{tabular}

As mentioned previously, background in the selection of $B \rightarrow \eta_{c} K, \eta_{c} \rightarrow K \bar{K} \pi$ candidates arises primarily from non-signal $B \bar{B}$ and continuum events. The background contributions can be divided into two categories; combinatorial and peaking background. It is preferable to estimate background from the data rather than Monte Carlo, to avoid the systematic errors involved in simulation.

\subsubsection{Combinatorial Background}

In the selection optimisation procedure, only combinatorial background was addressed, and was estimated from the $M_{E S}$ distribution in the $\Delta E$ sideband region using the ARGUS function description, and interpolating to the $\Delta E$ signal band. Although this method gives a reasonable estimate of the combinatorial background, some previous Monte Carlo studies in the Run 1 analysis showed that the shape of the combinatorial background varied as a function of $\Delta E$ [58]. Therefore a system- 
atic effect may be involved when estimating the combinatorial background using the $\Delta E$ sidebands.

A more reliable combinatorial background estimate is given by a fit of the sum of an ARGUS function and a Gaussian to the $M_{E S}$ distribution in the $\Delta E$ signal band rather than the above method. The number of combinatorial background events $N_{B G}^{a r g}$ is directly obtained from the integral of the ARGUS component of the fitted function in the $M_{E S}$ signal region.

\subsubsection{Peaking Background}

There may be an additional background component not described by the ARGUS shape in $M_{E S}$, known as peaking background. The source of peaking background is $B \bar{B}$ decays and may arise in several ways.

There may be cross-feed between $\eta_{c}$ decay modes in $B \rightarrow \eta_{c} K$ decays, for example $\eta_{c} \rightarrow h^{+} h^{-} h^{+} h^{-}$(where $h$ represents a kaon or pion) may be reconstructed as a $\eta_{c} \rightarrow K_{S}^{0} K^{+} \pi^{-}$candidate. Similarly there may be cross-feed in the $\eta_{c} \rightarrow K^{+} K^{-} \pi^{0}$ channel from the $\eta_{c} \rightarrow \eta \pi^{+} \pi^{-}$channel. However, the constraints in reconstruction ( $K_{S}^{0}$ selection, kaon identification) highly suppress cross-feed background. This was studied in the Run 1 analysis. Thousands of exclusive signal Monte Carlo decays of the types above were produced; the yields from potential cross-feed modes was found to be negligible for all $\eta_{c}$ decay modes [58]. Inclusive $B \rightarrow \eta_{c} X$ modes can also contribute; however a similar Monte Carlo study to the one described above found the background to be negligible in this category [58].

Another possibility is partial reconstruction of $B$ decays containing the same final state particles as the $B \rightarrow \eta_{c} K$ final state. An example of this would be the partial reconstruction of $B^{0} \rightarrow D^{+} K^{-} K^{0}, D^{+} \rightarrow K^{0} \pi^{+} \pi^{0}$ as a $B^{0} \rightarrow \eta_{c} K_{S}^{0}, \eta_{c} \rightarrow K_{S}^{0} K^{+} \pi^{-}$ 
candidate.

Lastly, peaking background may arise from $B$ decays to the same final states as those from the $B \rightarrow \eta_{c} K$ decays. These may include non-resonant $B$ decays or those through other resonances. This class of peaking background may include $B \rightarrow J / \Psi K$ decays where the $J / \psi$ decays to the same channel as the $\eta_{c}$, if the allowed range of reconstructed $\eta_{c}$ masses is sufficiently large.

Using a large sample of generic $B \bar{B}$ decays (generated with PravdaMc) the amounts of peaking background from partial reconstruction and exact matches were studied ${ }^{4}$. For $\eta_{c} \rightarrow K_{S}^{0} K^{+} \pi^{-}$the background events after all selections were found to be dominated by exact matches (see Table 5.5). It should be noted that this study cannot give an reliable quantitative measure of the expected amount of peaking background, since the proportions of the contributing decay modes are governed by the Monte Carlo input branching fractions, which are poorly measured by experiment, and in some cases unknown.

In the Run 1 data sample the peaking background measurement was statistically limited. No significant amount of peaking background was found in the $\eta_{c} \rightarrow K^{+} K^{-} \pi^{0}$ channels, while in the $B^{+} \rightarrow \eta_{c} K^{+}, \eta_{c} \rightarrow K_{S}^{0} K^{+} \pi^{-}$channel it was estimated that peaking background accounted for $8.5 \pm 3.6 \%$ of the total yield in the $M_{E S}, \Delta E$ signal region [37].

The peaking background estimation in the Run 1 analysis used the $\eta_{c}$ mass distribution. It was assumed that events contributing to peaking background should exhibit no particular structure in the $\eta_{c}$ mass distribution, since no $\eta_{c}$ is present in those events. Therefore the excess of events in the $\Delta E, M_{E S}$ signal region, not described by the ARGUS function, for events that fall in the $\eta_{c}$ mass sidebands can be extrapolated to the amount of peaking background in the $\eta_{c}$ mass signal band.

\footnotetext{
${ }^{4}$ This study was performed by S. Riccardi (Charmonium Working Group).
} 
Table 5.5: Percentage of signal and background types after all selections in generic $B \bar{B}$ PravdaMC sample.

\begin{tabular}{c|c|c}
\hline \multirow{2}{*}{$\eta_{c}$ Decay Channel } & \multicolumn{2}{|c}{$K_{S}^{0} K^{+} \pi^{-}$} \\
\cline { 2 - 3 } & $B^{0}$ & $B^{+}$ \\
\hline \hline Signal Events (\%) & 63.0 & 71.0 \\
Exact Matches (\%) & 15.8 & 18.6 \\
Partial Reconstruction (\%) & 4.4 & 2.9 \\
Combinatorial (\%) & 3.5 & 4.4 \\
Other (\%) & 13.2 & 3.1 \\
\hline
\end{tabular}

The $\eta_{c}$ mass sidebands in the Run 1 analysis were defined such that they contained negligible numbers of real $B \rightarrow \eta_{c} K$ or $B \rightarrow J / \Psi K$ events. Sidebands defined by $2.7 \mathrm{GeV} / c^{2}<m\left(\eta_{c}\right)<2.85 \mathrm{GeV} / c^{2}, 3.15 \mathrm{GeV} / c^{2}<m\left(\eta_{c}\right)<3.3 \mathrm{GeV} / c^{2}$ hold approximately approximately $2 \%$ of truth-matched $B \rightarrow \eta_{c} K$ events in SP4 Monte Carlo $\left(\right.$ with $\left.\Gamma\left(\eta_{c}\right)=27 \mathrm{MeV} / c^{2}\right)$.

In practice, the total yield $\left(N_{Y}^{s b}\right)$ in the $\Delta E, M_{E S}$ signal region, subtracted by the combinatoric background ( $N_{C}^{s b}$, estimated by the ARGUS function), was evaluated for $\eta_{c}$ candidates falling in the $\eta_{c}$ sidebands, as defined above. The result was then scaled to the $\eta_{c}$ mass signal region by the ratio $(R)$ of width of the signal region to that of the sidebands. The peaking background estimate can thus be represented by

$$
N_{B G}^{p k}=R \cdot\left(N_{Y}^{s b}-N_{C}^{s b}\right) .
$$




\subsubsection{Background Subtraction}

The background-subtracted yield (the numerator in (5.1)), accounting for peaking and combinatorial background is given by

$$
N_{Y}-N_{B G}^{a r g}-R \cdot\left(N_{Y}^{s b}-N_{C}^{s b}\right),
$$

where $N_{Y}, N_{B G}^{a r g}, R, N_{Y}^{s b}, N_{C}^{s b}$ are defined in the previous sections. It is worth noting that the combinatorial background, as well as the peaking background may be expected to be uniform in reconstructed $\eta_{c}$ mass. In that case, $N_{B G}^{a r g} \approx R . N_{C}^{s b}$ and the total background could be estimated simply by the yield in the $\eta_{c}$ mass sidebands; thus the background-subtracted yields would become

$$
N_{Y}-R \cdot N_{Y}^{s b} .
$$

The assumptions concerning the shape of the combinatorial and peaking background in the $\eta_{c}$ mass distribution, and a method of subtracting the background when using efficiency-weighted candidates were tested using the generic $B \bar{B}$ Monte Carlo sample mentioned in Section 5.4.2. This is discussed in the next section.

\subsubsection{Validation of Background Subtraction}

The measurement of the background-subtracted yield using a sample of efficiencyweighted $B \rightarrow \eta_{c} K$ candidates (see Section 5.2), is rather different to the efficiencycorrected yield measurement where candidates are not weighted. In the latter case the background may be subtracted before the efficiency correction is applied, (as is demonstrated by (5.1)). In principle, as long as the weighting procedure does not bias the shape of the backgrounds over the regions of extrapolation, the background can be subtracted after the weights have been applied. 
To validate the background estimation using a weighted data sample, a Monte Carlo (PravdaMC) study was performed. The Monte Carlo sample was composed of the generic $B^{0} \bar{B}^{0}$ sample described in the previous section (having removed all events containing $B \rightarrow \eta_{c} K$ decays), combined with a certain number $\left(N_{S}\right)$ of separately generated signal $B \rightarrow \eta_{c} K, \eta_{c} \rightarrow K_{S}^{0} K^{+} \pi^{-}$events. The calculated backgroundsubtracted yield using this sample should be equal to the expected result $\left(N_{S}\right)$, to within statistical errors.

The generic $B^{0} \bar{B}^{0}$ sample contained $277,330,000$ events. The expected number of $B^{0} \rightarrow \eta_{c} K_{S}^{0}, \eta_{c} \rightarrow K_{S}^{0} K^{+} \pi^{-}$events within a sample of this size is approximately 1,270 (using the $B \rightarrow \eta_{c} K$ branching fractions [37]). Thus $N_{S}$ was chosen to be 1,270, although this choice is not strictly necessary to the validation of the background subtraction method. To compute the signal efficiency parametrization, a statistically independent sample of $50,000 B^{0} \rightarrow \eta_{c} K_{S}^{0}$ events was used.

All the analysis selections were applied, including the additional requirement on the slow pion momentum in $\eta_{c} \rightarrow K_{S}^{0} K^{+} \pi^{-}$described in Section 5.2.4. Without this extra selection, the resulting efficiency or weight distribution is narrow, and the distributions in $M_{E S}, m\left(K_{S}^{0} K^{+} \pi^{-}\right)$would effectively be trivially rescaled by a constant factor when the weights are applied.

Figure 5.15 shows the counted yield in the $\Delta E, M_{E S}$ signal region as a function of $m\left(K_{S}^{0} K^{+} \pi^{-}\right)$for the Monte Carlo sample described above, with the estimated combinatorial background superimposed, for both weighted and unweighted samples. The peaking and combinatorial background are observed to be approximately uniform in $m\left(K_{S}^{0} K^{+} \pi^{-}\right)$. The peak at around $3.1 \mathrm{GeV} / c^{2}$ in $m\left(K_{S}^{0} K^{+} \pi^{-}\right)$is due to $B \rightarrow J / \Psi K, J / \psi \rightarrow K_{S}^{0} K^{+} \pi^{-}$events in the $B \bar{B}$ sample.

The efficiency-corrected signal yield was computed for the weighted and unweighted samples. For the unweighted samples the efficiency-corrected yield was obtained by 

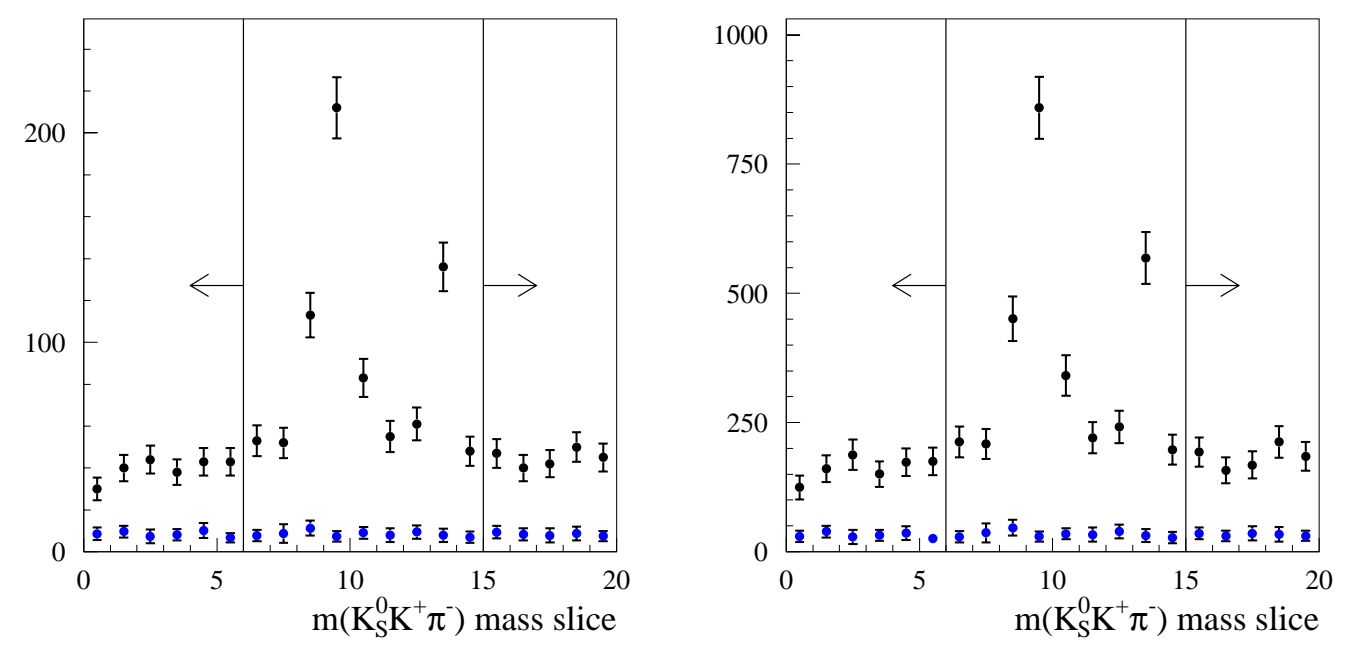

Figure 5.15: Total yield (black) and estimated combinatorial background (blue) as a function of $m\left(K_{S}^{0} K^{+} \pi^{-}\right)$for unweighted sample (left) and weighted sample (right). The horizontal axis has the range $2.7 \mathrm{GeV} / \mathrm{c}^{2}<$ $m\left(K_{S}^{0} K^{+} \pi^{-}\right)<3.3 G e V / c^{2}$, divided into 20 slices. The arrows denote the sideband regions.

dividing the yield after combinatorial and peaking background subtraction by the average selection efficiency. For the weighted sample the efficiency-corrected yield was obtained simply by the yield after background subtraction.

The combinatorial background can be estimated in two ways; using the sum of ARGUS and Gaussian fit in the $m\left(K_{S}^{0} K^{+} \pi^{-}\right)$(method A), or in the $m\left(K_{S}^{0} K^{+} \pi^{-}\right)$ sidebands followed by linear extrapolation to the $m\left(K_{S}^{0} K^{+} \pi^{-}\right)$signal region (method B). Method A gives $N_{B G}^{a r g}$ while method B gives $R . N_{C}^{s b}$ (see Section 5.4.3). The difference in the results obtained by the two methods may be caused by statistical nonuniformites in the background over the $m\left(K_{S}^{0} K^{+} \pi^{-}\right)$range and/or systematic errors involved in the fits.

The results are shown in Tables 5.6 and 5.7. For both the weighted and unweighted 
samples the efficiency-corrected signal yield is consistent with the expected result $(1,270)$, although all three results are about $1 \sigma(8 \%)$ low. This may be partly due to the binomial error on the surviving candidates from the signal $B^{0} \rightarrow \eta_{c} K_{S}^{0}$ events (approximately 5\% error on a sample of 1,270 generated events with estimated $24.7 \%$ selection efficiency), and/or a statistical or systematic over-estimation of the background. A more detailed study would require a large number of statistically independent simulated signal and background samples to be generated; repeated measurements would reveal any systematic misestimations of the background.

Table 5.6: Efficiency-corrected yield (ECY) for the unweighted Monte Carlo sample described in the text. The two methods of evaluating the combinatorial background are described in the text. The final ECY is computed using method A.

\begin{tabular}{c|c}
\hline$\eta_{c}$ Decay Channel & $K_{S}^{0} K^{+} \pi^{-}\left(B^{0}\right)$ \\
\hline \hline Total yield & $497 \pm 22.3$ \\
Combinatorial background (method A) & $26.3 \pm 4.2$ \\
Combinatorial background (method B) & $18.7 \pm 2.4$ \\
Peaking background & $176.8 \pm 9.2$ \\
Signal yield (method A) & $293.8 \pm 24.5$ \\
Efficiency & $24.7 \%$ \\
ECY (method A) & $1189.5 \pm 99.2$ \\
\hline
\end{tabular}

Since the agreement observed here is at the $1 \sigma$ level, no large systematic errors are obvious. It is thus possible to conclude that the method of subtracting peaking and combinatorial background is valid for $B \bar{B}$ Monte Carlo, using weighted data samples. In real data, the combinatorial background is expected to much higher due to contributions from continuum events. Although the combinatorial background in this study appears uniform in $\mathrm{m}\left(\eta_{c}\right)$, the estimate from method $\mathrm{A}$ and method $\mathrm{B}$ are not consistent within $1 \sigma$ statistical error. However it is difficult to conclude a systematic effect based on the above study that will be relevant to the full data 
Table 5.7: Efficiency-corrected yield (ECY) for the weighted Monte Carlo sample described in the text. Weights from the efficiency parametrizations using the $K_{S}^{0} \pi$ and $K \pi$ systems are used, with the results for the latter given in parenthesis.

\begin{tabular}{c|c}
\hline$\eta_{c}$ Decay Channel & $K_{S}^{0} K^{+} \pi^{-}\left(B^{0}\right)$ \\
\hline \hline Total yield & $2007.7 \pm 92.0(2022.4 \pm 92.7)$ \\
Combinatorial background (method A) & $104.9 \pm 16.9(109.7 \pm 17.0)$ \\
Combinatorial background (method B) & $72.0 \pm 9.8(70.7 \pm 9.7)$ \\
Peaking background & $726.7 \pm 40.9(752.1 \pm 42.4)$ \\
ECY (method A) & $1176.1 \pm 102.1(1160.6 \pm 103.4)$ \\
\hline
\end{tabular}

sample since the combinatorial and peaking background proportions will be quite different due to continuum events.

\section{5 $B$ Meson Counting}

Another vital ingredient of the branching fraction calculation (5.1) is the total number of $B$ mesons in the data sample.

A 'luminosity' working group at BABAR provides tools for any given analysis to calculate the number of $B \bar{B}$ pairs in its individual data sample [59]. The $B$ counting method calculates the number of $\Upsilon(4 S)$ mesons produced (the $\Upsilon(4 S) \rightarrow B^{0} \bar{B}^{0}$ and $\Upsilon(4 S) \rightarrow B^{+} B^{-}$branching fractions are assumed to be equal), which is probed by the increase in the ratio of the number of hadronic events to muon pairs in onresonance data compared to off-resonance data. The number of $B \bar{B}$ pairs for a given on-resonance dataset is

$$
N_{B \bar{B}}=\left(N_{M H}^{O N}-N_{M H}^{O F F}\right) / \epsilon_{B \bar{B}},
$$


where $N_{M H}^{O N}$ is the measured number of multihadronic events in the dataset, $N_{M H}^{O F F}$ is the expected number of multihadronic events for an off-resonance dataset of equivalent integrated luminosity, and $\epsilon_{B \bar{B}}$ is the efficiency for $B \bar{B}$ events to pass the multihadronic event selection. $N_{M H}^{O F F}$ is estimated using the measured number of muon pairs in the on-resonance dataset multiplied by the ratio of the number of multihadronic events to muon pairs in off-resonance data. $\epsilon_{B \bar{B}}$ is measured with Monte Carlo.

The $N_{B \bar{B}}$ calculations are done on a run-by-run basis and the information is stored in the $B A B A R$ database, where it can be retrieved and summed over the runs used in a specific analysis.

For the 'good-quality' runs used in the data sample of this analysis (see Section 4.2),

$$
N_{B \bar{B}}=86,186,000 \pm 948,000
$$

was obtained using the tools provided by the luminosity group.

\subsection{Branching Fractions}

\subsubsection{Signal Observations and Preliminary Yields}

Applying the selections described in Chapter 4 to the whole $79.4 \mathrm{fb}^{-1}$ data sample, the following $B \rightarrow \eta_{c} K$ signals and yields are obtained.

The two dimensional plots of $M_{E S}$ vs. $\Delta E$ (combined $B^{0}$ and $B^{+}$samples) are shown in Figure 5.16. Clear accumulations about $\Delta E=0, M_{E S}=m_{B}$ (where $m_{B}$ is the nominal $B$ meson mass) are observable in both the $\eta_{c} \rightarrow K_{S}^{0} K^{+} \pi^{-}$and $\eta_{c} \rightarrow K^{+} K^{-} \pi^{0}$ channels. 

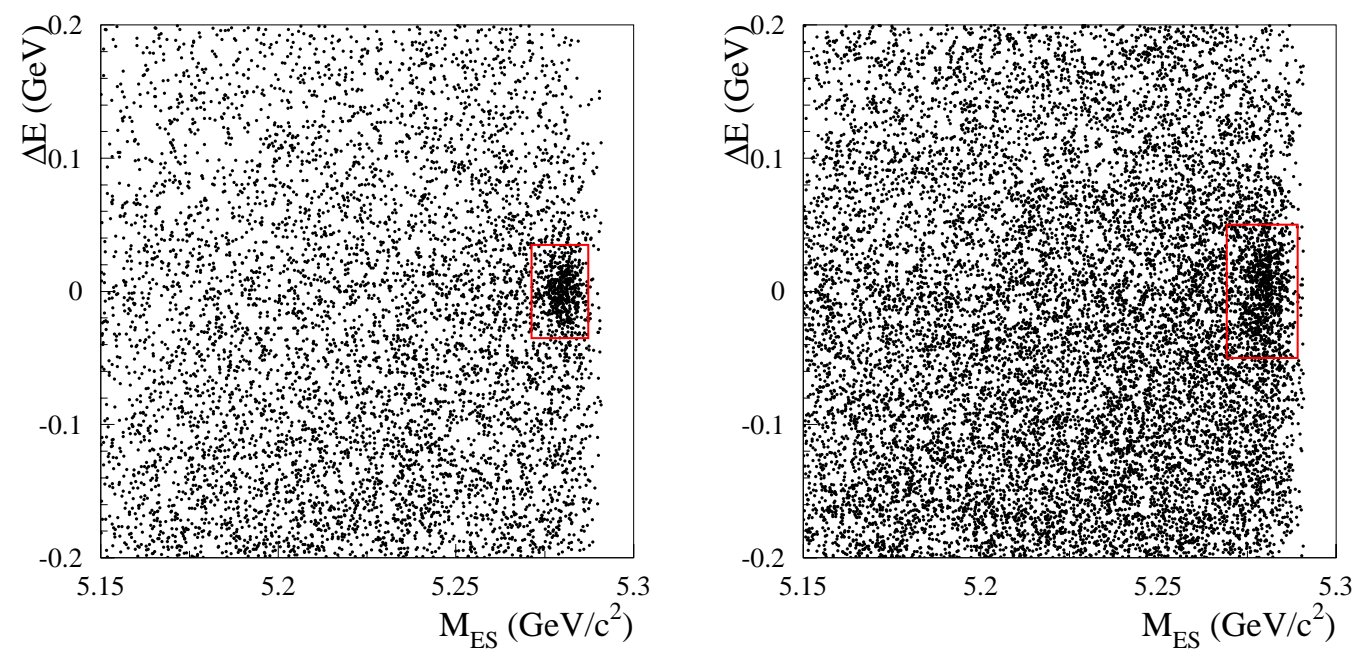

Figure 5.16: $M_{E S}$ vs. $\Delta E$ scatter plots for combined $B^{0} \rightarrow \eta_{c} K_{S}^{0}, B^{+} \rightarrow$ $\eta_{c} K^{+}$samples for $\eta_{c} \rightarrow K_{S}^{0} K^{+} \pi^{-}$(left) and $\eta_{c} \rightarrow K^{+} K^{-} \pi^{0}$ (right). The boxes denote the $M_{E S}$ and $\Delta E$ signal regions used to obtain the final yields.

The $M_{E S}$ distributions (for candidates within the $\Delta E$ signal band) are plotted for each $B \rightarrow \eta_{c} K, \eta_{c} \rightarrow K \bar{K} \pi$ channel in Figure 5.17. Fits of the sum of an ARGUS function and a Gaussian, from which the main estimation of the combinatorial background is taken, are superimposed. The $M_{E S}$ resolution (measured by the Gaussian width) in the $B^{+} \rightarrow \eta_{c} K^{+}$channel is $2.6 \pm 0.2 \mathrm{MeV} / c^{2}\left(2.9 \pm 0.3 \mathrm{MeV} / c^{2}\right)$ in the $\eta_{c} \rightarrow K_{S}^{0} K^{+} \pi^{-}\left(\eta_{c} \rightarrow K^{+} K^{-} \pi^{0}\right)$ channel. In the $B^{0} \rightarrow \eta_{c} K_{S}^{0}$ channels the number of events are fewer and the $M_{E S}$ distributions exhibit more bin-to-bin fluctuations, so the Gaussian width is fixed to $2.7 \mathrm{MeV} / c^{2}$, the value obtained from the Monte Carlo (see Section 5.3.7).

In the $B^{0} \rightarrow \eta_{c} K_{S}^{0}$ channels an excess of events is observed on the low side of the peak around $M_{E S}=m_{B}$. This is particularly noticeable in the $\eta_{c} \rightarrow K_{S}^{0} K^{+} \pi^{-}$mode which has the largest number of entries. The events do not seem to be correspond to the combinatorial background (described by the ARGUS function in $M_{E S}$ ), or the peaking background or signal (expected to be Gaussian, centred on $M_{E S}=m_{B}$ ). 

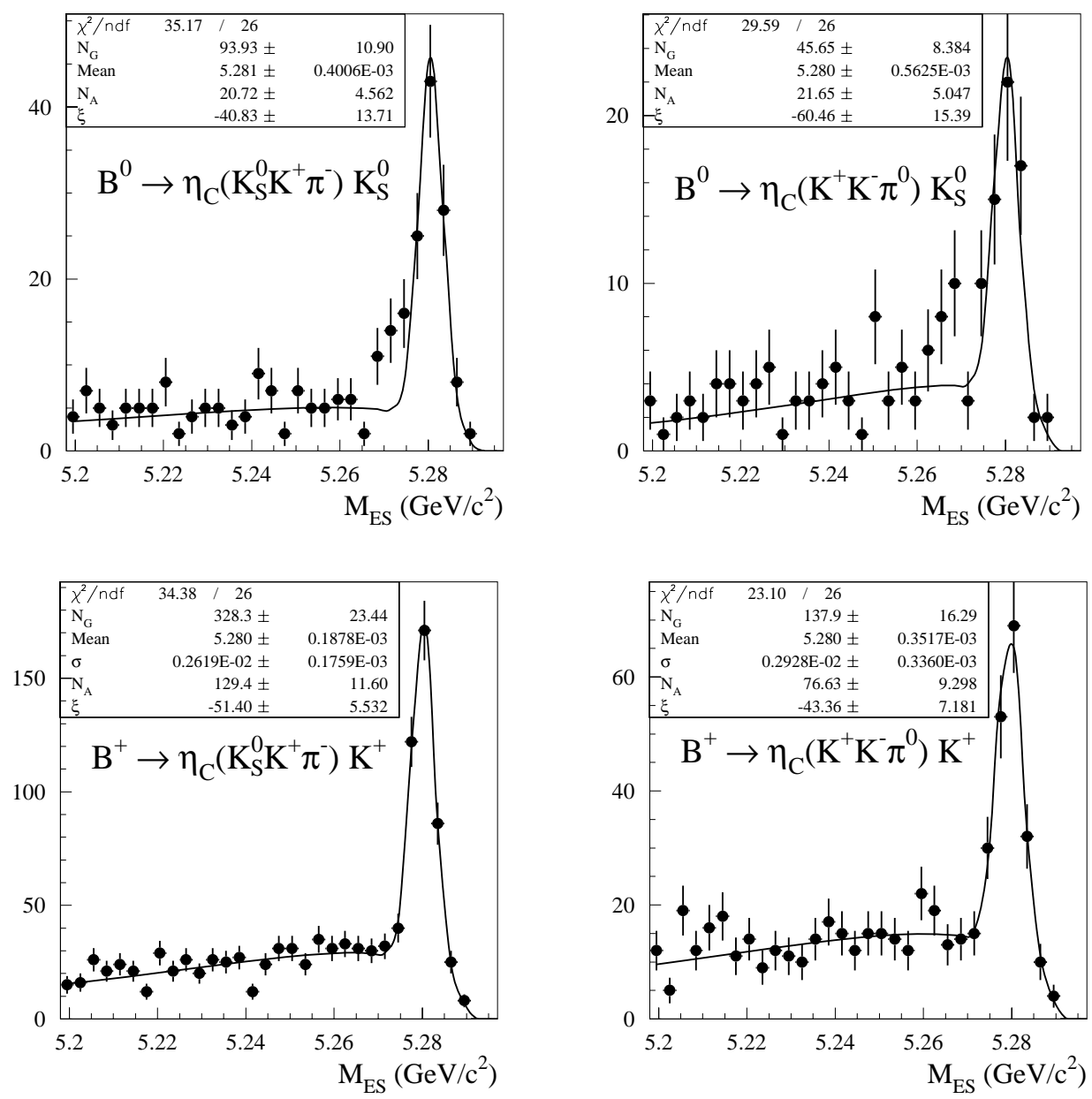

Figure 5.17: $M_{E S}$ distributions (unweighted) for $B^{0} \rightarrow \eta_{c} K_{S}^{0}$ (top) and $B^{+} \rightarrow \eta_{c} K^{+}$(bottom) channels, where $\eta_{c} \rightarrow K_{S}^{0} K^{+} \pi^{-}$(left) and $\eta_{c} \rightarrow$ $K^{+} K^{-} \pi^{0}$ (right). A fit of the sum an ARGUS function and a Gaussian is superimposed, and the fit parameters are displayed. $N_{A}$ and $N_{G}$ are number of events from the ARGUS and Gaussian integrals respectively in the $M_{E S}$ signal band.

It may be possible that the events are a statistical fluctuation of the signal or background (combinatorial or peaking) $M_{E S}$ distribution. On the other hand, they may represent some unexpected behaviour of the signal and background. Such behaviour is not observed in the SP4 signal $B^{0} \rightarrow \eta_{c} K_{S}^{0}$ Monte Carlo (see Figure 4.2 ), while detailed studies of the simulated background shaped in $M_{E S}$ have not 
been performed yet ${ }^{5}$.

This analysis proceeds on the assumption that the excess of events is a statistical fluctuation of either the signal or background $M_{E S}$ distributions and is therefore accounted for in the statistical error on the background subtracted yield. The study of systematic errors (Section 5.6.3) partially takes into account the effect of the unexpected $M_{E S}$ shape on the fits used in the background estimation.

The $\Delta E$ distributions (combined $B^{0}$ and $B^{+}$samples) are shown in Figure 5.18. For the $\eta_{c} \rightarrow K_{S}^{0} K^{+} \pi^{-}$channel, a double Gaussian fit (common mean, with linear background function) yields a $\Delta E$ mean value of approximately $-4 \mathrm{MeV}$, and an effective width of $26 \pm 5 \mathrm{MeV}$ (the width error is estimated by the error on the central Gaussian). For the $\eta_{c} \rightarrow K^{+} K^{-} \pi^{0}$ channel, a double Gaussian fit fails to converge; instead, a single Gaussian function with a linear function is used. For the $\eta_{c} \rightarrow K^{+} K^{-} \pi^{0}$ channel, a mean $\Delta E$ of approximately $-9 \mathrm{MeV}$ and a $\Delta E$ width of $39 \pm 3 \mathrm{MeV}$ is obtained. The observed offsets in the $\Delta E$ distributions are thought to be due to the pion mass hypothesis used in the momentum reconstruction of charged kaon candidates. The effect is most significant in low momentum kaon daughters of the $\eta_{c}$. In the signal Monte Carlo, using double Gaussian fits in both cases the $\Delta E$ offsets are found to be $-1 \mathrm{MeV}(-7 \mathrm{MeV})$ for the $\eta_{c} \rightarrow K_{S}^{0} K^{+} \pi^{-}\left(\eta_{c} \rightarrow K^{+} K^{-} \pi^{0}\right)$ channel, while the resolutions obtained are $43 \pm 0.91 \mathrm{MeV}(19 \pm 0.26 \mathrm{MeV})$.

The $\eta_{c}$ mass distribution for candidates within the $\Delta E, M_{E S}$ signal range is shown in Figure 5.19. A distinct peak at the $J / \psi$ mass $\left(3.1 \mathrm{GeV} / c^{2}\right)$ is visible in the $\eta_{c} \rightarrow$ $K_{S}^{0} K^{+} \pi^{-}$channel, while a hint of a $J / \psi$ signal is seen in the $\eta_{c} \rightarrow K^{+} K^{-} \pi^{0}$ channel. An estimate of the $\eta_{c}$ width was obtained by binned maximum likelihood fit to the $\eta_{c}$ mass distribution, where the fit function is sum of Breit-Wigner function (convolved with a single Gaussian), a simple Gaussian, and a zeroth order polynomial. The

\footnotetext{
${ }^{5}$ The generic $B \bar{B}$ Monte Carlo studies referred to in Section 5.4.2 use PravdaMC, which may not take into account all reconstruction effects which may contribute to the $M_{E S}$ shape.
} 

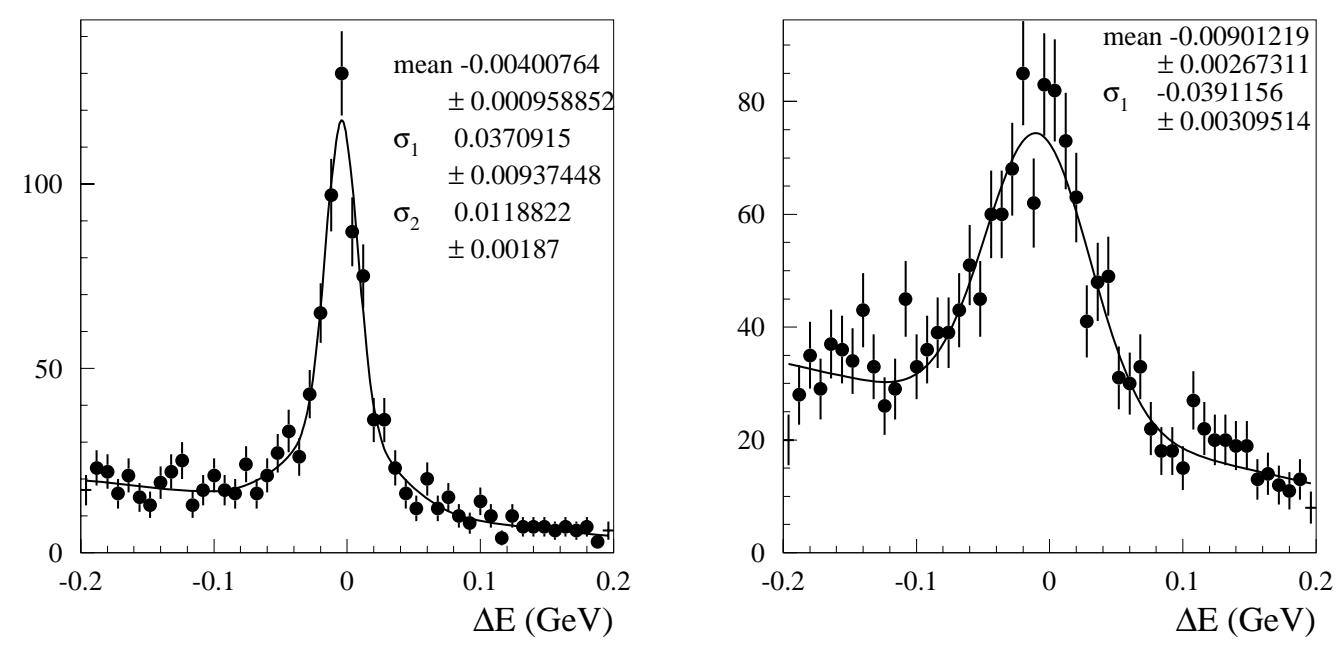

Figure 5.18: $\Delta E$ distributions for combined $B^{0} \rightarrow \eta_{c} K_{S}^{0}, B^{+} \rightarrow$ $\eta_{c} K^{+}$samples for $\eta_{c} \rightarrow K_{S}^{0} K^{+} \pi^{-}$(left) and $\eta_{c} \rightarrow K^{+} K^{-} \pi^{0}$ (right). A double Gaussian (plus first order polynomial) fit is superimposed on the $\eta_{c} \rightarrow$ $K_{S}^{0} K^{+} \pi^{-} \Delta E$ distribution; a single Gaussian (plus first order polynomial) fit is superimposed on the $\eta_{c} \rightarrow K^{+} K^{-} \pi^{0}$ distribution.

simple Gaussian mean was fixed to the nominal value of the $J / \psi$ mass, while the width was fixed to the Monte Carlo $\eta_{c} \rightarrow K \bar{K} \pi$ resolution (see Section 4.5.8). The width of the Gaussian used in the convolution was also fixed to the Monte Carlo $\eta_{c} \rightarrow K \bar{K} \pi$ resolution. The fitted Breit-Wigner width is $42.7 \pm 7.7 \mathrm{MeV} / c^{2}$ in the $\eta_{c} \rightarrow K_{S}^{0} K^{+} \pi^{-}$channel and $24.3 \pm 14.2 \mathrm{MeV} / c^{2}$ in the $\eta_{c} \rightarrow K^{+} K^{-} \pi^{0}$ channel. It must be noted that these are very preliminary measurements, whose sensitivity to systematic effects in the fits has not been studied.

Finally the $m_{K \pi}$ and $m_{\bar{K} \pi}$ distributions are plotted for each $\eta_{c} \rightarrow K \bar{K} \pi$ channel (combining the $B^{0}$ and $B^{+}$samples) in Figure 5.20. A peak at around $m_{K_{S}^{0} \pi^{-}}$, $m_{K^{+} \pi^{-}} \approx 1430 \mathrm{MeV} / c^{2}$ is visible in the $\eta_{c} \rightarrow K_{S}^{0} K^{+} \pi^{-}$channel, suggestive of the presence of $\eta_{c} \rightarrow K^{*} \bar{K}$ decays in the selection; however it must be noted that a large component of background of unknown structure is present in these plots. 

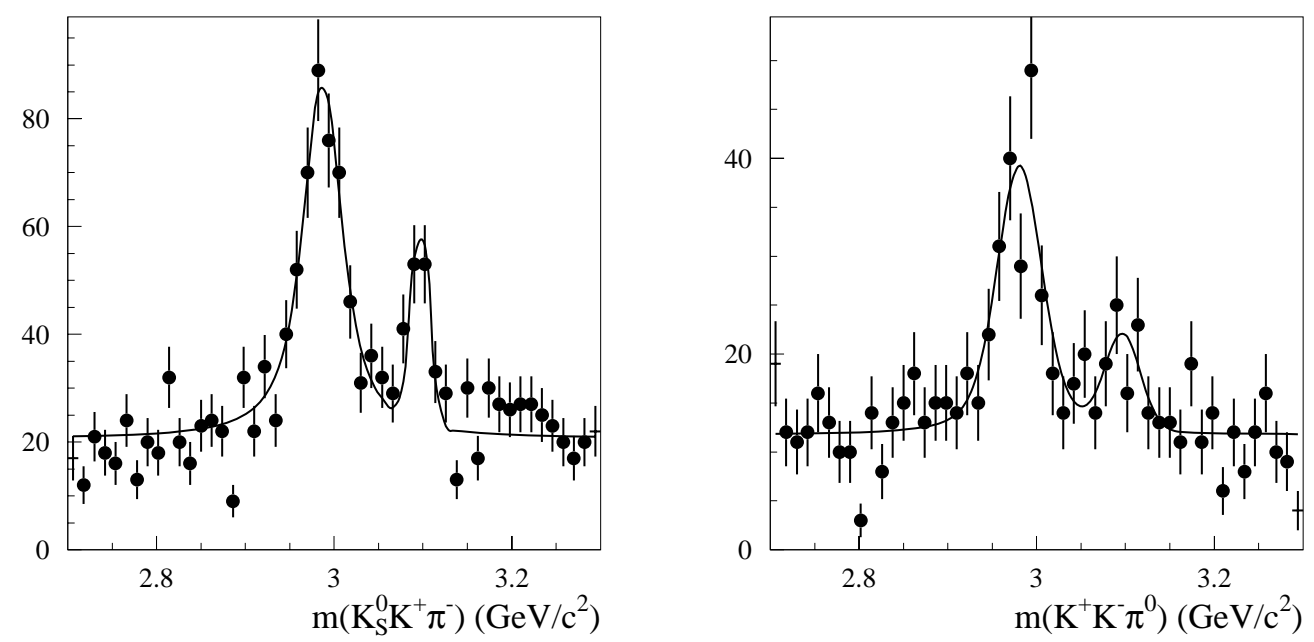

Figure 5.19: $m(K \bar{K} \pi)$ distributions for selected $B$ candidates in $\eta_{c} \rightarrow$ $K_{S}^{0} K^{+} \pi^{-}$(left), $\eta_{c} \rightarrow K^{+} K^{-} \pi^{0}$ (right) channels ( $B^{0}$ and $B^{+}$channels combined). Fits (described in text) are superimposed.
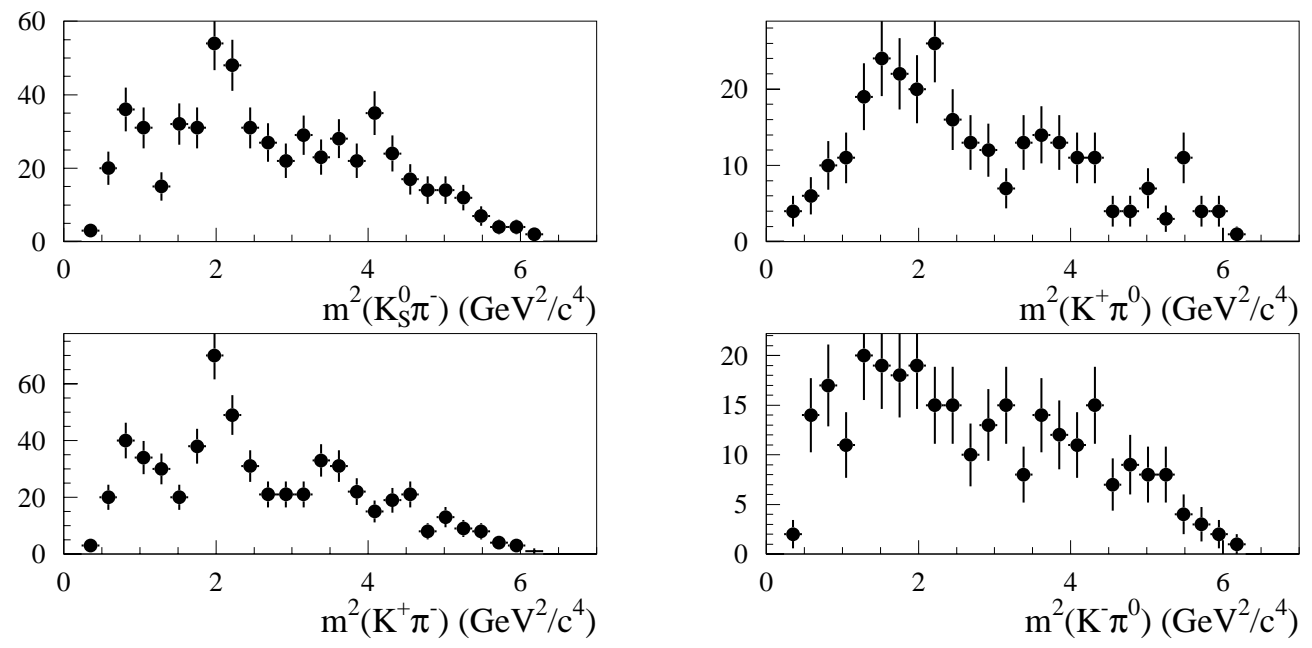

Figure 5.20: Dalitz plot projections for selected $B$ candidates in $\eta_{c} \rightarrow$ $K_{S}^{0} K^{+} \pi^{-}$(left), $\eta_{c} \rightarrow K^{+} K^{-} \pi^{0}$ (right) channels ( $B^{0}$ and $B^{+}$channels combined). 


\subsubsection{Branching Fraction Measurement Method}

The branching fractions for $B \rightarrow \eta_{c} K, \eta_{c} \rightarrow K \bar{K} \pi$ were computed with and without using efficiency weights.

The efficiency-corrected signal yields obtained without using weights can be seen in Table 5.8. At this stage, systematic errors are not included. Here for each $B \rightarrow \eta_{c} K$ channel the background-subtracted yield was computed as described in Section 5.4. To obtain the efficiency-corrected signal yield the average efficiency value was used, applying in addition the data-Monte Carlo efficiency corrections described in Section 5.3. The $\Delta E$ offsets observed in data and signal Monte Carlo are corrected for by shifting the calculated $\Delta E$ values at analysis level.

As mentioned above, the Gaussian width was fixed to $2.7 \mathrm{MeV} / c^{2}$ when performing the fits to the $M_{E S}$ distributions in the $\Delta E$ and $\mathrm{m}\left(\eta_{c}\right)$ signal bands for the $B^{+} \rightarrow$ $\eta_{c} K^{+}$channels, to extract the combinatorial background. When extracting the peaking background, using the fit to the $M_{E S}$ distribution in the $\mathrm{m}\left(\eta_{c}\right)$ sidebands, the Gaussian width was fixed to $2.7 \mathrm{MeV} / c^{2}$ in all channels; these fits are shown in Figure 5.21. The fit is poorest in the $B^{0} \rightarrow \eta_{c} K_{S}^{0}, \eta_{c} \rightarrow K^{+} K^{-} \pi^{0}$ channel, where the number of events are the fewest and there are large fluctuations in the bin contents.

The efficiency-corrected signal yields for the weighted data samples can be seen in Table 5.9. The weights for each candidate were determined by the procedure described in Section 5.2, using both the efficiency parametrizations based on the $K_{A} \pi$ and $K_{B} \pi$ subsystems from $\eta_{c} \rightarrow K_{A} K_{B} \pi$. Legendre polynomials up to 3rd order were used to parametrize the efficiency variation in the helicity cosine. DataMonte Carlo efficiency corrections are used to correct the yield after background subtraction. Again, only statistical errors are shown.

The efficiency-corrected yields are observed to be quite similar using either the 

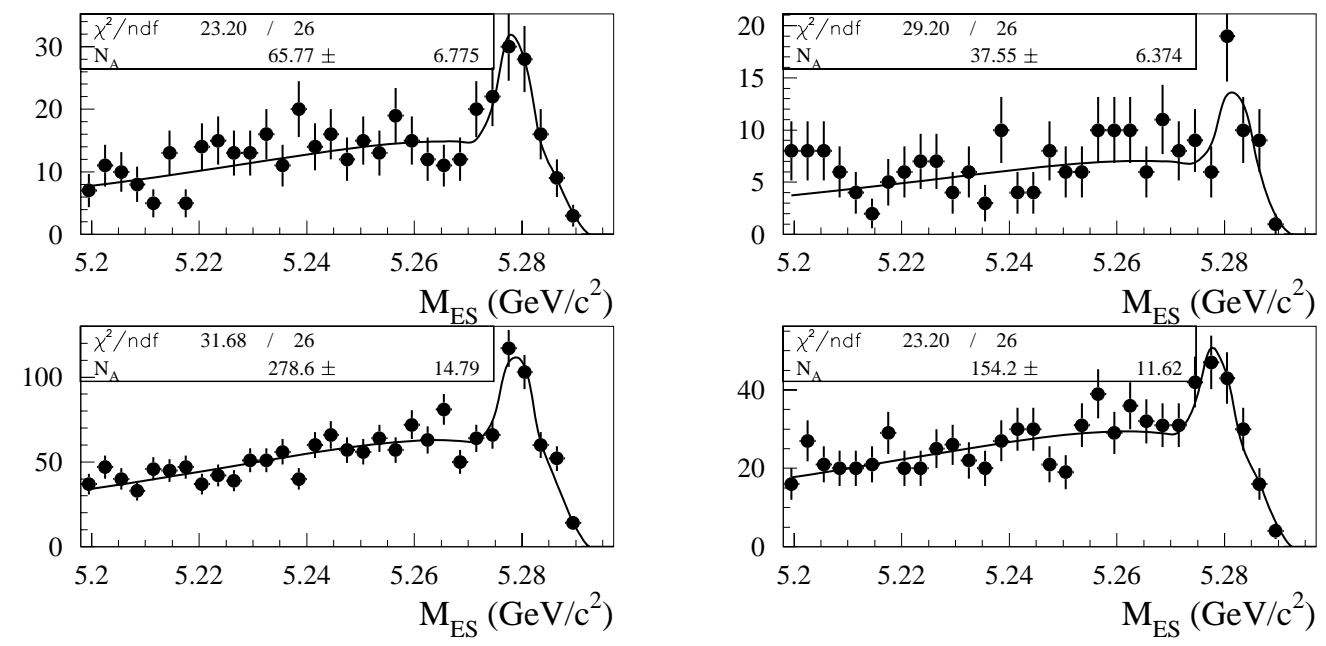

Figure 5.21: $M_{E S}$ distributions (unweighted) plotted for candidates occupying the $\Delta E$ signal band and $m\left(\eta_{c}\right)$ side bands, for $\eta_{c} \rightarrow K_{S}^{0} K^{+} \pi^{-}$(left) and $\eta_{c} \rightarrow K^{+} K^{-} \pi^{0}$ (right); $B^{0} \rightarrow \eta_{c} K_{S}^{0}$ (top), $B^{+} \rightarrow \eta_{c} K^{+}$(bottom). Fits (sum of ARGUS and Gaussian functions, Gaussian width $=2.7 \mathrm{MeV} / \mathrm{c}^{2}$ ) are superimposed. The excess of events in the $M_{E S}$ signal region, extrapolated to the $m\left(\eta_{c}\right)$ signal region estimates the peaking background.

weighted or unweighted data samples. Some difference is seen in the $\eta_{c} \rightarrow K^{+} K^{-} \pi^{0}$ channels using different efficiency parametrizations.

The combinatorial background estimated by the methods A and B described in Section 5.4.4 are in quite good agreement in most cases.

Preliminary branching fractions may be obtained from these efficiency-corrected signal yields, and are shown in Table 5.10. From these results, it can be seen that the ratio of branching fraction satisfy isospin symmetry (see Section 1.6.3) since

$$
\begin{aligned}
& \frac{\mathcal{B}\left(B^{0} \rightarrow \eta_{c} K^{0}\right) \times \mathcal{B}\left(\eta_{c} \rightarrow K^{0} K^{+} \pi^{-}\right)}{\mathcal{B}\left(B^{0} \rightarrow \eta_{c} K^{0}\right) \times \mathcal{B}\left(\eta_{c} \rightarrow K^{+} K^{-} \pi^{0}\right)}=0.28 \pm 0.09, \\
& \frac{\mathcal{B}\left(B^{+} \rightarrow \eta_{c} K^{+}\right) \times \mathcal{B}\left(\eta_{c} \rightarrow K^{0} K^{+} \pi^{-}\right)}{\mathcal{B}\left(B^{+} \rightarrow \eta_{c} K^{+}\right) \times \mathcal{B}\left(\eta_{c} \rightarrow K^{+} K^{-} \pi^{0}\right)}=0.26 \pm 0.06,
\end{aligned}
$$

(where only the statistical error is given). 
Table 5.8: Efficiency-corrected yields for $B \rightarrow \eta_{c} K, \eta_{c} \rightarrow K \bar{K} \pi$ using unweighted data sample. (A) and (B) refer to the methods described in Section 5.4.4. The efficiency-corrected yield includes data-Monte Carlo efficiency corrections.

\begin{tabular}{c|c|c|c|c}
\hline \multirow{2}{*}{$\eta_{c}$ Decay Channel } & \multicolumn{2}{|c|}{$K_{S}^{0} K^{+} \pi^{-}$} & \multicolumn{2}{c}{$K^{+} K^{-} \pi^{0}$} \\
\cline { 2 - 5 } & $B^{0}$ & $B^{+}$ & $B^{0}$ & $B^{+}$ \\
\hline \hline Total yield & $125 \pm 11.2$ & $460 \pm 21.4$ & $74 \pm 8.6$ & $216 \pm 14.7$ \\
Comb. background (A) & $20.7 \pm 4.6$ & $129.4 \pm 11.6$ & $21.7 \pm 5.1$ & $76.6 \pm 9.3$ \\
Comb. background (B) & $30.7 \pm 3.2$ & $130.1 \pm 6.9$ & $17.4 \pm 2.7$ & $71.9 \pm 5.4$ \\
Peaking background & $21.1 \pm 5.9$ & $67.8 \pm 11.8$ & $12.5 \pm 4.6$ & $33.5 \pm 8.8$ \\
Background-subtracted yield (A) & $83.2 \pm 13.4$ & $262.9 \pm 27.1$ & $39.8 \pm 11.0$ & $105.9 \pm 19.5$ \\
Efficiency & $0.181 \pm 0.002$ & $0.198 \pm 0.002$ & $0.112 \pm 0.002$ & $0.111 \pm 0.002$ \\
Efficiency-corrected yield (A) & $441.7 \pm 71.2$ & $1314.3 \pm 135.6$ & $361.5 \pm 100.2$ & $995.6 \pm 183.8$ \\
\hline
\end{tabular}

Table 5.9: Efficiency corrected yields with weights. All results use weights from the $K_{S}^{0} \pi\left(K^{+} \pi^{0}\right)$ efficiency parametrization for the $\eta_{c} \rightarrow K_{S}^{0} K^{+} \pi^{-}\left(\eta_{c} \rightarrow K^{+} K^{-} \pi^{0}\right)$ mode, except those in parenthesis, which use the $K \pi\left(K^{-} \pi^{0}\right)$ weights.(A) and (B) refer to the methods described in Section 5.4.4. The background-subtracted yield includes data-Monte Carlo efficiency corrections.

\begin{tabular}{c|c|c|c|c}
\hline \multirow{2}{*}{$\eta_{c}$ Decay Channel } & \multicolumn{2}{|c|}{$K_{S}^{0} K^{+} \pi^{-}$} & \multicolumn{2}{c}{$K^{+} K^{-} \pi^{0}$} \\
\cline { 2 - 5 } & $B^{0}$ & $B^{+}$ & $B^{0}$ & $B^{+}$ \\
\hline \hline Total yield & $692.0 \pm 63.7$ & $2310.4 \pm 108.7$ & $686.7 \pm 82.2$ & $2008.3 \pm 139.7$ \\
Comb. background (A) & $118.1 \pm 26.9$ & $670.0 \pm 60.1$ & $180.7 \pm 42.9$ & $726.9 \pm 87.5$ \\
Comb. background (B) & $167.1 \pm 17.2$ & $668.9 \pm 35.7$ & $160.3 \pm 28.4$ & $638.9 \pm 48.8$ \\
Peaking background & $124.7 \pm 33.4$ & $347.7 \pm 61.3$ & $112.8 \pm 45.0$ & $356.9 \pm 83.6$ \\
Background-subtracted & $431.7 \pm 73.8$ & $1279.6 \pm 137.1$ & $400.0 \pm 104.9$ & $964.8 \pm 192.9$ \\
yield (A) & $(413.9 \pm 72.2)$ & $(1291.3 \pm 138.5)$ & $(360.1 \pm 104.0)$ & $(957.9 \pm 183.6)$ \\
\hline
\end{tabular}

The difference in the preliminary branching fractions, using the weighted and unweighted data samples is at the $\sim 6 \%$ level (except for the $B^{0} \rightarrow \eta_{c} K_{S}^{0}, \eta_{c} \rightarrow$ $K^{+} K^{-} \pi^{0}$ channel using the $K^{+} \pi^{0}$ weights). Taking into account the systematic 
Table 5.10: Measured branching fractions ('B.F.') for $B \rightarrow \eta_{c} K, \eta_{c} \rightarrow K \bar{K} \pi$, using efficiency-weighted and unweighted data samples. The $K_{A} \pi$ and $K_{B} \pi$ subsystems, used to compute the efficiency weights, are described in the text.

\begin{tabular}{c|c|c|c|c}
\hline \multirow{2}{*}{ B.F. $\left(\times 10^{-5}\right)$} & \multicolumn{2}{|c|}{$\mathcal{B}\left(\eta_{c} \rightarrow K^{0} K^{+} \pi^{-}\right) \times$} & \multicolumn{2}{c}{$\mathcal{B}\left(\eta_{c} \rightarrow K^{+} K^{-} \pi^{0}\right) \times$} \\
\cline { 2 - 5 } & $\mathcal{B}\left(B^{0} \rightarrow \eta_{c} K^{0}\right)$ & $\mathcal{B}\left(B^{+} \rightarrow \eta_{c} K^{+}\right)$ & $\mathcal{B}\left(B^{0} \rightarrow \eta_{c} K^{0}\right)$ & $\mathcal{B}\left(B^{+} \rightarrow \eta_{c} K^{+}\right)$ \\
\hline \hline Unweighted & $4.36 \pm 0.70$ & $4.45 \pm 0.46$ & $1.24 \pm 0.34$ & $1.17 \pm 0.22$ \\
Weighted $\left(K_{A} \pi\right)$ & $4.26 \pm 0.73$ & $4.33 \pm 0.46$ & $1.37 \pm 0.36$ & $1.13 \pm 0.23$ \\
Weighted $\left(K_{B} \pi\right)$ & $4.08 \pm 0.71$ & $4.37 \pm 0.47$ & $1.23 \pm 0.36$ & $1.13 \pm 0.22$ \\
\hline
\end{tabular}

errors involved in the weighting procedure and background subtraction, it appears the branching fractions are not very sensitive to selection efficiency variation in the $\eta_{c}$ decay phase space (using the present set of selections). It therefore seems reasonable to measure the branching fractions using the unweighted samples, and assign a systematic error to account for this sensitivity.

A full treatment of possible systematic errors is necessary to complete the branching fraction measurement; this is detailed in the next section.

\subsubsection{Systematic Errors}

The systematic errors arise from several sources to affect the ingredients of the branching fraction measurement. These are listed below.

\section{Efficiency Variation in $\eta_{c}$ Decay Phase Space}

The central value of each $B \rightarrow \eta_{c} K, \eta_{c} \rightarrow K \bar{K} \pi$ branching fraction is taken to be that obtained using the average selection efficiency (unweighted samples) for each channel. 
Although some of the change in the measured branching fraction using a weighted data sample may be due to the presence of resonant $\eta_{c}$ decays in the data, it is likely that much of the change is due to systematic errors in the efficiency parametrization itself and possible biases in the background distributions which are weighted before subtraction. Without a more sophisticated analysis to disentangle these effects, a conservative approach is to simple apply the change in the branching fraction as a systematic error.

Thus, the systematic error made in using the unweighted samples is estimated by the difference seen in the value of the branching fraction measured using the weighted data samples. Since the weighted data samples use two parametrizations, the largest difference is used as the systematic error. This systematic error is at the level of $\lesssim 6 \%$, except in the $B^{0} \rightarrow \eta_{c} K_{S}^{0}, \eta_{c} \rightarrow K^{+} K^{-} \pi^{0}$ channel where it is $\approx 11 \%$.

There is also a statistical error on the average selection efficiency, due to the finite size of the signal Monte Carlo samples. However the samples are sufficiently large that this error is almost negligible ( $<0.2 \%$ for each decay mode).

\section{Monte Carlo Discrepancies}

There are several errors on the Monte Carlo efficiency measurement arising from inaccurate simulation of data. The methods to correct for these discrepancies and the errors involved were described in Section 5.3. A detailed breakdown is given in Table 5.4.

The accuracy of the simulated $\Delta E$ and $M_{E S}$ distributions was examined by direct comparison of signal Monte Carlo and data after all selections were applied. The observed offsets of the $\Delta E$ mean were corrected by shifting the $\Delta E$ distribution in data and Monte Carlo before computing final efficiencies and yields (see Section 5.6.1). The $\Delta E$ resolution is more difficult to determine in data than in Monte 
Carlo due to the lower number of events and the presence of background, which may accumulate in the region of the signal peak (due to the requirement that the candidate with the smallest value of $\Delta E$ is retained in the case of multiple candidates). Figure 5.22 shows a normalised comparison of the background-subtracted data and Monte Carlo $\Delta E$ distributions. Fits to the $\Delta E$ distributions (which assume the background is linear in $\Delta E$ ) reveal a larger $\Delta E$ width in the data $\eta_{c} \rightarrow K_{S}^{0} K^{+} \pi^{-}$ channels than is observed in Monte Carlo (see Section 5.6.1), primarily due to the presence of substantially larger $\Delta E$ tails. The fractional integral of the signal component (double Gaussian) of the $\eta_{c} \rightarrow K_{S}^{0} K^{+} \pi^{-} \Delta E$ fit function within the signal range $(|\Delta E|<0.035)$ is $6.9 \%$ lower in the data than in Monte Carlo. Thus the Monte Carlo efficiency is rescaled by a correction factor of 0.931 , and the associated systematic error on the efficiency is taken to be half the value of the correction $(3.45 \%)$.

In the $\eta_{c} \rightarrow K^{+} K^{-} \pi^{0}$ channel the $\Delta E$ comparison is more difficult due to the lower event yield in the data, which meant a double Gaussian fit could not be used. Rather than make the comparison using single a Gaussian fit which is clearly inadequate to describe the Monte Carlo, the efficiency correction and systematic error from the $\eta_{c} \rightarrow K_{S}^{0} K^{+} \pi^{-}$channel is used. This is a rather arbitrary method of assigning the systematic error but is preferable to neglecting the effect completely.

In the $B^{+} \rightarrow \eta_{c} K^{+}$channels, the measured $M_{E S}$ resolution (see Section 5.6.1) is consistent with that seen in Monte Carlo (see Section 5.3.7). Since the $M_{E S}$ resolution is dominated by the beam energy spread rather than experimental resolution, a difference would not be expected in the $B^{0} \rightarrow \eta_{c} K_{S}^{0}$ channels. Thus no systematic error is assigned for the use of the $M_{E S}$ selection variable in this analysis.

\section{Background Estimation}

The combinatorial and peaking background estimations are affected by the system- 

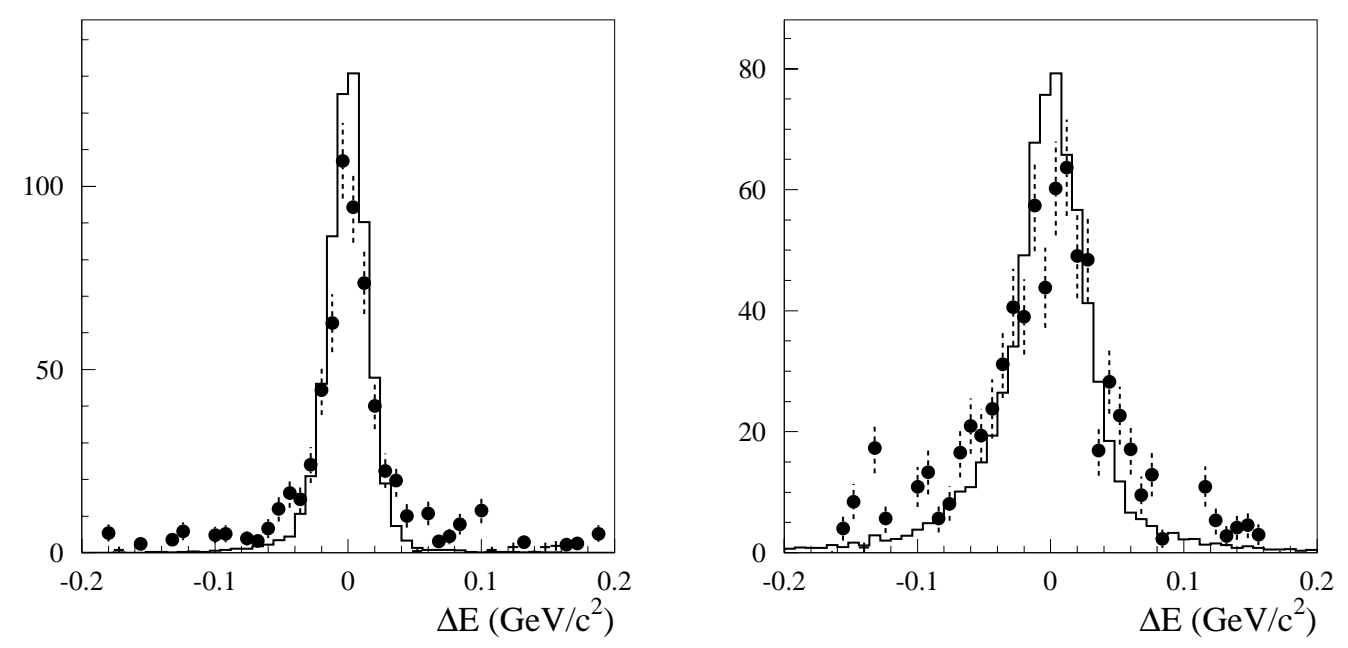

Figure 5.22: $\Delta E$ distributions in data (points) and Monte Carlo (line) for $\eta_{c} \rightarrow K_{S}^{0} K^{+} \pi^{-}$(left) and $\eta_{c} \rightarrow K^{+} K^{-} \pi^{0}$ (right). Both $B^{0} \rightarrow \eta_{c} K_{S}^{0}$ and $B \rightarrow$ $\eta_{c} K$ channels are combined. A bin-by-bin background subtraction is carried out in the data. (In the Monte Carlo, the proportions in the combination is chosen to match the appropriate proportion of signal yields in data).

atic errors involved in the fits used, which may result from a poor description of the data by the fitting function (the sum of an ARGUS function and a Gaussian).

It may be expected that the systematic errors involved in the fits to the $M_{E S}$ distributions will approximately cancel in the background subtraction (5.16), if the systematic effects in the fits are fully correlated in the $\mathrm{m}\left(\eta_{c}\right)$ signal band and sidebands. In that case the total estimated background would be insensitive to the systematic errors.

The systematic error on the ARGUS description may be probed by extending the range of the fit to $5.15 \mathrm{GeV} / c^{2}<M_{E S}<5.3 \mathrm{GeV} / c^{2}$. Figure 5.23 shows the fits to the $M_{E S}$ distributions in the $B^{+} \rightarrow \eta_{c} K^{+}, \eta_{c} \rightarrow K_{S}^{0} K^{+} \pi^{-}$channel. The integrals of the ARGUS functions in the $M_{E S}$ signal region are observed to decrease with 
respect to those in Section 5.6.2, by approximately 15\%. However, the systematic effect does indeed cancel to some extent in this channel and the total background changes by $\sim 5 \%$ compared to that estimated in Section 5.6.2.
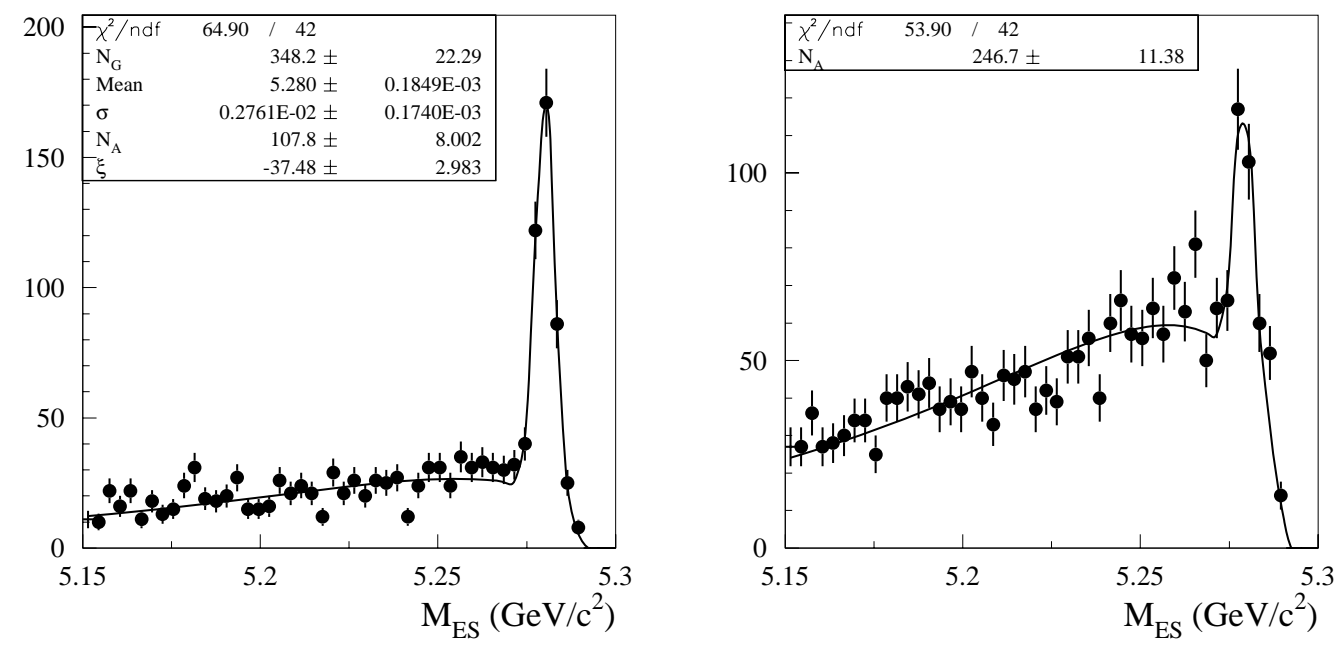

Figure 5.23: $M_{E S}$ distributions in $B^{+} \rightarrow \eta_{c} K^{+}, \eta_{c} \rightarrow K_{S}^{0} K^{+} \pi^{-}$(unweighted) plotted for candidates occupying the $\Delta E$ signal band and $m\left(\eta_{c}\right)$ signal band (left) and $m\left(\eta_{c}\right)$ side bands (right). Fits (sum of ARGUS and Gaussian functions) are superimposed.

The effect of varying the fit range on the total estimated background for all $B \rightarrow \eta_{c} K$ channels in shown Table 5.11. The fractional change in background-subtracted yield from varying the fit range is taken as a systematic error on the branching fraction.

In the $B^{0} \rightarrow \eta_{c} K_{S}^{0}$ channels, the Gaussian width was a fixed parameter in the fit to the $M_{E S}$ distributions in both the $\mathrm{m}\left(\eta_{c}\right)$ sidebands and signal band. The effect of allowing the parameter to float on the total background is given in Table 5.12. In the $\eta_{c} \rightarrow K^{+} K^{-} \pi^{0}$ channel the fit to the $M_{E S}$ distribution in the $\mathrm{m}\left(\eta_{c}\right)$ sidebands fails to converge unless the Gaussian width is fixed $3.1 \mathrm{MeV} / c^{2}$ (the value obtained from the fit in the $\Delta E$ signal band). The small resulting difference to the backgroundsubtracted yield is added in quadrature to the systematic error on the branching 
Table 5.11: Total background estimated used the fit ranges (1) $5.2 \mathrm{GeV} / \mathrm{c}^{2}<M_{E S}<$ $5.3 \mathrm{GeV} / \mathrm{c}^{2}$ (2) $5.15 \mathrm{GeV} / \mathrm{c}^{2}<M_{E S}<5.3 \mathrm{GeV} / \mathrm{c}^{2}$.

\begin{tabular}{c|c|c|c|c}
\hline \multirow{2}{*}{$\eta_{c}$ Decay Channel } & \multicolumn{2}{|c|}{$K_{S}^{0} K^{+} \pi^{-}$} & \multicolumn{2}{c}{$K^{+} K^{-} \pi^{0}$} \\
\cline { 2 - 5 } & $B^{0}$ & $B^{+}$ & $B^{0}$ & $B^{+}$ \\
\hline \hline Total background (1) & $41.8 \pm 7.4$ & $197.1 \pm 16.6$ & $34.2 \pm 6.9$ & $110.1 \pm 12.9$ \\
Total background (2) & $47.6 \pm 6.3$ & $190.5 \pm 13.6$ & $32.5 \pm 5.4$ & $112.1 \pm 10.9$ \\
\hline
\end{tabular}

fraction.

Table 5.12: Total background estimated (1) Gaussian width fixed to $2.7 \mathrm{MeV} / \mathrm{c}^{2}$ (2) Gaussian width as free parameter.

\begin{tabular}{c|c|c}
\hline$\eta_{c}$ Decay Channel & $K_{S}^{0} K^{+} \pi^{-}\left(B^{0}\right)$ & $K^{+} K^{-} \pi^{0}\left(B^{0}\right)$ \\
\hline Total background (1) & $41.8 \pm 7.4$ & $34.2 \pm 6.9$ \\
Total background (2) & $38.5 \pm 7.4$ & $34.0 \pm 7.3$ \\
\hline
\end{tabular}

\section{B Counting}

The error on the estimated number of $B \bar{B}$ pairs in the data sample is $1.1 \%$ (see Section 5.5).

\section{Summary}

The systematic errors discussed above are summarised in Table 5.13. The errors due to data-Monte Carlo discrepancies from the $\Delta E$ selection and the other sources described in Section 5.3 are summed in quadrature. 
Table 5.13: Summary of Branching Fraction Systematic Errors. All numbers are relative percentage. The systematic errors associated with the $\Delta E$ selection (see text) are included in the Monte Carlo discrepancies.

\begin{tabular}{c|c|c|c|c}
\hline \multirow{2}{*}{$\eta_{c}$ Decay Channel } & \multicolumn{2}{|c|}{$K_{S}^{0} K^{+} \pi^{-}$} & \multicolumn{2}{|c}{$K^{+} K^{-} \pi^{0}$} \\
\cline { 2 - 5 } & $B^{0}$ & $B^{+}$ & $B^{0}$ & $B^{+}$ \\
\hline \hline$\eta_{c}$ phase space efficiency (\%) & 6.3 & 2.6 & 10.7 & 3.8 \\
Data-Monte Carlo discrepancies (\%) & 11.0 & 8.8 & 9.8 & 9.6 \\
Background subtraction (\%) & 8.0 & 2.5 & 4.3 & 1.9 \\
$B$ counting (\%) & 1.1 & 1.1 & 1.1 & 1.1 \\
Total (\%) & 15.2 & 9.6 & 15.1 & 10.5 \\
\hline
\end{tabular}

\subsubsection{Branching Fraction Results}

The branching fraction results for $\mathcal{B}\left(B \rightarrow \eta_{c} K\right) \times \mathcal{B}\left(\eta_{c} \rightarrow K \bar{K} \pi\right)$ are given by

$$
\begin{aligned}
\mathcal{B}\left(B^{0} \rightarrow \eta_{c} K^{0}\right) \times \mathcal{B}\left(\eta_{c} \rightarrow K^{0} K^{+} \pi^{-}\right) & =(4.36 \pm 0.70 \pm 0.66) \times 10^{-5} \\
\mathcal{B}\left(B^{+} \rightarrow \eta_{c} K^{+}\right) \times \mathcal{B}\left(\eta_{c} \rightarrow K^{0} K^{+} \pi^{-}\right) & =(4.45 \pm 0.46 \pm 0.42) \times 10^{-5} \\
\mathcal{B}\left(B^{0} \rightarrow \eta_{c} K^{0}\right) \times \mathcal{B}\left(\eta_{c} \rightarrow K^{+} K^{-} \pi^{0}\right) & =(1.24 \pm 0.34 \pm 0.19) \times 10^{-5} \\
\mathcal{B}\left(B^{+} \rightarrow \eta_{c} K^{+}\right) \times \mathcal{B}\left(\eta_{c} \rightarrow K^{+} K^{-} \pi^{0}\right) & =(1.17 \pm 0.22 \pm 0.12) \times 10^{-5}
\end{aligned}
$$

where the first result is statistical and the second systematic.

Since these branching fractions are consistent with isospin symmetry of the $\eta_{c} \rightarrow$ $K^{0} K^{+} \pi^{-}, \eta_{c} \rightarrow K \bar{K} \pi$ channels, the results can be combined to obtain the branching fractions $\mathcal{B}\left(B^{0} \rightarrow \eta_{c} K_{S}^{0}\right), \mathcal{B}\left(B \rightarrow \eta_{c} K\right)$. Since a large component of the systematic error is correlated between the channels only the statistical error is used in the weighted average. The largest systematic error when combining two results is used as the final fractional systematic error. 
This gives

$$
\begin{aligned}
\mathcal{B}\left(B^{0} \rightarrow \eta_{c} K^{0}\right) & =(1.24 \pm 0.17 \pm 0.19 \pm 0.38) \times 10^{-3} \\
\mathcal{B}\left(B^{+} \rightarrow \eta_{c} K^{+}\right) & =(1.23 \pm 0.11 \pm 0.12 \pm 0.38) \times 10^{-3},
\end{aligned}
$$

where the first error is statistical, the second systematic, and the third is the error on the $\eta_{c} \rightarrow K \bar{K} \pi$ branching fraction [5].

\subsection{Conclusion}

The branching fractions $\mathcal{B}\left(B \rightarrow \eta_{c} K\right)$ measured above represent an improvement in accuracy compared to the previous BABAR measurements (the Run 1 analysis) which were [37]

$$
\begin{aligned}
\mathcal{B}\left(B^{0} \rightarrow \eta_{c} K^{0}\right) & =(1.06 \pm 0.28 \pm 0.11 \pm 0.33) \times 10^{-3} \\
\mathcal{B}\left(B^{+} \rightarrow \eta_{c} K^{+}\right) & =(1.50 \pm 0.19 \pm 0.15 \pm 0.46) \times 10^{-3} .
\end{aligned}
$$

These results obtained using a very similar analysis in most respects, except that the possible impact of $\eta_{c} \rightarrow K^{*} \bar{K} \rightarrow K_{S}^{0} K^{+} \pi^{-}$decays was ignored. While the statistical error has certainly improved the systematic (second) error has increased, due mainly to the fact that the effect of $\eta_{c} \rightarrow K^{*} \bar{K} \rightarrow K_{S}^{0} K^{+} \pi^{-}$contributions on the branching fraction are considered in this analysis. In addition, the smaller yields meant that systematic errors on the background estimation could not be evaluated as thoroughly in the Run 1 analysis. The method of combining measurements in the $\eta_{c} \rightarrow K_{S}^{0} K^{+} \pi^{-}$and $\eta_{c} \rightarrow K^{+} K^{-} \pi^{0}$ channels presented here leads to a conservative estimate of the systematic error.

Another preliminary measurement of $\mathcal{B}\left(B \rightarrow \eta_{c} K\right)$ was performed using the same data sample in a parallel analysis [42], using the decay modes $\eta_{c} \rightarrow K^{+} K^{-} \pi^{0}$ and $\eta_{c} \rightarrow K_{S}^{0} K^{+} \pi^{-}$, but different selections in the $\eta_{c} \rightarrow K^{+} K^{-} \pi^{0}$ modes. The method 
used a two-dimensional unbinned maximum likelihood fit in $M_{E S}$ and $m\left(\eta_{c}\right)$ to extract signal yields and efficiencies. The $M_{E S}$ distribution was modelled using the sum of an ARGUS function and a Gaussian. The $m\left(\eta_{c}\right)$ distribution was modelled with the sum of Breit-Wigner (convolved with a double Gaussian resolution function), a double Gaussian (where the mean was fixed to the nominal $J / \psi$ mass), and a linear background function. The fit was performed over the range $5.2 \mathrm{GeV} / c^{2}<$ $M_{E S}<5.3 \mathrm{GeV} / c^{2}, 2.8 \mathrm{GeV} / c^{2}<m\left(\eta_{c}\right)<3.2 \mathrm{GeV} / c^{2}$. The results were

$$
\begin{aligned}
\mathcal{B}\left(B^{0} \rightarrow \eta_{c} K^{0}\right) & =(0.99 \pm 0.13 \pm 0.07 \pm 0.31) \times 10^{-3} \\
\mathcal{B}\left(B^{+} \rightarrow \eta_{c} K^{+}\right) & =(1.15 \pm 0.09 \pm 0.07 \pm 0.36) \times 10^{-3} .
\end{aligned}
$$

which show some difference in the central values compared to the results from this analysis, which is surprising considering almost the same events are used in both analyses.

In [42], no correction to the efficiency was made to account for differences in the data and Monte Carlo $\Delta E$ distributions. In this analysis, a correction of $6.9 \%$ is applied; if it is omitted the central values of $\mathcal{B}\left(B^{0} \rightarrow \eta_{c} K^{0}\right)$ and $\mathcal{B}\left(B^{+} \rightarrow \eta_{c} K^{+}\right)$ change to 1.16 and 1.15 respectively. This brings the $\mathcal{B}\left(B^{+} \rightarrow \eta_{c} K^{+}\right)$measurement into close agreement between both analyses; however there is still some discrepancy in the $\mathcal{B}\left(B^{0} \rightarrow \eta_{c} K^{0}\right)$ measurement. The reason for this discrepancy is not obvious. In this analysis, potential mistakes in the selection procedure were checked for by a comparison with a separately written selection algorithm ${ }^{6}$. The event yields after identical selection application in the two cases were identical. The fitting methods in [42] were validated by multiple $t o y^{7}$ Monte Carlo pseudo-experiments and shown to be unbiased. The method used here was tested on a non-toy (PravdaMc) Monte Carlo sample (see Section 5.4.4), and obtained the expected result; however this test does not show that the method is unbiased.

\footnotetext{
${ }^{6}$ Written by W. Kozanecki (Charmonium Working Group).

${ }^{7}$ Toy Monte Carlo is the simulation of expected distributions according to the fit model.
} 
Comparisons of the individual ingredients of the branching fraction (efficiencies and yields) in the two analyses is not straightforward since the yields and efficiencies in [42] are obtained from a different range of $M_{E S}$ and $m\left(\eta_{c}\right)$. In general the efficiencies and yields are higher (as expected) in [42], except for the $B^{0} \rightarrow \eta_{c} K_{S}^{0}$, $\eta_{c} \rightarrow K_{S}^{0} K^{+} \pi^{-}$channel, where the signal yield is higher in this analysis, feeding into a larger measured value for the $B^{0} \rightarrow \eta_{c} K^{0}$ branching fraction.

The $\mathcal{B}\left(B^{0} \rightarrow \eta_{c} K^{0}\right)$ measurement is dominated by the $\mathcal{B}\left(B^{0} \rightarrow \eta_{c} K^{0}\right) \times \mathcal{B}\left(\eta_{c} \rightarrow\right.$ $K^{0} K^{+} \pi^{-}$) measurement, and the systematic error on the background subtraction in this channel is rather large (8\%) in the method used in this analysis. The difference between the combinatorial background estimated from methods $\mathrm{A}$ and $\mathrm{B}$ is also large in this analysis (although this was not added to the systematic error) with the smaller background value used in the branching fraction measurement. Thus different performances of the background modelling between the two methods may account for some of the remaining discrepancy in the central values of the two $\mathcal{B}\left(B^{0} \rightarrow \eta_{c} K^{0}\right)$ measurements. In addition, uncorrelated statistical fluctuations may be present in the $B^{0} \rightarrow \eta_{c} K_{S}^{0}, \eta_{c} \rightarrow K_{S}^{0} K^{+} \pi^{-}$channel, since different selections are used. The effects mentioned above probably conspire to make up the remaining discrepancy, although no obvious candidate exists.

The other measurements of $\mathcal{B}\left(B \rightarrow \eta_{c} K\right)$ include the result from CLEO (2001) [50]

$$
\begin{aligned}
\mathcal{B}\left(B^{0} \rightarrow \eta_{c} K^{0}\right) & =\left(1.09_{-0.42}^{+0.55} \pm 0.12 \pm 0.31\right) \times 10^{-3} \\
\mathcal{B}\left(B^{+} \rightarrow \eta_{c} K^{+}\right) & =\left(0.69_{-0.21}^{+0.26} \pm 0.08 \pm 0.20\right) \times 10^{-3},
\end{aligned}
$$

and Belle (2002) [23]

$$
\begin{aligned}
\mathcal{B}\left(B^{0} \rightarrow \eta_{c} K^{0}\right) & =\left(1.23 \pm 0.23_{-0.16}^{+0.12} \pm 0.38\right) \times 10^{-3} \\
\mathcal{B}\left(B^{+} \rightarrow \eta_{c} K^{+}\right) & =\left(1.25 \pm 0.14_{-0.12}^{+0.10} \pm 0.38\right) \times 10^{-3} .
\end{aligned}
$$

The size of the samples used in the CLEO and Belle analyses were approximately $11 \%$ and $37 \%$ of the sample size used in this analysis. 
The branching fraction results obtained here agree rather well with the recent results from Belle. The observed change with respect to the previous BABAR measurement (5.21) is consistent with the statistical errors on the measurements.

It is clear that the $\mathcal{B}\left(B \rightarrow \eta_{c} K\right)$ results presented here are of the same order as the world average measurements of $\mathcal{B}(B \rightarrow J / \Psi K)[5]$,

$$
\begin{aligned}
\mathcal{B}\left(B^{0} \rightarrow J / \Psi K^{0}\right) & =(0.87 \pm 0.05) \times 10^{-3} \\
\mathcal{B}\left(B^{+} \rightarrow J / \Psi K^{+}\right) & =(1.01 \pm 0.05) \times 10^{-3} .
\end{aligned}
$$

as predicted (see Section 1.6). The ratios $R_{K}=\mathcal{B}\left(B \rightarrow \eta_{c} K\right) / \mathcal{B}(B \rightarrow J / \Psi K)$ are given by

$$
\begin{aligned}
& R_{K}^{0}=1.43 \pm 0.54 \\
& R_{K}^{+}=1.22 \pm 0.42,
\end{aligned}
$$

where the error is dominated by the $\mathcal{B}\left(\eta_{c} \rightarrow K \bar{K} \pi\right)$ errors in the $\mathcal{B}\left(B \rightarrow \eta_{c} K\right)$ measurements. Predictions for $R_{K}$ range lie in the approximate range 0.9 to 1.6. (see Section 1.6).

Thus these results support the general methods used in the $R_{K}$ predictions (including effective Hamiltonians of $B$ weak decays and factorization), while they do not favour any individual prediction.

As well as providing the branching fraction measurements, this analysis also presents interesting information on the $\eta_{c}$ meson and its $K \bar{K} \pi$ decay. Hints of $K^{*}(1430) \bar{K}$ intermediate resonances in the $\eta_{c} \rightarrow K \bar{K} \pi$ decays are seen in the $\eta_{c} \rightarrow K \bar{K} \pi$ Dalitz plots (Figure 5.20); however, an analysis of the individual contributions is not attempted here. A crude estimate of the $\eta_{c}$ width is obtained from the fits to the invariant $K \bar{K} \pi$ mass spectra (Figure 5.19), yielding an estimate of $\Gamma\left(\eta_{c}\right)=42.7 \pm 7.7 \mathrm{MeV} / c^{2}\left(\Gamma\left(\eta_{c}\right)=24.3 \pm 14.2 \mathrm{MeV} / c^{2}\right)$ in the $\eta_{c} \rightarrow K_{S}^{0} K^{+} \pi^{-}$ $\left(\eta_{c} \rightarrow K^{+} K^{-} \pi^{0}\right)$ channel. These estimations (subject to unstudied systematic ef- 
fects) are more consistent with the recent measurements of the $\eta_{c}$ width (see Section 1.6.2) than with the world average. 


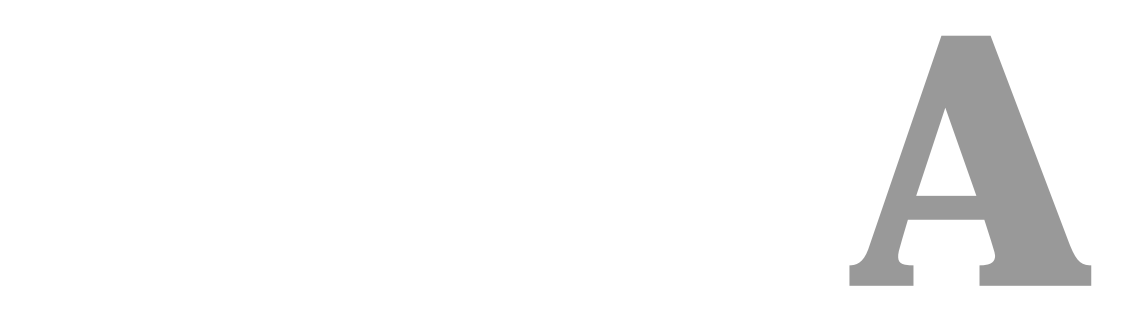

\section{Isospin Decomposition of} $\eta_{c} \rightarrow K \bar{K} \pi$ Final States

The $\eta_{c}$ meson has isospin quantum numbers $I=0$ and $I_{3}=0$. For the $\eta_{c} \rightarrow K \bar{K} \pi$ decay to conserve isospin, the $K \bar{K} \pi$ state must also have a $I=0, I_{3}=0$ configuration, denoted here as $|K \bar{K} \pi ; 00\rangle$. The isospin decomposition of the $|K \bar{K} \pi ; 00\rangle$ state is therefore prescribed by Clebsch-Gordon coefficients when combing two angular momentum states. The two states may be chosen as the $K \bar{K}$ system and the pion, each with total isospin $I=1$ so that the $K \bar{K} \pi$ system has $I=0$. The 
decomposition is given by

$$
\begin{aligned}
& |K \bar{K} \pi ; 00\rangle= \\
& \quad\left|I^{K \bar{K}}=1, I^{\pi}=1 ; I=0, I_{3}=0\right\rangle=\sum_{I_{3}^{K} \bar{K}_{,} I_{3}^{\pi}} C\left(I, I_{3}\right)\left|I^{K \bar{K}}, I^{\pi} ; I_{3}^{K \bar{K}} I_{3}^{\pi}\right\rangle .
\end{aligned}
$$

Using the tabulated values of the Clebsch-Gordon coefficients $C\left(I, I_{3}\right)$ for $(1 \times 1)$ [5], this becomes

$$
\begin{aligned}
& |K \bar{K} \pi ; 00\rangle= \\
& \quad \frac{1}{\sqrt{3}}\left|I_{3}^{K \bar{K}}=+1, I_{3}^{\pi}=-1\right\rangle-\frac{1}{\sqrt{3}}\left|I_{3}^{K \bar{K}}=0, I_{3}^{\pi}=0\right\rangle+\frac{1}{\sqrt{3}}\left|I_{3}^{K \bar{K}}=-1, I_{3}^{\pi}=+1\right\rangle,
\end{aligned}
$$

where the identical total isospin quantum numbers in each term have been omitted. The terms of the right hand side of (A.2) correspond to $\left|K \bar{K} \pi^{-}\right\rangle,\left|K \bar{K} \pi^{0}\right\rangle,\left|K \bar{K} \pi^{+}\right\rangle$ with $I_{3}^{K \bar{K}}=+1,0,-1$ respectively. The $K \bar{K}$ system in each term can be further decomposed using the Clebsch-Gordon coefficients for $(1 / 2 \times 1 / 2)$. For the first and third terms this simply gives $\left|\bar{K}^{0} K^{+} \pi^{-}\right\rangle$and $\left|K^{0} K^{-} \pi^{+}\right\rangle$respectively, while in the decomposition of second term only $K \bar{K}$ states with zero charge are relevant to the $\eta_{c} \rightarrow K \bar{K} \pi$ decay, which leads to

$$
\begin{aligned}
|K \bar{K} \pi ; 00\rangle & =\frac{1}{\sqrt{3}}\left|\bar{K}^{0} K^{+} \pi^{-}\right\rangle \\
& -\frac{1}{\sqrt{12}}\left|K^{+} K^{-} \pi^{0}\right\rangle \\
& -\frac{1}{\sqrt{12}}\left|K^{-} K^{+} \pi^{0}\right\rangle \\
& -\frac{1}{\sqrt{12}}\left|K^{0} \overline{K^{0}} \pi^{0}\right\rangle \\
& -\frac{1}{\sqrt{12}}\left|\bar{K}^{0} K^{0} \pi^{0}\right\rangle \\
& +\frac{1}{\sqrt{3}}\left|K^{0} K^{-} \pi^{+}\right\rangle .
\end{aligned}
$$

For experimental situations, where charge conjugates of detected final states are often analysed together, it is useful to group the charge conjugate terms. This 
means that a $K \bar{K} \pi$ state with $I=0$ and $I_{3}=0$, (such as that produced in an $\eta_{c}$ decay) contains contributions from $K^{0} K^{-} \pi^{+}, K^{+} K^{-} \pi^{0}$ and $K^{0} \bar{K}^{0} \pi^{0}$ in the ratios $\frac{2}{3}: \frac{1}{6}: \frac{1}{6}$. 


\section{Curve Fitting Using Orthogonal Polynomials}

The least squares [33] method of fitting a polynomial $f(x)=c_{0}+c_{1} x+\ldots c_{n} x^{n}$ to a set of points $\left(x_{i}, y_{i}\right)$ is to minimise the chi-squared quantity

$$
\chi^{2}=\sum_{i} \frac{\left(c_{0}+c_{1} x_{i}+\ldots c_{n} x_{i}^{n}-y_{i}\right)^{2}}{\sigma_{i}^{2}},
$$


where $\sigma_{i}$ are the errors on the $y_{i}$ values. If the $\sigma_{i}$ are uncorrelated, the chi-squared minimisation yields a set of equations for the coefficients $c_{i}$ which can be expressed

$$
\left(\begin{array}{c}
c_{0} \\
c_{1} \\
\vdots \\
c_{n}
\end{array}\right)=\left(\begin{array}{cccc}
\sum_{i} \frac{1}{\sigma_{i}^{2}} & \sum_{i} \frac{x_{i}}{\sigma_{i}^{2}} & \ldots & \sum_{i} \frac{x_{i}^{n}}{\sigma_{i}^{2}} \\
\sum_{i} \frac{x_{i}}{\sigma_{i}^{2}} & \sum_{i} \frac{x_{i}^{2}}{\sigma_{i}^{2}} & \ldots & \sum_{i} \frac{x_{i}^{n+1}}{\sigma_{i}^{2}} \\
\vdots & & & \\
\sum_{i} \frac{x_{i}^{n}}{\sigma_{i}^{2}} & \sum_{i} \frac{x_{i}^{n+1}}{\sigma_{i}^{2}} & \ldots & \sum_{i} \frac{x_{i}^{2 n}}{\sigma_{i}^{2}}
\end{array}\right)^{-1} \quad\left(\begin{array}{c}
\sum_{i} \frac{y_{i}}{\sigma_{i}^{2}} \\
\sum_{i} \frac{x_{i} y_{i}}{\sigma_{i}^{2}} \\
\vdots \\
\sum_{i} \frac{x_{i}^{n} y_{i}}{\sigma_{i}^{2}}
\end{array}\right) .
$$

A polynomial with more easily and cleanly calculable coefficients can be defined $f(x)=c_{0}^{\prime} P_{0}(x)+c_{1}^{\prime} P_{1}(x)+\ldots c_{n}^{\prime} P_{n}(x)$ where

$$
P_{n}(x)=a_{0 n}+a_{1 n} x+\ldots a_{n n} x^{n}
$$

with coefficients $a_{m n}$ chosen by defining $a_{n n}=1$ and imposing the orthogonality condition

$$
\sum_{i} \frac{P_{n}\left(x_{i}\right) P_{m}\left(x_{i}\right)}{\sigma_{i}^{2}}=0 \quad \text { unless } n=m,
$$

Hence $P_{0}(x)=a_{00} x^{0}=1$ and the other $P_{n}(x)$ are determined iteratively, redefining

$$
P_{n}(x)=b_{0 n} P_{0}(x)+b_{1 n} P_{1}(x)+\ldots x^{n},
$$

and using the orthogonality relation to obtain

$$
b_{m n}=-\frac{\sum_{i} \frac{x_{i}^{m} P_{n}\left(x_{i}\right)}{\sigma_{i}^{2}}}{\sum_{i} \frac{P_{n}^{2}\left(x_{i}\right)}{\sigma_{i}^{2}}},
$$

Once the $P_{n}(x)$ are determined for the range of values $x_{i}$ over which the fit is to be done, then the calculation of the $c_{l}^{\prime}$ coefficients from the least squares best fit estimate is much simpler since the matrix involved in (B.2) is diagonal (due to the orthogonality of $\left.P_{n}(x)\right)$ and therefore easily invertible, yielding coefficients

$$
c_{l}^{\prime}=\frac{\sum_{i} y_{i} P_{l}\left(x_{i}\right) / \sigma_{i}^{2}}{\sum_{i} P_{l}^{2}\left(x_{i}\right) / \sigma_{i}^{2}} .
$$

The errors $\sigma\left(c_{l}^{\prime}\right)$ can be read from the covariance matrix of the $c_{l}^{\prime}$ coefficients which 
can be shown to be

$$
\mathbf{V}(\boldsymbol{c})=\left(\begin{array}{cccc}
\sum_{i} \frac{P_{0}^{2}\left(x_{i}\right)}{\sigma_{i}^{2}} & \sum_{i} \frac{P_{0}\left(x_{i}\right) P_{1}\left(x_{i}\right)}{\sigma_{i}^{2}} & \ldots & \sum_{i} \frac{P_{0}\left(x_{i}\right) P_{n}\left(x_{i}\right)}{\sigma_{i}^{2}} \\
\sum_{i} \frac{P_{1}\left(x_{i}\right) P_{0}\left(x_{i}\right)}{\sigma_{i}^{2}} & \sum_{i} \frac{P_{1}^{2}\left(x_{i}\right)}{\sigma_{i}^{2}} & \ldots & \sum_{i} \frac{P_{1}\left(x_{i}\right) P_{n}\left(x_{i}\right)}{\sigma_{i}^{2}} \\
\vdots & & & \\
\sum_{i} \frac{P_{0}\left(x_{i}\right) P_{n}\left(x_{i}\right)}{\sigma_{i}^{2}} & \sum_{i} \frac{P_{1}\left(x_{i}\right) P_{n}\left(x_{i}\right)}{\sigma_{i}^{2}} & \ldots & \sum_{i} \frac{P_{n}^{2}\left(x_{i}\right)}{\sigma_{i}^{2}}
\end{array}\right)^{-1},
$$

which due to orthogonality of the $P_{n}(x)$ has no off-diagonal elements, reducing to

$$
\mathbf{V}(\boldsymbol{c})=\left(\begin{array}{cccc}
\sum_{i} \frac{P_{0}^{2}\left(x_{i}\right)}{\sigma_{i}^{2}} & 0 & \ldots & 0 \\
0 & \sum_{i} \frac{P_{1}^{2}\left(x_{i}\right)}{\sigma_{i}^{2}} & \ldots & 0 \\
\vdots & 0 & \ldots & \sum_{i} \frac{P_{n}^{2}\left(x_{i}\right)}{\sigma_{i}^{2}}
\end{array}\right)^{-1} .
$$

Thus the coefficients obtained from the least squares fit using orthogonal polynomials are uncorrelated and their errors are given by

$$
\sigma\left(c_{l}^{\prime}\right)=\sqrt{\left(\sum_{i} \frac{P_{l}^{2}\left(x_{i}\right)}{\sigma_{i}^{2}}\right)^{-1}} .
$$




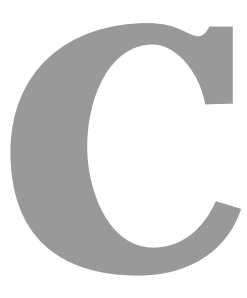

\section{Alternative Representation of}

\section{Phase Space in $\eta_{c} \rightarrow K \bar{K} \pi$ Decay}

The conventional representation of three-body decay phase space is by the Dalitz plot. Since the phase space population for $\eta_{c} \rightarrow K_{A} K_{B} \pi$ is given by

$$
d N \propto d m_{K_{A} \pi}^{2} d m_{K_{B} \pi}^{2},
$$

the phase space is illustrated by plotting $m_{K_{A} \pi}^{2}$ against $m_{K_{B} \pi}^{2}$; this is known as the Dalitz plot.

It is also possible to represent the three-body phase space by plotting the helicity 
cosine of one of the two-body subsystems against its invariant mass. For example, the helicity cosine of the $K_{A} \pi\left(K_{B} \pi\right)$ system may be chosen, defined as the cosine of the angle between the pion or kaon momentum in the $K_{A} \pi\left(K_{B} \pi\right)$ rest frame and the $K_{A} \pi\left(K_{B} \pi\right)$ momentum in the $\eta_{c}$ rest frame; the other variable plotted is then the $m_{K_{A} \pi}\left(m_{K_{B} \pi}\right)$ invariant mass. Depending on whether the pion or kaon momentum is used in the helicity cosine calculation, the cosine sign is flipped; Figure C.1 illustrates the helicity angle in the $K_{A} K_{B} \pi$ system.

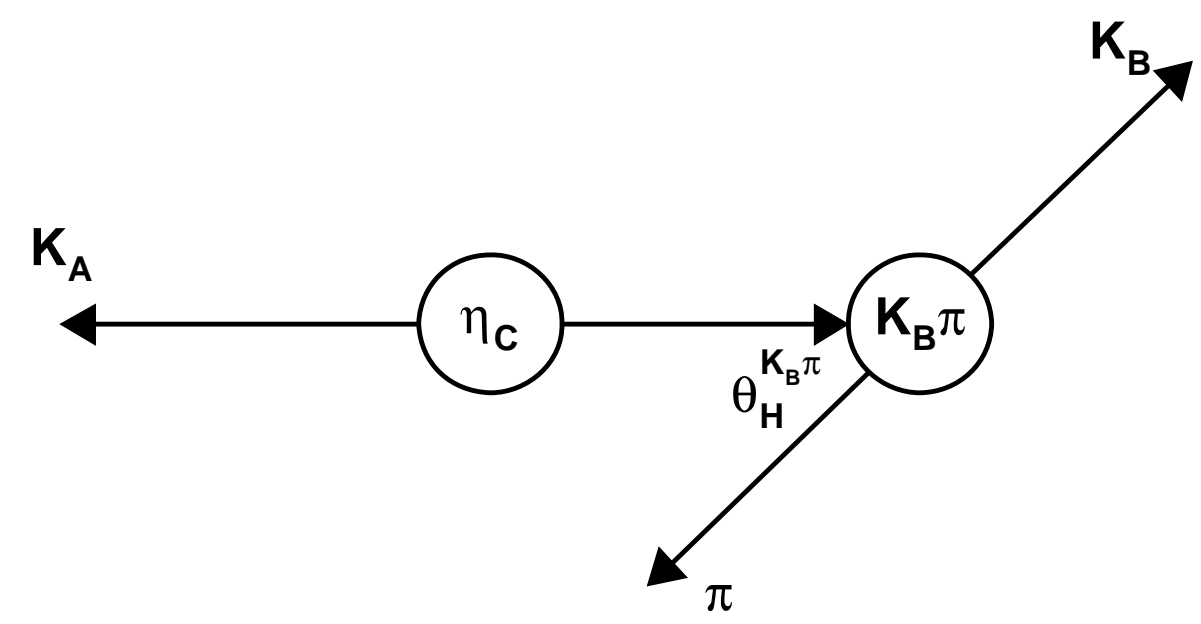

Figure C.1: Helicity angle of the $K_{B} \pi$ system, $\theta_{H}^{K_{B} \pi}$, for the three-body decay $\eta_{c} \rightarrow K_{A} K_{B} \pi$.

The transformation of the conventional Dalitz plot variables to those described above can be demonstrated in the following way. Consider the $\eta_{c} \rightarrow K_{A} K_{B} \pi$ phase space described by (C.1); the invariant mass $m_{K_{A} \pi}^{2}$ can be related to the $K_{B} \pi$ helicity cosine $\left(\cos \theta_{H}\right)^{K_{B} \pi}$ by calculating $m_{K_{A} \pi}^{2}$ in the $K_{B} \pi$ rest frame

$$
\begin{aligned}
m_{K_{A} \pi}^{2} & =\left(E_{K_{A}}+E_{\pi}\right)^{2}-\left(\mathbf{p}_{K_{A}}+\mathbf{p}_{\pi}\right)^{2} \\
& =m_{K_{A}}^{2}+m_{\pi}^{2}+2 E_{K_{A}} E_{\pi}-2 p_{K_{A}} p_{\pi} \cos \theta .
\end{aligned}
$$

The angle $\theta$, between the $K_{A}$ and $\pi$ momenta in the $K_{B} \pi$ rest frame, is the same as the helicity angle $\theta_{H}^{K_{B} \pi}$, since $p_{K_{A}}$ is antiparallel to $p_{K_{B} \pi}^{*}$ (the momentum of the 
$K_{B} \pi$ system in the $\eta_{c}$ frame). Since $E_{K_{A}}$ and $E_{\pi}$ are fixed for fixed $m_{K_{B} \pi}$, it follows that

$$
d m_{K_{A} \pi}^{2} \propto p_{K_{A}} p_{\pi} d\left(\cos \theta_{H}\right)^{K_{B} \pi} .
$$

Substituting this result into (C.1) yields a new expression for the phase space population,

$$
d N \propto p_{K_{A}} p_{\pi} d m_{K_{B} \pi}^{2} d\left(\cos \theta_{H}\right)^{K_{B} \pi} .
$$

This can be re-expressed by using the Lorentz transformation describing the boost of the $K_{A}$ momentum in the $\eta_{c}$ frame (denoted $q$, and equal to the $K_{B} \pi$ momentum in the $\eta_{c}$ frame) into the $K_{B} \pi$ frame

$$
p_{K_{A}}=\gamma\left(q+\beta E_{K_{A}}^{*}\right) .
$$

Since $\gamma=E_{K_{B} \pi}^{*} / m_{K_{B} \pi}$ and $\beta=q / E_{K_{A} \pi}^{*}$, the Lorentz transformation reduces after further steps to

$$
p_{K_{A}}=q m_{\eta_{c}} / m_{K_{B} \pi}
$$

Substituting (C.6) into (C.4) gives

$$
d N \propto q p_{\pi} d m_{K_{B} \pi} d\left(\cos \theta_{H}\right)^{K_{B} \pi} .
$$

where $q$ and $p_{\pi}$ are given by two-body decay energy-momentum conservation [5]

$$
\begin{aligned}
q & =\frac{\left[\left(m_{\eta_{c}}^{2}-\left(m_{K_{B} \pi}+m_{K_{A}}\right)^{2}\right)\left(m_{\eta_{c}}^{2}-\left(m_{K_{B} \pi}-m_{K_{A}}\right)^{2}\right)\right]^{1 / 2}}{2 m_{\eta_{c}}}, \\
p_{\pi} & =\frac{\left[\left(m_{K_{B} \pi}^{2}-\left(m_{K_{B}}+m_{\pi}\right)^{2}\right)\left(m_{K_{B} \pi}^{2}-\left(m_{K_{B}}-m_{\pi}\right)^{2}\right)\right]^{1 / 2}}{2 m_{K_{B} \pi}} .
\end{aligned}
$$

Thus the phase space can be plotted in the independent variables $\left(\cos \theta_{H}\right)^{K_{B} \pi}$ and $m_{K_{B} \pi}$. Clearly $-1<\left(\cos \theta_{H}\right)^{K_{B} \pi}<1$, while the upper and lower limits on $m_{K_{B} \pi}$ are given (from (C.7) and (C.8)) by

$$
\begin{aligned}
& \left(m_{K_{B} \pi}\right)_{\max }=m_{\eta_{c}}-m_{K_{A}}, \\
& \left(m_{K_{B} \pi}\right)_{\min }=m_{K_{B}}+m_{\pi} .
\end{aligned}
$$


It follows that the kinematically allowed region in $\left(\cos \theta_{H}\right)^{K_{B} \pi}, m_{K_{B} \pi}$ is rectangular. While the phase space event population is uniform (for non-resonant $\eta_{c} \rightarrow K_{A} K_{B} \pi$ decays) in $\left(\cos \theta_{H}\right)^{K_{B} \pi}$ at any given value of $m_{K_{B} \pi}$, it is not uniform in $m_{K_{B} \pi}$ since at all values of $\left(\cos \theta_{H}\right)^{K_{B} \pi}$,

$$
\frac{d N}{d m_{K_{B} \pi}} \propto q p_{\pi} .
$$

The transformation described above, using the $\left(K_{B} \pi\right)$ subsystem, is not the only alternative representation of the Dalitz plot; the choice of two-body subsystem, and hence the corresponding helicity cosine, can be made in three ways (ignoring the trivial sign flip of the helicity cosine). The resulting rectangular scatter plots may then be chosen as follows

$$
\begin{array}{ccl}
\left(\cos \theta_{H}\right)^{K_{A} \pi} & \text { vs. } & m_{K_{A} \pi} \\
\left(\cos \theta_{H}\right)^{K_{B} \pi} & \text { vs. } & m_{K_{B} \pi} \\
\left(\cos \theta_{H}\right)^{K_{B} K_{A}} & \text { vs. } & m_{K_{B} K_{A}} .
\end{array}
$$




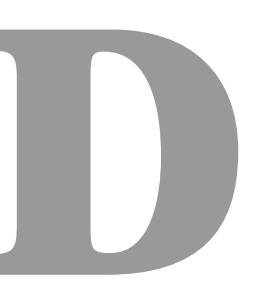

\section{Event-Shape Variables}

The 9 event-shape variables used in the Fisher discriminant to separate signal $B \rightarrow$

$\eta_{c} K$ events from continuum background (Section 4.5.4) are illustrated in Figure D.1. The variables include:

$\boldsymbol{R}_{2}$

$R_{2}$ is the ratio of the second to the zeroth Fox-Wolfram moments [60]. The FoxWolfram moments are defined by

$$
H_{l}=\sum_{i, j} \frac{\left|\boldsymbol{p}_{i}\right| \cdot\left|\boldsymbol{p}_{j}\right|}{E_{\mathrm{vis}}^{2}} P_{l}\left(\cos \theta_{i j}\right),
$$



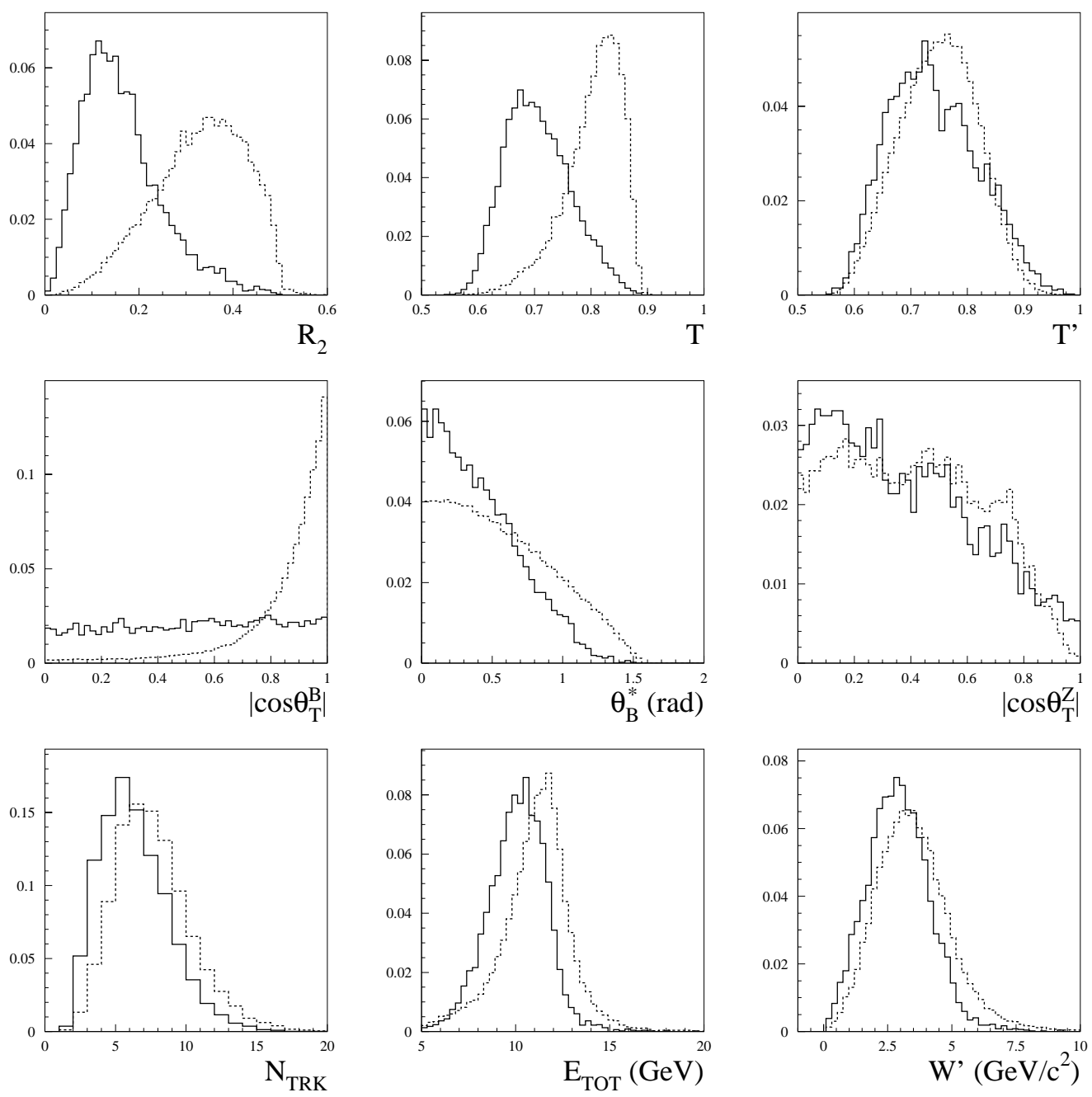

Figure D.1: Distribution of variables used in the Fisher discriminant for truth-matched $B \rightarrow \eta_{c} K, \eta_{c} \rightarrow K^{+} K^{-} \pi^{0}$ Monte Carlo (solid line) and off resonance data (dashed line). The distributions are normalised to unit area.

where $P_{l}$ are the Legendre polynomials, $\boldsymbol{p}_{i, j}$ are the particle momenta, $\theta_{i j}$ is the opening angle between particles $i$ and $j$, and $E_{\mathrm{vis}}$ is the total visible energy of the event. Both charged and neutral particles were used in the $R_{2}$ calculation.

$T$ is the event thrust. The event thrust axis $\hat{T}$ is the direction that maximises the 
sum of the longitudinal momentum of the charged and neutral particles in the event. The event thrust is given by

$$
T=\frac{\sum_{i}\left|\hat{T} \cdot \mathbf{p}_{i}\right|}{\sum_{i}\left|\mathbf{p}_{i}\right|}
$$

Jet-like events in which all the momenta lie in a similar direction have $T \sim 1$, while more spherical events have $T \sim 0.5$

\section{$T^{\prime}$}

$T^{\prime}$ is the thrust of the particles in the event excluding those from $B$ candidate, and provides a similar, although less effective, separation of signal and background candidates as the normal thrust variable $T$.

\section{$\left|\cos \theta_{T}^{B}\right|$}

$\left|\cos \theta_{T}^{B}\right|$ is the angle between the thrust axis of the particles from the $B$ candidate and the thrust axis of the rest of the event. If the event consists of two $B$ decays, the two thrust axes should be uncorrelated and $\left|\cos \theta_{T}^{B}\right|$ should be uniformly distributed. More directional events are indicated by a distribution peaked at $\left|\cos \theta_{T}^{B}\right| \sim 1$.

$\theta_{B}^{*}$

$\theta_{B}^{*}$ is the polar angle of the $B$ candidate momentum in the $\Upsilon(4 S)$ frame. The spherical nature of $B \bar{B}$ events means that the reconstruction efficiency of reconstruction of $B$ mesons is lower for $B$ momenta aligned with the $z$-axis, due to detector acceptance. Fake $B$ candidates from jet-like events are more likely to populate detector regions near the acceptance limit (in Figure D.1, the polar angle is offset by $-\pi / 2$ ).

$\left|\cos \theta_{T}^{Z}\right|$

$\left|\cos \theta_{T}^{Z}\right|$ is the polar angle of the thrust axis, and provides a similar discrimination to $\theta_{B}^{*}$. Only charged particles were used to determine $\left|\cos \theta_{T}^{Z}\right|$. 


\section{$N_{T R K}$}

$N_{T R K}$ is the total number of reconstructed tracks in the event, excluding those used to reconstruct the $B$ meson. Jet-like events will in general contain more tracks than $B \bar{B}$ events.

\section{$E_{T O T}$}

$E_{T O T}$ is the total energy of all charged and neutral particles in the event. Detector acceptance results in a higher average $E_{T O T}$ for continuum events than for $B \bar{B}$ events.

$W^{\prime}$

$W^{\prime}$ is the summed invariant mass of all the particles in the event, excluding those used to reconstruct the $B$ meson. $W^{\prime}$ provides a similar discrimination to $E_{T O T}$. The particle from the $B$ were excluded so as to remove the correlation of the variable with the mass of the $B$, which might bias the $M_{E S}$ distribution when the Fisher selection is applied. 


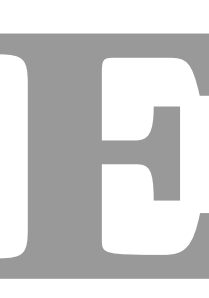

\section{Fast Monte Carlo (PravdaMC)}

The simulation of data at BABAR is a lengthy and technically complicated procedure, in which background and month-by-month detector conditions are folded into each generated simulated event. Production of the Monte Carlo samples used for the many $B A B A R$ physics analyses is usually done by a central task force - the Simulation Working Group.

An overview of the Monte Carlo production is as follows. The four-momenta of the particles produced in a particular physics process following the $e^{+} e^{-}$interaction are created by an event generator. $B \bar{B}$ events are generated with the package EvtGen 
[61], while hadronic continuum events are generated with JETSET [62]. Particle transport through the BABAR detector is simulated using the GEANT4 ${ }^{1}$ package, which includes the effect of the BABAR magnetic field, particle energy loss, multiple scattering, and particle decay. The detector hits produced by this step are then transformed into simulated digitised signals. Real background events from trigger readouts taken at random are mixed with the simulated events. The normal BABAR reconstruction software then builds the basic event quantities in an identical fashion to real event data reconstruction.

A fast simulation package (PravdaMC) has been developed at BABAR which is less elaborate than the process described above, and is able to generate and reconstruct events much more quickly. PravdaMC is a convenient tool to study the topology of processes and the shapes of signal and background distributions, without having to resort to the full Monte Carlo production described above. PravdaMC uses the same event generators as the full Monte Carlo but not the GEANT4-based particle transport. Instead, the simulation of detector tracks is performed by the TrackErr [3] package. This package assumes a uniform magnetic field and therefore creates helical charged particle trajectories, which are then smeared to take into account detector response. Particle energy loss and particle identification information are not simulated in PravdaMc; therefore the efficiency of particle identification selectors (see Section 4.5.5) measured with data control samples controls the number of particles surviving particle identification-based selection. Final state charged kaons or pions in PravdaMC do not decay; they are effectively simulated as infinitely stable particles.

PravdaMC was used in the branching fraction analysis of $B^{0} \rightarrow \eta_{c} K_{S}^{0}$ and $B^{+} \rightarrow$ $\eta_{c} K^{+}$described in Chapters 4 and 5 . The average efficiency of tracks belonging to $B \rightarrow \eta_{c} K$ candidates, measured by the ratio of the number of reconstructed, truthmatched (see Section 4.2.2) tracks to the number of generated 'tracks' was found to

\footnotetext{
${ }^{1} \mathrm{An}$ 'object-orientated' version of GEANT [63], written in $\mathrm{C}++$.
} 
be 5\% (per track) higher in PravdaMC than in full Monte Carlo [64]. This is not so surprising considering the naive nature of simulation described above. Since 6 tracks are required to reconstruct a $B$ meson in the $B^{0} \rightarrow \eta_{c} K_{S}^{0}, \eta_{c} \rightarrow K_{S}^{0} K^{+} \pi^{-}$channels, the SP4-PravdaMC track efficiency difference results in a large discrepancy in the final $B$ candidate selection efficiency. A facility exists in PravdaMC for randomly removing tracks from each event which was employed to suppress the track efficiency by $5 \%$ per track. However even after this correction was applied, the final $B$ candidate selection efficiency in SP4 Monte Carlo was still only two-thirds that measured with PravdaMC. The same level of disagreement was observed when measuring the $B$ preselection efficiency (including the 5\% track suppression) by the ratio of the number of truthmatched $B$ candidates to generated events. This remaining discrepancy is most likely due to poorer track momentum resolution in SP4 Monte Carlo, which results in long tails in the reconstructed mass distributions for genuine $K_{S}^{0}$ and $\eta_{c}$ decays. Long tails were indeed observed in the truth-matched $K_{S}^{0}$ mass distributions in SP4 $B^{0} \rightarrow \eta_{c} K_{S}^{0}, \eta_{c} \rightarrow K_{S}^{0} K^{+} \pi^{-}$Monte Carlo, which were not seen in PravdaMC [65]. Thus an appreciable number of $B$ decays do not survive the loose $K_{S}^{0}$ mass selection in the preselection in SP4. The fact that two $K_{S}^{0}$ are present in each $B^{0} \rightarrow \eta_{c} K_{S}^{0}, \eta_{c} \rightarrow K_{S}^{0} K^{+} \pi^{-}$event, along with similar resolution effects in the $\eta_{c}$ mass and $\Delta E$ distributions are likely to account for the final difference in candidate selection efficiency in SP4 and PravdaMC.

Apart from the resolution effects which affect the tails of the mass distributions, the selection variable distributions in PravdaMC and SP4 agree reasonably well. Figure E.1 demonstrates the comparison of some of the quantities (described in Chapter 4) used for selection in the $B \rightarrow \eta_{c} K$ branching fraction analysis. 

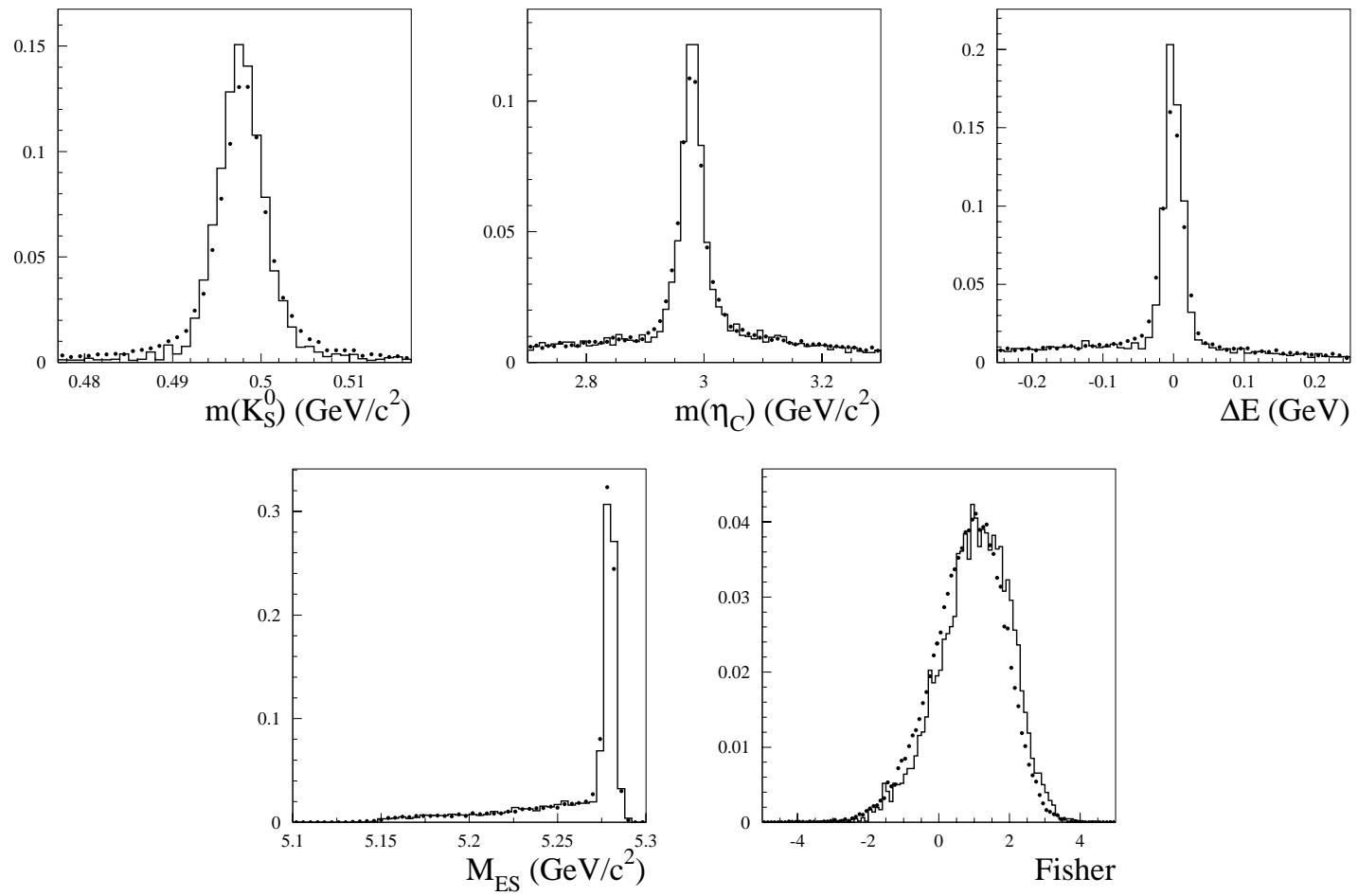

Figure E.1: Comparison of selection variables for PravdaMC (points) and SP4 Monte Carlo (line). The variables are described in Chapter 4. These variable distributions were produced by S. Ricciardi (Charmonium Working Group). 


\section{References}

[1] A. D. Sakharov, ZhETF Pis. Red. 5, 32 (1967).

[2] J. H. Christenson, J. W. Cronin, V. L. Fitch, and R. Turlay, Phys. Rev. Lett. 13, 138 (1964).

[3] P. F. Harrison and H. R. Quinn, The BABAR physics book: Physics at an asymmetric $B$ factory, (1998).

[4] Y. Nir and H. R. Quinn, Ann. Rev. Nucl. Part. Sci. 42, 211 (1992).

[5] K. Hagiwara et al., Phys. Rev. D66, 010001+ (2002).

[6] D. Perkins, Introduction to High Energy Physics, Fourth ed. (Cambridge University Press, Cambridge, UK, 2000).

[7] BABAR collaboration, B. Aubert et al., Phys. Rev. D66, 032003 (2002).

[8] BABAR collaboration, B. Aubert et al., Phys. Rev. Lett. 89, 201802 (2002).

[9] G. Buchalla, Heavy Quark Theory, Proceedings of the Fifty-Fifth Scottish Universities Summer School in Physics, St. Andrews (Scottish Universities Summer School in Physics \& Institute of Physics Publishing, Bristol, UK, 2002).

[10] N. G. Deshpande and J. Trampetic, Phys. Lett. B339, 270 (1994). 
[11] M. R. Ahmady and R. R. Mendel, Z. Phys. C65, 263 (1995).

[12] M. Gourdin, Y. Y. Keum, and X.-Y. Pham, Phys. Rev. D52, 1597 (1995).

[13] P. Colangelo, C. A. Dominguez, and N. Paver, Phys. Lett. B352, 134 (1995).

[14] D. S. Hwang and G.-H. Kim, Z. Phys. C76, 107 (1997).

[15] T. Himel et al., Phys. Rev. Lett. 45, 1146 (1980).

[16] R. Partridge et al., Phys. Rev. Lett. 45, 1150 (1980).

[17] W. Kwong, J. L. Rosner, and C. Quigg, Ann. Rev. Nucl. Part. Sci. 37, 325 (1987).

[18] F. E. Close, An Introduction to Quarks and Partons (Academic Press, London UK, 1979).

[19] W. Kwong et al., Phys. Rev. D37, 3210 (1988).

[20] B. Meyer, (2002), SLAC-BABAR-ANALYSIS-DOCUMENT-261.

[21] CLEO collaboration, G. Brandenburg et al., Phys. Rev. Lett. 85, 3095 (2000).

[22] S. N. Gupta, J. M. Johnson, and W. W. Repko, Phys. Rev. D54, 2075 (1996).

[23] Belle collaboration, F. Fang et al., Phys. Rev. Lett. 90, 071801 (2003).

[24] DM2 collaboration, D. Bisello et al., Nucl. Phys. B350, 1 (1991).

[25] D. Perkins, Introduction to High Energy Physics, Third ed. (Addison-Wesley, Wokingham UK, 1987).

[26] BABAR collaboration, B. Aubert et al., Nucl. Instrum. Meth. A479, 1 (2002).

[27] D. N. Brown, A. Charles, E, and D. A. Roberts, The BABAR track fitting algorithm, 2000, Proceedings of CHEP 2000, Padova, Italy. 
[28] R. Bernet et al., (2001), SLAC-BABAR-NOTE-363.

[29] J. A. Nash, (2002), SLAC-BABAR-NOTE-552.

[30] J. M. Bauer, (2001), SLAC-BABAR-NOTE-357.

[31] CLEO collaboration, Y. Kubota et al., Nucl. Instrum. Meth. A320, 66 (1992).

[32] A. Bukin and H. Marsiske, (1996), SLAC-BABAR-NOTE-339.

[33] R. J. Barlow, A Guide to the Use of Statistical Methods in the Physical Sciences (Wiley, UK, Chichester, 1989).

[34] R. Seitz, (1996), SLAC-BABAR-NOTE-294.

[35] Study performed by R. J. Barlow and presented to the BABAR calorimeter group, http: //www.slac.stanford.edu/BFR00T/www/Detector/Calorimeter/software/calib_mtg/pi031oct01rb.ps.gz.

[36] S. Menke, (2001), SLAC-BABAR-NOTE-528.

[37] BABAR collaboration, B. Aubert et al., (2002), hep-ex/0203040.

[38] CompositionTools User Guide, http: //www. slac.stanford.edu/BFR00T/www/Physics/Tools/Vertex/CompGuide/index.html.

[39] CharmUser Package and CompositionTools, http://www.slac.stanford.edu/BFROOT/www/Physics/Analysis/AWG/Charmonium/CharmUser/CharmCT.html.

[40] F. Martinez-Vidal and M. Carpinelli, (2001), SLAC-BABAR-ANALYSISDOCUMENT-102.

[41] ARGUS collaboration, H. Albrecht et al., Phys. Lett. B241, 278 (1990). 
[42] N. Barlow, PhD thesis, University of Bristol, 2003.

[43] Study performed by W. Kozanecki, presented to the $B \rightarrow \eta_{c} K$ working group, http://www.slac.stanford.edu/ 〜witold/ETACE/MTG_8Aug02/nu_cut_stdy.ps.

[44] BABAR collaboration, B. Aubert et al., Phys. Rev. Lett. 87, 151801 (2001).

[45] BABAR collaboration, B. Aubert et al., Phys. Rev. Lett. 87, 151802 (2001).

[46] G. Cowan, Statistical Data Analysis (Oxford University Press, Oxford, UK, 1998).

[47] CLEO collaboration, D. M. Asner et al., Phys. Rev. D53, 1039 (1996).

[48] G. Manchinelli and S. M. Spanier, (2001), SLAC-BABAR-ANALYSISDOCUMENT-116.

[49] A. Drescher et al., Nucl. Inst. Meth. A237, 464 (1985).

[50] CLEO collaboration, K. W. Edwards et al., Phys. Rev. Lett. 86, 30 (2001).

[51] BABAR collaboration, B. Aubert et al., (2003), hep-ex/0304006.

[52] BABAR collaboration, B. Aubert et al., (2002), hep-ex/0207089.

[53] Proposed by W. M. Dunwoodie, http://www.slac.stanford.edu/ wmd/bbkinematics/dalitz_efficiency.note.

[54] BABAR collaboration, B. Aubert et al., Phys. Rev. D65, 032001 (2002).

[55] M. Bona et al., (2001), SLAC-BABAR-ANALYSIS-DOCUMENT-324.

[56] M. Bona et al., (2000), SLAC-BABAR-ANALYSIS-DOCUMENT-19.

[57] T. Colberg, (2001), SLAC-BABAR-ANALYSIS-DOCUMENT-196. 
[58] W. Kozanecki et al., (2002), SLAC-BABAR-ANALYSIS-DOCUMENT-336.

[59] C. Hearty, (2001), SLAC-BABAR-ANALYSIS-DOCUMENT-134.

[60] G. C. Fox and S. Wolfram, Phys. Rev. Lett. 41, 1581 (1978).

[61] A. Ryd et al., (2003), SLAC-BABAR-ANALYSIS-DOCUMENT-522.

[62] T. Sjostrand, Comput. Phys. Commun. 82, 74 (1994).

[63] CERN, GEANT, detector description and simulation tool, 1994, Program library long writeup W5013.

[64] Study performed by the author and presented to the Charmonium Working Group, http://www.slac.stanford.edu/cgi-bin/lwgate/ETA-C/archives/eta-c.200207/Author/article-34.html.

[65] Study performed by the author and presented to the Charmonium Working Group, http://www.slac.stanford.edu/cgi-bin/lwgate/ETA-C/archives/eta-c.200208/Author/article-5.html. 